\title{
The Cellular Function of USP22 and its Role in Tissue Maintenance and Tumor Formation
}

\section{Dissertation}

\author{
for the award of the degree \\ "Doctor rerum naturalium" \\ of the Georg-August-Universität Göttingen, \\ within the doctoral program \\ "Molecular Biology of Cells" \\ of the Georg-August University School of Science (GAUSS) \\ submitted by \\ Robyn Laura Kosinsky \\ from Emmerich am Rhein, Germany
}

Göttingen, 2017 


\section{Thesis supervisor}

Prof. Steven A. Johnsen, Ph.D.

\section{Thesis committee}

Prof. Steven A. Johnsen, Ph.D.

Clinic for General, Visceral and Pediatric Surgery

University Medical Center Göttingen

Göttingen, Germany

Prof. Dr. rer. nat. Holger Reichardt

Institute for Cellular and Molecular Immunology

University of Göttingen Medical School

Göttingen, Germany

Prof. Dr. med. Heidi Hahn

Institute for Human Genetics

University Medical Center Göttingen

Göttingen, Germany

Date of oral examination: 17.02.2017 


\section{Affidavit}

I hereby declare that the Ph.D. thesis entitled "The Cellular Function of USP22 and its Role in Tissue Maintenance and Tumor Formation" has been written independently and with no other sources and aids than quoted.

Göttingen, 03.01.2017

Robyn Laura Kosinsky 


\section{TABLE OF CONTENTS}

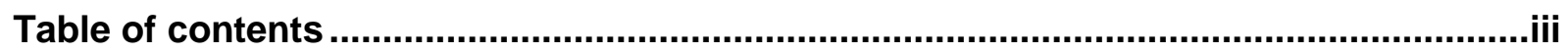

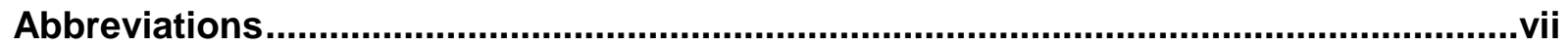

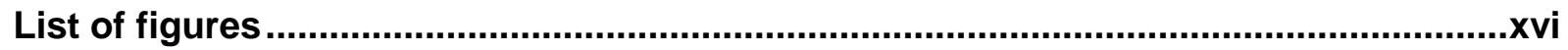

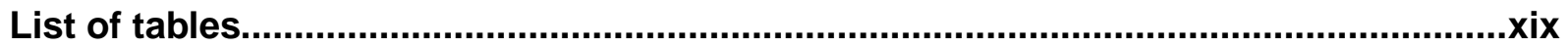

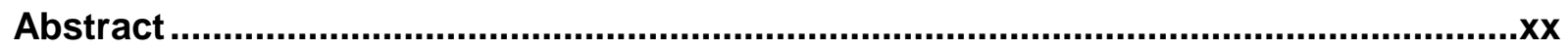

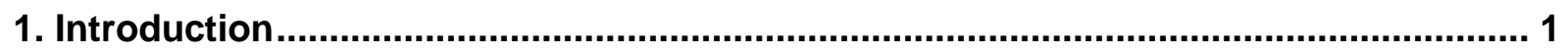

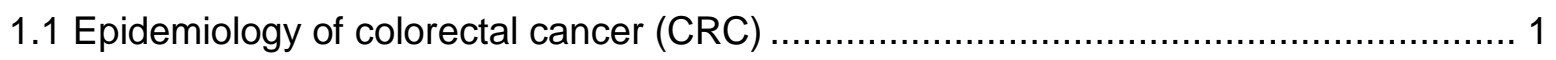

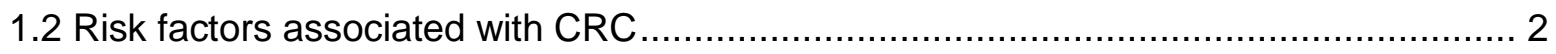

1.2.1 Diet and lifestyle .................................................................................. 2

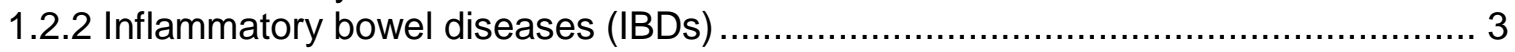

1.2.3 Genetic susceptibility ......................................................................... 4

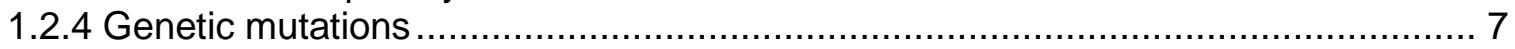

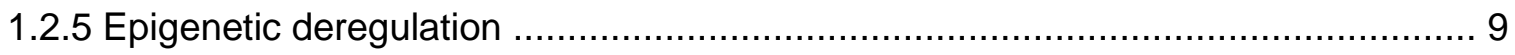

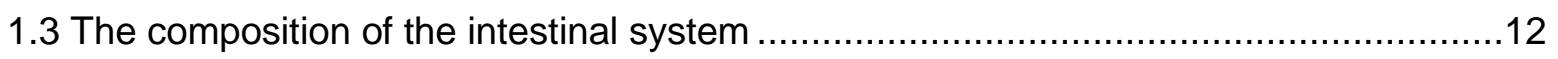

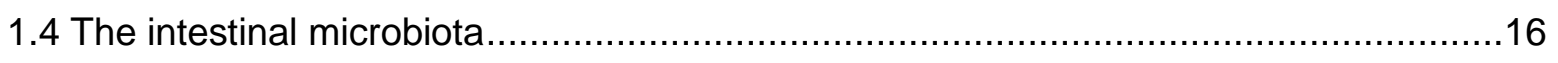

1.5 The intestinal immune defense and ulcerative colitis ............................................17

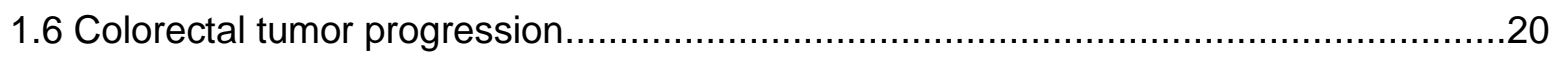

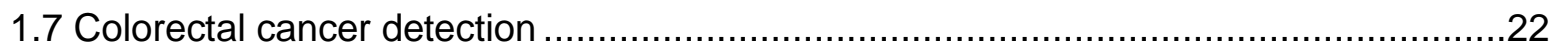

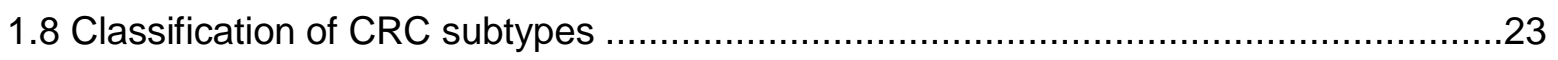

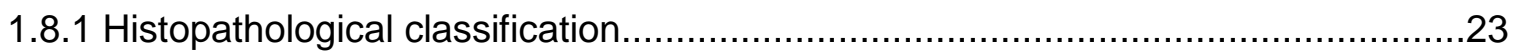

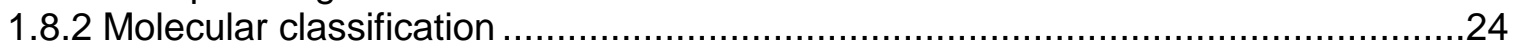

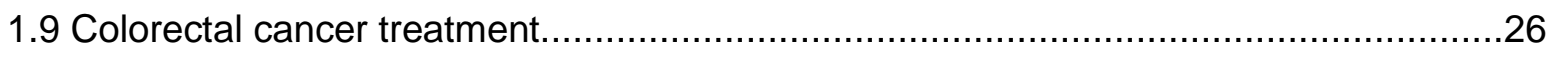

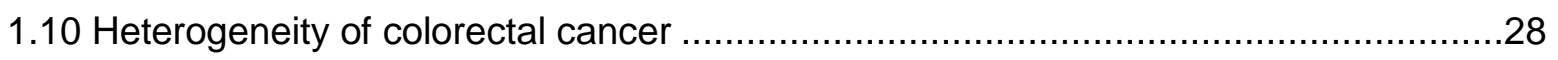

1.11 Murine models for ulcerative colitis and colorectal cancer ......................................30

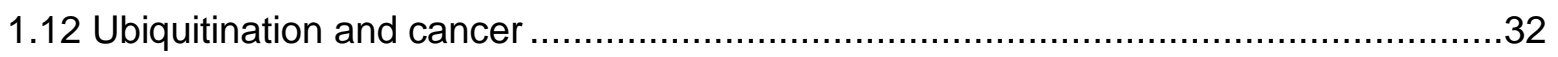




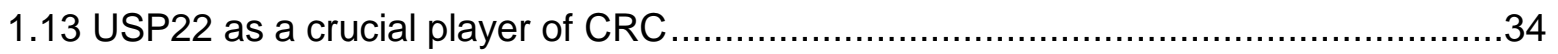

1.14 Implications of USP22 in intestinal cell differentiation (preliminary data) ..................37

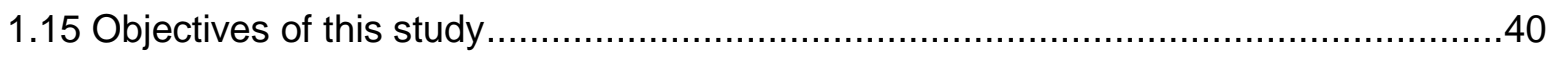

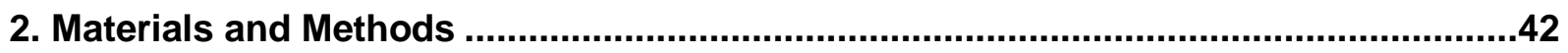

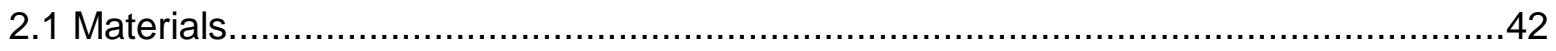

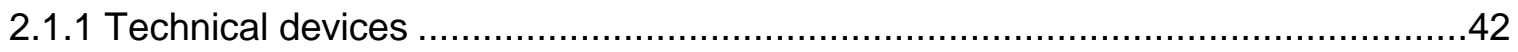

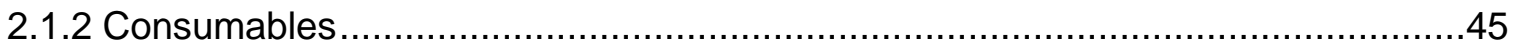

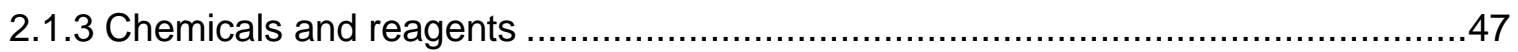

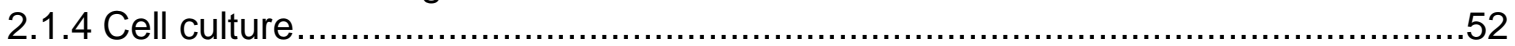

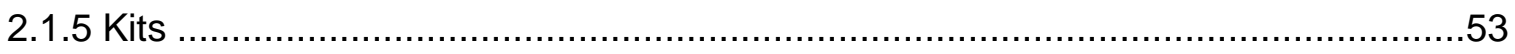

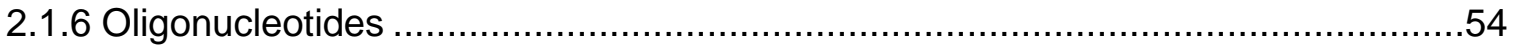

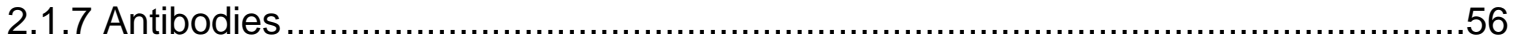

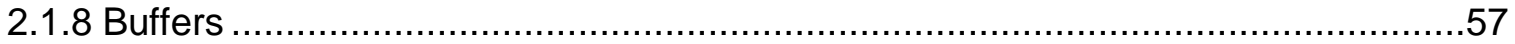

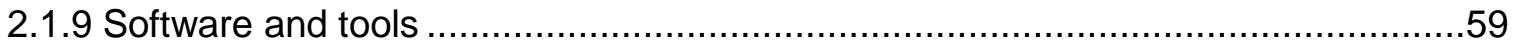

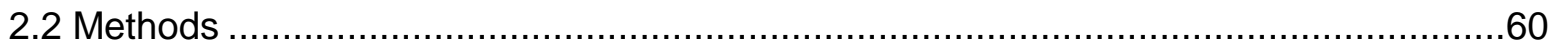

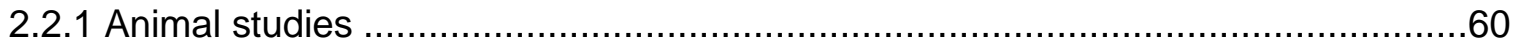

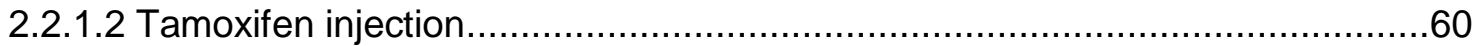

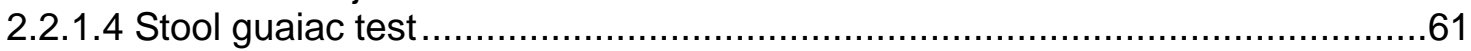

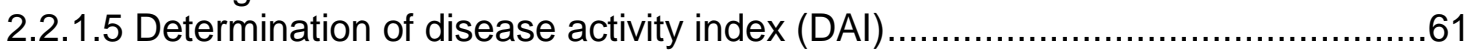

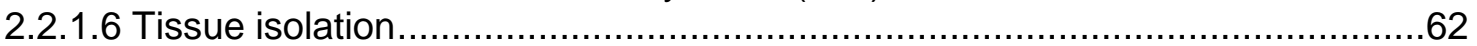

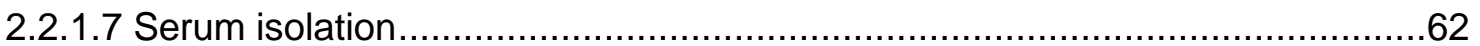

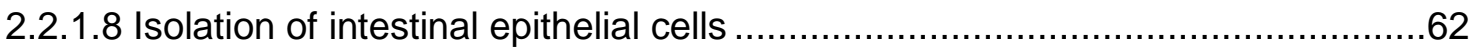

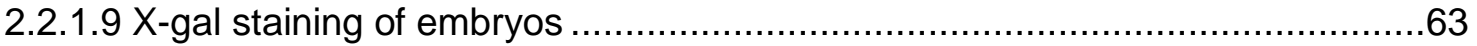

2.2.1.10 Preparation of mouse embryonic fibroblasts (MEFs) …............................63

2.2.1.11 Preparation of paraffin-embedded tissue .................................................64

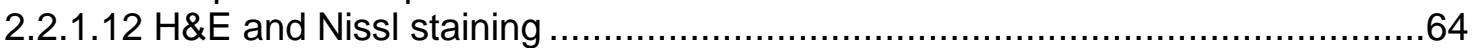

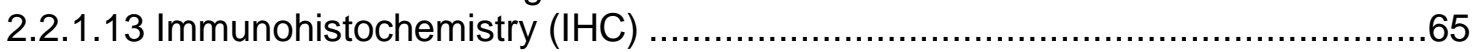

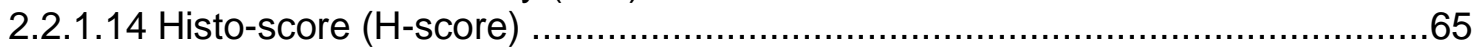

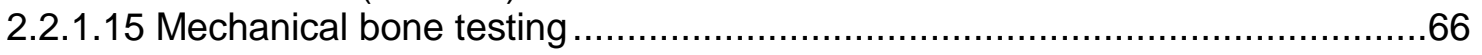

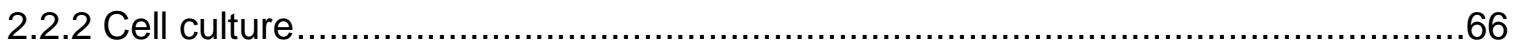

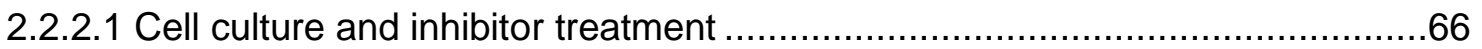

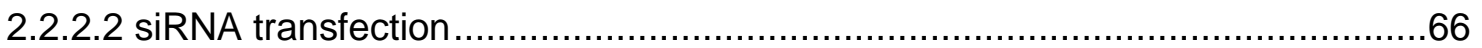

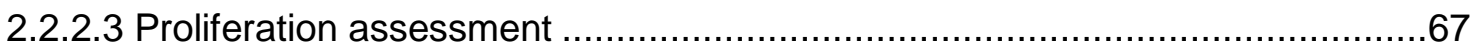

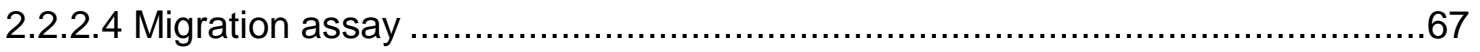

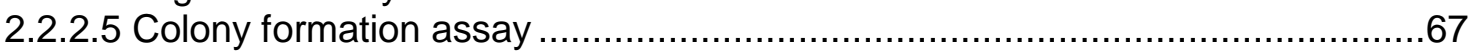

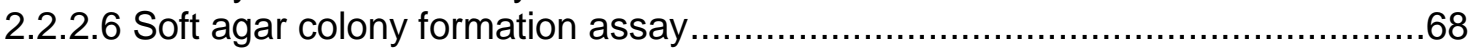

2.2.2.7 CRISPR/Cas9-mediated knockout of USP22.......................................... 68

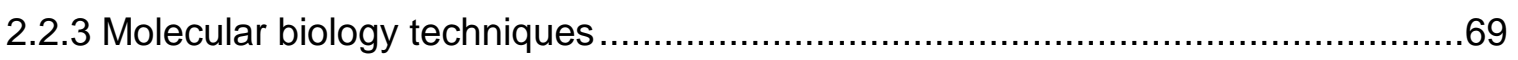

2.2.3.1 DNA extraction from tail biopsies or cells .............................................69

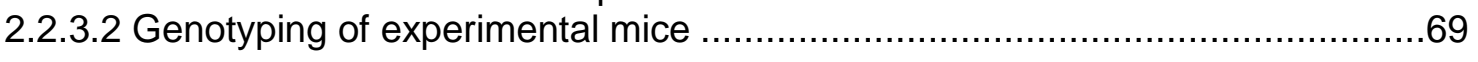




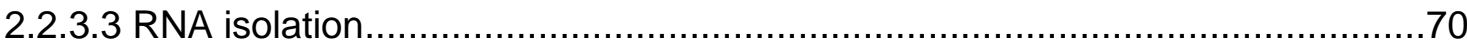

2.2.3.4 RNA gel electrophoresis ................................................................

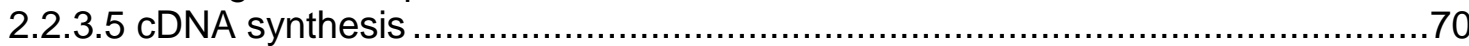

2.2.3.6 Quantitative Real-Time PCR (qPCR) .........................................................

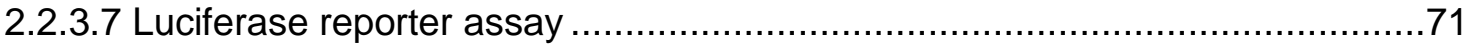

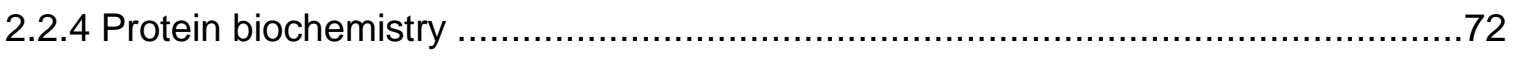

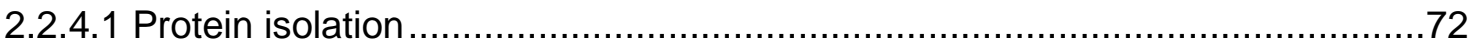

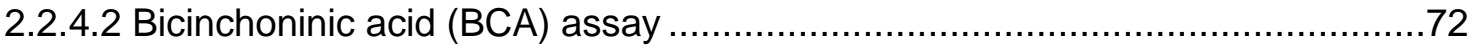

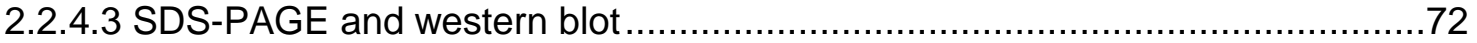

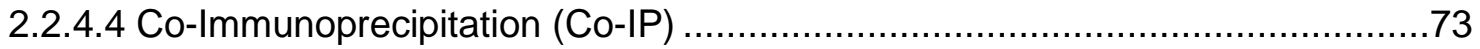

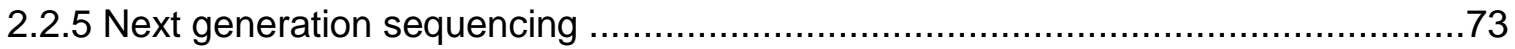

2.2.5.1 Microarray using mouse embryonic fibroblasts ..........................................73

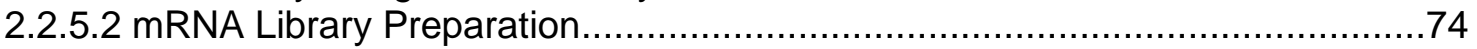

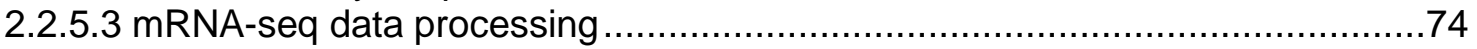

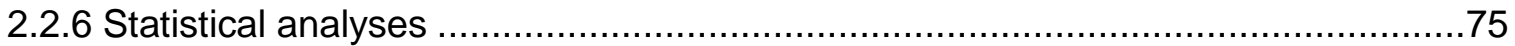

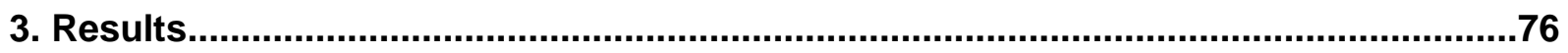

3.1 USP22 is required for proper murine development and lineage specification ..............76

3.2 The role of USP22 in colorectal tumorigenesis in vivo ............................................8

3.2.1 Inflammation-induced CRC: Intestinal Usp22 deletion prior to colitis (Tam-DSS)..91

3.2.1.1 Intestinal Usp22 loss shortens survival and increases intestinal tumor burden

3.2.1.2 Usp22 ablation is associated with inflammation and invasive carcinomas ......97

3.2.2 Inflammation-induced CRC: Colitis prior to intestinal Usp22 deletion (DSS-Tam) 106

3.2.2.1 Inducing colitis prior to Usp22 loss reduces tumor and inflammation burden 106

3.2.3 Intestinal Usp22 deletion in a model of sporadic CRC (Tam) ..........................112

3.2.3.1 Intestinal Usp22 deficiency promotes sporadic colorectal tumorigenesis ......112

3.2.4 Intestinal Usp22 deletion prior to colitis results in the worse prognosis ...............118

3.3 Intestinal Usp22 loss elevates acute colitis burden resulting in bone fragility .............122

3.4 Heterogeneous USP22 expression levels in CRC patients and cell lines .................129

3.5 USP22 regulates proliferation and differentiation processes in CRC cells ................139

3.6 USP22 regulates the stability of heat shock proteins .......................................148

3.7 Induction of synthetic lethality in USP22-deficient CRC cells ................................151

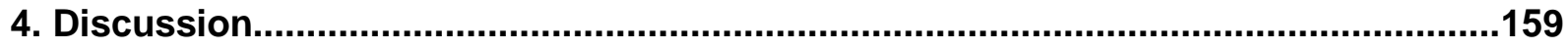


4.1 USP22 is involved in differentiation and developmental processes in vivo.

4.2 Intestinal Usp22 deletion causes mild spontaneous intestinal inflammation. 161

4.4 Intestinal Usp22 loss promotes inflammation-induced and sporadic CRC .166

4.5 Intestinal Usp22 deletion in $\mathrm{APC}^{1638 \mathrm{~N} /+}$ animals decreases life span ...... .170

4.6 The heterogeneity of USP22 expression in colorectal cancer..... 171

4.7 The interaction between USP22 and HSP90AB1 .173

4.8 USP22: oncogene or tumor suppressor? .178

4.9 USP22 as a target in colorectal cancer therapy? .180

4.10 Concluding remarks. 182

5. References . .184 Acknowledgements 


\section{AbBreviations}

\begin{tabular}{|c|c|}
\hline${ }^{\circ} \mathrm{C}$ & Degree Celsius \\
\hline$\mu$ & Micro $\left(10^{-6}\right)$ \\
\hline$A C$ & Amsterdam criteria \\
\hline ac & Acetylation \\
\hline AFAP & Attenuated familial adenomatous polyposis \\
\hline $\mathrm{APC}$ & Adenomatous Polyposis Coli \\
\hline APS & Ammonium persulfate \\
\hline ATP & Adenosine 5'-triphosphate \\
\hline ATXN7L3 & Ataxin 7 Like 3 \\
\hline BD & Behçet's disease \\
\hline BET & Bromo- and Extra-Terminal Domain \\
\hline BGP & $\beta$-Glycerolphosphate \\
\hline BIM & B-Cell Lymphoma 2 Interacting Mediator of Cell Death \\
\hline BMD & Bone mineral density \\
\hline bp & Base pair \\
\hline BRAF & B-Raf Proto-Oncogene, Serine/Threonine Kinase \\
\hline BRCA $1 / 2$ & BReast CAncer $1 / 2$ \\
\hline BRD4 & Bromodomain-Containing Protein 4 \\
\hline BSA & Bovine serum albumin \\
\hline Cat. no. & Catalog number \\
\hline CD & Crohn's disease \\
\hline CDK9 & Cyclin-Dependent Kinase 9 \\
\hline
\end{tabular}




$\begin{array}{ll}\text { CDNA } & \text { Complementary DNA } \\ \text { CDX2 } & \text { Caudal-Type Homeobox-2 } \\ \text { CGA } & \text { Chromogranin A } \\ \text { CIMP } & \text { CpG island methylator phenotype } \\ \text { CIN } & \text { Chromosomal instability } \\ \text { cm } & \text { Centimeter } \\ \text { CME } & \text { Complete mesocolic excision } \\ \text { CMS } & \text { Consensus molecular subtype } \\ \text { CO } 2 & \text { Carbon dioxide } \\ \text { CoA } & \text { Coenzyme A } \\ \text { Co-IP } & \text { Co-Immunoprecipitation } \\ \text { CP } & \text { Cortical plate } \\ \text { CpG } & \text { Cytosine-phosphatidyl-Guanine } \\ \text { CRC } & \text { Colorectal cancer } \\ \text { CTC } & \text { Computed tomographic colonography } \\ \text { CTIP2 } & \text { C2H2-Type Zinc Finger Protein } \\ \text { DAB } & \text { 3,3'-Diaminobenzidin-tetrahydrochloride } \\ \text { DAI } & \text { Disease activity index } \\ \text { DEPC } & \text { Diethylpyrocarbonate } \\ \text { DMEM } & \text { Dulbecco's Modified Eagle's Medium } \\ \text { DMSO } & \text { Dimethyl sulfoxide } \\ \text { DNA } & \text { Deoxyribonucleic acid } \\ \text { DNMT } & \text { DNA methyltransferase } \\ \text { dNTP } & \text { Deoxynucleotide triphosphate } \\ & \end{array}$




\begin{tabular}{ll} 
DOC & Deoxycholic acid \\
DSB & Double-strand break \\
dsDNA & Double-stranded DNA \\
DSS & Dextran sulfate sodium \\
DTT & Dithiotreitol \\
DUB & Deubiquitinating enzyme \\
e.g. & Exempli gratia (for example) \\
ECN & Escherichia coli strain Nissle 1917 \\
EDTA & Ethylene diamine tetraacetic acid \\
EGTA & Ethylene glycol tetraacetic acid \\
ENY2 & Enhancer of Yellow 2 Homolog \\
ERK1/2 & Extracellular-Signal-Regulated Kinase 1/2 \\
Cre ${ }^{\text {ERT2 }}$ & Cre recombinase fused to a mutant estrogen ligand-binding domain \\
EGFR & Epidermal Growth Factor Receptor \\
ESC & Embryonic stem cell \\
et al. & Et alii (and others) \\
EtOH & Ethanol \\
EZH2 & Enhancer of Zeste Homolog 2 \\
F & Forward \\
FACS & Fluorescence-activated cell sorting \\
FAP & Familial adenomatous polyposis \\
FBS & Fetal bovine serum \\
FDA & Food and Drug Administration \\
FDR & False discovery rate \\
\hline
\end{tabular}




\begin{tabular}{ll} 
FIT & Fecal immunochemical test \\
FLP & Flippase \\
FOLFIRI & Folinic acid and Irinotecan \\
FOLFOX & Folinic acid and Oxaliplatin \\
FOLFOXIRI & Folinic acid, Oxaliplatin and Irinotecan \\
FRT & FLP recombinase target \\
GALT & Gut-associated lymphoid tissue \\
GAPDH & Glyceraldehyde 3-Phosphate Dehydrogenase \\
GCN5 & General Control Of Amino-Acid Synthesis Yeast Homolog-Like 2 \\
GDP & Guanosine 5'-diphosphate \\
GIT & Gastrointestinal tract \\
GTP & Guanosine-5'-triphosphate \\
gFOBT & Guaiac-based fecal occult blood test \\
GFP & Green Fluorescent Protein \\
GO & Gene Ontology \\
GSEA & Gene set enrichment analysis \\
GSK-3 3 & Glycogen Synthase Kinase 3 Beta \\
h & Hour \\
H\&E & Hematoxylin and eosin \\
H2A & Histone 2A \\
H2B & Histone 2B \\
H2Bub1 & H2B monoubiquitination at lysine 120 \\
H3 & Histone 3 \\
H4 & Histone 4 \\
\hline
\end{tabular}




$\begin{array}{ll}\text { HDAC } & \text { Histone deacetylase } \\ \text { HNPCC } & \text { Hereditary non-polyposis colorectal cancer } \\ \text { HRP } & \text { Horseradish peroxidase } \\ \text { HSP90 } & \text { Heat Shock Protein 90 } \\ \text { HSP90AB1 } & \text { Heat Shock Protein 90 Alpha Family Class B Member 1 } \\ \text { IBD } & \text { Inflammatory bowel disease } \\ \text { i.e. } & \text { Id est (that is, namely) } \\ \text { IEC } & \text { Intestinal epithelial cells } \\ \text { IgG } & \text { Immunoglobulin G } \\ \text { IHC } & \text { Immunohistochemistry } \\ \text { IL } & \text { Interleukin } \\ \text { IPC } & \text { Intermediate precursor cells } \\ \text { ITB } & \text { Intestinal tuberculosis } \\ \text { JAB1 } & \text { Jun Activating Binding Protein 1 } \\ \text { JAMM } & \text { JAB1/MPN/MOV34-Metalloprotease } \\ \text { kDa } & \text { Kilodalton } \\ \text { KRAS } & \text { Kirsten Rat Sarcoma Viral Oncogene Homolog } \\ \text { LacZ } & \text { Lactose operon Z } \\ \text { LEF } & \text { Lymphoid Enhancer Factor } \\ \text { LGR5 } & \text { Leucine-Rich Repeat-Containing G-Protein Coupled Receptor 5 } \\ \text { loxP } & \text { Locus of Crossover in P1 } \\ \text { LYZ } & \text { Lysozyme } \\ \text { M } & \text { Molar } \\ \text { Milli (10 } 3 \text { ) }\end{array}$




\begin{tabular}{ll} 
MAC & Mucinous adenocarcinoma \\
MALT & Mucosa-associated lymphoid tissue \\
MAPK & Mitogen-Activated Protein Kinase \\
MDM2 & Mouse Double Minute 2 \\
me & Methylation \\
MEF & Mouse embryonic fibroblast \\
MEK1/2 & Mitogen-Activated Protein Kinase Kinase 1/2 \\
min & Minute \\
MJD & Machado-Joseph Domain (Josephin Domain)-Containing Protein \\
MLH1 & MutL Homologue 1 \\
MLL & Mixed-lineage leukemia \\
M-Mulv & Moloney-Murine leukemia virus \\
MMR & Mismatch repair \\
MRC & Magnetic resonance colonography \\
mRNA & Messenger RNA \\
MSH2/6 & MutS homologue 2/6 \\
MSI & Microsatellite instability \\
MSS & Microsatellite stability \\
MUC2 & Mucin 2 \\
$\mathrm{n}$ & Nano (10-9); Number of experimental samples \\
NEM & N-ethylmaleimide \\
NF-KB & Nuclear Factor Kappa B \\
NFATc2 & Nuclear Factor of Activated T cells 2 \\
NGS & Next generation sequencing \\
\hline
\end{tabular}




\begin{tabular}{ll} 
OLFM4 & Olfactomedin 4 \\
OTU & Otubain/Ovarian Tumor Domain-Containing Protein \\
P & Phosphorylation \\
padj & P-value adjusted \\
PARP & Poly(ADP-Ribose) Polymerase \\
PBS & Phosphate buffered saline \\
PCA & Principal component analysis \\
PCR & Polymerase chain reaction \\
PDK1 & Phosphoinositide-Dependent Kinase 1 \\
PFA & Paraformaldehyde \\
PGS & Protein G sepharose \\
pH & Potentia hydrogenii (potential of hydrogen) \\
PI3K & Phosphatidylinositol 3-Kinase \\
PKB & Protein kinase B (also known as AKT) \\
PMS2 & Postmeiotic Segregation Increased 2 \\
P-TEFb & Positive Transcriptional Elongation Factor b \\
PTM & Post-translational modification \\
qRT-PCR & Quantitative real-time PCR \\
R & Reverse \\
RING & Really Interesting New Gene \\
RNA & Ribonucleic acid \\
RNA Pol II & RNA polymerase II \\
RONS & Reactive oxygen and nitrogen species \\
rpm & Rounds per minute \\
\hline
\end{tabular}




$\begin{array}{ll}\text { RPMI } & \text { Roswell Park Memorial Institute Medium } \\ \text { SAGA } & \text { Spt-Ada-Gcn5 Acetyltransferase } \\ \text { SAHA } & \text { Suberoylanilidehydroxamic acid } \\ \text { SATB2 } & \text { AT-Rich Sequence-Binding Protein 2 } \\ \text { SD } & \text { Standard deviation } \\ \text { SDS } & \text { Sodium dodecyl sulfate } \\ \text { SDS-PAGE } & \text { Sodium dodecyl sulfate polyacrylamide gel electrophoresis } \\ \text { sec } & \text { Second } \\ \text { SEM } & \text { Standard error of the mean } \\ \text { sgRNA } & \text { Single guide RNA } \\ \text { SI } & \text { Small intestine } \\ \text { siControl } & \text { Non-targeting control siRNA } \\ \text { siRNA } & \text { Small interfering ribonucleic acid } \\ \text { SIRT1 } & \text { Sirtuin 1 } \\ \text { siUsp22 } & \text { siRNA directed against USP22 } \\ \text { siUsp27x } & \text { siRNA directed against USP27X } \\ \text { SOX2 } & \text { Sex-Determining Region Y-Box 2 } \\ \text { SP } & \text { Smart pool } \\ \text { SSB } & \text { Single-strand break } \\ \text { SVZ } & \text { Subventricular zone } \\ \text { TAL } & \text { Transcriptome and Genome Analysis Laboratory } \\ \text { Tam } & \text { Tamoxifen } \\ \text { Taq } & \text { Thermus aquaticus } \\ \text { TBR2 } & \text { T-Box Transcription Factor 2 } \\ \end{array}$




\begin{tabular}{ll} 
TCF & T-Cell Factor \\
TEMED & Tetramethylethylenediamine \\
TGF- $\beta$ & Transforming Growth Factor- $\beta$ \\
TME & Total mesorectal excision \\
TRF1 & Telomeric Repeat Factor 1 \\
Tris & Tris(hydroxymethyl)aminomethane \\
ub & Ubiquitination \\
UC & Ulcerative colitis \\
UCH & Ubiquitin C-terminal Hydrolase \\
UMG & University Medical Center Göttingen, Germany \\
USP22 & Ubiquitin-Specific Protease 22 \\
UV & Ultraviolet \\
V & Volt; Ventricle \\
V/v & Volume per volume \\
VEGF & Vascular Endothelial Growth Factor \\
w/v & Weight per volume \\
WAC & WW Domain-Containing Adaptor with Coiled-Coil \\
WHO & World Health Organization \\
wt & Wild type \\
\hline
\end{tabular}




\section{LIST OF FIGURES}

Figure 1: The canonical Wnt signaling pathway.

Figure 2: The interplay of $\mathrm{H} 2 \mathrm{Bub} 1$ and epigenetic regulators.

Figure 3: The human gastrointestinal tract and composition of the gastrointestinal wall.

Figure 4: Structure and cell types of the intestinal epithelium.

Figure 5: The protective structures of the intestinal system.

Figure 6: Tumor progression in colorectal cancer.

Figure 7: The heterogeneity of colorectal cancer.

Figure 8: The E1-E2-E3 process of ubiquitination and its consequences.

Figure 9: Reduced Usp22 levels lead to a differentiation shift in the small intestine.

Figure 10: Generation and phenotype of Usp22-hypomorphic mice.

Figure 11: Ubiquitous expression of Usp22 in murine embryos.

Figure 12: Reduced Usp22 levels do not alter small intestine morphology.

Figure 13: Usp22 affects cortical differentiation in the adult mouse brain.

Figure 14: H2B and H2Bub1 levels are not affected by reduced USP22 amounts in the small intestine.

Figure 15: Quality control of MEFs used for microarray analysis.

Figure 16: Gene Ontology analysis of genes affected by reduced Usp22 expression in MEFs.

Figure 17: Generation of mice with a conditional intestinal Usp22 knockout.

Figure 18: Simultaneous loss of Usp22 and APC mutation lowers life expectancy and increases the DAI in Tam-DSS mice.

Figure 19: Reduced Usp22 levels significantly elevate intestinal tumor burden.

Figure 20: Usp22 loss results in the accumulation of tumors in the proximal SI region.

Figure 21: Morphological overview of small intestines and colons.

Figure 22: Conditional intestinal loss of Usp22 does not affect small intestine morphology. 
Figure 23: The morphology of healthy colon segments is not affected by reduced Usp22 expression.

Figure 24: Invasive carcinomas are exclusively present in Usp22-- mice.

Figure 25: Mucinous presentation and elevated frequency of MALTs in Usp22-- colons.

Figure 26: High occurrence of inflammation-associated epithelial damage in Usp22 $2^{-1-}$ animals.

Figure 27: H2Bub1 levels are independent of the Usp22 status but show intratumoral variations.

Figure 28: Usp22 loss after DSS treatment does not affect the DAI but decreases survival rates.

Figure 29: Elevated tumor frequency and size in DSS-Tam APC ${ }^{1638 \mathrm{~N} /+}$, Usp22-/ mice.

Figure 30: Tumors tend to accumulate in the proximal/medial SI segment of DSS-Tam animals.

Figure 31: Usp22 ablation increases inflammation in DSS-Tam mice.

Figure 32: Usp22 loss alone results in decreased survival.

Figure 33: Usp22 loss leads to increased sporadic intestinal tumor formation.

Figure 34: Sporadic CRC lesions accumulate in the proximal and medial SI region in $\mathrm{APC}^{1638 \mathrm{~N} /+}, \mathrm{Usp}^{-1 /-}$ mice.

Figure 35: Usp22 loss causes mild spontaneous inflammation.

Figure 36: Survival curve analysis among treatment groups.

Figure 37: Comparison of tumor burden among treatment groups.

Figure 38: Acute colitis is correlated with increased DAI in Usp22 $2^{-/}$mice.

Figure 39: The anemic effect of an APC mutation is slightly aggravated by Usp22 loss.

Figure 40: Acute colitis results in decreased colon lengths in APC ${ }^{1638 \mathrm{~N} /+}$, Usp22-- animals.

Figure 41: Intestinal loss of Usp22 increases burden during acute colitis.

Figure 42: Acute colitis results in increased bone fragility in $\mathrm{APC}^{+/+}, \mathrm{Usp}_{2} 2^{-/-}$mice.

Figure 43: USP22 expression is heterogeneous in CRC patient samples.

Figure 44: Heterozygous USP22 deletions occur frequently in CRC patients and can be simulated by siRNA treatment in vitro. 
Figure 45: Knockdown of USP22 marginally affects the morphology of human CRC cell lines.

Figure 46: USP22 loss can have differential effects on proliferation of CRC cell lines.

Figure 47: USP22 and USP27X can have opposing effects on CRC cell proliferation.

Figure 48: USP22 is involved in regulation of migration potential, anchorage-independent growth and Wnt signaling pathway activity.

Figure 49: Quality control of SW480 and SW837 replicates used for mRNA-seq analysis.

Figure 50: Moderate consistency of differentially expressed genes after USP22 depletion in SW480, SW837 cells and MEFs.

Figure 51: Comparison of differentially expressed genes after USP22 depletion in SW480 and SW837 cells.

Figure 52: Gene Ontology terms enriched after USP22 loss in SW837 and SW480 cells.

Figure 53: The expression of the heat shock protein HSP90AB1 is reduced following USP22 depletion leading to increased cell sensitivity.

Figure 54: HSP90AB1 interacts with USP22 and is targeted by proteasomal degradation in the absence of USP22.

Figure 55: USP22-depleted cells are more sensitive to the HSP90 inhibitor Ganetespib.

Figure 56: Ganetespib treatment increases apoptosis levels in USP22-depleted cells.

Figure 57: Loss of USP22 elevates sensitivity of CRC cells towards JQ1.

Figure 58: CRISPR/Cas9 approach to permanently deplete USP22 in HCT116 cells.

Figure 59: Fluorescent cells indicate the presence of the GFP-containing CRISPR/Cas9 constructs targeting USP22.

Figure 60: CRISPR/Cas9-mediated USP22 knockout leads to increased proliferation in HCT116 cells.

Figure 61: Proposed model of the effect of USP22 loss on HSP90 levels. 


\section{LIST OF TABLES}

Table 1: Technical devices.

Table 2: Consumables.

Table 3: Chemicals and reagents.

Table 4: Enzymes.

Table 5: Inhibitors.

Table 6: Cell culture components.

Table 7: Cell lines.

Table 8: Kits.

Table 9: Primers used to genotype experimental mice.

Table 10: qRT-PCR primers for gene expression studies.

Table 11: siRNAs used for transient gene silencing in vitro.

Table 12: Primary antibodies used for western blot, Co-IP and IHC.

Table 13: Secondary antibodies.

Table 14: Software and tools.

Table 15: Usp22 levels visualized by X-gal staining in embryonic tissues at E15.5.

Table 16: GO terms enriched in Usp22 ${ }^{\text {lacz/lacz }}$ MEFs as revealed by GOTermFinder.

Table 17: Mouse numbers per experimental cohort used throughout the study to determine the function of USP22 in colorectal tumorigenesis.

Table 18: Sizes of the experimental mouse cohorts in which the effects of Usp22 ablation was observed during acute colitis.

Table 19: Growth characteristics upon USP22 loss do not correlate with MSI, CIN or CIMP status of CRC cell lines.

Table 20: GO terms enriched in siUsp22 SW837 cells as revealed by GOTermFinder.

Table 21: GO terms enriched in siUsp22 SW480 cells as revealed by GOTermFinder. 


\section{Abstract}

Colorectal cancer (CRC) is the most frequent malignancy of the gastrointestinal tract and therefore reflects a global health issue. Despite improved detection and treatment options the high mortality and morbidity rates of this disease emphasize the urgent need to unravel underlying mechanisms.

Over the last decade extensive investigation allowed the identification of numerous key players involved in the onset and progression of CRC. Importantly, epigenetics is an emerging research area which has already contributed significantly to understanding this disease. Generally, epigenetic mechanisms can affect the gene expression profile without altering the underlying DNA sequences. One mediator involved in these processes is the Ubiquitin-Specific Protease 22 (USP22) which is able to deubiquitinate the core histones H2A and H2B as well as other target proteins. Interestingly, the overexpression of USP22 in CRC patients was revealed by several studies and it was identified as a member of the so-called 11-gene "death-from-cancer" signature. This signature was correlated with poor prognosis and distant metastasis. However, the physiological function of USP22 in organ maintenance as well as its role in intestinal tumorigenesis remain to be elucidated.

In the current project we investigated the effect of a global reduction and intestinal deletion of Usp22 in vivo. By generating a global Usp22 hypomorphic mouse line we could overcome the embryonic lethal effect of a complete loss of this gene. Using a lacZ reporter gene expressed under the control of the endogenous Usp22 locus, we detected ubiquitous Usp22 expression during embryonic development. Accordingly, a global reduction of Usp22 expression resulted in systemic effects in adult mice, i.e. reduced body size and weight. Moreover, while the gross organ morphology was only marginally affected in these animals, cell differentiation and lineage specification were influenced in the brain and, as we have observed earlier, the small intestine. 
The involvement of Usp22 in developmental and differentiation processes was confirmed in mouse embryonic fibroblasts by microarray-based gene expression analysis.

Moreover, the function of Usp22 in tumorigenesis was explored in animals possessing an intestinal Usp22 loss and a truncation mutation in the tumor suppressor Adenomatous Polyposis Coli (APC). Surprisingly, Usp22 deletion combined with an APC mutation resulted in decreased survival rates, increased intestinal inflammation and tumor burden and importantly, led to the formation of invasive carcinomas with a mucinous phenotype. Our findings, that not only overexpression, as stated in the literature, but also downregulated USP22 expression can increase intestinal tumor burden were supported by in vitro and in silico analyses which revealed highly heterogeneous USP22 expression among CRC patients. Using global expression analyses we detected the involvement of USP22 in several cancer-related processes and identified the heat shock protein HSP90AB1 as an important USP22 target. Notably, we discovered that USP22 directly binds to the HSP90AB1 protein and prevents its proteasomal degradation. In addition, USP22 knockdown led to reduced HSP90AB1 expression levels. As a consequence USP22 knockdown cells were more sensitive towards elevated temperatures. Intriguingly, we were able to induce synthetic lethality in CRC cells with low USP22 levels by treating them with an HSP90 inhibitor or the Bromo- and Extra-Terminal (BET) domain inhibitor JQ1. In summary, in this study we significantly contributed to the current knowledge about USP22 by demonstrating its relevance in organ maintenance and intestinal tumor formation in vivo as well as by describing how CRC cells with low USP22 levels can be therapeutically targeted in vitro. 


\section{INTRODUCTION}

\subsection{Epidemiology of colorectal cancer (CRC)}

Cancer is a disease characterized by abnormal cell growth and has emerged as a major public health problem worldwide. Generally, incidence and mortality rates have been reduced due to preventive vaccines, early diagnosis and improved treatments for some cancers (Tiwari and Roy, 2012, 2012). However, most recent studies indicate that in some countries cancer has replaced cardiovascular diseases as the leading cause of death (Townsend et al., 2015). Preventive and therapeutic measures generate an extensive economic load (Burns et al., 2016) and, more importantly, cancer diagnosis means a significant burden for affected individuals and their families. This emphasizes the urgent need to further improve our understanding regarding the progression, suitable detection methods and treatment options for this group of diseases.

One example of successful research towards this goal is colorectal cancer (CRC) which is characterized by oncogenic growth inside the colon and rectum. Due to early diagnosis by colonoscopy and subsequent removal of pre-cancerous adenomas the mortality rate of CRC has decreased over the last decades (Bosetti et al., 2011; Vleugels et al., 2016). However, colorectal cancer remains a global health issue, being the third most commonly occurring cancer and the fourth most common cause of cancer-related deaths worldwide. In addition, the incidence is rising in developing countries (Favoriti et al., 2016). In a study from 2005, the development of incidence and mortality rates of CRC was compared among several countries over the last decades. While the mortality rate stagnated or slightly decreased in most countries, it has at least doubled in Japan, Hungary and Italy between 1955 and 2000. Concurrently, the authors described that the 5-year survival rates have improved since the mid 70 's. The average 5 -year survival rose from $30-40 \%$ to $45-50 \%$ in rectal cancer and from 
approximately $40 \%$ to $50 \%$ in colon cancer (Boyle and Ferlay, 2005). The variation between $\mathrm{CRC}$ frequencies among countries can partially be explained by variations in the exposure to risk factors of this disease in different regions.

\subsection{Risk factors associated with CRC}

By studying dietary and lifestyle habits, family history, molecular backgrounds, comorbidities and further CRC patient details, several CRC-related risk factors were defined. For instance, the demographic change observed in our society reflects an established CRC risk factor since it has been be shown that increasing age is correlated with the development of malignancies (Hoyert and $\mathrm{Xu}, 2012$; White et al., 2014). In fact, $50 \%$ of all diagnosed malignancies and $70 \%$ of cancer-related deaths can be observed in patients $\geq 65$ years of age (Mazzola et al., 2012). However, there are several further aspects, preventable and unpreventable, associated with increased colorectal cancer risk.

\subsubsection{Diet and lifestyle}

According to the American Institute for Cancer Research, approximately $47 \%$ of colorectal cancer cases could have been prevented if individuals would adapt their diet, activity and weight management (Baena and Salinas, 2015; Bailie et al., 2016; Ferlay, 2016; Godos et al., 2016). There are numerous reports on how this disease can be either promoted or prevented by the consumption of certain dietary factors; however, many study outcomes were inconclusive (Baena and Salinas, 2015). For instance, individuals consuming high rates of processed or red meat and alcohol have an increased colorectal cancer risk (Chan et al., 2011; Wang et al., 2015). Moreover, victuals containing high amounts of sugar were suggested to have negative effects (Galeone et al., 2012). In contrast, a fiber-rich diet, consumption of fruits, fish and milk were shown to confer a protective effect (Aune et al., 2012; Ben et al., 2014; Block et al., 1992; Yu et al., 2014). Furthermore, the composition of the bacterial community within 
the intestine can be altered by dietary changes (David et al., 2014; Turnbaugh et al., 2009). Notably, dysbiosis, i.e. dysregulation of the gut microbiota is a phenomenon frequently observed in colorectal cancer patients (Wu et al., 2013).

Importantly, regular physical activity decreased CRC risk by $24 \%$ while obesity increased it by $19 \%$ (Baena and Salinas, 2015). Obesity is a risk factor gaining significance due to its increasing frequency, especially, since it is accompanied by numerous comorbidities. In fact, the incidence of obesity has increased by $28 \%$ in adults and $47 \%$ in children since $1980(\mathrm{Ng}$ et al., 2014). Moreover, it was shown that obese individuals frequently develop type 2 diabetes (Kearns et al., 2014) which in turn is also associated with CRC as demonstrated in a recent meta-analysis (Guraya, 2015). Consistently, treating diabetic patients with the anti-diabetic drug Metformin reduced colorectal cancer risk (Zhang et al., 2011). These findings reveal how not only our diet and its effect on the microbiota can directly modulate CRC risk but also morbidities possibly resulting from inadequate nutrition or deficiency in physical activity.

\subsubsection{Inflammatory bowel diseases (IBDs)}

Currently, approximately $0.4 \%$ of Europeans and North-Americans have a high risk of developing $\mathrm{CRC}$ due to inflammatory bowel diseases (IBDs) and incidence rates are expected to rise (Molodecky et al., 2012). Generally, these conditions are characterized by a chronic inflammation of the gastrointestinal tract mediated by genetic susceptibility, environmental factors and a deregulated immune response (Mesquita et al., 2008; Renz et al., 2011; Xavier and Podolsky, 2007). Two well-described IBD types are Crohn's disease (CD) and ulcerative colitis (UC). However, besides these two major forms, there are further, rare disorders such as intestinal tuberculosis (ITB) and Behçet's disease (BD) (Lee and Lee, 2016). The so-called hygiene hypothesis was proposed to be one of the explanations for the increasing prevalence of IBDs. The rationale of this assumption is that a low exposure towards microbes during early life leads to an augmented sensitivity of the immune system towards microorganisms and 
therefore IBD (Saidel-Odes and Odes, 2014). Another hypothesis proposes the relevance of diet for the development of IBDs for instance due to a low fiber uptake (Shen and Wong, 2016). Moreover, in 2015 Liu and co-workers identified 38 loci which are associated with the susceptibility towards IBDs (Liu et al., 2015b). Symptoms of inflammatory bowel diseases can include (bloody) diarrhea, abdominal pain, passage of mucus and/or pus (Baumgart and Sandborn, 2007) and are often accompanied by psychological symptoms as anxiety or depression (Marrie et al., 2016). Upon indication, IBDs can be diagnosed via blood- and stoolbased tests, physical examination and imaging, as well as biopsy and endoscopy (Bernstein et al., 2010). Generally, IBDs have an early disease onset, i.e. $20-25 \%$ of cases are detected during childhood. Interestingly, pediatric IBDs were described to have particularly severe symptoms (Guariso et al., 2010; Ruemmele, 2010). During the course of the disease, patients frequently develop comorbidities such as anemia (Oldenburg et al., 2001), ocular manifestations (Felekis et al., 2009) as well as osteopenia and osteoporosis resulting in decreased bone mineral density (BMD) and therefore a highly increased fracture risk (Ali et al., 2009; Bernstein et al., 2000). In a meta-analysis it was determined that the overall CRC prevalence in IBD patients is $1.7 \%$. Accordingly, since disease duration correlates with the probability of $\mathrm{CRC}$ formation, for instance, the risk after $>20$ years of IBD was $5 \%$ (Lutgens, Maurice W M D et al., 2013). To reduce cancer risk, patients can be treated with antiinflammatory or immunosuppressive drugs and antibiotics. In addition, surgery may be required to remove damaged intestinal compartments (in CD patients) or even the entire colon and rectum (in UC patients), a proctocolectomy, resulting in the attachment of a pouch (Baumgart and Sandborn, 2007).

\subsubsection{Genetic susceptibility}

Nonetheless, not all CRCs can be attributed to a preexisting medical condition. In fact, it was reported that up to $30 \%$ of $\mathrm{CRC}$ cases develop due to genetic susceptibility. Approximately $5 \%$ 
of patients are affected by an inherited mutation promoting tumorigenesis (Patel and Ahnen, 2012). In the era of next generation sequencing (NGS) it would therefore be desirable to detect these genetic predispositions in order to identify individuals with a high CRC-risk to start treatment in time. It was already demonstrated that surveillance of individuals at high risk can decrease mortality rates (Jong et al., 2006).

A well-known example for a genetic alteration is familial adenomatous polyposis (FAP), an autosomal dominant disorder, affecting 1-2:10,000 newborns. This condition is characterized by a heterozygous mutation of the tumor suppressor Adenomatous Polyposis Coli (APC) resulting in a truncated and therefore non-functional protein (Rozen et al., 2001; Rozen and Macrae, 2006). Under normal conditions, APC is a negative regulator of the Wnt signaling pathway, where it is a member of the destruction complex (Figure 1A). This complex consisting of APC, the kinase GSK-3 $\beta$ and AXIN phosphorylates $\beta$-catenin to target it for ubiquitination and subsequent degradation. In the scenario of a non-functional APC protein or binding of a Wnt ligand to a Frizzled/LRP5/6 receptor, the destruction complex is destabilized (Figure 1B). Therefore, $\beta$-catenin is no longer degraded and can translocate to the nucleus where it interacts with the transcription factors Lymphoid Enhancer Factor (LEF)/T-Cell Factor (TCF). By serving as a transcriptional coactivator, accumulated $\beta$-catenin causes highly amplified cell proliferation rates (Giles et al., 2003; Reya and Clevers, 2005; Taipale and Beachy, 2001). As a consequence, hundreds to thousands of adenomas, including microadenomas, grow throughout the intestine of FAP patients (Eccles et al., 1997). In contrast, individuals affected by the attenuated form of FAP (AFAP) develop less than 100 tumors (lbrahim et al., 2014). Notably, FAP is characterized by an early colorectal cancer onset, i.e. maximum 39 years in FAP and 55 years in AFAP (Novelli, 2015). 

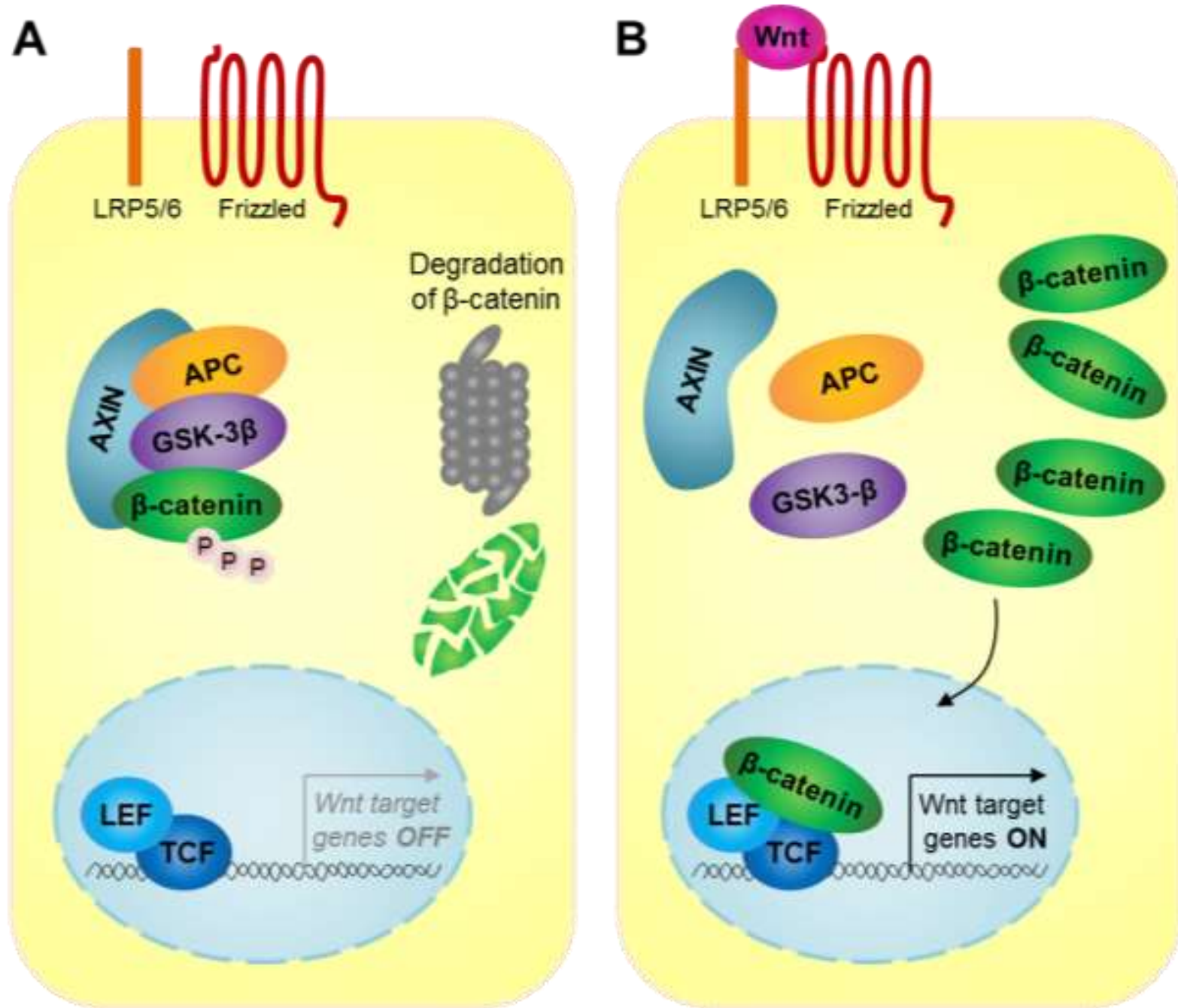

Figure 1: The canonical Wnt signaling pathway. $(A)$ In the absence of a Wnt ligand, the destruction complex consisting of GSK-3 $\beta$, AXIN and APC phosphorylates $\beta$-catenin. Thereby, $\beta$-catenin is targeted for ubiquitination and subsequent degradation by the proteasome. (B) Upon binding of a Wnt ligand to the Frizzled/LRP5/6 receptor, the destruction complex is destabilized. This destabilization is also observed if the tumor suppressor APC is nonfunctional due to a truncation mutation. Consequently, $\beta$-catenin is no longer degraded and can accumulate and translocate to the nucleus. There it can interact with the transcription factors LEF/TCF to promote transcription of Wnt target genes.

Another example for $\mathrm{CRC}$ in a familial context is the so-called Lynch syndrome which was described fifty years ago (Lynch, 1967) and accounts for approximately 3\% of all CRC cases (Hampel et al., 2008). It is an autosomal dominant disease majorly caused by germline mutations in the mismatch repair genes MutL Homologue 1 (MLH1), MutS Homologue 2 (MSH2) and 6 (MSH6) and Postmeiotic Segregation Increased 2 (PMS2) (Lynch et al., 2009). 
It should be noted that Lynch syndrome is frequently referred to as hereditary non-polyposis colorectal cancer (HNPCC). However, to be diagnosed with Lynch syndrome one must possess mutations in mismatch repair (MMR) genes while HNPCC patients/families must fulfill Amsterdam criteria $(A C)$ I or II. AC I reflects at least three CRC cases per family while AC II also takes the occurrence of extracolonic tumors into account, e.g. endometrial cancer (Novelli, 2015; Vasen et al., 1999). In Lynch patients a relatively small number $(<10)$ of polyps can be found, however, they frequently develop further malignancies such as brain, skin, ovarian and endometrial cancers (Novelli, 2015; Vasen et al., 1999). Further, but more rare examples for autosomal dominant conditions associated with an increased CRC risk are the Peutz-Jeghers syndrome $(1: 200,000)$ and Juvenile polyposis syndrome $(1: 100,000)($ Shenoy, 2016).

\subsubsection{Genetic mutations}

Besides the presence of genetic predispositions, spontaneous mutations display a frequent phenomenon in the development and progression of colorectal cancer. In 1990 several genetic driver alterations were proposed by Eric Fearon and Bert Vogelstein to be involved in the adenoma to carcinoma sequence (Fearon and Vogelstein, 1990).

The so-called Vogelgram revealed the significance of the Wnt/B-catenin pathway in the transition from normal epithelium to hyperproliferation and an early adenoma (Fearon and Vogelstein, 1990). In addition to the relevance of a functional APC protein in the case of FAP, APC mutations are the most frequent genetic alterations observed in sporadic colorectal cancer (Rozen et al., 2001; Rozen and Macrae, 2006). As mentioned before, the subsequent nuclear translocation of $\beta$-catenin results in increased cellular proliferation (Giles et al., 2003).

According to the Vogelgram, the following transition from an early to intermediate and late adenoma is mediated by disturbed RAS signaling (Fearon and Vogelstein, 1990). There are

three $\operatorname{RAS}(\mathrm{H}, \mathrm{K}$ and $\mathrm{N})$ and three $\operatorname{RAF}(\mathrm{A}, \mathrm{B}$ and $\mathrm{C})$ members. RAS is a small GTP-binding protein which is regulated by binding to GTP in exchange for GDP. When GTP is hydrolyzed, 
RAS is no longer active. A RAS mutation at the position G12 prevents the activity of a GTPase to act on RAS-GTP to deactivate it and, therefore, it remains constitutively active, leading to sustained activation of RAF signaling (Chang et al., 2003; Hallberg et al., 1994). RAF members are responsible for phosphorylating and activating Mitogen-Activated Protein Kinase Kinases 1 and 2 (MEK1/2) and Extracellular-Signal-Regulated Kinases 1 and 2 (ERK1/2) signal transduction to regulate among others MYC transcription. As a consequence, MitogenActivated Protein Kinase (MAPK) signaling is induced in which serine/threonine kinases mediate signal transduction from the plasma membrane to the nucleus (Hallberg et al., 1994; Liebmann, 2001). Notably, RAS activating mutations or amplifications resulting in constitutively active RAS proteins occur frequently in CRC, i.e. approximately $40 \%$ for KRAS and $5 \%$ for NRAS (Fearon, 2011). Interestingly, RAS can activate Nuclear Factor Kappa B (NF-kB) signaling via its downstream factor RAC or PKB/AKT (Sulciner et al., 1996). NF-KB is a transcription factor exerting anti-apoptotic effects and promoting transformation (Mayo et al., 1997). Moreover, NF-kB is associated with inflammatory processes, in general, as well as inflammation-induced gastrointestinal cancers (Merga et al., 2016). In addition to the stimulation of the MAPK and NF-kB pathways, RAS was shown to activate Phosphatidylinositol 3-Kinase (PI3K) by directly interacting with the PI3K p110 catalytic subunit (Courtney et al., 2010). Disturbed PI3K signaling was shown to be correlated with cell growth, metabolism and cancer progression (Engelman et al., 2006).

The last transition in the Vogelgram, from late adenoma to carcinoma, is characterized by the loss of the tumor suppressor TP53. TP53 stabilization is regulated via ubiquitination by the E3 ligase MDM2 resulting in its subsequent degradation. In turn, upon TP53 activation, it upregulates MDM2. Moreover, active TP53 induces mitochondrial (intrinsic) and deathreceptor-induced (extrinsic) apoptosis (Ryan et al., 2001). The corresponding gene, TP53, is mutated in approximately $50 \%$ of colorectal cancers and leads to a deregulation of the cell cycle and the prevention of apoptosis and therefore, tumor cell survival (Munteanu and 
Mastalier, 2014). Though it was shown that only $6.6 \%$ of colorectal cancer lesions possess mutations in all three components, APC, KRAS and TP53 (Smith et al., 2002), the Vogelgram has largely contributed to our knowledge and was extended by numerous studies. For instance, in 2008 Issa suggested a new model in which tumor progression does not occur in a linear progress but rather in diverse distinct pathways. He divided them based on their pathological presentation, prognosis, localization of tumors and molecular markers (Issa, 2008).

\subsubsection{Epigenetic deregulation}

Epigenetic mechanisms represent an emerging research area with significant relevance in the field of tumorigenesis (Feinberg and Tycko, 2004). Generally, epigenetic mechanisms can affect gene expression without altering underlying DNA sequences, but rather by chromatin modifications. Eukaryotic chromatin is a highly compacted structure containing repeating structural subunits, the so-called nucleosomes. Each nucleosome is assembled of a histone octamer consisting of two copies of each histone (i.e. $\mathrm{H} 2 \mathrm{~A}, \mathrm{H} 2 \mathrm{~B}, \mathrm{H} 3$, and $\mathrm{H} 4$ ) wrapped around by 147 base pairs of DNA (Luger et al., 1997; Richmond and Davey, 2003). Each core histone possesses histone-fold domains and $\mathrm{N}$-terminal histone-tails. While the fold domains serve for the interaction of the histones, the tails can be subjected to post-translational modifications (PTMs), potentially affecting gene expression. These modifications include for instance histone methylation (me), acetylation (ac), phosphorylation (P) and ubiquitination (ub). Histone PTMs can be carried out by enzymes called "writers", recognized by "readers" and removed by "erasers" (Strahl and Allis, 2000; Torres and Fujimori, 2015).

In 1983 Feinberg and Vogelstein described a global hypomethylation in human cancers (Feinberg and Vogelstein, 1983). Moreover, regions rich in CpG dinucleotides, namely the CpG islands, which are abundant in promoter regions, were found to be hypermethylated resulting in decreased transcriptional activity (Herman and Baylin, 2003). CpG hypermethylation 
frequently affects and therefore represses tumor suppressor genes in various cancer types including CRC (Costello et al., 2000).

A further relevant, yet poorly understood, epigenetic modification is the monoubiquitination of histone $\mathrm{H} 2 \mathrm{~B}$ at lysine 120 (H2Bub1). The process of H2B monoubiquitination can be initiated after the recognition of acetylated histone marks and/or transcription factors by BRD4 (Figure 2) (Itzen et al., 2014). As a consequence, BRD4, a central member of the Bromo- and ExtraTerminal (BET) domain protein family, recruits the Positive Transcriptional Elongation Factor b (P-TEFb) complex. P-TEFb consists of the Cyclin-Dependent Kinase 9 (CDK9) and Cyclin T1. P-TEF-b complex formation was shown to be facilitated by the Heat Shock Protein 90 (HSP90) (O'Keeffe et al., 2000). Subsequently, CDK9 phosphorylates the RNA Polymerase II (RNA Pol II) at serine 2 of its carboxyl-terminal heptapeptide repeat domain, thereby releasing promoter proximal pausing of several genes "poised" or "paused" by RNA Pol II (Nechaev and Adelman, 2011).

The process of promoter proximal pausing was studied in detail in Drosophila melanogaster where it was demonstrated that in $15 \%$ of tissue-specific genes RNA Pol II is bound downstream of the promoter. Moreover, nearly half of the genes in human embryonic stem cells were occupied by an initiating form of RNA Pol II (Guenther et al., 2007). In an inducible manner, this paused state can be released to proceed transcription. As reviewed by Boettiger and colleagues, it was postulated that RNA Pol II pausing allows for rapid induction upon a stimulus and was referred to as a "loaded gun". The possibility to regulate this mechanism in a tissue-specific manner implies its relevance during developmental processes (Boettiger et al., 2011). Upon phosphorylation RNA Pol II interacts with WW Domain-Containing Adaptor with Coiled-Coil (WAC) protein which recruits the RNF20/RNF40 heterodimer (Zhang and Yu, 2011). By exerting their E3 ligase activity the RING finger proteins RNF20/RNF40 monoubiquitinate H2B at lysine 120 and thereby promote transcription (Zhu et al., 2005). It was proposed that monoubiquitinated $\mathrm{H} 2 \mathrm{~B}$ facilitates transcript elongation by interacting with the 
Facilitates Chromatin Transcription (FACT) complex which promotes RNA Pol II activity (Orphanides et al., 1998; Pavri et al., 2006). Notably, the ubiquitin hydrolase Ubiquitin-Specific Protease 22 (USP22) is able to deubiquitinate H2B by functioning as a deubiquitinating enzyme (DUB) within the SAGA complex (Zhang et al., 2008b) as explained in detail in section 1.13.

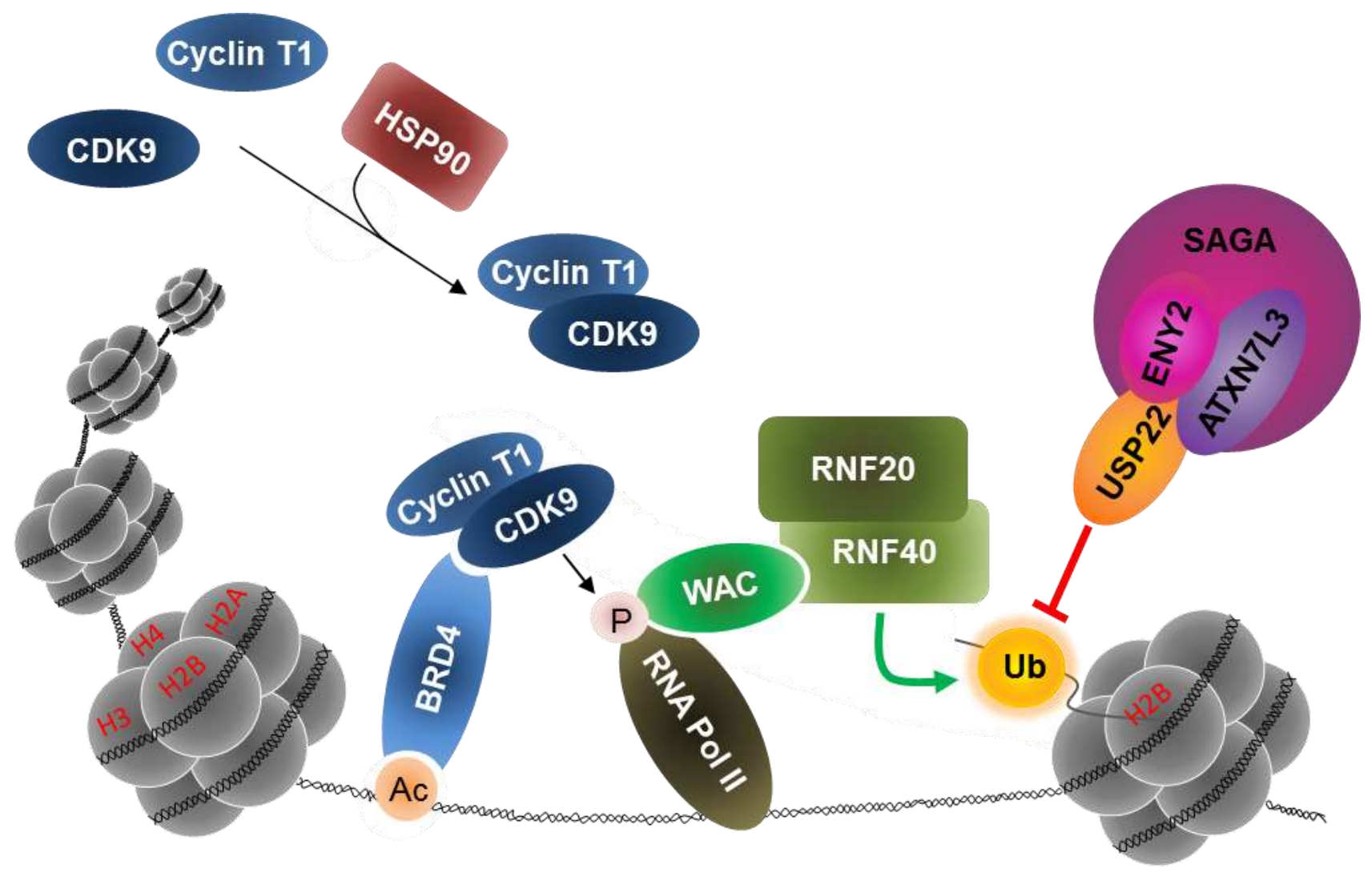

Figure 2: The interplay of H2Bub1 and epigenetic regulators. HSP9O facilitates the assembly of the P-TEFb complex consisting of CDK9 and Cyclin T1. Upon recognition of acetylated marks, BRD4 recruits the P-TEFb complex, enabling CDK9 to phosphorylate RNA Pol II at serine 2, thereby releasing promoter proximal pausing. RNA Pol II interacts with WAC which binds to the RNF20/RNF40 heterodimer monoubiquitinating the histone H2B. This ubiquitin molecule can be removed by the deubiquitinating enzyme USP22 which is part of the DUB module within the SAGA complex.

Over the last years it has been demonstrated that the levels of several components involved in the monoubiquitination of $\mathrm{H} 2 \mathrm{~B}$ are perturbed in cancer. For instance, increased abundance of BRD4 (Jung et al., 2015) and CDK9 (Morales and Giordano, 2016) have been positively correlated with tumorigenesis. Interestingly, our group previously showed that H2Bub1 levels 
are inversely correlated with malignancy during mammary tumor progression (Bedi et al., 2015; Prenzel et al., 2011) therefore potentially reflecting a tumor suppressive mark. In a recent study, 1,800 CRC samples were assessed for their H2Bub1 levels in a tissue microarray-based approach. Indeed, decreased H2Bub1 levels correlated with advanced tumor grade and stage (Qi et al., 2004). In addition, reduced Rnf20 expression in mice, and therefore decreased H2Bub1 levels, were associated with UC and CRC (Tarcic et al., 2016). Concerning the E3 ligases RNF20/RNF40, further implications in cancer were described. For instance, RNF20 appears to be required for mixed-lineage leukemia (MLL)-rearranged leukemia (Wang et al., 2013), while deletion of both RNF20 and RNF40 suppresses breast cancer progression in vivo (Duan et al., 2016). Notably, USP22 was reported to be a member of the 11-gene "death-fromcancer" gene expression signature which is strongly associated with distant metastasis, poor survival and high recurrence rates of human malignancies (Glinsky, 2005; Glinsky et al., 2005). The correlation of the overexpression of USP22 in several cancer types has been recently reviewed (Melo-Cardenas et al., 2016) and is described in more detail in section 1.13. In summary, besides well-known aspects such as lifestyle, IBDs and genetic mutations, epigenetic deregulations display substantial CRC risk factors.

\subsection{The composition of the intestinal system}

To understand how these risk factors can contribute to the transition of healthy intestinal epithelium to cancer lesions, it is of great importance to comprehend the intestinal anatomy and its cellular composition. During the last decade numerous studies have revealed the significance of the intestinal system to the human body. Its probably most well-known and best described functions are the digestion of food and the absorption of nutrients. After the uptake of food it is processed from the oral cavity and the pharynx into the upper gastrointestinal tract (GIT) consisting of esophagus and stomach (Figure 3A). From there the food reaches the lower GIT which is composed of the small and large intestine and extends to the anus (Ellis, 2006). 

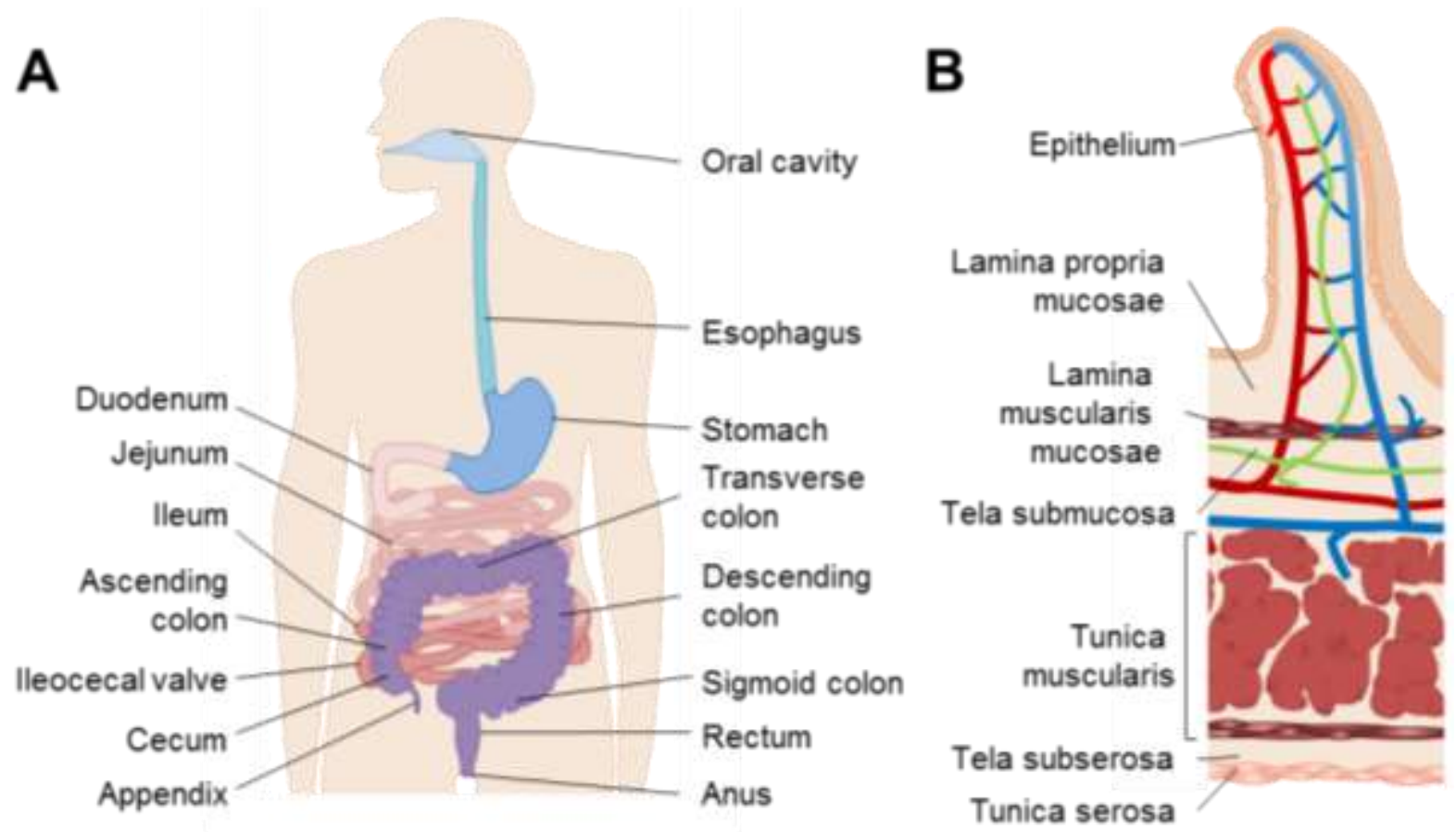

Figure 3: The human gastrointestinal tract and composition of the gastrointestinal wall. (A) After uptake, food migrates through the GIT consisting of the oral cavity, esophagus and the stomach. Afterwards, the food is further digested in the small intestine (duodenum, jejunum, ileum) and transferred to the large intestine via the ileocecal valve. The large intestine consists of the cecum, appendix, colon (ascending, transverse, descending, sigmoid) and the rectum. Finally, digestion is completed by defecation via the anus. (B) The intestinal lumen is surrounded by epithelial cells with the subjacent lamina propria mucosae. Below, the smooth muscle layer lamina muscularis mucosae and the tela submucosa can be found. Underlying the tunica muscularis, tela subserosa, and the outermost connective tissue layer, tunica serosa, are present.

Generally, after receiving food from the stomach, the main function of the small intestine (SI) is to digest the food and absorb substances such as lipids, carbohydrates and vitamins into the bloodstream or lymphatic system. The SI is structurally subdivided into the duodenum which is continuous with the stomach, the jejunum, and the ileum. The uppermost $25 \mathrm{~cm}$ of the SI represent the duodenum which performs the major part of digestion with the help of chyme, digestive enzymes and bile contributed by stomach, pancreas and gall bladder, respectively. In the jejunum mainly fatty acids, sugars and amino acids are absorbed while in the ileum mainly absorption of bile acids, vitamins and further nutrients takes place. To increase the surface area, there are circular folds within the intestinal system. The large intestine is divided 
into further structural subunits, namely the cecum, colon, rectum and the anal canal. The cecum is connected to the small intestine and is separated from the ileum by the ileocecal valve. The appendix is a blind, tube-formed structure attached to the cecum and plays a crucial role in mucosal immunity due to its high density of lymphocytes (Berry, 1900). The following structure is the colon which is divided into the ascending, transverse, descending and sigmoid colon. The main function of the colon is to absorb water from the food residues. The rectum is the final straight segment which is followed by the anal canal and the anus where the process of digestion is completed by defecation (Despopoulos and Silbernagl, 2003; Ellis, 2006; Rhoades and Tanner, 2003). Typically, the intestinal tissue possesses finger-like projections to further increase its surface. In the small intestine these evaginations are designated as villi with crypts at their bottom while the colon contains only invaginations (crypts). Generally, the lumen is surrounded by a monolayer of epithelial cells with an underlying layer of collagenous connective tissue, the lamina propria mucosae (Figure 3B). Below the smooth muscle layer lamina muscularis mucosae is present as well as the tela submucosa which contains nerves, blood and lymphatic vessels. The tunica muscularis is composed of longitudinal and circular muscle layers responsible for peristaltic movements of the intestine. Underlying, a layer of connective tissue, tela subserosa, and the outermost connective tissue layer, tunica serosa, can be found (Gartner and Hiatt, 2014; Young and Wheater, 2007).

Interestingly, the intestinal epithelium is characterized by a rapid cell turnover with a renewal every 4-5 days and is therefore the most frequently self-renewing tissue in adults (van der Flier, Laurens $G$ and Clevers, 2009). It consists of a monolayer of cells interconnected by tight junctions. Importantly, these tight junctions regulate the epithelial barrier function, i.e. permeability for solutes, ions, cells, etc. through intercellular space (Ichikawa-Tomikawa et al., 2011). Epithelial cells are subdivided into four differentiated cell types (Figure 4). The majority of cells are enterocytes which are responsible for absorption. Goblet cells secrete mucins which are required to form a mucus layer in the intestine which confers a protective role to the organ. 

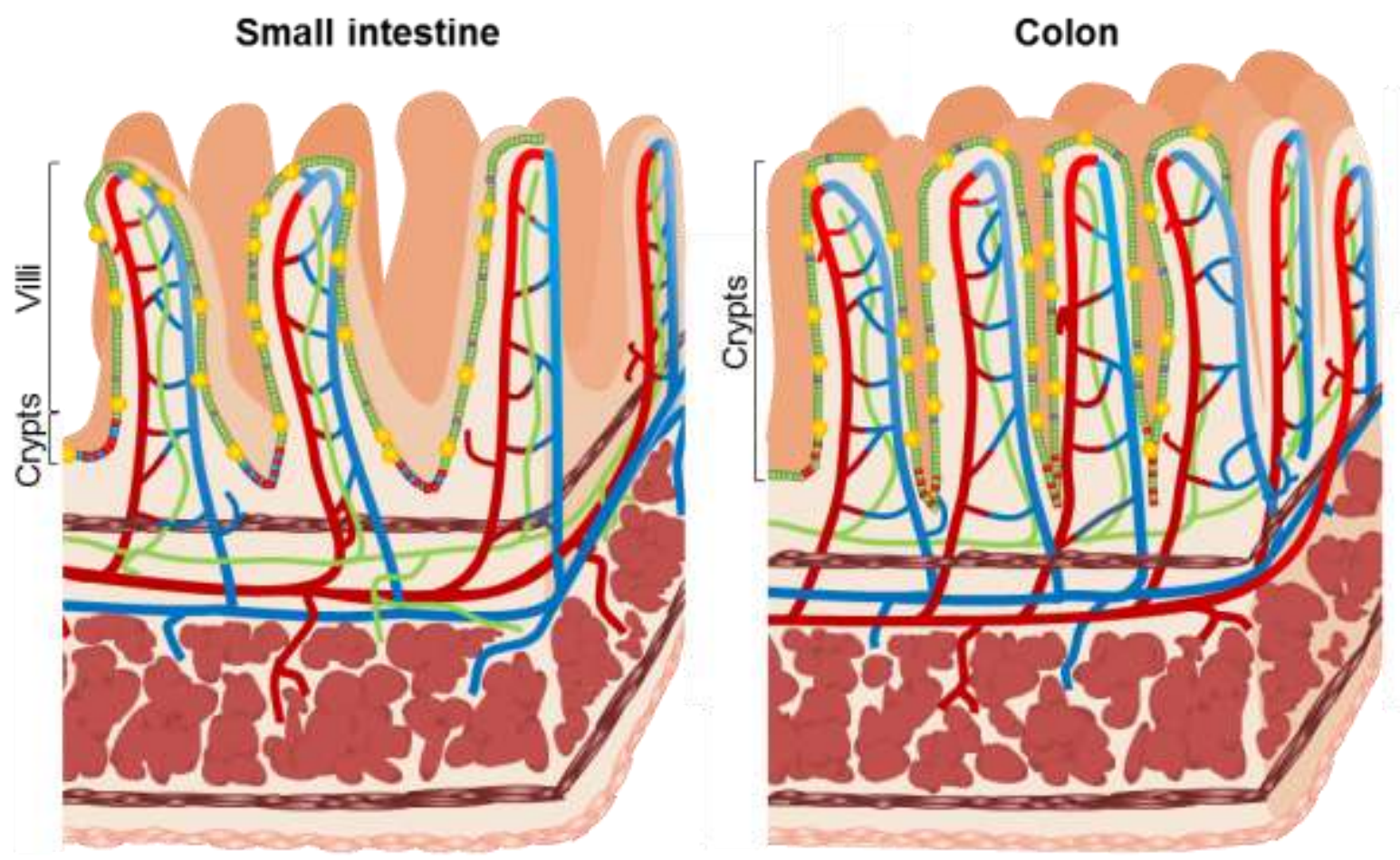

Of Enterocytes

Goblet cells

(0) Stem cells

\section{Paneth cells 0 Enteroendocrine cells}

Figure 4: Structure and cell types of the intestinal epithelium. The small intestine is subdivided into finger-like projections, villi, and crypts which are found at the bottom of villi. A monolayer of epithelial cells faces the luminal area of the intestinal tube. The epithelium mainly consists of enterocytes while Goblet and enteroendocrine cells are sporadically present. At the bottom of the crypts, Paneth and stem cells are located. In contrast, the colon consists only of crypts and no Paneth cells are present.

Enteroendocrine cells secrete hormones which are amongst other functions relevant for digestion. In contrast to the other cell types, Paneth cells are exclusively present at the bottom of the crypts and secrete antimicrobial substances in addition to the fact that they are exclusively found in the small intestine but not the colon (Johansson et al., 2011; Roda et al., 2010). Most importantly, the crypt bottoms harbor stem cells which give rise to the so-called transit-amplifying cells which migrate upwards into the villi to differentiate into the previously mentioned cell types (Barker et al., 2007; Barker et al., 2012; van der Flier, Laurens G et al., 2009). The differentiation process is strongly regulated by active Wnt and Notch signaling 
pathways which are also responsible for keeping the crypt compartment in an undifferentiated and proliferative state. Consistent with our knowledge about tumorigenesis, Barker and coworkers demonstrated that loss of APC in intestinal stem cells causes uncontrolled proliferation and therefore development of adenomas (Barker et al., 2009). Interestingly, epigenetic mechanisms appear to be crucial for the maintenance of the intestinal epithelium. For instance, the loss of the maintenance DNA methyltransferase 1 (DNMT1) resulted in an almost twofold expansion of the crypt compartment in mice. This phenomenon was accompanied by increased expression of the stem cell markers Lgr5 and Olfm4 (Sheaffer et al., 2014). The fact that enhancer regions of these genes were strongly demethylated suggested a role of epigenetic regulation in differentiation and crypt maintenance in vivo (Lister et al., 2009).

\subsection{The intestinal microbiota}

To understand the complexity of physiological and pathological processes in the colon, it is relevant to know that the intestinal system should not be seen as an empty tube through which food is processed and in which tumors can develop. In fact, it displays a highly complex ecosystem with its own microenvironment, immune response and if malfunctioning, it can affect the entire body. In the process of digestion our body relies on numerous microorganisms, the so-called microbiota. Not only does the microbiota mainly comprise bacteria, but also fungi, viruses and protozoans can be found (Savage, 1977). One gram of dry ileal contents contains approximately $10^{8}$ bacteria while the colon contains up to $10^{12}$ bacteria per gram (Smith, 1977). Notably, each human being possesses at least 160 species while in the entire experimental cohort analyzed by Qin and co-workers more than 1,000 different species were found (Qin et al., 2010).

The most abundant phyla in the intestines gut are Firmicutes and Bacteriodetes (Human Microbiome Project Consortium, 2012), however, the composition of the gut microbiota is highly variable. For instance, the microbiota can vary among human populations, across age 
(Yatsunenko et al., 2012), after antibiotic treatment (Panda et al., 2014) or due to dietary factors (David et al., 2014; Turnbaugh et al., 2009). As explained in detail in section 1.5, the microbiota can be seen as a component of the natural defense barrier of the intestine, however, at the same time it represents a potential danger in the case of dysbiosis. In dysbiosis, the microbial homeostasis is no longer maintained resulting in an increase of pathogenic and a decrease in beneficial microorganisms (Comito et al., 2014) and was described in colorectal cancer (Wu et al., 2013) and IBD patients. For instance, in IBD a high frequency of Bacteroides sp. was found (Rehman et al., 2010; Swidsinski et al., 2002) which produce sulfatases degrading the protective mucin layer resulting in impaired barrier function of the epithelium (Lucke et al., 2006). Interestingly, the presence of microorganisms is indispensable for the development of factors of the immune response and even the gut morphology (Round and Mazmanian, 2009; Zeissig and Blumberg, 2013).

\subsection{The intestinal immune defense and ulcerative colitis}

In addition to the intestinal microbiota, our intestinal system is constantly confronted with potentially pathogenic food- and water-born agents. Hence, it must possess multiple defensive mechanisms which can involve physical, chemical, cellular or signaling processes. Inside the intestinal lumen the microbially-colonized mucus layer (Figure 5) protects the underlying epithelium in a dual way. First, the presence of microorganisms protects its host from bacterial overgrowth and infection with enteropathogenic bacteria via competition for nutrients and space (Frick and Autenrieth, 2013). Most of these bacteria possess a beneficial role in the organ function, for instance by their metabolic contributions (Hooper et al., 2002) but also by producing anti-microbial compounds in the presence of pathogens (Frick and Autenrieth, 2013). Second, also the mucus layer prevents bacterial overgrowth and protects the epithelium from inflammation at the same time (Petersson et al., 2011). Notably, mice deficient in Muc2, a main component for mucin formation, were prone to the development of colitis (van der Sluis 
et al., 2006). A physical defense component is represented by the monolayer of intestinal epithelial cells (IECs) including tight junctions and microvilli to increase the surface area.

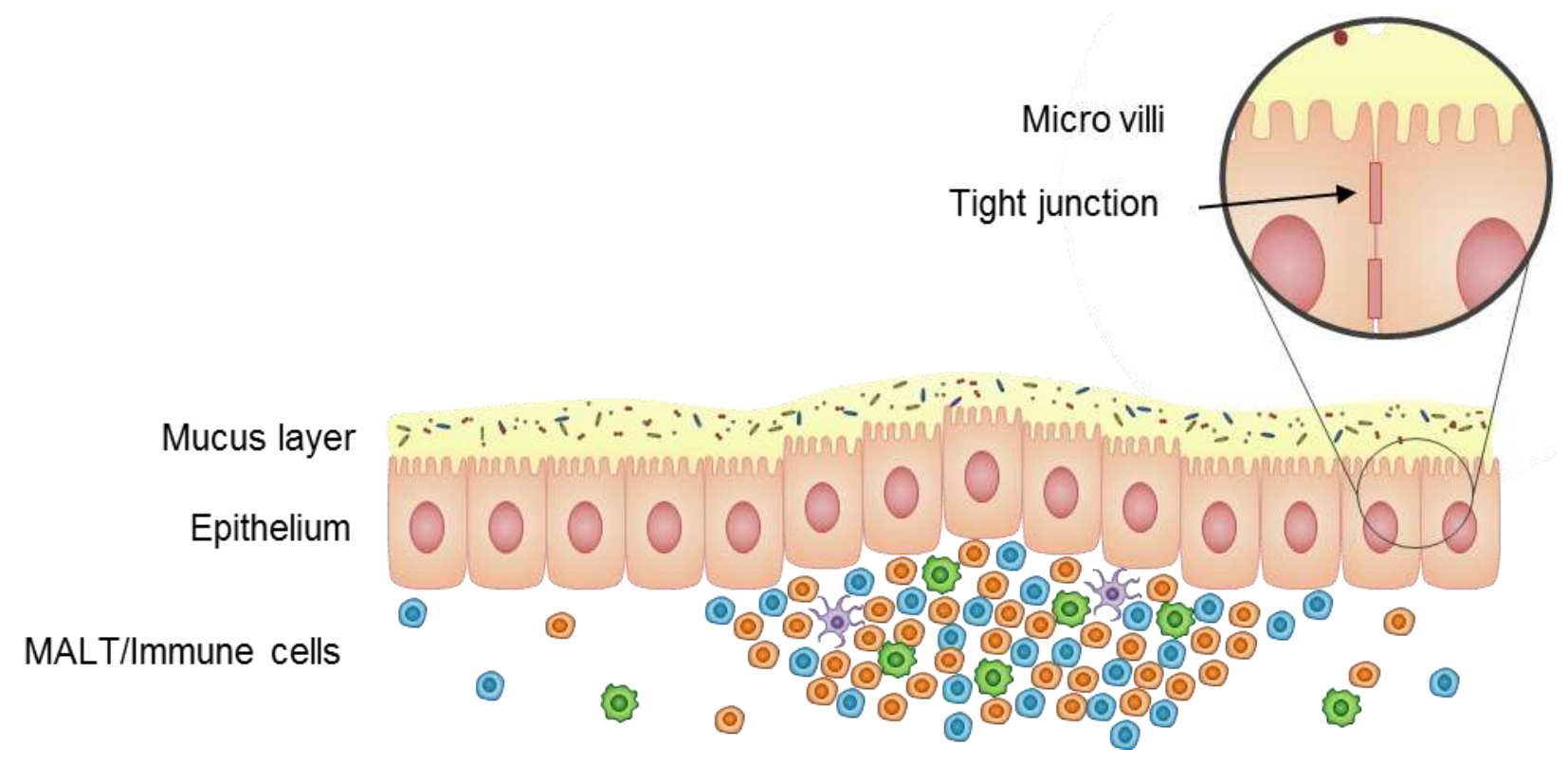

Figure 5: The protective structures of the intestinal system. The mucus layer represents the first defensive component facing the intestinal lumen. Apart from its protective and lubricant function, it also harbors numerous microorganisms protecting the host from bacterial overgrowth. The epithelial monolayer represents a physical barrier which contains microvilli to increase surface area and tight junctions to regulate permeability. Below, free immune cells and mucosa-associated lymphoid tissues (MALTs) are responsible for the host's immune response.

As mentioned before, tight junctions regulate the paracellular barrier function via several transmembrane proteins (Ichikawa-Tomikawa et al., 2011). Another crucial feature of this cell layer is the presence of granule-containing Paneth cells which secrete anti-microbial substances such as defensins (Clevers and Bevins, 2013). Importantly, a certain degree of permeability is maintained in order to transport nutrients and fluids across this barrier. Below the physical barrier, mucosal immunity is regulated by free lymphocytes, dendritic cells, Natural killer T cells and macrophages which are in close proximity to IECs. Similar to the microbiota, Natural killer T cells can play a beneficial role by protecting the host from infections, however, deregulated activation can contribute to the pathogenesis of IBDs (Zeissig et al., 2007). Moreover, mucosal structures harbor mucosa-associated lymphoid tissues (MALTs) which in 
the case of the intestine are known as gut-associated lymphoid tissue (GALT) such as Peyer's patches (Mora and Andrian, 2008). Interestingly, in 1900 the significance of the appendix in our immune defense was recognized since lymphoid tissue is highly concentrated in this structure (Berry, 1900). In these lymphocyte accumulations, B cells can differentiate into Immunoglobulin A ( $\lg A)$-secreting cells (Mora and Andrian, 2008), an antibody of the innate immune system interfering with the invasion of bacteria into the IECs (Canny and McCormick, 2008). In fact, $\lg A$ is the most abundant immunoglobulin isotype in the human body and approximately $80 \%$ of $\lg \mathrm{A}$-secreting cells are localized in the intestinal mucosa (Mora and Andrian, 2008). Moreover, a subset of immune cells is able to promote expression of proinflammatory cytokines such as interleukin 6 (IL-6) and IL-8 but also of anti-inflammatory factors, e.g. IL-10 (Sommer and Backhed, 2013). As reviewed by Klampfer, cytokines and chemokines promote the proliferation and survival of tumor cells (Klampfer, 2011). In inflammatory bowel disease the intestinal barrier function was shown to be perturbed (Arslan et al., 2001; Hollander et al., 1986). Intestinal permeability can be affected by several factors such as misbalanced abundance of tight junction proteins (Barmeyer et al., 2015), damaged epithelium (Clayburgh et al., 2004) and increased abundance of inflammatory cytokines (Neurath, 2014). As recently discussed, it is challenging to determine the causal factor. Does barrier dysfunction cause inflammation? Or is it the other way around, or both, resulting in a vicious circle (Landy et al., 2016)? Once the permeability of the epithelial monolayer is disturbed luminal antigens and potentially pathogenic microorganisms can invade the mucosa triggering the innate and adaptive immune response (Rakoff-Nahoum and Bousvaros, 2010). Interestingly, while in ulcerative colitis only the mucosa is affected, inflammation in Crohn's patients appears to be transmural, i.e. affecting all layers (Xavier and Podolsky, 2007).

How inflammatory processes ultimately promote intestinal tumorigenesis, is a highly complex process and was explored several studies. For instance, the proinflammatory cytokine IL-1 $\beta$ produced by macrophages, was reported to be associated with the phosphorylation and 
therefore inactivation of GSK-3ß. Thereby, the Wnt signaling pathway was promoted by a NFKB-mediated activation of Phosphoinositide-Dependent Kinase-1 (PDK1) and the protein kinase PKB/AKT (Kaler et al., 2009). Notably, AKT can promote the nuclear translocation of $\beta$ catenin and $\beta$-catenin/TCF transcriptional activity (Fang et al., 2007) which was associated with colorectal tumorigenesis (Taipale and Beachy, 2001). Similarly, it was demonstrated in vivo that PI3K activity is required for AKT-mediated activation of Wnt signaling (Lee et al., 2010). Interestingly, it was observed that intestinal inflammation can cause mutations. For instance, macrophages and neutrophils, which are recruited during inflammatory processes, were shown to produce reactive oxygen and nitrogen species (RONS). In a mouse model for colitis, RONS were associated with the induction of DNA damage and mutations and an increased CRC risk (Meira et al., 2008). Notably, when analyzing non-cancerous colon regions isolated from UC patients, high TP53 mutation rates were identified (Hussain et al., 2000). Consistently, as reviewed by Rogler, TP53 mutations occur during the early phase in colitisassociated colorectal cancer while this phenomenon can usually be observed in the late stages of sporadic CRC. Afterwards, dysplastic lesions accumulate further mutations, e.g. in KRAS or APC, which consequently lead to the formation of a carcinoma (Rogler, 2014). Notably, it was observed that the prognosis of ulcerative colitis-associated colorectal cancer is worse than in sporadic CRC (Jensen et al., 2006).

\subsection{Colorectal tumor progression}

Generally, the formation of colorectal cancer is a multistep process characterized by its slow progression. In the majority of patients, tumors develop after a minimum of ten years allowing for early detection if screenings are performed regularly (Smith et al., 2001). Usually, CRC is initiated by a hyperproliferation of the intestinal epithelium (Figure 6). 


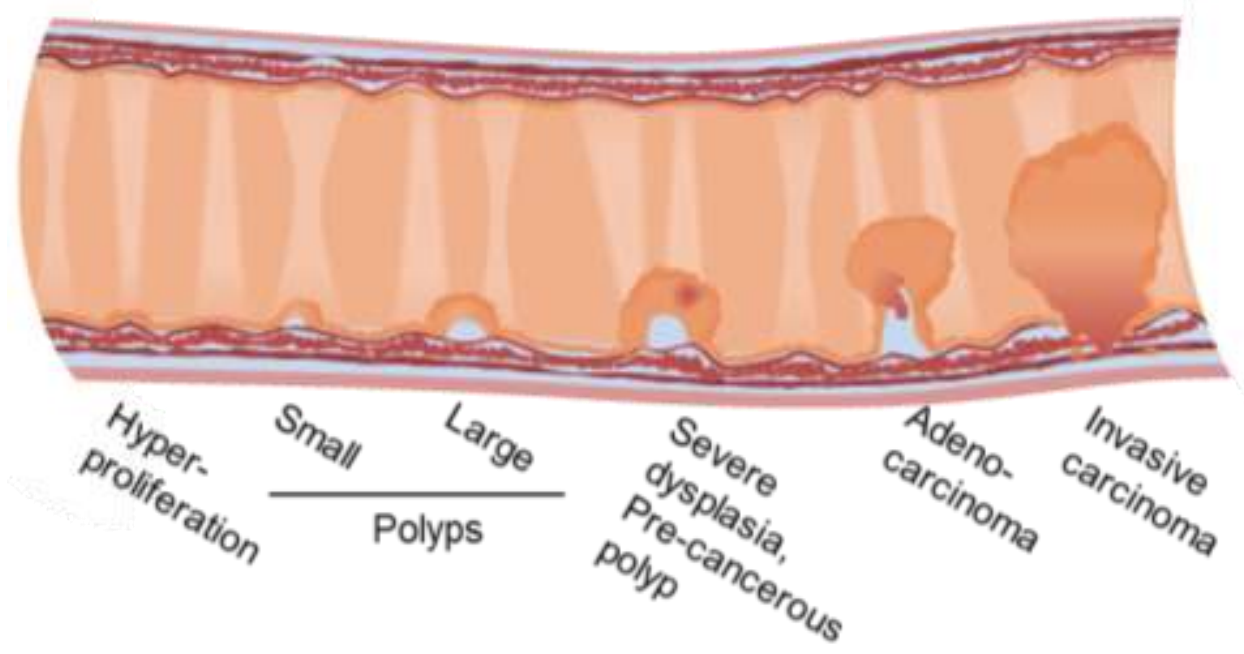

Figure 6: Tumor progression in colorectal cancer. Once mutated cells started hyperproliferating, they can form polyps. After accumulating mutations and proliferative properties pre-cancerous polyps followed by adenomas and adenocarcinomas occur. Finally, an invasive carcinoma can be formed which migrates through lamina propria and muscularis mucosae and can finally form metastases.

Notably, tumors can have different cells of origin, e.g. stem-like, transient-amplifying, Gobletlike and enterocytes (Sadanandam et al., 2013). If these hyperplastic properties are maintained, benign polyps of different sizes can be formed. Pre-cancerous polyps characterized by severe dysplasia can be developed as well as adenomas or adenocarcinomas. Finally, the cancer cells can gain migratory properties resulting in the formation of invasive adenocarcinoma (Janne and Mayer, 2000; Subramaniam et al., 2016). Tumors can invade through the lamina propria and muscularis mucosae into the proximity of the lymphatic system blood vessels (Fleming et al., 2012). Once cancer cells have reached the circulation, they can form lymphatic metastases or be transported to other organs. Liver metastases were detected in $10-25 \%$ of CRC patients who underwent surgery (Sheth and Clary, 2005). Importantly, the location of the primary tumor can be a determinant for the disease outcome. If the cancerous lesion is located in the rectal region, metastases form frequently in thorax, bone and nervous system. In contrast, in tumors located on the right side of the colorectum, metastases are more likely to form in the peritoneum and liver (Riihimaki et al., 
2016). Generally, the right sided tumors were reported to be more aggressive and therefore correlated to poor prognosis (Hemminki et al., 2010).

\subsection{Colorectal cancer detection}

In order to improve prognosis and to prevent further tumor progression, early detection remains a critical aspect. Besides the previously mentioned colonoscopy there are further less invasive CRC screening approaches. Generally, two major types of screening methods, namely stoolbased and endoscopic/radiologic tests are available. The former include the guaiac-based fecal occult blood test (gFOBT), fecal immunochemical test (FIT), and fecal DNA testing. The latter include among others optical colonoscopy, capsule endoscopy and computed tomographic colonography (CTC) and magnetic resonance colonography (MRC) (El Zoghbi and Cummings, 2016; Kolligs, 2016). In gFOBT, the presence of occult blood can be detected after applying feces on a guaiac-coated test paper. After the addition of hydrogen peroxide, an oxidation reaction takes place resulting in the development of blue color in the presence of occult blood (Mandel et al., 1993; Young et al., 2015). In contrast, the FIT makes use of antibodies specifically recognizing the globin moiety of human hemoglobin (Mandel et al., 1993; Young et al., 2015). A recent meta-analysis describes a higher sensitivity of immunochemical approaches compared to gFOBT (Launois et al., 2014). The stool DNA test (Cologuard) is a relatively new technique which combines a FIT-based detection of human hemoglobin with a molecular screen for CRC-associated biomarkers (The Medical Letter, 2014).

Colonoscopy is a standard endoscopic approach to directly visualize the intestinal structures and remove potential pre-cancerous polyps using a flexible endoscope. In case of colonoscopy contraindications or failure, capsule endoscopy can be performed (e.g. PillCam 2; Given Imaging Ltd., Israel). After capsule ingestion, a series of images is generated via two cameras which is subsequently evaluated by a gastroenterologist to detect diverticulosis, colitis, polyps or cancer lesions (Choi et al., 2016; Friedel et al., 2016). CTC is a radiographic method in 
which a contrast medium is applied to patients in order to obtain images. Detection rates are similar to colonoscopy while CTC resulted in considerably less complications (Kim et al., 2007). Though MRC was shown to be less sensitive than conventional colonoscopy (Graser et al., 2013) and CTC as recently reviewed (Kolligs, 2016), its advantage is the absence of radiation risk compared to CTC (Brenner, 2004).

\subsection{Classification of CRC subtypes}

Upon detection of colorectal cancer and the analysis of biopsies, the tumors can be classified based on, for instance, their histopathological and molecular features.

\subsubsection{Histopathological classification}

Due to the heterogeneity of colorectal cancer, e.g. the different stages and distinct pathological features, there are several histopathological classification criteria. For instance, based on their architecture, conventional adenomas can be classified as tubular, villous and tubulovillous lesions. Tubular adenomas are characterized by crypt-like dysplastic glands and a low (<25\%) presence of finger-like projections, i.e. villus-like structures. In contrast, the rate of villous component is at least $75 \%$ in villous adenomas. Accordingly, tubulovillous adenomas show intermediate rates of villous components. Furthermore, serrated lesions are characterized by a serrated or sawtooth-like presentation of the epithelium (Fleming et al., 2012).

The World Health Organization (WHO) has classified colorectal carcinomas into several subgroups such as adenocarcinomas, mucinous adenocarcinomas (MACs) and Signet ring cell adenocarcinomas. Conventional adenocarcinomas are characterized by the presence of glandular structures based on which the tumor can be graded. A high rate of glands (>95\%) represents a well differentiated adenocarcinoma while a low rate $(<50 \%)$ indicates a poorly differentiated adenocarcinoma. In contrast, cancer lesions are designated as mucinous adenocarcinomas when at least $50 \%$ of the tumor volume consists of extracellular mucin. When 
the mucus content is less between $10 \%$ and $50 \%$, tumors are described as adenocarcinomas with mucinous features/differentiation (Hamilton S.R., 2000). A meta-analysis revealed that MACs are majorly found in the right colon and are less frequent in male patients. Moreover, the authors described a poor prognosis for CRC patients with MACs (Verhulst et al., 2012). Mucinous adenocarcinomas are associated with microsatellite instability (MSI) and therefore often present in HNPCC or Lynch syndrome patients. Interestingly, MSI-MACs were described to be less aggressive than microsatellite stable (MSS) lesions (Leopoldo et al., 2008; Verhulst et al., 2012).

Excessive mucus is also present in another type of adenocarcinoma, i.e. Signet ring cell adenocarcinoma. In affected cells large intracytoplasmic mucin vacuoles push the nuclei aside. At least $50 \%$ of tumor cells should possess Signet ring features to classify a cancer lesion as a Signet ring cell adenocarcinoma (Hamilton S.R., 2000). The presence of these tumors result in a worse overall outcome compared to conventional adenocarcinomas (Kang et al., 2005). There are further classifications such as medullary carcinomas which are extremely rare (Thirunavukarasu et al., 2010) with 5-8:10,000 CRC patients and associated with MSI (Hinoi et al., 2001). Finally, the staging according to the TNM classification of colorectal cancer does not only depend on the nature of the primary tumor $(T)$, but also on the degree of invasion of regional lymph nodes $(\mathrm{N})$ and distant metastasis $(\mathrm{M})$. Each category has several subclasses, for instance, the size/spread of the primary tumor (T1-4), the number of affected lymph nodes (N0-2) and the presence of distant metastases (M0/1) (Akkoca et al., 2014).

\subsubsection{Molecular classification}

Besides the histopathological analysis, the molecular profile of the tumor biopsy is usually determined. Based on mutations in (proto-)oncogenes, tumor suppressors and further molecular alterations, a classification of the molecular basis of colorectal cancer into three pathways was proposed (Ahnen, 2011). In lesions characterized by the chromosomal instability 
(CIN) pathway, large chromosome regions or entire chromosomes are depleted or translocated. This phenomenon results in the dysfunction of relevant tumor suppressor genes or proto-oncogenes (Coppede et al., 2014). The CIN pathway is among others associated with deregulated protein levels of APC, KRAS, and TP53 (Pancione et al., 2012). In contrast to CINpositive cancer lesions, tumors displaying microsatellite instability are characterized by changes in short base pair sequences. These deletions, insertions or substitutions of nucleotides lead to a significant genetic instability and frequently affect DNA mismatch repair genes. MSI can be detected in approximatively $15 \%$ of all colorectal tumors which commonly show a mucinous phenotype (Ahnen, 2011; Vilar and Gruber, 2010). Finally, the epigenetic pathway involves gene expression alterations not resulting from changes in the DNA sequence. Over the last years, major advances have been made in this rapidly evolving field helping researchers to unravel the role of epigenetic regulatory proteins in cancer (Ahnen, 2011; Mishra and Johnsen, 2014; Okugawa et al., 2015). For instance, the CpG island methylator phenotype (CIMP) subtype of CRC displays high rates of hypermethylated tumor suppressor genes which correlates with poor prognosis (Juo et al., 2014; Toyota et al., 1999).

This molecular classification has been further refined in several studies. In 2015 an international consortium defined four consensus molecular subtypes (CMS) in which of MSI, CIN and CIMP can be accompanied by specific genetic mutations or processes such as immune or stromal infiltration. CMS1 combines MSI and CIMP with immune infiltration, while CMS2 includes copy number alterations and activation of MYC and Wnt signaling pathways. In contrast, CMS3 is associated with mixed MSI status, KRAS mutations and the deregulation of metabolic processes. Finally, in CMS4, copy number alterations, active Transforming growth factor- $\beta$ (TGF- $\beta$ ) signaling, stromal infiltration and angiogenesis can be observed (Guinney et al., 2015). 


\subsection{Colorectal cancer treatment}

The diversity of CRC underlines the importance of tumor characterization to find the most suitable therapy option upon diagnosis. The major treatment options include surgery, radiation therapy, chemotherapy and targeted therapy (Cunningham et al., 2010). Generally, if the cancerous lesions are limited to only one location, total resection of the tumor including margins of at least $5 \mathrm{~cm}$ is performed. Additionally, at least 12 lymph nodes are typically removed for nodal staging. Total mesorectal excision (TME) for rectal and complete mesocolic excision (CME) for colon cancer describe the complete removal of the respective organ including the mesentery. Via additional excision of the mesentery, a membranous fold attaching colon/rectum to the abdominal wall, surgeons can ensure that cancerous mesenteryassociated lymph nodes will be removed as well. This procedure potentially reduces tumor recurrence after curative resection (Dimitriou and Griniatsos, 2015).

If oncogenic growth is not restricted to one area due to venous or lymphatic invasion, radiotherapy combined with chemotherapy, i.e. chemoradiation, is the option of choice (Cunningham et al., 2010; Hafner and Debus, 2016). Notably, preoperative chemoradiation was associated with low recurrence rates and toxicity compared to postoperative treatment (Sauer et al., 2004). Notably, numerous studies revealed that CRC patients benefit more from combination therapies than from treatment with a single chemotherapeutic agent in terms of overall survival and tumor progression. For instance, the combinations of folinic acid and Oxaliplatin (FOLFOX) or folinic acid and Irinotecan (FOLFIRI) emerged as standard therapies (Aschele et al., 2011; Gramont et al., 1997; Landre et al., 2015; Mohiuddin et al., 2006). Notably, combining all three mentioned agents, folinic acid, Oxaliplatin and Irinotecan (FOLFOXIRI) resulted in better response as well as higher progression-free and overall survival compared to FOLFIRI (Falcone et al., 2007).

Interestingly, targeted therapy, i.e. monoclonal antibodies, was shown to improve treatment efficacy when combined with standard regimens in metastatic colorectal cancer. Bevacizumab, 
selectively targets the Vascular Endothelial Growth Factor (VEGF) and therefore impairs angiogenesis (Ohhara et al., 2016). For example, when administering Bevacizumab to patients receiving FOLFIRI, beneficial effects on the progression-free survival were noted (Fuchs et al., 2007). Similar observations were made when combining Cetuximab targeting the Epidermal Growth Factor Receptor (EGFR) with standard therapies (Ohhara et al., 2016).

Another promising approach is the induction of synthetic lethality. Synthetic lethality is based on the concept that the presence of a 'first hit' (e.g. genetic mutation) does not affect cell survival. In fact, certain mutation can rather result in a growth advantage for cancer cells. However, introduction of a 'second hit' (e.g. drug treatment or a second mutation) causes lethality in the mutated but not in normal, wild type cells (Kaelin, JR, 2005). One well-known example is the application of Poly(ADP-Ribose) Polymerase (PARP) inhibitors in patients with a mutation in BReast CAncer 1 or 2 (BRCA1 or BRCA2). BRCA1/2 contribute to DNA doublestrand break (DSB) repair (Yoshida and Miki, 2004) while PARP is necessary for the repair of single-strand breaks (SSBs) (Dantzer et al., 2000). The rationale behind this treatment is that once PARP is absent, SSBs develop to DSBs and since BRCA1/2 are not functional, these DSBs cannot be repaired. In other words, BRCA1/2 mutated cancer cells solely depend on PARP-mediated repair and once this mechanism is inhibited, lethality is induced. In contrast, healthy, BRCA1/2 wild type cells are not significantly affected by PARP inhibition since their DSB repair mechanism is still effective (Murata et al., 2016).

In addition to conventional therapies and the induction of synthetic lethality, several epigenetic approaches are currently being tested in clinical trials or have already been FDA-approved. For instance, alterations in the methylation equilibrium can be targeted via DNA methyltransferase inhibitors, e.g. 5-aza-2'-deoxycytidine (Ghoshal et al., 2005). Histone methyltransferase inhibitors such as EPZ-6438 targeting Enhancer of Zeste Homolog 2 (EZH2) as well as histone demethylase inhibitors are also currently tested in clinical trials (Mair et al., 2014). Furthermore, suberoylanilidehydroxamic acid (SAHA/Vorinostat), an inhibitor of histone 
deacetylases (HDACs) was shown to be effective in T cell lymphomas by maintaining the levels of acetylated lysines and therefore normalizing gene expression patterns (Bose et al., 2014). Interestingly, in non-small cell lung cancer this epigenetic inhibitor improved the efficacy of the chemotherapeutic agents carboplatin and paclitaxel (Ramalingam et al., 2010). Another example is the combination of the HDAC inhibitor panobinostat with the proteasome inhibitor bortezomib in multiple myeloma patients (San-Miguel et al., 2014). Notably, the progressionfree survival was increased by additional treatment with dexamethasone (Richardson et al., 2016). A further promising group of epigenetic drugs is represented by Bromo- and ExtraTerminal domain inhibitors, e.g. JQ1 and I-BET151. As recently summarized by Ferri et al., there are several BET inhibitors being tested in clinical studies for malignant, inflammatory and cardiovascular diseases. Moreover, synergistic effects were observed when combining these inhibitors with HDAC or kinase inhibitors as well as conventional chemotherapeutic agents in vivo and in vitro (Ferri et al., 2016). A BET-associated candidate, CDK9 which is recruited by BRD4 in order to release promoter proximal pausing, is currently also under investigation as a suitable drug target (Bose et al., 2013). However, despite a successful tumor removal or chemo- and radiotherapy, disease recurrence and metastasis remain major challenges.

\subsection{Heterogeneity of colorectal cancer}

One of the challenges in investigating colorectal cancer and generating adequate therapy options is reflected by the heterogenic nature of this disease. In this section all aforementioned details about CRC are summarized. Generally, there are several risk factors associated with CRC formation (Figure 7). For instance, several morbidities were described such as type 2 diabetes, obesity and, importantly, inflammatory bowel diseases. In addition, dietary and life style factors, e.g. alcohol consumption, smoking and the degree of physical activity were identified as risk factors. Individual parameters such as age and familial predisposition are correlated with colorectal cancer as well. 
Once the cancer has formed from a certain cell of origin (e.g. stem-like, transient amplifying, Goblet-like, enterocyte) there are various parameters by which this disease can be characterized. Initially, it is relevant to determine whether the CRC is of sporadic, hereditary or inflammation-induced nature. The developing tumors can be affected by genetic or epigenetic alterations and based on these, the molecular subtype (CIN, MSI, CIMP) can be determined. These underlying molecular features can influence the tumor architecture which can be of serrated, tubular, villous or tubulovillous nature and either differentiated or undifferentiated.
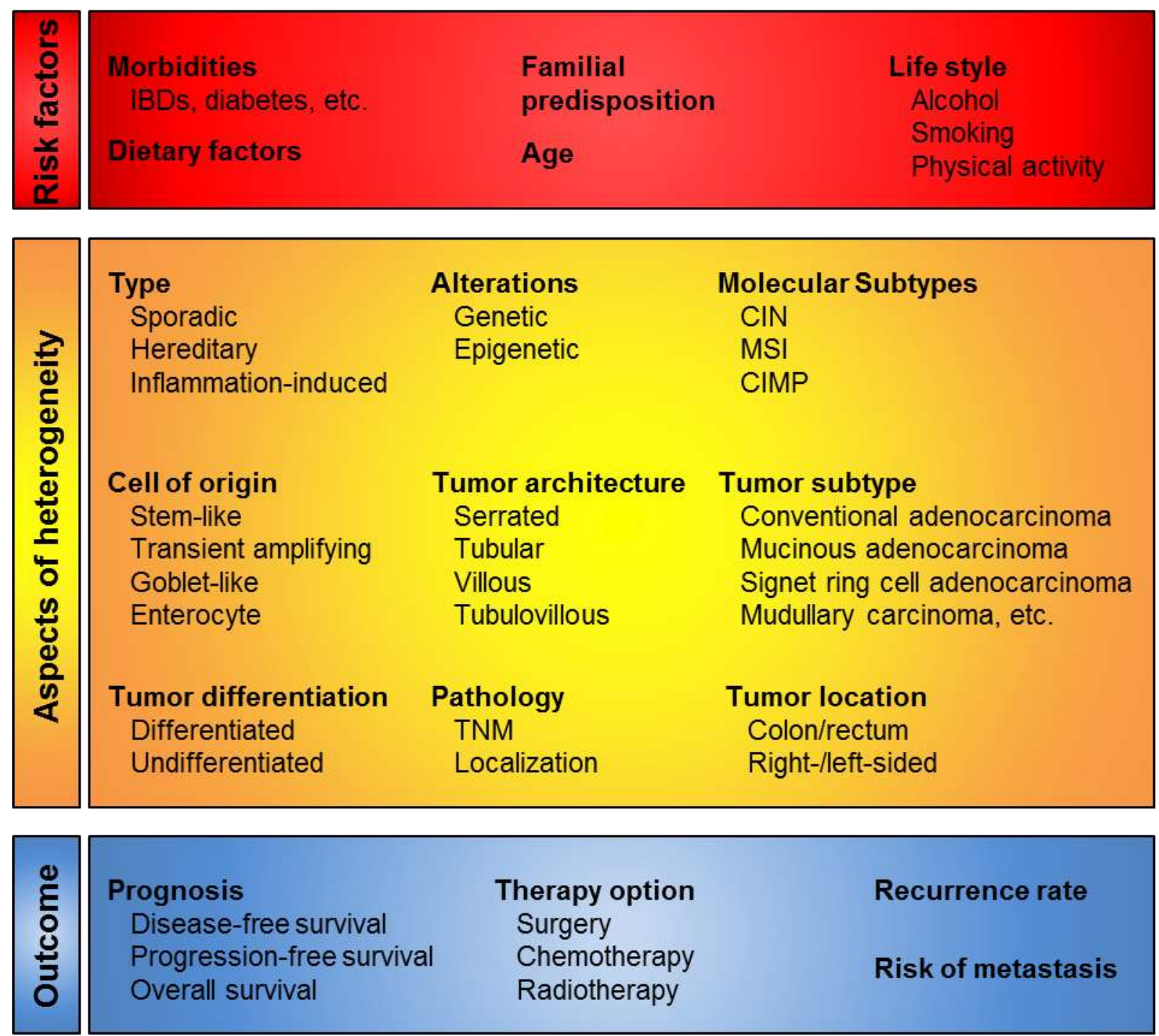

Figure 7: The heterogeneity of colorectal cancer. Several risk factors are associated with colorectal cancer. Once an individual is affected, there are distinct CRC subclasses and 
categories defining the heterogeneity of this disease. These factors can determine therapy choice, prognosis, recurrence rate, and metastasis risk.

Adenocarcinoma subclasses include among others conventional, mucinous or Signet cell adenocarcinomas. However, the pathological CRC staging does not only depend on the primary tumor but also on the presence of nodal and distant metastases. Besides the TNM staging, the tumor site can be a crucial determinant of the characteristics of the cancer lesion. All these factors contribute to the diversity of colorectal cancers and can potentially determine the outcome for the patient, especially recurrence and metastasis. Moreover, the type of therapy is determined based on tumor characteristics and spread.

\subsection{Murine models for ulcerative colitis and colorectal cancer}

Due to its clinical significance, the diverse underlying mechanisms of (inflammation-induced) colorectal cancer need to be elucidated. For research purposes a number of experimental animal models have been generated which closely resemble the human situation of inflammatory bowel disease and intestinal tumorigenesis. For instance, there are xenograft and allograft transplantations, genetic and chemically induced murine models (Robertis et al., 2011).

One commonly selected approach is xenograft in which human cancer cells are injected subcutaneously into immunodeficient mice which allows easy monitoring of tumor growth in vivo (Garofalo et al., 1993). In contrast, in allograft transplantations, also known as syngeneic models, murine cells are injected into mice. Thus, the same species is used in this approach (Voskoglou-Nomikos et al., 2003). Orthotopic transplantation, e.g. injection of human cancer cells into the cecum or rectum of mice, allows the interaction between the tumor and the microenvironment (Hoffman, 1999).

One frequently applied genetic approach is to drive tumorigenesis in the intestine by inducing an APC mutation. Two commonly used models are APC multiple intestinal neoplasia (APC ${ }^{\mathrm{min}}$ ) 
and $\mathrm{APC}^{1638 \mathrm{~N}}$ mice. Both models possess an APC truncation mutation resulting in a nonfunctional APC protein which closely resembles the human situation, e.g. FAP (Fodde and Smits, 2001). In contrast to APC ${ }^{\text {min }}$, where animals primarily display adenomas in the small intestine, the APC ${ }^{1638 \mathrm{~N}}$ mutant mouse model develops fewer tumors in the small intestine and more colorectal tumors. In addition, tumors in APC ${ }^{1638 \mathrm{~N}}$ mice are characterized by frequent progression to carcinomas and occasional metastasis (Fodde et al., 1994; Robanus-Maandag et al., 2010; Taketo and Edelmann, 2009).

To investigate the function of a distinct gene in $\mathrm{CRC}$ formation, it can be conditionally deleted by making use of the Cre/loxP system. Cre ${ }^{\text {ERT2 }}$ mice specifically express a Cre-recombinase as a fusion protein with a mutated Tamoxifen-inducible estrogen receptor ligand binding domain (ERT2). Upon the application of Tamoxifen the Cre-recombinase gets activated and can mediate the excision of a gene segment flanked by loxP-sites frequently resulting in a frameshift and therefore a non-functional gene (Feil et al., 2009). To ensure tissue specificity, the Cre-recombinase is usually expressed under the control of a tissue-specific promoter. For instance, the Villin promoter is mainly expressed in the small intestine and to a lesser extent in the large intestine (el Marjou et al., 2004) while the Caudal-Type Homeobox-2 (CDX2) promoter activity is restricted to the distal ileal, cecal, colonic and rectal epithelium (Feng et al., 2013). In addition to genetic modifications, colorectal tumor formation can be triggered by the induction of colitis. Dextran sulfate sodium (DSS) is a chemical agent administered via the drinking water to induce colitis (Okayasu et al., 1990). Even though the exact underlying mechanisms remain unknown, it is widely accepted that DSS interferes with the epithelial barrier function and has a direct toxic effect on the epithelium. As a result, the mucosal permeability is increased allowing the entry of DSS, luminal antigens and intestinal microorganisms into the mucosa. Consistently, expression levels of proinflammatory cytokines are increased, tight junction protein levels perturbed, apoptosis rates elevated (Perse and Cerar, 2012), finally resulting in intestinal inflammation. 


\subsection{Ubiquitination and cancer}

Taken together, colorectal cancer reflects a highly complex and heterogeneous disorder with numerous associated risk factors and underlying (epi-)genetic alterations. Intensive investigations, especially using suitable animal models, are required to obtain further insights into CRC and its key players and, most importantly, to investigate new therapeutic strategies. As explained before, the ubiquitination status of the histone H2B was described as a predictive marker for tumor grade and stage (Qi et al., 2004). In addition, overexpression of USP22, a factor responsible for H2B deubiquitination, correlates with increased tumor burden. These examples indicate the relevance of maintaining the homeostasis of ubiquitin levels. In contrast to other PTMs, ubiquitination is characterized by conjugating its substrate with a relatively large (8.5 kDa) polypeptide molecule as a result of a three-step process (Figure 8). Generally, ubiquitin is activated in an ATP-dependent manner by the enzyme E1 and conjugated by E2. Subsequently it is covalently linked to a lysine residue of a substrate by an E3 ligase. Ubiquitination can predispose a substrate for proteasomal degradation, however, it is also involved in the regulation of enzyme activity, protein trafficking and the assembly of signaling complexes (Pickart, 2001).

Importantly, this post-translational modification can be reversed by deubiquitinating enzymes which are therefore, together with E3 ligases, responsible for maintaining the homeostasis of cellular ubiquitin levels (Sowa et al., 2009). In humans, approximately 95 DUBs divided into five classes were discovered: JAB1/MPN/MOV34-Metalloprotease (JAMM) domain proteins, Machado-Joseph Domain (Josephin domain)-containing proteins (MJD), Ubiquitin C-Terminal Hydrolases (UCHs), Ubiquitin-Specific Proteases (USPs), and Otubain/Ovarian Tumor Domain-Containing Proteins (OTUs) (Nijman et al., 2005). Interestingly, various DUBs were described to influence gene expression by deubiquitinating histones, transcription factors or their interaction partners. In general, the removal of ubiquitin molecules stabilizes the substrate 
by potentially preventing its degradation. Consequently, if tumor suppressors or oncogenes are affected by DUBs, the risk of developing malignancies can be modulated (McClurg and Robson, 2015).

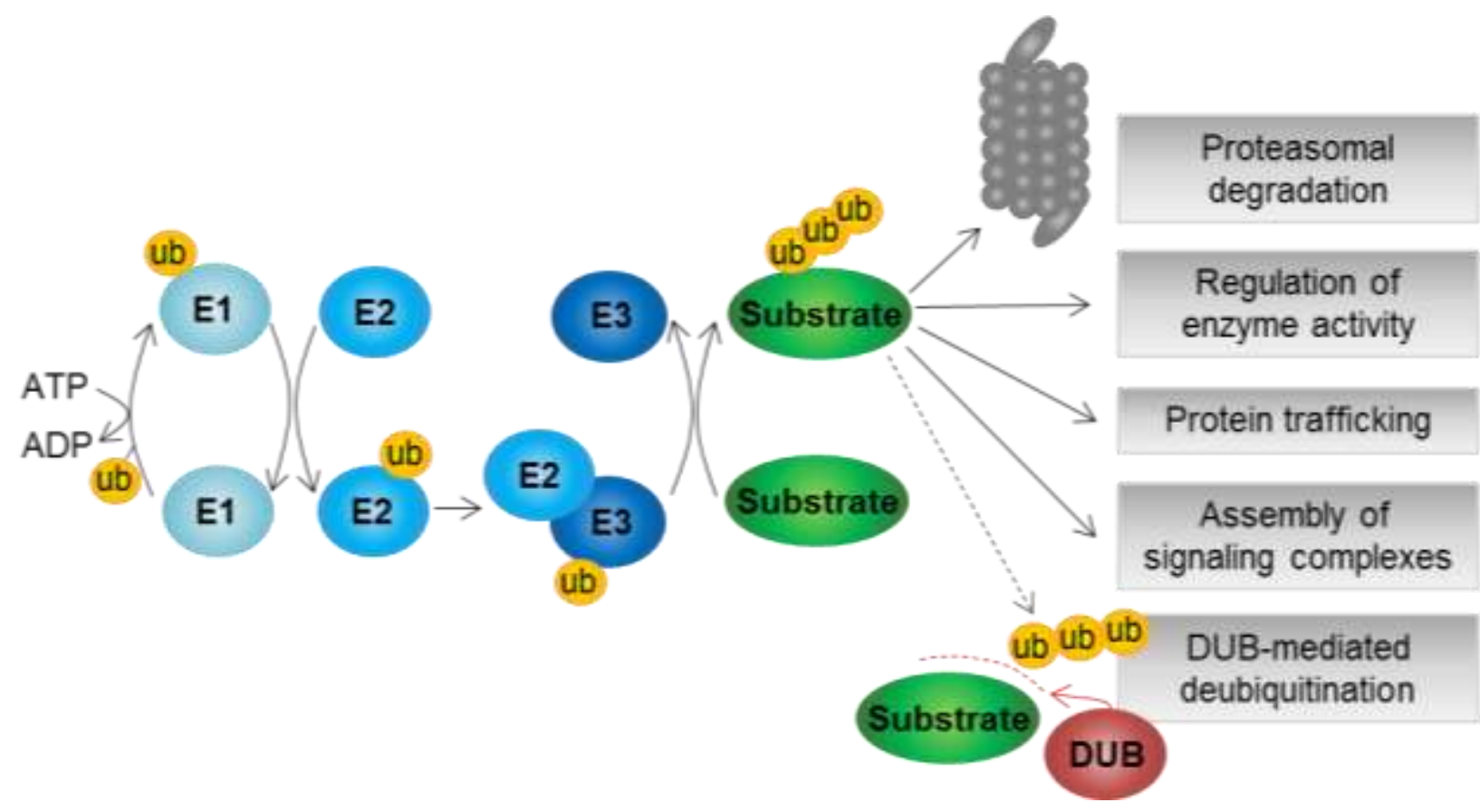

Figure 8: The E1-E2-E3 process of ubiquitination and its consequences. Ubiquitin is conjugated to a substrate in an E1-E2-E3 cascade in which ubiquitin is initially activated by the enzyme E1 in an ATP-dependent manner. Subsequently, ubiquitin is conjugated by E2 and attached to a substrate by an E3 ligase. This mark can result in the proteasomal degradation of the substrate, however, it can also play a role in regulating enzyme activity, protein trafficking and the assembly of signaling complexes. Deubiquitinating enzymes can remove the ubiquitin molecules and thereby stabilize the substrate by potentially preventing its degradation.

To interfere with these mechanisms, it is either possible to prevent the degradation of ubiquitinated molecules, e.g. tumor suppressors, by targeting the proteasome activity or to inhibit the stabilization of oncoproteins by directly inhibiting DUBs. For instance, bortezomib was a FDA-approved drug to target the proteasome multiple myeloma and mantle cell lymphoma patients. However, similar to further proteasome inhibitors, bortezomib was associated with severe side effects since not only cancer cells were targeted by this drug (Liu et al., 2015a; Richardson et al., 2003). In contrast, inhibiting specific DUBs to prevent the stabilization of an oncogenic factor is a promising approach with less off-target effects to be 
expected (Liu et al., 2015a). For instance, the Ubiquitin-Specific Protease 7 was reported to be overexpressed in several cancer types and to interfere with the activity of tumor suppressors such as TP53 by stabilizing MDM2 (Cummins et al., 2004; Li et al., 2004). It was successfully shown cancer cells in vitro that selective USP7 inhibitors were able to exert anti-tumor effects by activating TP53 levels (Colland et al., 2009; Fan et al., 2013; Reverdy et al., 2012). Similarly, inhibiting USP1 and USP14 which are frequently overexpressed in cancer as well, showed promising effects in in vitro approaches and in xenograft models (Dexheimer et al., 2010; Tian et al., 2014). In summary, deregulation of the ubiquitination/deubiquitination axis is associated with various human malignancies by frequently destabilizing tumor suppressors or stabilizing oncogenic factors. The inhibition of the proteasome or DUBs, such as USP1, 7 and 14, reflect promising therapeutic strategies.

\subsection{USP22 as a crucial player of CRC}

Due to its overexpression in cancer and its function as a DUB, the ubiquitin hydrolase UbiquitinSpecific Protease 22 represents a promising research subject. USP22 was described to be a member of the SAGA (Spt-Ada-Gcn5 Acetyltransferase) transcriptional cofactor complex (Zhang et al., 2008b). Generally, the 1.8-MDa SAGA complex was implicated in various mechanisms including chromatin remodeling and transcriptional regulation. These functions are exerted by its structural modules which are, for instance, involved in histone acetylation and deubiquitination (Baker and Grant, 2007; Gurskii et al., 2013). The relevance of SAGA in developmental processes was demonstrated when mutating or depleting Gcn5, Ada2 and Ada3, components of the histone deacetylase (HAT) module within the SAGA complex, induced lethality in Drosophila larvae (Carre et al., 2005; Qi et al., 2004). The ubiquitin hydrolase USP22 is, together with Ataxin 7 Like 3 (ATXN7L3) and Enhancer of Yellow 2 Homolog (ENY2), a component of the deubiquitinating module (DUBm) within this complex. 
Within this complex USP22 removes ubiquitin from the core histones $\mathrm{H} 2 \mathrm{~B}$ and $\mathrm{H} 2 \mathrm{~A}$. While H2B monoubiquitination correlates to active gene expression, the monoubiquitination of $\mathrm{H} 2 \mathrm{~A}$ was associated to gene silencing (Zhang et al., 2008b; Zhang et al., 2008a). Like its yeast orthologue Ubp8, USP22 deubiquitination activity requires its USP domain (Samara et al., 2010). Recently, it has been demonstrated that several functions of USP22 can be taken over by its homologs USP27X and USP51. For instance, USP22, USP27X and USP51 compete for interaction with ATXN7L3 and ENY2 in order to regulate H2Bub1 levels (Atanassov et al., 2016). In addition, USP22 deubiquitinates several non-histone substrates and thereby stabilizes them by preventing their proteasomal degradation. For instance, a protein stabilized by USP22 is Telomeric Repeat Factor 1 (TRF1) which binds to telomere ends and thereby regulates telomere length. When USP22 was depleted in vitro, TRF1 levels were reduced and the number of telomere damage-associated foci increased (Atanassov et al., 2009). Moreover, the fact that complete loss of Usp22 resulted in early embryonic lethality in mice suggested the significance of Usp22 in developmental processes (Lin et al., 2012). In addition, Usp22 expression was shown to be essential for pluripotent stem cells to differentiate into all three germ layers (Sussman et al., 2013).

Glinsky (2005) defined USP22 as a member of the so-called 11-gene "death-from-cancer" gene expression signature which is characterized by a "stem cell-like" expression profile and was shown to be correlated with high recurrence rates, metastatic dissemination and poor survival rates (Glinsky, 2005; Wicha et al., 2006). After this finding, many further studies confirmed its relevance in cancer as recently reviewed (Melo-Cardenas et al., 2016). These reports were based on microarray data or immunohistochemical analyses using patient material and could reveal that increased USP22 abundance was correlated with poor prognosis (Ao et al., 2014; Ding et al., 2014; Hu et al., 2012; Lin et al., 2012; Liu et al., 2010; Liu et al., 2012; Xiao et al., 2015). Moreover, it has been reported that increased USP22 expression was, among others, correlated with gastric carcinoma (Yang et al., 2011), pancreatic (Ning et al., 2014b; Ning et 
al., 2014a) and colorectal cancer (Ao et al., 2015; Liu et al., 2010; Liu et al., 2011). Consistently, reduced levels of USP22 resulted in the accumulation of cancer cells in the G1 phase in vitro (Lin et al., 2015; Lv et al., 2011; Zhang et al., 2008a). Importantly, various key players involved in the development and progression of human malignancies are affected directly or indirectly by USP22. It was demonstrated that USP22 stabilizes Sirtuin 1 (SIRT1) which results in reduced transcription levels of the tumor suppressor TP53 (Li et al., 2014a; Lin et al., 2012). Moreover, in non-small cell lung cancer, USP22 interacts with MDM4, a protein highly similar to MDM2, leading to decreased TP53 activity and, therefore, elevated tumorigenesis (Ding et al., 2014). Besides destabilizing tumor suppressors, USP22 activity was shown to be essential for the transcription of target genes of the oncoprotein c-MYC. Accordingly, USP22 is required for C-MYC-mediated cell transformation (Zhang et al., 2008b). Furthermore, USP22 deubiquitinates and stabilizes Nuclear Factor of Activated T cells 2 (NFATc2) which regulates transcription of interleukin 2, a mediator of T effector cell activation (Gao et al., 2014). This finding implicated a role of USP22 in controlling the immune response and therefore potentially in inflammatory processes. In addition, deubiquitinating $\mathrm{H} 2 \mathrm{~B}$, and thus decreasing levels of the potential tumor suppressive mark H2Bub1, is a further mechanism implying the relevance of USP22 during tumorigenesis. Based on these studies and as discussed by Melo-Cardenas and colleagues USP22 represents an attractive therapeutic target (Melo-Cardenas et al., 2016). Similar to USP7, an inhibitor could be generated interfering with USP22 activity and therefore preventing the stabilization of several oncoproteins.

While the involvement of USP22 in cancer has been demonstrated several times, the function of USP22 in tumorigenesis in vivo using genetic mouse tumor models with a tissue-specific knockout of Usp22 has not been investigated so far. Thus, the exact mechanisms by which USP22 promotes tumorigenesis remain to be elucidated. 


\subsection{Implications of USP22 in intestinal cell differentiation (preliminary data)}

Prior to the current project, we investigated the role of USP22 in organ maintenance using Usp22 hypomorphic mice (Kosinsky, 2013). In this M.Sc. thesis a preliminary characterization of mice with heterozygous (Usp22 lacz/wt) and homozygous (Usp22laczlacz) reduction of Usp22 compared to wild type (Usp22 $2^{\text {thtwt }}$ ) animals was performed. Embryonic stem cells (Usp22 ${ }^{\text {tm1a(KOMP)Wtsi }}$ C57BI6) were obtained from the University of California-Davis Knockout Mouse Project Repository and mice were generated in cooperation with Prof. Dr. Ahmed Mansouri (Department of Molecular Cell Biology, Max-Planck Institute for Biophysical Chemistry, Göttingen, Germany). Based on the "knockout first" strategy these mice had a LacZ cassette and a neomycin resistance cassette inserted into the first intron of the Usp22 gene (Figure 9A). Due to the presence of an Engrailed2 splice acceptor site (EN2-SA-IRES) the LacZ reporter gene was expressed under the control of the endogenous Usp22 promoter and enabled the visualization of Usp22 gene activity. A reduction of Usp22 expression was achieved by stop codons and polyadenylation sequences downstream of the LacZ and neomycin resistance genes, while leaving the Usp22 gene intact.

Notably, Usp22 laczlacz animals were characterized by reduced body size and weight. Analyses were restricted to the small intestine in which we could not detect differences regarding proliferation and senescence rates. However, slightly increased apoptosis levels were shown. Moreover, the cellular composition of the small intestine was evaluated. In situ hybridization and immunohistochemical stainings were performed in order to visualize the different cell populations of the SI, i.e. Olfm4-positive stem cells, Goblet, enteroendocrine and Paneth cells. Interestingly, the number of stem cells was slightly increased in the small intestines of Usp22 lacZlacz animals. Consequently, we aimed to determine whether this alteration had an effect on the respective differentiated cell populations. Immunohistochemistry was performed using specific markers for Goblet (Mucin 2; MUC2), enteroendocrine (Chromogranin A; CGA) and Paneth (Lysozyme; LYZ) cells. Interestingly, reduced levels of Usp22 resulted in a nearly 
twofold elevated abundance of MUC2-positive Goblet cells in villi (Figure 9B). Moreover, approximately $45-50 \%$ more enteroendocrine cells were counted in the crypts and villi in these mice. We also found an increased frequency of Paneth cells in Usp22 laczllacz mice. Together, these findings suggested that USP22 is required for epithelial cell differentiation in villi and crypts. In addition, mouse embryonic fibroblasts (MEFs) were isolated and partially characterized. While apoptosis levels were slightly increased upon Usp22 loss, the cell cycle profile was not affected upon Usp22 loss (Kosinsky, 2013).

For the current project we have generated bigger cohorts of Usp22-hypomorphic mice in order to analyze the effect of hypomorphic expression of Usp22 in more detail. In addition, for a higher validity and significance of data, not only the number of experimental animals was increased, but also some approaches presented in the M.Sc. thesis were repeated. Several results of this Ph.D. thesis supplemented by data from the M.Sc. thesis have recently been published (Kosinsky et al., 2015). 


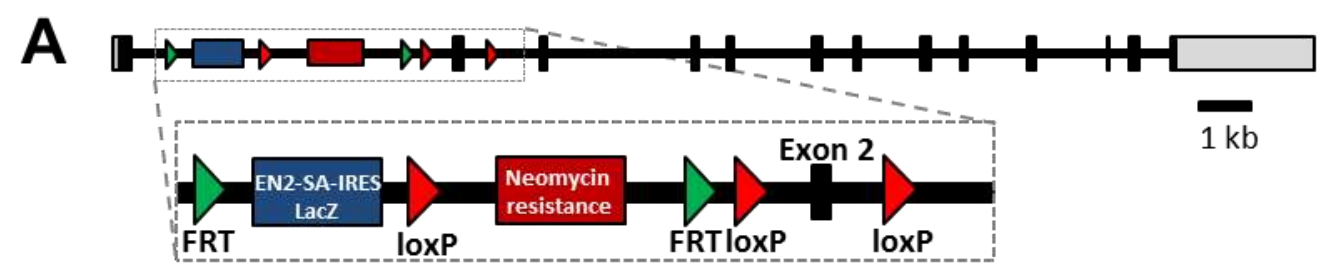

B

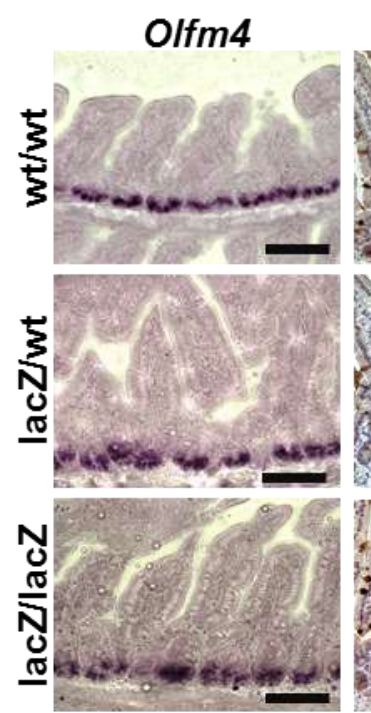

MUC2

CGA

LYZ
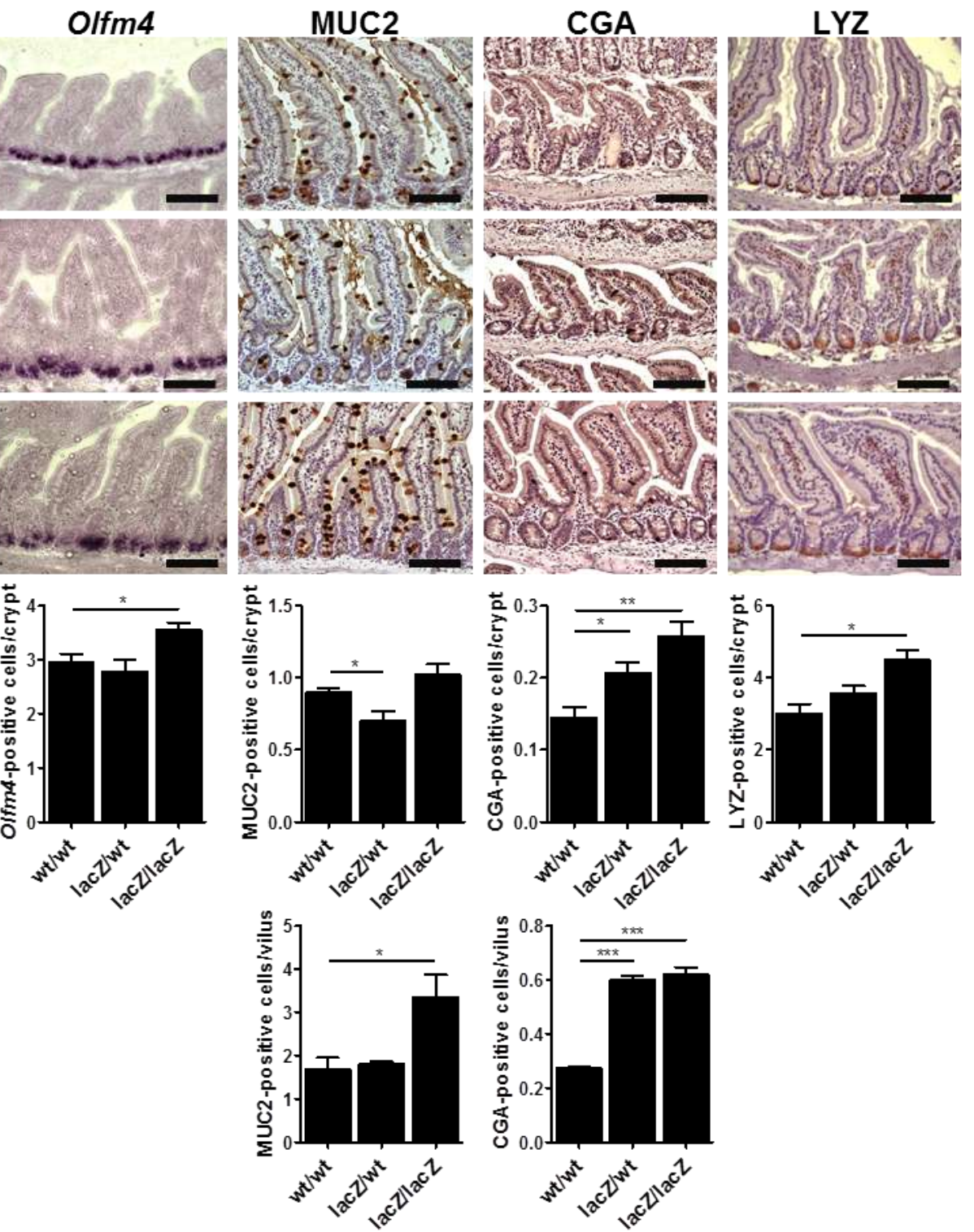

Figure 9: Reduced Usp22 levels lead to a differentiation shift in the small intestine. (A) The LacZ reporter gene is expressed downstream of the Usp22 promoter. Selection of embryonic stem cells was based on a neomycin-resistance cassette. Stop codons and poly-A sites mediated the reduced Usp22 expression. (B) In situ hybridization for the stem cell marker Olfm4 revealed an increased presence of stem cells in the small intestines of Usp22hypomorphic Usp22 lacz/lacz mice. These mice were characterized by an increased abundancy of differentiated cells as shown by immunohistochemistry using specific markers, i.e. Goblet (MUC2), enteroendocrine (CGA) and Paneth (LYZ) cells (Kosinsky et al., 2015). Scale bar: $100 \mu \mathrm{m}$. 


\subsection{Objectives of this study}

To contribute to the understanding of the molecular features underlying the complex and heterogeneous nature of CRC we aimed to elucidate the function of USP22 in tumor formation and progression. USP22, an ubiquitin hydrolase epigenetically modifying the histone $\mathrm{H} 2 \mathrm{~B}$, is of particular interest since it appears to be involved in colorectal tumorigenesis. Notably, cancerous diseases characterized by a USP22 overexpression were correlated with poor prognosis and metastatic dissemination. Even though some USP22 targets were identified so far and several studies with USP22-related in vitro approaches have been published, the physiological significance and the role of USP22 in colorectal cancer remain largely unclear. This clearly demonstrates the need to investigate the role of USP22 under physiological conditions and during colorectal tumorigenesis including the underlying alterations in more detail both in vitro and in vivo with the help of a genetic animal model.

For this purpose, we aimed to follow a dual approach by analyzing the consequences of a global reduction and an intestine-specific Usp22 deletion. In the Usp22-hypomorphic mouse model which was partially described in the aforementioned Master's thesis, a global Usp22 reduction was achieved. Here, we intended to focus on the expression patterns of Usp22 during different developmental stages of mouse embryos. Furthermore, by isolating mouse embryonic fibroblasts, we planned to investigate signaling pathways Usp22 is involved in via microarray analysis. In addition to the animals with the global reduction of Usp22 expression levels, we generated mice with an intestine-specific deletion of Usp22 combined with an APC mutation. Using these mice we sought to investigate the function of USP22 in intestinal cancer formation and progression in models for sporadic and inflammation-induced colorectal cancer. Based on the literature, we hypothesized that Usp22 loss would result in decreased tumor burden. To investigate the molecular structures underlying the phenotype observed in our mice, we planned to investigate human colorectal cell lines characteristics upon the loss of USP22. Besides analyses of proliferation patterns, morphology and migration potential, we sought to 
identify the consequence of USP22 loss in human CRC cells on the global gene expression profile. Finally, it was our aim to identify therapeutic mechanisms to target colorectal cancer cells characterized by altered USP22 expression in vivo. Together, in the current project we sought to investigate the function of USP22 under physiological conditions and during CRC as well as to determine the potential of USP22 as a diagnostic marker and therapeutic target. 


\section{MAterials and Methods}

\subsection{Materials}

\subsubsection{Technical devices}

Table 1: Technical devices.

\begin{tabular}{|c|c|}
\hline Equipment & Company \\
\hline 2100 Bioanalyzer & Agilent Technology, Santa Clara, USA \\
\hline Balance $440-35 \mathrm{~N}$ & Kern \& Sohn GmbH, Balingen, Germany \\
\hline Biological Safety Cabinet “Safe 2020” & Thermo Fisher Scientific, Waltham, USA \\
\hline Bioruptor $^{\circledR}$ Plus sonication device & Diagenode, Liège, Belgium \\
\hline BioView UV-transilluminator UXDT-20SM-8R & BioStep, Jahnsdorf, Germany \\
\hline Celigo ${ }^{\circledR}$ S Cell Imaging Cytometer & Nexcelom Bioscience LLC, Lawrence, USA \\
\hline Centrifuge Heraeus Fresco21 & Thermo Fisher Scientific, Waltham, USA \\
\hline Centrifuge $5417 \mathrm{C}$ & Eppendorf AG, Hamburg, Germany \\
\hline Centrifuge $5417 R$ & Eppendorf AG, Hamburg, Germany \\
\hline Centrifuge Heraeus $^{\mathrm{TM}}$ Megafuge $^{\mathrm{TM}} 8 \mathrm{R}$ & Thermo Fisher Scientific, Waltham, USA \\
\hline Centrifuge Mini Star silverline & VWR, Radnor, USA \\
\hline CFX Connect Real-Time System & Bio-Rad Laboratories, Hercules, USA \\
\hline $\begin{array}{l}\text { CFX96 TM Optical Reaction Module for Real- } \\
\text { Time PCR }\end{array}$ & Bio-Rad Laboratories, Hercules, USA \\
\hline ChemiDoc $^{T M}$ MP System & Bio-Rad Laboratories, Hercules, USA \\
\hline CoolLED pE100 & CoolLED Ldt., Andover, UK \\
\hline Cryotome CM1850 & $\begin{array}{l}\text { Leica Mikrosysteme Vertrieb GmbH, } \\
\text { Wetzlar, Germany }\end{array}$ \\
\hline Digital Caliper (0-150 mm) & Zisaline GmbH, Bärnau, Germany \\
\hline
\end{tabular}




\begin{tabular}{|c|c|}
\hline DLReady ${ }^{\mathrm{TM}}$ Centro LB 960 Luminometer & $\begin{array}{l}\text { Berthold Technologies GmbH + Co. KG, } \\
\text { Stadthagen, Germany }\end{array}$ \\
\hline DS-11+ spectrophotometer & DeNovix, Inc., Wilmington, USA \\
\hline Freezer $\left(-150^{\circ} \mathrm{C}\right) \mathrm{MDF}-\mathrm{C} 2156 \mathrm{VAN}-\mathrm{PE}$ & $\begin{array}{l}\text { Ewald Innovationstechnik GmbH, Bad } \\
\text { Nenndorf, Germany }\end{array}$ \\
\hline Freezer $\left(-20^{\circ} \mathrm{C}\right)$ & Liebherr GmbH, Biberach, Germany \\
\hline Freezer $\left(-80^{\circ} \mathrm{C}\right)$ "New Brunswick ${ }^{\mathrm{TM}}$ Innova ${ }^{\circledR ”}$ & $\begin{array}{l}\text { Eppendorf GmbH, Wesseling-Berzdorf, } \\
\text { Germany }\end{array}$ \\
\hline Glass Hellendahl cuvettes for histology & $\begin{array}{l}\text { Omnilab-Laborzentrum GmbH \& Co. KG, } \\
\text { Bremen, Germany }\end{array}$ \\
\hline Glass slide racks with handle for histology & $\begin{array}{l}\text { Omnilab-Laborzentrum GmbH \& Co. KG, } \\
\text { Bremen, Germany }\end{array}$ \\
\hline Glass staining dish with cover for histology & $\begin{array}{l}\text { Omnilab-Laborzentrum GmbH \& Co. KG, } \\
\text { Bremen, Germany }\end{array}$ \\
\hline Heating block Thermo Mixer C & Eppendorf AG, Hamburg, Germany \\
\hline Heating plate for slides & Thermo Fisher Scientific, Waltham, USA \\
\hline $\begin{array}{l}\text { Horizon }{ }^{\circledR} 58 \text { Agarose Gel Electrophoresis } \\
\text { Chamber }\end{array}$ & Life Technologies ${ }^{\mathrm{TM}}$, Gaithersburg, USA \\
\hline Humidified chamber for histology & Weckert Labortechnik, Kitzingen, Germany \\
\hline Ice-machine B100 & Ziegra, Isernhagen, Germany \\
\hline Incubator $\left(\mathrm{CO}_{2}\right)$ for cell culture, Hera Cell $150 \mathrm{i}$ & Thermo Fisher Scientific, Waltham, USA \\
\hline Incubator for histology & $\begin{array}{l}\text { Memmert GmbH \& Co. KG, Schwabach, } \\
\text { Germany }\end{array}$ \\
\hline Inverted Routine Microscope "Eclipse TS100" & Nikon Corporation, Tokyo, Japan \\
\hline Isotemp ${ }^{\circledR}$ water bath & Thermo Fisher Scientific, Waltham, USA \\
\hline Liquid nitrogen tank LS4800 & Worthington Industries, Theodore, USA \\
\hline Magnetic stirrer "IKA ${ }^{\circledR} R C T$-basic" & $\begin{array}{l}\text { IKA }{ }^{\circledR} \text {-Werke GmbH \& Co. KG, Staufen im } \\
\text { Breisgau, Germany }\end{array}$ \\
\hline Microcentrifuge C1413-VWR230 & VWR, Radnor, USA \\
\hline
\end{tabular}




\begin{tabular}{|c|c|}
\hline Microm EC350 embedding station & Thermo Fisher Scientific, Waltham, USA \\
\hline Microscope Axio Scope.A1 with AxioCam MRc & $\begin{array}{l}\text { Carl Zeiss Microlmaging GmbH, Göttingen, } \\
\text { Germany }\end{array}$ \\
\hline Microscope Axiovert 100 & $\begin{array}{l}\text { Carl Zeiss Microlmaging GmbH, Göttingen, } \\
\text { Germany }\end{array}$ \\
\hline Microscope camera UI-1240ML & iDS, Obersulm, Germany \\
\hline Microtome Leica RM2235 & Leica Biosystems, Nussloch, Germany \\
\hline Microwave R937 & $\begin{array}{l}\text { Sharp Electronics Europe GmbH, Hamburg, } \\
\text { Germany }\end{array}$ \\
\hline Mini Trans-Blot ${ }^{\circledR}$ Cell & Bio-Rad Laboratories, Hercules, USA \\
\hline Mini-PROTEAN Tetra Cell & Bio-Rad Laboratories, Hercules, USA \\
\hline Mr. Frosty ${ }^{\circledR}$ Cryo Freezing Container & Thermo Fisher Scientific, Waltham, USA \\
\hline NanoDrop ${ }^{\circledR}$ ND-100 spectrophotometer & PeqLab, Erlangen, Germany \\
\hline Neubauer counting chamber, Improved & Brand GmbH \& Co. KG, Wertheim, Germany \\
\hline Paraffin oven & Thermo Fisher Scientific, Waltham, USA \\
\hline PCR machine $\mathrm{T} 100^{\mathrm{TM}}$ Thermal cycler & Bio-Rad Laboratories, Hercules, USA \\
\hline Personal Computer OPTIPLEX 7020 & Dell, Round Rock, USA \\
\hline pH-meter “WTW-720" InoLab ${ }^{\circledR}$ Series & WTW GmbH, Weilheim, Germany \\
\hline Pipette Aid ${ }^{\circledR}$ Portable XP & Drummond Scientific Co., Broomall, USA \\
\hline Pipettes "Research" Series & Eppendorf AG, Hamburg, Germany \\
\hline PowerLyzer ${ }^{\mathrm{TM}} 24$ Homogenizer & $\begin{array}{l}\text { MO BIO Laboratories/QIAGEN, Carlsbad, } \\
\text { USA }\end{array}$ \\
\hline PowerPac ${ }^{\top M}$ Basic Power Supply & Bio-Rad Laboratories, Hercules, USA \\
\hline PowerPac ${ }^{T M}$ HC Power Supply & Bio-Rad Laboratories, Hercules, USA \\
\hline Precision balance TE124S & Sartorius AG, Göttingen, Germany \\
\hline $\begin{array}{l}\text { Protein electrophoresis Mini-PROTEAN }{ }^{\circledR} \text { Tetra } \\
\text { Handcast Systems }\end{array}$ & Bio-Rad Laboratories, Hercules, USA \\
\hline
\end{tabular}




\begin{tabular}{|l|l|}
\hline Qubit ${ }^{\circledR} 2.0$ Fluorometer & Invitrogen GmbH, Karlsruhe, Germany \\
\hline Refrigerator $\left(4^{\circ} \mathrm{C}\right)$ & Liebherr GmbH, Biberach, Germany \\
\hline Roller mixer & A. Hartenstein GmbH,Würzburg, Germany \\
\hline Scanner Epson V700 Photo & Seiko Epson, Suwa, Japan \\
\hline Shaker “Rocky" & $\begin{array}{l}\text { Schütt Labortechnik GmbH, Göttingen, } \\
\text { Germany }\end{array}$ \\
\hline Stainless feeding tubes (12 ga, $76 \mathrm{~mm})$ & $\begin{array}{l}\text { Instech Laboratories, Inc., Plymouth } \\
\text { Meeting, USA }\end{array}$ \\
\hline Test tube rotator & $\begin{array}{l}\text { Schütt Labortechnik GmbH, Göttingen, } \\
\text { Germany }\end{array}$ \\
\hline Ultra pure water system “Aquintus” & MembraPure GmbH, Hennigsdorf, Germany \\
\hline Vacuum pump BVC control & $\begin{array}{l}\text { Vacuubrand GmbH + Co KG, Wertheim, } \\
\text { Germany }\end{array}$ \\
\hline Vortex Genie 2 & $\begin{array}{l}\text { Electro Scientific Industries, Inc., Portland, } \\
\text { USA }\end{array}$ \\
\hline Zwick device (145 660 Z020/TND) & Zwick GmbH \& Co. KG, Ulm, Germany \\
\hline
\end{tabular}

\subsubsection{Consumables}

Table 2: Consumables.

\begin{tabular}{|l|l|}
\hline Equipment & Company \\
\hline $\begin{array}{l}\text { 8.0- } \mu \mathrm{m} \text { track-etched membrane cell culture } \\
\text { inserts }\end{array}$ & BD Bioscience, Franklin Lakes, USA \\
\hline 96-well Assay plate black & Corning Life sciences, Tewksbury, USA \\
\hline 96-well Multiplate ${ }^{\circledR} \mathrm{PCR}$ plate, white & Bio-Rad Laboratories, Hercules, USA \\
\hline $\begin{array}{l}\text { Amersham } \\
\text { TM } \text { Protran }^{\mathrm{TM}} 0.45 \mu \mathrm{M}\end{array}$ & $\begin{array}{l}\text { GE Healthcare Europe GmbH, München, } \\
\text { Germany }\end{array}$ \\
\hline $\begin{array}{l}\text { CA-Membrane sterile filters }(0.2 \mu \mathrm{m}, 0.45 \\
\mu \mathrm{m})\end{array}$ & Sarstedt AG \& Co., Nümbrecht, Germany \\
\hline
\end{tabular}




\begin{tabular}{|c|c|}
\hline Cell culture dishes $(10 \mathrm{~cm}, 14.5 \mathrm{~cm})$ & $\begin{array}{l}\text { Greiner Bio-One GmbH, Frickenhausen, } \\
\text { Germany }\end{array}$ \\
\hline Cell culture plates (6-well, 12-well, 24-well) & $\begin{array}{l}\text { Greiner Bio-One GmbH, Frickenhausen, } \\
\text { Germany }\end{array}$ \\
\hline Cell scraper $(16 \mathrm{~cm}, 25 \mathrm{~cm})$ & Sarstedt AG \& Co., Nümbrecht, Germany \\
\hline Cellstar PP-tubes (15, 50 ml) & $\begin{array}{l}\text { Greiner Bio-One GmbH, Frickenhausen, } \\
\text { Germany }\end{array}$ \\
\hline Cover slips $(24 \times 40,24 \times 60 \mathrm{~mm})$ & $\begin{array}{l}\text { Carl Roth GmbH \& Co. KG, Karlsruhe, } \\
\text { Germany }\end{array}$ \\
\hline Cryo Tube ${ }^{\mathrm{TM}}$ vials $(1.8 \mathrm{ml})$ & Thermo Fisher Scientific, Waltham, USA \\
\hline Disposable Safety Scalpel & FEATHER Safety Razor Co., Osaka, Japan \\
\hline EDTA 40n flat bottom test tubes & $\begin{array}{l}\text { KABE Labortechnik GmbH, Nümbrecht- } \\
\text { Elsenroth, Germany }\end{array}$ \\
\hline Embedding cassettes, for biopsies & $\begin{array}{l}\text { Carl Roth GmbH \& Co. KG, Karlsruhe, } \\
\text { Germany }\end{array}$ \\
\hline Gel blotting paper (Whatman) & Sartorius AG, Göttingen, Germany \\
\hline Injekt-F Syringes (1 ml) & B. Braun, Melsungen, Germany \\
\hline Low-profile disposable blades 819 & Leica Biosystems, Nussloch, Germany \\
\hline Lysing Matrix Tubes with beads & MP Biomedicals, Eschwege \\
\hline Microscope slides (75x26 mm) & Thermo Scientific, Braunschweig, Germany \\
\hline Multiply PCR Microtube strip $(8 \times 0.2 \mathrm{ml})$ & Sarstedt AG \& Co., Nümbrecht, Germany \\
\hline OptiPlate ${ }^{\mathrm{TM}}-96$ & PerkinEImer, Waltham, USA \\
\hline Parafilm ${ }^{\circledR}$ “M” & Pechiney Plastic Packaging, Chicago, USA \\
\hline PCR Single Cap SoftStrips $0.2 \mathrm{ml}$ & Biozym, Oldendorf, Germany \\
\hline $\begin{array}{l}\text { Peel away cryo embedding molds ( } 12 \times 12 \\
\mathrm{~mm})\end{array}$ & Electron Microscopy Sciences, Hatfield, USA \\
\hline Pipet tips $(0.5-10 \mu \mathrm{l}, 2-200 \mu \mathrm{l}, 1000 \mu \mathrm{l})$ & $\begin{array}{l}\text { Greiner Bio-One GmbH, Frickenhausen, } \\
\text { Germany }\end{array}$ \\
\hline Pipets, serological (5 ml, 10 ml, $25 \mathrm{ml}$ ) & Sarstedt AG \& Co., Nümbrecht, Germany \\
\hline
\end{tabular}




\begin{tabular}{|l|l|}
\hline Pipette filter tips & Sarstedt AG \& Co., Nümbrecht, Germany \\
\hline Reaction tubes $(1.5 \mathrm{ml}, 2 \mathrm{ml})$ & Sarstedt AG \& Co., Nümbrecht, Germany \\
\hline Sealing tape for qPCR plates & Bio-Rad Laboratories, Hercules, USA \\
\hline Soft-Ject $^{\circledR}$ Syringes $(10 \mathrm{ml}, 20 \mathrm{ml}, 50 \mathrm{ml})$ & Henke Sass Wolf GmbH, Tuttlingen \\
\hline SuperFrost ${ }^{\circledR}$ Plus slides $(75 \times 25 \mathrm{~mm})$ & Thermo Scientific, Braunschweig, Germany \\
\hline Syringe canula $(0.6 \times 25 \mathrm{~mm}, 0.3 \times 12 \mathrm{~mm})$ & Dispomed Witt oHG, Gelnhausen, Germany \\
\hline Transfer pipettes $(6 \mathrm{ml})$ & Sarstedt AG \& Co., Nümbrecht, Germany \\
\hline UV-Cuvette micro $(8.5 \mathrm{~mm})$ & Brand GmbH, Wertheim, Germany \\
\hline
\end{tabular}

\subsubsection{Chemicals and reagents}

Table 3: Chemicals and reagents.

\begin{tabular}{|l|l|}
\hline Chemical & Company \\
\hline $0.9 \% \mathrm{NaCl}$ solution & B. Braun, Melsungen, Germany \\
\hline $10 x$ reaction buffer for M-MulV & $\begin{array}{l}\text { New England BioLabs GmbH, Frankfurt am Main, } \\
\text { Germany }\end{array}$ \\
\hline $\begin{array}{l}3,3 \text { '-Diaminobenzidin- } \\
\text { tetrahydrochloride (DAB) }\end{array}$ & Carl Roth GmbH \& Co. KG, Karlsruhe, Germany \\
\hline Acetic acid & Carl Roth GmbH \& Co. KG, Karlsruhe, Germany \\
\hline $\begin{array}{l}\text { Adenosine 5'-triphosphate (ATP) } \\
\text { disodium salt hydrate }\end{array}$ & Sigma-Aldrich Co., St. Louis, USA \\
\hline Agarose & GeneOn GmbH, Ludwigshafen am Rhein, Germany \\
\hline Albumin Fraction V (BSA) & Carl Roth GmbH \& Co. KG, Karlsruhe, Germany \\
\hline Ammonium persulfate $(\mathrm{APS})$ & Carl Roth GmbH \& Co. KG, Karlsruhe, Germany \\
\hline Ammonium sulfate (NH $\left.{ }_{4}\right)_{2} \mathrm{SO}_{4}$ & Carl Roth GmbH \& Co. KG, Karlsruhe, Germany \\
\hline Aprotinin & Carl Roth GmbH \& Co. KG, Karlsruhe, Germany \\
\hline Boric acid & Carl Roth GmbH \& Co. KG, Karlsruhe, Germany \\
\hline
\end{tabular}




\begin{tabular}{|c|c|}
\hline Bromophenol blue & Sigma-Aldrich Co., St. Louis, USA \\
\hline Butella Sunflower Oil & $\begin{array}{l}\text { Brökelmann + Co, Oelmühle GmbH + Co, Hamm, } \\
\text { Germany }\end{array}$ \\
\hline Chloroform & Merck Millipore, Darmstadt, Germany \\
\hline Citric acid monohydrate & Merck Millipore, Darmstadt, Germany \\
\hline Coelenterazine & Promega, Madison, USA \\
\hline Coenzyme A (CoA) & Sigma-Aldrich Co., St. Louis, USA \\
\hline Crystal violet & Merck Millipore, Darmstadt, Germany \\
\hline Deoxycholic acid (DOC) & AppliChem GmbH, Darmstadt, Germany \\
\hline Deoxynucleotides (dNTPs) & Jena Bioscience GmbH, Jena, Germany \\
\hline $\begin{array}{l}\text { Dextrane Sulfate Sodium Salt, reagent } \\
\text { grade }\end{array}$ & MO Biomedicals, LLC, Illkirch, France \\
\hline Diethylpyrocarbonate (DEPC) & Carl Roth GmbH \& Co. KG, Karlsruhe, Germany \\
\hline Dimethyl sulfoxide (DMSO) & Carl Roth GmbH \& Co. KG, Karlsruhe, Germany \\
\hline Dimethylformamide & Carl Roth GmbH \& Co. KG, Karlsruhe, Germany \\
\hline Dipotassium phosphate $\left(\mathrm{K}_{2} \mathrm{HPO}_{4}\right)$ & Carl Roth GmbH \& Co. KG, Karlsruhe, Germany \\
\hline $\begin{array}{l}\text { DL-Dithiothreitol (DTT), BioUltra, } \\
\geq 99.0 \%\end{array}$ & Sigma-Aldrich Co., St. Louis, USA \\
\hline Eosin $Y$ solution, $1 \%$ in water & Carl Roth GmbH \& Co. KG, Karlsruhe, Germany \\
\hline Ethanol & Carl Roth GmbH \& Co. KG, Karlsruhe, Germany \\
\hline $\begin{array}{l}\text { Ethylene diamine tetraacetic acid } \\
\text { (EDTA) }\end{array}$ & Carl Roth GmbH \& Co. KG, Karlsruhe, Germany \\
\hline $\begin{array}{l}\text { Ethylene diamine tetraacetic acid } \\
\left.\text { disodium salt dihydrate ( } \mathrm{Na}_{2} \mathrm{EDTA}\right)\end{array}$ & Carl Roth GmbH \& Co. KG, Karlsruhe, Germany \\
\hline ExtrAvidin-Peroxidase & Sigma-Aldrich Co., St. Louis, USA \\
\hline Ethylene glycol tetraacetic acid (EGTA) & Carl Roth GmbH \& Co. KG, Karlsruhe, Germany \\
\hline $\begin{array}{l}\text { Formaldehyde solution } 35 \% \text {, DAB, for } \\
\text { histology }\end{array}$ & Carl Roth GmbH \& Co. KG, Karlsruhe, Germany \\
\hline
\end{tabular}




\begin{tabular}{|c|c|}
\hline Gene Ruler ${ }^{\mathrm{TM}}$ DNA-Ladder & Fermentas GmbH, St. Leon-Rot, Germany \\
\hline $\begin{array}{l}\text { Glutaraldehyde, 25\% EM Grade } \\
\text { Aqueous }\end{array}$ & Sigma-Aldrich Co., St. Louis, USA \\
\hline Glycerol & Carl Roth GmbH \& Co. KG, Karlsruhe, Germany \\
\hline Glycine & Carl Roth GmbH \& Co. KG, Karlsruhe, Germany \\
\hline GlycylGlycine & Sigma-Aldrich Co., St. Louis, USA \\
\hline Guaiac resin & Carl Roth GmbH \& Co. KG, Karlsruhe, Germany \\
\hline HD Green ${ }^{\circledR}$ DNA stain & Intas Science Imaging GmbH, Göttingen, Germany \\
\hline HEPES & Carl Roth GmbH \& Co. KG, Karlsruhe, Germany \\
\hline Hydrochloric acid $(\mathrm{HCl})$ & Carl Roth GmbH \& Co. KG, Karlsruhe, Germany \\
\hline $\begin{array}{l}\text { Hydrogen peroxide solution }\left(\mathrm{H}_{2} \mathrm{O}_{2}\right) \text {, } \\
30 \%\end{array}$ & Carl Roth GmbH \& Co. KG, Karlsruhe, Germany \\
\hline lodoacetamide & Sigma-Aldrich Co., St. Louis, USA \\
\hline Isopropanol & Carl Roth GmbH \& Co. KG, Karlsruhe, Germany \\
\hline Leupeptin & Carl Roth GmbH \& Co. KG, Karlsruhe, Germany \\
\hline Lipofectamine ${ }^{\circledR} 2000$ & Invitrogen $\mathrm{GmbH}$, Karlsruhe , Germany \\
\hline Lipofectamine ${ }^{\circledR}$ RNAiMAX & Invitrogen $\mathrm{GmbH}$, Karlsruhe , Germany \\
\hline Magnesium chloride $\left(\mathrm{MgCl}_{2}\right)$ & Carl Roth GmbH \& Co. KG, Karlsruhe, Germany \\
\hline Magnesium sulfate $\left(\mathrm{MgSO}_{4}\right)$ & Carl Roth GmbH \& Co. KG, Karlsruhe, Germany \\
\hline Mayer's haematoxylin solution & Merck Millipore, Darmstadt, Germany \\
\hline Methanol & Carl Roth GmbH \& Co. KG, Karlsruhe, Germany \\
\hline N-ethylmaleimide (NEM) & Sigma-Aldrich Co., St. Louis, USA \\
\hline Nonidet $^{\circledast}$ P 40 Substitute (NP-40) & Sigma-Aldrich Co., St. Louis, USA \\
\hline $\begin{array}{l}\text { Nuclear fast red-aluminum sulfate } \\
\text { solution } 0.1 \%\end{array}$ & Carl Roth GmbH \& Co. KG, Karlsruhe, Germany \\
\hline $\begin{array}{l}\text { PageRuler }{ }^{\top \mathrm{M}} \text { Plus Prestained Protein } \\
\text { Ladder }\end{array}$ & Fermentas GmbH, St. Leon-Rot, Germany \\
\hline
\end{tabular}




\begin{tabular}{|c|c|}
\hline Paraffin Rotiplast & Carl Roth GmbH \& Co. KG, Karlsruhe, Germany \\
\hline Paraformaldehyde (PFA) & Carl Roth GmbH \& Co. KG, Karlsruhe, Germany \\
\hline Passive lysis buffer $(5 x)$ & Promega, Madison, USA \\
\hline PBS tablets & GIBCO $^{\circledR}$, Invitrogen $\mathrm{GmbH}$, Darmstadt, Germany \\
\hline Pefabloc SC Protease Inhibitor & Carl Roth GmbH \& Co. KG, Karlsruhe, Germany \\
\hline $\mathrm{pH}$ solutions $(\mathrm{pH} \mathrm{4.01,7.01,10.01)}$ & Carl Roth GmbH \& Co. KG, Karlsruhe, Germany \\
\hline Ponceau S solution & Sigma-Aldrich Co., St. Louis, USA \\
\hline Potassium chloride (KCl) & Carl Roth GmbH \& Co. KG, Karlsruhe, Germany \\
\hline $\begin{array}{l}\text { Potassium ferricyanide (III) } \\
\left(\mathrm{K}_{2} \mathrm{Fe}(\mathrm{CN})_{6}\right)\end{array}$ & Sigma-Aldrich Co., St. Louis, USA \\
\hline $\begin{array}{l}\text { Potassium hexacyanoferrate (II) } \\
\text { trihydrate }\left(\mathrm{K}_{4} \mathrm{Fe}(\mathrm{CN})_{6} \cdot 3 \mathrm{H}_{2} \mathrm{O}\right)\end{array}$ & Sigma-Aldrich Co., St. Louis, USA \\
\hline $\begin{array}{l}\text { Protein G sepharose beads } 4 \text { Fast } \\
\text { Flow }\end{array}$ & Amersham Biosciences, Uppsala, Sweden \\
\hline $\begin{array}{l}\text { Roti-Mount Permanent mounting } \\
\text { medium }\end{array}$ & Carl Roth GmbH \& Co. KG, Karlsruhe, Germany \\
\hline Rotiphorese $^{\circledR}$ Gel 30 & Carl Roth GmbH \& Co. KG, Karlsruhe, Germany \\
\hline Salmon sperm DNA & Stratagene, La Jolla, USA \\
\hline Powdered milk & Carl Roth GmbH \& Co. KG, Karlsruhe, Germany \\
\hline Sodium azide & Sigma-Aldrich Co., St. Louis, USA \\
\hline Sodium chloride $(\mathrm{NaCl})$ & Carl Roth GmbH \& Co. KG, Karlsruhe, Germany \\
\hline Sodium deoxycholate & AppliChem GmbH, Darmstadt, Germany \\
\hline Sodium dodecyl sulfate (SDS) & Carl Roth GmbH \& Co. KG, Karlsruhe, Germany \\
\hline Sodium hydroxide $(\mathrm{NaOH})$ & Carl Roth GmbH \& Co. KG, Karlsruhe, Germany \\
\hline SYBR Green I & Roche Diagnostics $\mathrm{GmbH}$, Mannheim, Germany \\
\hline Tamoxifen (>99\%) & Sigma-Aldrich Co., St. Louis, USA \\
\hline Temgesic $^{\circledR}$ & RB Pharmaceuticals Limited, Berkshire, UK \\
\hline
\end{tabular}




\begin{tabular}{|c|c|}
\hline Tetramethylethylenediamine (TEMED) & Carl Roth GmbH \& Co. KG, Karlsruhe, Germany \\
\hline Tissue-Tek O.C.T ${ }^{\mathrm{TM}}$ Compound & Sakura Finetek USA, Inc., Torrance, USA \\
\hline Tris(hydroxymethyl)aminomethane & Carl Roth GmbH \& Co. KG, Karlsruhe, Germany \\
\hline Triton X-100 & AppliChem GmbH, Darmstadt, Germany \\
\hline Tri-sodium citrate & Carl Roth GmbH \& Co. KG, Karlsruhe, Germany \\
\hline TRIzol ${ }^{\circledR}$ Reagent & Invitrogen $\mathrm{GmbH}$, Karlsruhe , Germany \\
\hline Tween-20 & Carl Roth GmbH \& Co. KG, Karlsruhe, Germany \\
\hline Xylol (Isomere) & Carl Roth GmbH \& Co. KG, Karlsruhe, Germany \\
\hline$X-\beta-G a l$ & Carl Roth GmbH \& Co. KG, Karlsruhe, Germany \\
\hline$\alpha, \alpha-T r e h a l o s e ~ d i h y d r a t e$ & AppliChem GmbH, Darmstadt, Germany \\
\hline$\beta$-Glycerolphosphate (BGP) & Sigma-Aldrich Co., St. Louis, USA \\
\hline
\end{tabular}

Table 4: Enzymes.

\begin{tabular}{|l|l|}
\hline Enzyme & Company \\
\hline M-MuLV Reverse Transcriptase (25 U) & $\begin{array}{l}\text { New England BioLabs GmbH, Frankfurt am Main, } \\
\text { Germany }\end{array}$ \\
\hline Proteinase K & LifeTechnology, Carlsbad, USA \\
\hline RNase inhibitor & $\begin{array}{l}\text { New England BioLabs GmbH, Frankfurt am Main, } \\
\text { Germany }\end{array}$ \\
\hline Taq-Polymerase $(5 \mathrm{U} / \mu \mathrm{l})$ & Primetech, Minsk, Belarus \\
\hline
\end{tabular}

Table 5: Inhibitors.

\begin{tabular}{|l|l|l|}
\hline Inhibitor & Cat. no. & Source \\
\hline $\mathrm{JQ1}\left(\mathrm{C}_{23} \mathrm{H}_{25} \mathrm{CIN}_{4} \mathrm{O}_{2} \mathrm{~S}\right)$ & & $\begin{array}{l}\text { Stefan Knapp, Goethe-University Frankfurt am Main, } \\
\text { Germany }\end{array}$ \\
\hline Ganetespib & $\mathrm{S} 1159$ & Selleckchem \\
\hline
\end{tabular}


MG-132 S2619 Selleckchem

\subsubsection{Cell culture}

Table 6: Cell culture components.

\begin{tabular}{|c|c|}
\hline Component & Company \\
\hline $\begin{array}{l}\text { DMEM, high glucose, HEPES, no } \\
\text { phenol red }\end{array}$ & GIBCO ${ }^{\circledR}$, Invitrogen GmbH, Darmstadt, Germany \\
\hline DMEM/F-12, HEPES, no phenol red & GIBCO $^{\circledR}$, Invitrogen GmbH, Darmstadt, Germany \\
\hline Fetal Bovine Serum (FBS) & Thermo Scientific HyClone, Logan, USA \\
\hline Gibco $^{\circledR} 0.05 \%$ Trypsin-EDTA & GIBCO $^{\circledR}$, Invitrogen $\mathrm{GmbH}$, Darmstadt, Germany \\
\hline McCoy's 5A (Modified) Medium & GIBCO $^{\circledR}$, Invitrogen $\mathrm{GmbH}$, Darmstadt, Germany \\
\hline Opti-MEM & LifeTechnology, Carlsbad, USA \\
\hline Penicillin-Streptomycin & Sigma-Aldrich Co., St. Louis, USA \\
\hline $\begin{array}{l}\text { RPMI } 1640 \text { Medium, GlutaMAX }{ }^{\mathrm{TM}} \\
\text { Supplement }\end{array}$ & $\mathrm{GIBCO}^{\circledR}$, Invitrogen $\mathrm{GmbH}$, Darmstadt, Germany \\
\hline Sodium pyruvate & Sigma-Aldrich Co., St. Louis, USA \\
\hline
\end{tabular}


Table 7: Cell lines.

\begin{tabular}{|c|c|c|c|}
\hline Cell line & Tumor type & Medium & Source \\
\hline COLO201 & $\begin{array}{l}\text { Colorectal } \\
\text { adenocarcinoma }\end{array}$ & $\begin{array}{l}\text { RPMI } \\
\text { GlutaMAXTM }\end{array}$ & $\begin{array}{l}\text { Clinic for General, Visceral and } \\
\text { Pediatric Surgery, UMG }\end{array}$ \\
\hline COLO320DM & $\begin{array}{l}\text { Colorectal } \\
\text { adenocarcinoma }\end{array}$ & $\begin{array}{l}\text { RPMI } \\
\text { GlutaMAX'TM }\end{array}$ & $\begin{array}{l}\text { Clinic for General, Visceral and } \\
\text { Pediatric Surgery, UMG }\end{array}$ \\
\hline DLD1 & $\begin{array}{l}\text { Colorectal } \\
\text { adenocarcinoma }\end{array}$ & $\begin{array}{l}\text { RPMI } \\
\text { GlutaMAXTM }\end{array}$ & Institute of Molecular Oncology, UMG \\
\hline HCT116 & Colorectal carcinoma & McCoy's 5A & Institute of Molecular Oncology, UMG \\
\hline HT-29 & $\begin{array}{l}\text { Colorectal } \\
\text { adenocarcinoma }\end{array}$ & McCoy's 5A & $\begin{array}{l}\text { Clinic for General, Visceral and } \\
\text { Pediatric Surgery, UMG }\end{array}$ \\
\hline LS174T & $\begin{array}{l}\text { Colorectal } \\
\text { adenocarcinoma }\end{array}$ & DMEM/F-12 & Institute of Molecular Oncology, UMG \\
\hline $\mathrm{NCl}-\mathrm{H} 508$ & $\begin{array}{l}\text { Colorectal } \\
\text { adenocarcinoma }\end{array}$ & $\begin{array}{l}\text { RPMI } \\
\text { GlutaMAXTM }\end{array}$ & $\begin{array}{l}\text { Clinic for General, Visceral and } \\
\text { Pediatric Surgery, UMG }\end{array}$ \\
\hline $\mathrm{RKO}$ & Colorectal carcinoma & DMEM/F-12 & Institute of Molecular Oncology, UMG \\
\hline SW48 & $\begin{array}{l}\text { Colorectal } \\
\text { adenocarcinoma }\end{array}$ & DMEM/F-12 & $\begin{array}{l}\text { Clinic for General, Visceral and } \\
\text { Pediatric Surgery, UMG }\end{array}$ \\
\hline SW480 & $\begin{array}{l}\text { Colorectal } \\
\text { adenocarcinoma }\end{array}$ & $\begin{array}{l}\text { RPMI } \\
\text { GlutaMAXTM }\end{array}$ & Institute of Molecular Oncology, UMG \\
\hline SW837 & $\begin{array}{l}\text { Rectal } \\
\text { adenocarcinoma }\end{array}$ & DMEM/F-12 & $\begin{array}{l}\text { Clinic for General, Visceral and } \\
\text { Pediatric Surgery, UMG }\end{array}$ \\
\hline T84 & Colorectal carcinoma & DMEM/F-12 & $\begin{array}{l}\text { Clinic for General, Visceral and } \\
\text { Pediatric Surgery, UMG }\end{array}$ \\
\hline
\end{tabular}

\subsubsection{Kits}

Table 8: Kits.

\begin{tabular}{|l|l|}
\hline Kit & Company \\
\hline Agilent High Sensitivity DNA Kit & Agilent Technology, Santa Clara, USA \\
\hline
\end{tabular}




\begin{tabular}{|l|l|}
\hline Bioanalyzer DNA High sensitivity kit & Agilent Technologies, Santa Clara, USA \\
\hline $\begin{array}{l}\text { Immobilon Western Blot HRP Substrate } \\
\text { Luminol Reagent }\end{array}$ & Merck Millipore, Darmstadt, Germany \\
\hline $\begin{array}{l}\text { NEXTflex } \\
\text { RNA Rapeq Library Prep Kit }\end{array}$ & Bioo Scientific Corporation, Austin, USA \\
\hline Pierce $^{\text {TM }}$ BCA Protein Assay Kit & ThermoFisher Scientific, Rockford, USA \\
\hline Qubit dsDNA HS assay & Invitrogen GmbH, Karlsruhe, Germany \\
\hline SuperSignal ${ }^{\circledR}$ West Femto Maximum & Thermo Fisher Scientific, Waltham, USA \\
\hline
\end{tabular}

\subsubsection{Oligonucleotides}

Table 9: Primers used to genotype experimental mice.

\begin{tabular}{|c|c|c|c|c|}
\hline $\begin{array}{l}\text { Mouse } \\
\text { line }\end{array}$ & Gene & $\begin{array}{l}\text { Primer } \\
\text { name }\end{array}$ & Sequence (5'-3') & Source \\
\hline \multirow{3}{*}{ Usp22lacz } & \multirow{3}{*}{ Usp22 } & LoxP F & CCCAGCTTTCTTGTACAAAGTGGTT & \multirow{3}{*}{$\begin{array}{l}\text { (Kosinsky et } \\
\text { al., 2015) }\end{array}$} \\
\hline & & Wt F & GTGCCCTGGTTGCCCAGTGAG & \\
\hline & & LoxP/wt R & CGGTTCAGGTGGATGCCGCA & \\
\hline \multirow{3}{*}{$\mathrm{APC}^{1638 \mathrm{~N}}$} & \multirow{3}{*}{$A p c$} & Apc-A3 & CTAGCCCAGACTGCTTCAAAAT & \multirow{3}{*}{$\begin{array}{l}\text { (Cremers et al., } \\
2016)\end{array}$} \\
\hline & & Apc-C2 & GGAAAAGTTTATAGGTGTCCCTTCT & \\
\hline & & PN3 & GCCAGCTCATTCCTCCACTC & \\
\hline \multirow{2}{*}{ Usp22 loxP } & \multirow{2}{*}{ Usp22 } & $\begin{array}{l}\text { Usp22 } \\
6720 \mathrm{~F}\end{array}$ & TGTGCCCTGGTTGCCCAGTGA & \multirow{2}{*}{ This study } \\
\hline & & $\begin{array}{l}\text { Usp22 } \\
\text { 14079R }\end{array}$ & GCACCACCACAGCCGTCCTT & \\
\hline \multirow{2}{*}{$\begin{array}{l}\text { Villin- } \\
\text { Cre }\end{array}$} & \multirow{2}{*}{$\begin{array}{l}\text { Villin- } \\
\text { Cre }\end{array}$} & $\begin{array}{l}\text { Villin- } \\
\text { 2kbseqS }\end{array}$ & CAAGCCTGGCTCGACGGCC & \multirow{2}{*}{$\begin{array}{l}\text { (Donovan et } \\
\text { al., 2005) }\end{array}$} \\
\hline & & Villin-Cre198 & CGCGAACATCTTCAGGTTCT & \\
\hline
\end{tabular}


Table 10: qRT-PCR primers for gene expression studies.

\begin{tabular}{|c|c|c|c|c|}
\hline Gene & $\begin{array}{l}\text { Primer } \\
\text { name }\end{array}$ & Sequence (5'-3') & Organism & Source \\
\hline \multirow{2}{*}{$18 S$ rRNA } & $\begin{array}{l}\text { h1SrRNA } \\
\text { 903F }\end{array}$ & AACTGAGGCCATGATTAAGA & Human & \multirow{2}{*}{$\begin{array}{l}\text { (Upasana Bedi, } \\
\text { 2013) }\end{array}$} \\
\hline & $\begin{array}{l}\text { h18SrRNA } \\
\text { 1075R }\end{array}$ & GGAACTACGACGGTATCTGA & Human & \\
\hline \multirow{2}{*}{$36 B 4$} & m36B4F & GATTGGCTACCCAACTGTTG & Mouse & \multirow{2}{*}{$\begin{array}{l}\text { (Mesange et al., } \\
\text { 2014) }\end{array}$} \\
\hline & m36B4R & CAGGGGCAGCAGCCACAAA & Mouse & \\
\hline \multirow{2}{*}{ HSP90AB1 } & $\begin{array}{l}\text { hHSP90AB1 } \\
469 F\end{array}$ & TTGACATCATCCССАAСССТC & Human & \multirow{2}{*}{ This study } \\
\hline & $\begin{array}{l}\text { hHSP90AB1 } \\
641 \mathrm{R}\end{array}$ & ACCAAACTGCCCAATCATGGA & Human & \\
\hline \multirow{2}{*}{ USP22 } & $\begin{array}{l}\text { hUsp22 } \\
\text { 2792F }\end{array}$ & AGCCAAGGGTGTTGGTCGCG & Human & \multirow{2}{*}{$\begin{array}{l}\text { (Theresa Gorsler, } \\
\text { 2013) }\end{array}$} \\
\hline & $\begin{array}{l}\text { hUsp22 } \\
\text { 2897R }\end{array}$ & ACTGCCACCACGCCCGAAAG & Human & \\
\hline \multirow{2}{*}{ Usp22 } & $\begin{array}{l}\text { mUsp22 } \\
\text { 189F }\end{array}$ & GGAGCCTGAGGTCGAGGCCA & Mouse & \multirow{2}{*}{ This study } \\
\hline & $\begin{array}{l}\text { mUsp22 } \\
\text { 359R }\end{array}$ & ACACAGGACTTTGCCTTGCGC & Mouse & \\
\hline
\end{tabular}

Table 11: siRNAs used for transient gene silencing in vitro.

\begin{tabular}{|l|l|l|l|}
\hline Gene & Sequence $\left(\mathbf{5}^{\prime} \mathbf{3}^{\prime} \mathbf{3}^{\prime}\right)$ & Cat. No. & Company \\
\hline NT5 & - & D-001210-05 & Dharmacon \\
\hline \multirow{3}{*}{ USP22 } & GGAAGAAAGAUCACCUCGAA & & \\
\cline { 2 - 2 } & CAAAGCAGCUCACUAUGAA & & \\
\cline { 2 - 3 } & GGAAGAUCACCAACGUAUGU & MU-006072-01-0010 & Dharmacon \\
\cline { 2 - 3 } & CCUUUAGUCUCAAGAGCGA & & \\
\hline
\end{tabular}




\subsubsection{Antibodies}

Table 12: Primary antibodies used for western blot, Co-IP and IHC.

\begin{tabular}{|l|l|l|l|l|l|l|}
\hline Antibody & Host & Cat. no. & Source & $\begin{array}{l}\text { Western } \\
\text { blot }\end{array}$ & Co-IP & IHC \\
\hline CTIP2 & Rat & ab18465 & Abcam & & & $1: 100$ \\
\hline GAPDH & Mouse & ab8245 & Abcam & $1: 1,000$ & & \\
\hline H2B & Mouse & ab52484 & Abcam & $1: 20,000$ & & \\
\hline H2B & Rabbit & ab1790 & Abcam & & & $1: 1,000$ \\
\hline H2Bub1 & Mouse & \multicolumn{1}{|l}{ (Prenzel et al., 2011) } & $01: 10$ & & $01: 20$ \\
\hline HSP90AB1 (clone 4C10) & Mouse & TA500494 & OriGene & $1: 5,000$ & $2 \mu \mathrm{g}$ & \\
\hline IgG & Mouse & $\begin{array}{l}015-000- \\
003\end{array}$ & Dianova & & $2 \mu \mathrm{g}$ & \\
\hline SATB2 & Rabbit & $2819-1$ & Epitomics & & & $1: 100$ \\
\hline TBR2 & Rabbit & ab2283 & Millipore & & & $1: 100$ \\
\hline USP22 & Mouse & sc 390585 & Santa Cruz & $1: 1,000$ & $4 \mu g$ & \\
\hline
\end{tabular}

Table 13: Secondary antibodies.

\begin{tabular}{|l|l|l|l|l|l|}
\hline Antibody & Host & Cat. no. & Source & $\begin{array}{l}\text { Western } \\
\text { blot }\end{array}$ & IHC \\
\hline Anti-mouse IgG, biotinylated & Sheep & RPN1001 & $\begin{array}{l}\text { GE } \\
\text { Healthcare }\end{array}$ & $1: 1,000$ \\
\hline Anti-rabbit IgG, biotinylated & Donkey & RPN1004 & $\begin{array}{l}\text { GE } \\
\text { Healthcare }\end{array}$ & $1: 1,000$ \\
\hline goat-anti-mouse IgG-HRP & goat & sc-2005 & Santa Cruz & $1: 10,000$ & $1: 10,000$ \\
\hline goat-anti-rabbit IgG-HRP & goat & sc-2004 & Santa Cruz & \\
\hline
\end{tabular}




\subsubsection{Buffers}

\section{2x qPCR mix}

$75 \mathrm{mM}$ Tris/ $\mathrm{HCl}, 20 \mathrm{mM}\left(\mathrm{NH}_{4}\right)_{2} \mathrm{SO}_{4}, 0.01 \%$ Tween 20, $3 \mathrm{mM} \mathrm{MgCl}_{2}, 0.2 \mathrm{mM}$ dNTPs, $0.25 \%$ Triton X-100, $20 \mathrm{U} / \mathrm{ml}$ Taq polymerase, 1:80,000 SYBR Green I, 300 mM Trehalose, pH 8.8

\section{Citric acid buffer}

$12 \mathrm{mM}$ citric acid, $100 \mathrm{mM}$ tri-sodium citrate, $\mathrm{pH} 6.0$

\section{Crystal violet solution}

$0.1 \%(\mathrm{w} / \mathrm{v})$ crystal violet in $20 \% \mathrm{EtOH}$

\section{DNA extraction buffer}

$10 \mathrm{mM}$ Tris/HCl, $400 \mathrm{mM} \mathrm{NaCl}, 2 \mathrm{mM}$ EDTA, 2\% SDS, $10 \mu \mathrm{g} / \mathrm{ml}$ proteinase $\mathrm{K}$

DNA loading dye (6x)

$40 \%(w / v)$ sucrose, $10 \%(v / v)$ glycerol, $0.25 \%(w / v)$ bromophenol blue

\section{E1A buffer}

50 mM HEPES, 150 mM NaCl, 0.1\% NP-40, pH 7.3

\section{Embryo fixation solution}

$6.75 \mathrm{ml} \mathrm{37 \%}$ formaldehyde, $2 \mathrm{ml} 25 \%$ glutaraldehyde, $5 \mathrm{ml} \mathrm{NP}-40,25 \mathrm{ml}$ 10x PBS, filled up to $250 \mathrm{ml}$ with $\mathrm{H}_{2} \mathrm{O}, 110 \mathrm{mg} \mathrm{DOC}$

\section{Firefly buffer}

$25 \mathrm{mM}$ glycylglycine, $15 \mathrm{mM} \mathrm{K}_{2} \mathrm{HPO}_{4}, 4 \mathrm{mM}$ EGTA, $15 \mathrm{mM} \mathrm{MgSO}_{4}, 4 \mathrm{mM} \mathrm{ATP}, 1.25 \mathrm{mM}$ DTT, $0.1 \mathrm{mM}$ CoA, $80 \mu \mathrm{M}$ luciferin, $\mathrm{pH} 8.0$

\section{Laemmli buffer (6x)}

9.3\% (w/v) DTT, $0.02 \%(w / v)$ bromophenol blue, 30\% (v/v) glycerol, 10\% (w/v) SDS, $0.35 \mathrm{M}$ Tris, $\mathrm{pH} 6.8$

\section{PBS}

$137 \mathrm{mM} \mathrm{NaCl}, 4.29 \mathrm{mM} \mathrm{Na}_{2} \mathrm{HPO}_{4} .2 \mathrm{H}_{2} \mathrm{O}, 2.68 \mathrm{mM} \mathrm{KCl}, 1.47 \mathrm{mM} \mathrm{KH}_{2} \mathrm{PO}_{4}, \mathrm{pH} 7.4$

\section{Renilla buffer}

1.1 M NaCl, $2.2 \mathrm{mM} \mathrm{Na}_{2}$ EDTA, 0.22 $\mathrm{M} \mathrm{K}_{2} \mathrm{HPO}_{4}, 1.5 \mathrm{mM} \mathrm{NaN}_{3}, 0.5 \mathrm{mg} / \mathrm{ml}$ BSA, $1.5 \mu \mathrm{M}$ Coelenterazine, $\mathrm{pH} 5.1$ 


\section{RIPA buffer}

$1 \%$ NP-40, $0.1 \%$ SDS, $0.5 \%$ sodium deoxycholate in PBS

\section{RNA loading dye}

$0.1 \%(\mathrm{w} / \mathrm{v})$ bromophenol blue, $49.9 \%$ DEPC water, $50 \%$ glycerol $(\mathrm{w} / \mathrm{v})$

\section{SDS running buffer}

$25 \mathrm{mM}$ Tris, $86 \mathrm{mM}$ glycine, $3.5 \mathrm{mM}$ SDS

\section{SDS separating gel (10\%)}

$33 \%(\mathrm{v} / \mathrm{v})$ acrylamide, $0.1 \%(\mathrm{w} / \mathrm{v})$ APS, $0.1 \%(\mathrm{w} / \mathrm{v})$ SDS, 375mM Tris/HCl, $0.04 \%(\mathrm{v} / \mathrm{v})$ TEMED, pH 8.8

\section{SDS stacking gel}

$5 \%(\mathrm{v} / \mathrm{v})$ acrylamide, $0.1 \%(\mathrm{w} / \mathrm{v})$ APS, $0.1 \%(\mathrm{w} / \mathrm{v})$ SDS, $125.5 \mathrm{mM}$ Tris/ $\mathrm{HCl}, 0.1 \%(\mathrm{v} / \mathrm{v})$ TEMED, $\mathrm{pH} 6.8$

\section{TBE buffer}

$45 \mathrm{mM}$ Tris, $1 \mathrm{mM} \mathrm{Na} \mathrm{EDTA}, 45 \mathrm{mM}$ boric acid

Western blot transfer buffer

$10 \%(\mathrm{v} / \mathrm{v}) 10 \mathrm{x}$ western salts, $20 \%(\mathrm{v} / \mathrm{v})$ methanol

Western salts (10x)

1.92 M glycine, 0.02\% (w/v) SDS, 250 mM Tris/HCl, pH 8.3

\section{X-gal staining solution}

$2.5 \% 250 \mathrm{mM} \mathrm{K}_{3} \mathrm{Fe}(\mathrm{CN})_{6}, 2.5 \% 250 \mathrm{mM} \mathrm{K}_{4} \mathrm{Fe}(\mathrm{CN})_{6}, 2 \% 100 \mathrm{mM} \mathrm{MgCl}_{2}$ in PBS 


\subsubsection{Software and tools}

Table 14: Software and tools.

\begin{tabular}{|c|c|}
\hline Software/Tool & Source \\
\hline AxioCam MRc and AxioVision 4.8 Software & Carl Zeiss \\
\hline Bio-Rad CFX Manager 3.1 & Bio-Rad Laboratories, Hercules, USA \\
\hline cBioPortal & $\begin{array}{l}\text { http://www.cbioportal.org/ } \\
\text { (Cerami et al., 2012; Gao et al., 2013), }\end{array}$ \\
\hline CFX Manager Software 3.1 for qPCR cycler & Bio-Rad \\
\hline FIJI & (Schindelin et al., 2012) \\
\hline Galaxy & http://usegalaxy.org/ \\
\hline GOTermFinder & $\begin{array}{l}\text { Princeton University, Lewis-Sigler Institute, } \\
\text { http://go.princeton.edu/cgi-bin/GOTermFinder }\end{array}$ \\
\hline GraphPad Prism & $\begin{array}{l}\text { GraphPad Prism version } 5 \text { for Windows, } \\
\text { GraphPad Software, Inc., San Diego, USA }\end{array}$ \\
\hline Image Lab Version 5.2 build 14 & Bio-Rad Laboratories, Hercules, USA \\
\hline IST Online ${ }^{\circledR}$ & MediSapiens Ltd., http://ist.medisapiens.com/ \\
\hline Microsoft Excel, Word, PowerPoint & Microsoft, Redmond, USA \\
\hline Morpheus & https://software.broadinstitute.org/morpheus/ \\
\hline Oncomine $^{\mathrm{TM}}$ & $\begin{array}{l}\text { https://www.oncomine.org/resource/login.html } \\
\text { (Rhodes et al., 2004) }\end{array}$ \\
\hline Primer designing tool NCBI/Primer-BLAST & (Ye et al., 2012) \\
\hline REVIGO & http://revigo.irb.hr/ (Supek et al., 2011) \\
\hline Statistical software $R$ & $\begin{array}{l}\text { R: A language and environment for statistical } \\
\text { computing. R Foundation for Statistical } \\
\text { Computing, 2013, https://www.r-project.org/ }\end{array}$ \\
\hline TestXpert software & Zwick GmbH \& Co. KG, Ulm, Germany \\
\hline uEYE Cockpit & $\begin{array}{l}\text { IDS Imaging Development Systems GmbH, } \\
\text { Obersulm, Germany }\end{array}$ \\
\hline
\end{tabular}




\subsection{Methods}

\subsubsection{Animal studies}

\subsubsection{Generation of mice and genotyping}

C57BL/6 embryonic stem cells (ESCs) expressing the LacZ gene under the control of the endogenous Usp22 promoter were obtained from the University-Davis Knockout Mouse Project Repository (clone Usp22_D11). In Usp22 lacz mice stop codons and poly-A sites result in a reduced Usp22 expression as previously described (Kosinsky et al., 2015). By FLPmediated excision the lacZ and neomycin resistance locus were removed from the construct in order to generate Usp22 $2^{\text {loxP }}$ animals in which a conditional knockout of Usp22 was possible. Usp22 $2^{10 \times P}$ mice were crossed with Villin-Cre ${ }^{E R T 2}$ and APC ${ }^{1638 N}$ animals to achieve an intestinal knockout and to promote tumorigenesis, respectively. Mice in this study were on the C57BL/6J background.

\subsubsection{Tamoxifen injection}

$5 \%$ Tamoxifen $(\mathrm{w} / \mathrm{v})$ was dissolved in $100 \% \mathrm{EtOH}$. Right before intraperitoneal injection into mice, this solution was mixed 1:10 with sunflower oil. Mice were injected at an age of 4 weeks for five consecutive days with a total dose of $1 \mathrm{mg}$ Tamoxifen per day. Mice injected at an age of 14 weeks received $1.5 \mathrm{mg}$ per day.

\subsubsection{DSS administration}

10 week-old mice were treated with the chemical agent dextran sulfate sodium (DSS) in order to trigger colitis and the subsequent formation of colorectal tumors. $2 \%$ DSS (w/v) was dissolved in drinking water and the solution was refreshed every two to three days. Since the treatment can be accompanied by diarrhea, intestinal bleedings and weight loss, the administration of DSS was segmented into two cycles. After four days of being treated with DSS, mice were put on a special diet using soft food and glucose for one week. During this 
week the animals recovered and were treated with DSS for three days afterwards. At the end of the treatment the mice received soft food again for three weeks. Animals were injected with Temgesic $^{\circledR}$ (buprenorphine) up to three times per day for a total duration of approximately 7 days to prevent/treat abdominal pain starting on the last day of DSS treatment. The body weight of the mice and intestinal bleeding intensities were checked daily using the stool guaiac test.

\subsubsection{Stool guaiac test}

To assess the presence and intensity of intestinal bleedings caused by DSS treatment, the stool guaiac test was carried out. Guaiac resin was dissolved in $70 \% \mathrm{EtOH}$ until the solution was saturated. A few drops were applied to a Whatman filter paper and left for drying at room temperature for at least 30 minutes. Feces were applied and distributed on the dried Guaiac solution. Upon $\mathrm{H}_{2} \mathrm{O}_{2}$ addition, blue staining was observed in the presence of blood which was scored according to its intensity from 3 to 0 with decreasing intensity or absence, respectively.

\subsubsection{Determination of disease activity index (DAI)}

To determine the severity of colitis the disease activity index (DAI) was calculated by scoring three aspects, i.e. weight loss, stool consistency and intestinal bleeding intensity. Weight loss: $0-1 \%(0), 1-5 \%(1), 5-10 \%(2), 10-15 \%(3),>15 \%$ (4). Stool consistency: normal (0), soft (1), very soft (2), diarrhea (3). To evaluate intestinal bleeding intensity the stool guaiac test was performed. Scoring of the stool guaiac test was: no blue staining (0), weak, sporadic staining (1), medium (2) and strong blue staining (3), bloody anus (4). After adding the scores per aspect maximum score of 11 can be reached per day. Animals which had to be sacrificed due to severe symptoms received a score of 12 . 


\subsubsection{Tissue isolation}

Mice were euthanized by $\mathrm{CO}_{2}$ and cervical dislocation. They were checked for the presence of metastases and further abnormalities such as intussusceptions. Intestines were flushed with PBS and cut open longitudinally. Tumors were counted and their location and size was measured. Some tumors were isolated and frozen. Subsequently intestines were rolled upwards resulting in their wrapping around themselves to form "Swiss rolls". The distal third of the SI and the complete colon were fixed in 4\% formaldehyde in PBS overnight for subsequent paraffin embedding. The proximal third of the SI was frozen at $-20^{\circ} \mathrm{C}$ in Tissue-Tek O.C. $\mathrm{T}^{\mathrm{TM}}$ cryo embedding medium and the remaining third was snap-frozen in liquid nitrogen and stored at $-80^{\circ} \mathrm{C}$ for subsequent protein or RNA isolation.

\subsubsection{Serum isolation}

After sacrificing animals, the heart was punctured and blood was isolated. It was stored at room temperature for $1 \mathrm{~h}$ and spun down at $1,000 \mathrm{rpm}$ for $10 \mathrm{~min}$. The serum was transferred to a new tube and if blood color varied among animals, the hematocrit/serum fractions were weighed and the ratio was calculated. Subsequently, the serum was stored at $-20^{\circ} \mathrm{C}$.

\subsubsection{Isolation of intestinal epithelial cells}

Intestines were flushed with PBS and cut longitudinally. 1-2 cm long fragments were prepared and vortexed in ice-cold PBS for $1 \mathrm{~min}$. If the intestines were still dirty or covered by mucus, PBS was decanted, $4.5 \mathrm{mM}$ DTT in PBS was added and after shaking at room temperature for 10 min, tissues were transferred to fresh PBS. To separate the epithelium from the muscle layers, intestinal fragments were shaken in $20 \mathrm{ml} 5 \mathrm{mM}$ EDTA in PBS for $15 \mathrm{~min}$. After vortexing for $2 \mathrm{~min}$, the supernatant containing the epithelial cells was transferred into a clean $50 \mathrm{ml}$ tube containing $20 \mathrm{ml} 5 \mathrm{mM}$ EDTA in PBS. These steps were repeated until the solution was clear and no villi were visible under the microscope. Supernatants were combined and spun down 
at $1,000 \mathrm{rpm}$ for $10 \mathrm{~min}$. Pellets were washed with $\mathrm{PBS}$ and stored at $-80^{\circ} \mathrm{C}$ for subsequent RNA and protein isolation.

\subsubsection{X-gal staining of embryos}

After heterozygous mating, Usp22 $2^{\text {lacz }}$ embryos at E10.5 and E15.5 were collected and fixed in fixation solution on ice for $60 \mathrm{~min}$. X-gal staining solution was pre-warmed to $37^{\circ} \mathrm{C}$ and $\mathrm{X}$-gal solution (40 mg X- $\beta$-Gal/ml dimethylformamide) was added. After washing in PBS, embryos were incubated in staining solution protected from light for $24 \mathrm{~h}$. Subsequently, they were embedded in Tissue-Tek O.C.T $\mathrm{TM}^{\mathrm{TM}}$ and cut on the sagittal plane. The frozen embryos were thawed in $0.1 \%$ PFA in PBS for approximately $10 \mathrm{~min}$. Afterwards they were washed, dehydrated and prepared for paraffin embedding. $20 \mu \mathrm{m}$ sections were counterstained using nuclear fast red-aluminum sulfate solution.

\subsubsection{Preparation of mouse embryonic fibroblasts (MEFs)}

After heterozygous mating the uterus of pregnant Usp22 $2^{\text {lacz }}$ mice was removed at E13.5 and transferred into ice-cold PBS. Under sterile conditions the uterus and amnion were removed and embryos were washed in PBS. Liver, tail and limbs were removed and the head was lysed for genotyping. Tissues were homogenized in $5 \mathrm{ml} \mathrm{0.05 \%} \mathrm{Trypsin-EDTA} \mathrm{with} \mathrm{a} \mathrm{sterile} \mathrm{scalpel.}$ After incubating at $37^{\circ} \mathrm{C}$ for $5-10 \mathrm{~min}$, the tissue was resuspended and incubated for further 5 min. $5 \mathrm{ml}$ DMEM was added and following inverting, tubes were centrifuged at 1,000 rpm for 7 min. The pellet was resuspended in $5 \mathrm{ml}$ warm DMEM supplemented with $10 \% \mathrm{FBS}, 1 \%$ sodium pyruvate, 100 units $/ \mathrm{ml}$ penicillin, and $100 \mu \mathrm{g} / \mathrm{ml}$ streptomycin and transferred into a 15 $\mathrm{cm}$ dish containing $10 \mathrm{ml}$ DMEM. Cells were incubated at $37^{\circ} \mathrm{C}, 5 \% \mathrm{CO}_{2}$ until plates were confluent (24-72h). 


\subsubsection{Preparation of paraffin-embedded tissue}

After formaldehyde fixation, organs were washed using tap water and PBS and dehydrated in 70\% EtOH overnight. Dehydration proceeded using 80\%, 90\% and 100\% EtOH with $1 \mathrm{~h}$ shaking for each. EtOH was exchanged with isopropanol and tissues were shaken overnight. Isopropanol was slowly exchanged with xylol by decreasing the isopropanol and increasing the xylol ratio. After shaking the organs in pure xylol, tissues were transferred to paraffin at $62^{\circ} \mathrm{C}$ for three days. Finally tissues were embedded in paraffin blocks which were used to prepare 5 $\mu \mathrm{m}$ sections.

\subsubsection{H\&E and Nissl staining}

Organ sections were de-paraffinized in xylol for $20 \mathrm{~min}$ and rehydrated by storing them in decreasing $\mathrm{EtOH}$ solutions (100\%, 90\%, 70\%) each for 5 min. Slides were washed with water and nuclei were stained in Mayer's hematoxylin solution for $1 \mathrm{~min}$. Excess dye was removed by rinsing slides under running tap water for 5 min. Counterstaining was performed using Eosin for 5-10 min. Slides were washed and dehydrated in increasing concentrations of $\mathrm{EtOH}$ solutions. After incubation in xylol for $10 \mathrm{~min}$, mounting medium and cover slips were added to slides. For morphological analyses 100-200 crypts/villi were assessed. For Nissl staining of brain material, sections were de-paraffinized, rehydrated and stained in $0.5 \%$ cresyl violet for 10 min. For rehydration and mounting, the aforementioned steps were followed. Nissl staining was performed in cooperation with Dr. Nicole Hellbach and Prof. Dr. Tanja Vogel (Department of Molecular Embryology, Institute of Anatomy and Cell Biology, Faculty of Medicine, University of Freiburg, Germany). 


\subsubsection{Immunohistochemistry (IHC)}

Similar to H\&E staining, sections were de-paraffinized and rehydrated. Antigen retrieval was performed by boiling slides in $10 \mathrm{mM}$ citric acid buffer or $1 \mathrm{mM}$ EDTA for $15 \mathrm{~min}$. Sections were quenched for endogenous peroxidases with $5 \% \mathrm{H}_{2} \mathrm{O}_{2}$ in PBS and blocked with $10 \%$ fetal bovine serum (FBS) in PBS. Primary antibodies were diluted in 10\% FBS/PBS and incubated overnight at $4^{\circ} \mathrm{C}$. Biotinylated secondary antibodies (1:200) and ExtrAvidin-Peroxidase $(1: 1,000)$ were added each for $1 \mathrm{~h}$. Staining was developed using DAB and counterstaining was carried out using hematoxylin.

\subsubsection{Histo-score (H-score)}

The Histo-score (H-score) is based on the intactness of the intestinal epithelium and the rate of lymphocyte infiltration to determine inflammation intensity. The $\mathrm{H}$-score is assigned in a range from 0 to 3 with an increasing disruption of crypt structure. In contrast to the normal and healthy intestinal epithelium (score 0), mild inflammation and therefore lymphocyte infiltration can cause crypts to be pushed apart from each other (1). While the presence of MALTs below the crypts are part of the normal immune defense, during colitis lymphocyte accumulations can destroy crypt structure (2). The highest score is assigned when the epithelial lining on top of the lymphocyte accumulation has been destroyed (3). The percentage of damaged tissue was multiplied by the respective score (0-3) and the sum was divided by the maximum $\mathrm{H}$-score, which was 30 . This method is based on a colitis-scoring technique established by Dr. med. Hanibal Bohnenberger (Institute for Pathology, UMG) and Garrit Meers (Institute for Cellular and Molecular Immunology, University of Göttingen Medical School) and has been modified accordingly for (colitis-induced) colorectal cancer sections. 


\subsubsection{Mechanical bone testing}

To determine bone biomechanical properties, femora were located on a Zwick device while a stamper moved towards the bone with $50 \mathrm{~mm} / \mathrm{min}$. Initially, a primary force of $1 \mathrm{~N}$ was applied to fix the bone on the plate. Afterwards, measurements were performed with an accuracy of 0.2-0.4\% using 2-500 N. Data were recorded while the applied strength was linear and was aborted once the curve declined by $10 \mathrm{~N}$. By means of the testXpert software the applied strength when the deformation was induced (yield load), during fracture (Fmax), before breaking the bone (failure load) and the bone stiffness were determined (Komrakova et al., 2010). Analyses were performed in cooperation with Dr. med. Dominik Saul (Department of Trauma, Orthopedics and Reconstructive Surgery, UMG).

\subsubsection{Cell culture}

\subsubsection{Cell culture and inhibitor treatment}

Human colorectal cancer cell lines were grown in phenol-red free Dulbecco's Modified Eagle's Medium (DMEM/F-12), RPMI or McCoy's 5A medium supplemented with $10 \%$ fetal bovine serum, $100 \mathrm{units} / \mathrm{ml}$ penicillin and $100 \mu \mathrm{g} / \mathrm{ml}$ streptomycin at $37^{\circ} \mathrm{C}$ and $5 \% \mathrm{CO}_{2}$. Inhibitors were dissolved in DMSO. Increasing concentrations of Ganetespib and JQ1 were added to the cells for $48 \mathrm{~h}$. Cells were incubated with $20 \mu \mathrm{M}$ of the proteasome inhibitor MG-132 for $4 \mathrm{~h}$. As a negative control DMSO was added to the cells.

\subsubsection{2 siRNA transfection}

siRNA transfections were performed using Lipofectamine ${ }^{\circledR}$ RNAiMAX according to the manufacturer's instructions. Briefly, 30 pmol siRNA was mixed with $500 \mu \mathrm{l}$ of Opti-MEM and 5 $\mu l$ of Lipofectamine ${ }^{\circledR}$ RNAiMAX. After incubating the transfection mix at room temperature for $20 \mathrm{~min}$, it was added to a well of a 6 -well plate containing 400,000 cells in $1.5 \mathrm{ml}$ medium without antibiotics. For the determination of cells' characteristics, cells were trypsinized $24 \mathrm{~h}$ 
post transfection and subjected to a variety of assays. When isolating protein or RNA of siRNAtreated cells, $24 \mathrm{~h}$ after transfection medium containing antibiotics was added and $48 \mathrm{~h}$ later cells were harvested.

\subsubsection{Proliferation assessment}

To assess proliferation rates, 2,000-5,000 cells were seeded per well of a 96-well plate after siRNA transfection. Proliferation during inhibitor treatment was assessed in 24-well plates. Confluence was measured every $24 \mathrm{~h}$ using a Celigo ${ }^{\circledR}$ Adherent Cell Cytometer for one week. Alternatively, 30,000-75,000 cells were seeded per well of a 12-well plate. After $48 \mathrm{~h}$ cells were washed with PBS and fixed with 4\% PFA in PBS for 20 min. Upon a further washing step, cells were visualized by crystal violet staining for 20 minutes. Excess dye was removed by rinsing wells several times with water and plates were scanned.

\subsubsection{Migration assay}

Migration potential of cells was assessed using a trans-well migration assay as previously described (Prenzel et al., 2011). Briefly, 48 hours after transfection with siRNAs, 50,000 HCT116 or 75,000 SW48 cells, respectively, were seeded into 8.0 - $\mu$ m PET track-etched membrane cell culture inserts. Cells were grown for another 48 hours $37^{\circ} \mathrm{C}$ and $5 \% \mathrm{CO}_{2}$ before fixation with methanol for 10 minutes. Migrated cells were visualized by crystal violet staining for 20 minutes. Finally, inserts were rinsed with water, allowed to dry and scanned.

\subsubsection{Colony formation assay}

After trypsinization, 500 cells were transferred per well of a 6-well plate. Approximately 4-7 days later when colonies were detected macroscopically, colonies were stained using crystal violet as explained before. 


\subsubsection{Soft agar colony formation assay}

This experiment was performed to assess the ability of cells to grow in an anchorageindependent manner. Initially, a sterile stock agarose solution (3.2\% in water) was prepared and autoclaved. It was then mixed with the respective growth medium and $1 \mathrm{ml}$ was transferred per well of a 6 -well plate to prepare a $0.8 \%$ base agarose layer. Subsequently, $150 \mu$ of the $3.2 \%$ pre-warmed $\left(37-40^{\circ} \mathrm{C}\right)$ agarose solution was added to 11,500 cells in $1 \mathrm{ml}$ of growth medium. $750 \mu \mathrm{l}$ of this mixture was transferred immediately on top of the base agarose layer. Upon solidification, 1-2 $\mathrm{ml}$ of normal growth medium was added and cells were incubated at $37^{\circ} \mathrm{C}$ for $10-20$ days until colonies were visible macroscopically. Cells were fixed with $4 \%$ PFA in PBS for 20 min and subsequently stained with $0.005 \%$ crystal violet for $1 \mathrm{~h}$. After carefully rinsing the wells with water, plates were scanned.

\subsubsection{CRISPR/Cas9-mediated knockout of USP22}

In order to achieve a permanent knockout of USP22 in HCT116 cells, 400,000 cells were seeded per well of a 6-well plate. Single guide RNAs (sgRNAs) targeting USP22 were designed by Dr. rer. nat. Florian Wegwitz (Department of General, Visceral and Pediatric Surgery, UMG) with the help of the E-CRISP algorithm from the German Cancer Research Center (http://www.e-crisp.org). The selected guide RNA sequences were then cloned into the pSpCas9(BB)-2A-GFP (PX458, Addgene) vector. $2.5 \mu \mathrm{g}$ plasmid containing Cas9 and GFP sequences as well as a sgRNA were complexed with Lipofectamine ${ }^{\circledR} 2000$ for 25 min at room temperature and added to the wells containing medium without antibiotics. After $24 \mathrm{~h}$ cells were washed with PBS and medium supplemented with antibiotics was added. $48 \mathrm{~h}$ after transfection the cells were sorted based on their fluorescence intensity via fluorescence activated cell sorting (FACS). FACS was performed by Sabrina Becker (Cell-sorting technology platform, Department of Haematology and Medical Oncology, UMG). Single highly fluorescent cells were 
sorted into the wells of a 96 well plate. Cell clones were cultivated and propagated. The loss of USP22 was confirmed at the protein level by western blot.

\subsubsection{Molecular biology techniques}

\subsubsection{DNA extraction from tail biopsies or cells}

Tail biopsies or cell pellets were lysed at $56^{\circ} \mathrm{C}$ in DNA extraction buffer overnight. To separate nucleic acids from other cellular contaminants, $100 \mu \mathrm{l} 5 \mathrm{M} \mathrm{NaCl}$ was added. Tubes were inverted and centrifuged at room temperature and maximum speed for $10 \mathrm{~min}$. An equal volume of isopropanol was added to the supernatant in a new tube and after mixing samples they were spun down at $4^{\circ} \mathrm{C}$ to precipitate the DNA. The pellet was washed with $100 \mu$ icecold $70 \% \mathrm{EtOH}$ and spun down. Following EtOH removal, DNA pellets were air-dried and dissolved in $50 \mu \mathrm{H} \mathrm{H}_{2} \mathrm{O}$.

\subsubsection{Genotyping of experimental mice}

Genotyping of Usp22 $2^{\text {lacz }}$ mice was performed by pre-heating PCR samples to $95^{\circ} \mathrm{C}$ for $3 \mathrm{~min}$. The respective DNA fragments were amplified in 35 polymerization cycles with $95^{\circ} \mathrm{C}$ for $30 \mathrm{~s}$, $60^{\circ} \mathrm{C}$ for $30 \mathrm{~s}, 72^{\circ} \mathrm{C}$ for $1 \mathrm{~min}$. Final elongation took place at $72^{\circ} \mathrm{C}$ for $10 \mathrm{~min}$. For Usp22 $2^{\operatorname{lox} P}$ mice, three separate PCR reactions were run in order to determine their Usp22, Apc and VillinCre ${ }^{\mathrm{ERT} 2}$ status. For genotyping PCR samples were pre-heated to $95^{\circ} \mathrm{C}$ for $3 \mathrm{~min}$. The respective DNA fragments were amplified in repeated polymerization cycles and it was allowed for a final elongation at $72^{\circ} \mathrm{C}$ for $10 \mathrm{~min}$. The respective polymerization cycles for Usp22 were: $95^{\circ} \mathrm{C}$ for $30 \mathrm{~s}, 67^{\circ} \mathrm{C}$ for $30 \mathrm{~s}, 72^{\circ} \mathrm{C}$ for $1 \mathrm{~min}\left(35\right.$ cycles); for $\mathrm{APC}^{1638 \mathrm{~N}}: 95^{\circ} \mathrm{C}$ for $30 \mathrm{~s}, 58^{\circ} \mathrm{C}$ for $30 \mathrm{~s}, 72^{\circ} \mathrm{C}$ for $1 \mathrm{~min}\left(40\right.$ cycles); and for Villin-Cre ${ }^{\mathrm{ERT} 2}: 95^{\circ} \mathrm{C}$ for $15 \mathrm{~s}, 56^{\circ} \mathrm{C}$ for $15 \mathrm{~s}, 72^{\circ} \mathrm{C}$ for $30 \mathrm{~s}(35$ cycles). 


\subsubsection{RNA isolation}

Tissue or cell material was homogenized in TRIzol ${ }^{\circledR}$ and RNA was extracted according to the manufacturer's manual. Briefly, mouse tissues were homogenized in $750 \mu \mathrm{l} \mathrm{TRIzol^{ \circledR }}$ or cell pellets were resuspended in $500 \mu \mathrm{T} \mathrm{TRIzol}{ }^{\circledR}$ and stored at $-20^{\circ} \mathrm{C}$ overnight. $100 \mu \mathrm{l}$ chloroform was added and samples were vortexed for $15 \mathrm{sec}$. Samples were spun down at 10,000 rpm and $4^{\circ} \mathrm{C}$ for $20 \mathrm{~min}$ and the aqueous phase was mixed with an equal volume of isopropanol. After storing samples at $-20^{\circ} \mathrm{C}$ overnight, samples were spun down and the RNA pellet was washed in $80 \% \mathrm{EtOH}$ in DEPC water. After a centrifugation step, the pellets were air-dried at room temperature and resuspended in $30 \mu \mathrm{I}$ DEPC water.

\subsubsection{RNA gel electrophoresis}

To assess RNA integrity, RNA samples (250-500 ng) were mixed with RNA loading dye 1:10 in a total volume of $10 \mu \mathrm{l}$. Samples were applied onto a $1 \%$ agarose gel prepared with TBE buffer and run at $100 \mathrm{~V}$ for $15 \mathrm{~min}$. RNA integrity was assessed based on the intensity of $18 \mathrm{~S}$ rRNA (approximately $2 \mathrm{~kb}$ ) and 28S rRNA (approximately $5 \mathrm{~kb}$; should be more intense than $18 \mathrm{~S}$ rRNA).

\subsubsection{5 cDNA synthesis}

In a total volume of $16 \mu \mathrm{l} 1 \mu \mathrm{g}$ of total RNA was mixed with DEPC water, $2 \mu \mathrm{l}$ of $15 \mu \mathrm{M}$ random primers, $4 \mu \mathrm{dNTPs}$ (each $2.5 \mathrm{mM}$ ) and incubated at $70^{\circ} \mathrm{C}$ for $5 \mathrm{~min}$. After cooling the samples on ice $4 \mu \mathrm{l}$ transcription master mix $(2 \mu \mathrm{l} 10 \mathrm{x}$ reaction buffer, $0.25 \mu \mathrm{l}$ [10 U] RNase inhibitor, $0.125 \mu \mathrm{l}$ M-MuLV reverse transcriptase and $1.625 \mu \mathrm{I}$ DEPC water) was added. cDNA synthesis was performed at $42^{\circ} \mathrm{C}$ for $1 \mathrm{~h}$ and terminated at $95^{\circ} \mathrm{C}$ for $5 \mathrm{~min}$. Finally, samples were diluted with DEPC water to a total volume of $200 \mu \mathrm{l}$. 


\subsubsection{Quantitative Real-Time PCR (qPCR)}

To quantify gene expression levels $3 \mu \mathrm{l}$ cDNA was added to a master mix containing $14 \mu \mathrm{l} 2 \mathrm{x}$ qPCR mix, $6.5 \mu \mathrm{l} \mathrm{H}_{2} \mathrm{O}$, and $1.5 \mu \mathrm{l}$ primers. Samples were quantified based on a standard curve prepared from cDNA pools. Initially, cDNA was denatured at $95^{\circ} \mathrm{C}$ for 2 min. Afterwards, amplification took place in 40 polymerization cycles $\left(95^{\circ} \mathrm{C}\right.$ for $15 \mathrm{~s}, 60^{\circ} \mathrm{C}$ for $\left.1 \mathrm{~min}\right)$ and SYBR Green I detection took place during a melting curve analysis from $60^{\circ} \mathrm{C}$ to $95^{\circ} \mathrm{C}$ with one read every $0.5^{\circ} \mathrm{C}$. Human samples were normalized to the housekeeping gene $18 S \operatorname{rRN} A$ and murine samples to $36 B 4$.

\subsubsection{Luciferase reporter assay}

In order to determine whether the knockdown of USP22 has an effect on Wnt signaling in vitro, a luciferase reporter assay was performed as previously described (Hossan et al., 2016). For this purpose, 45,000 SW480 and HCT116 cells were seeded per well in triplicates into 24-well plates. siRNA-transfections were carried out using Lipofectamine ${ }^{\circledR}$ RNAiMAX according to the manufacturer's protocol. Two days after the knockdown, cells were transfected with SuperTOP/FOP-FLASH (Veeman et al., 2003) plasmids encoding mutated or wild type TCF/LEF binding sites and a firefly- (Photinus pyralis) luciferase reporter construct. In addition, a Renillareporter (Renilla reniformis) was used as a control. Plasmids were complexed with Lipofectamine ${ }^{\circledR} 2000$ for $25 \mathrm{~min}$ at room temperature and added to the wells containing antibiotics-free medium. $24 \mathrm{~h}$ after transfection, cells were washed twice with ice-cold PBS and $250 \mu \mathrm{l}$ Passive Lysis Buffer was added per well. After shaking at room temperature for $15 \mathrm{~min}$, plates were spun down at maximum speed for $1 \mathrm{~min}$ and $50 \mu \mathrm{l}$ of the supernatant was transferred into 96-well Optiplates. All samples were pipetted in triplicates. The luminometer was equilibrated with firefly and Renilla buffer. First, chemiluminescence was measured after adding $100 \mu \mathrm{l}$ firefly luciferase solution to the cell, afterwards the same volume of Renilla 
reagent was measured. For analysis, the average value per triplicate was determined and the ratio of the firefly and Renilla signals was calculated to normalize all samples.

\subsubsection{Protein biochemistry}

\subsubsection{Protein isolation}

To isolate proteins, cell pellets or snap-frozen intestines were lysed in ice-cold RIPA buffer containing $10 \mathrm{mM} \mathrm{N}$-ethylmaleimide, $10 \mathrm{mM}$ Pefabloc, $1 \mathrm{mM}$ Aprotinin/Leupeptin, $0.1 \mathrm{mM}$ lodoacetamide and $10 \mathrm{mM} \beta$-Glycerolphosphate disodium salt hydrate on ice. Afterwards samples were sonicated for 10 min to shear genomic DNA.

\subsubsection{Bicinchoninic acid (BCA) assay}

To determine protein concentrations $\mathrm{BCA}$ assay was performed using the Pierce ${ }^{\mathrm{TM}} \mathrm{BCA}$ Protein Assay Kit. Solution A and B were mixed in a ratio of $50: 1$. Subsequently, $5 \mu$ l protein sample or BSA standard were added and incubated at $37^{\circ} \mathrm{C}$ for $30 \mathrm{~min}$. Protein concentrations were determined spectrophotometrically based on a standard curve.

\subsubsection{SDS-PAGE and western blot}

To prepare the samples for SDS-PAGE, Laemmli buffer was diluted with the protein lysates in a ratio of $1: 5$ and boiled at $95^{\circ} \mathrm{C}$ for $5 \mathrm{~min}$. Proteins were separated by SDS-PAGE at $100-200$ $\mathrm{V}$ and transferred onto a nitrocellulose membrane at $100 \mathrm{~V}$ for $90 \mathrm{~min}$. In order to prevent unspecific binding of antibodies, membranes were blocked in 5\% milk powder in TBS-T for 30$60 \mathrm{~min}$. Proteins were detected using specific primary antibodies and horseradish peroxidaseconjugated secondary antibodies and subsequently visualized using the ChemiDoc ${ }^{\mathrm{TM}}$ MP System. 


\subsubsection{Co-Immunoprecipitation (Co-IP)}

Co-IP was performed to detect the direct interaction between USP22 and HSP90. Protein G Sepharose (PGS) beads were equilibrated by washing them three times with $\mathrm{H}_{2} \mathrm{O}$ (spun down at $300 \mathrm{rpm}, 1 \mathrm{~min})$, and three times with E1A buffer containing protease inhibitors (10 $\mathrm{mM} \mathrm{N}$ ethylmaleimide, $10 \mathrm{mM}$ Pefabloc, $1 \mathrm{mM}$ Aprotinin/Leupeptin, $0.1 \mathrm{mM}$ lodoacetamide and 10 mM $\beta$-Glycerophosphate disodium salt hydrate). To prevent unspecific DNA binding, beads were resuspended in buffer containing salmon sperm DNA in a dilution of 1:10. Beads were incubated on a rotator at $4^{\circ} \mathrm{C}$ for $1 \mathrm{~h}$. To isolate protein $500 \mu \mathrm{E} 1 \mathrm{~A}$ buffer was added per 10 cm plate and HCT116 cells were scraped off. Samples were sonicated for $10 \mathrm{~min}$. Next, beads were washed 5 times in E1A containing inhibitors and 1:5 of the beads was added to the protein sample to clear the lysate at $4^{\circ} \mathrm{C}$ overnight. The residual beads in $\mathrm{E} 1 \mathrm{~A}$ were divided into four portions to conjugate them with $2 \mu \mathrm{g}$ of the respective antibodies: (1) USP22 antibody, (2) HSP90AB1 antibody, (3) non-specific IgG antibody as a background control and (4) only E1A buffer as a negative control. Lysate clearing and antibody binding was performed at $4^{\circ} \mathrm{C}$ overnight. Samples were spun down and the antibody-conjugated beads and the control were washed 5 times with E1A. The protein lysate was added in equal portions to the beads and one part was boiled in $6 x$ Laemmli buffer as an input control. For protein binding to the antibodies samples were incubated further on a rotating device at $4^{\circ} \mathrm{C}$ overnight. Beads were washed $5 x$ with E1A and an equal volume of 2x Laemmli buffer was added. Samples were boiled at $95^{\circ} \mathrm{C}$ for $5 \mathrm{~min}$ and stored at $-20^{\circ} \mathrm{C}$ or directly used for western blot analysis.

\subsubsection{Next generation sequencing}

\subsubsection{Microarray using mouse embryonic fibroblasts}

To generate global gene expression data RNA was isolated from MEFs obtained from four wild type and four Usp22 lacz/lacz animals. Quality control of RNA samples as well as microarray analyses were performed by the Transcriptome Analysis Laboratory (TAL) Göttingen, 
Germany. Briefly, the integrity of the input RNA was analyzed using an Agilent BioAnalyzer. $200 \mathrm{ng}$ of total RNA was reverse transcribed into cDNA which was labelled by a T7 RNA Polymerase incorporating cyanine 3-CTP, fluorescent nucleotide analogs. These Cy3-CTPlabelled cRNA samples were fragmentized and hybridized to $4 \times 44 \mathrm{~K}$ microarray slides at $65^{\circ} \mathrm{C}$ for approximately 17 hours according to the manufacturer's protocol. Arrays were washed with a $0.005 \%$ Triton X-102-containing buffer to reduce the presence of artifacts. Cy3-fluorescence of complementary bound probes on the microarray slides was detected and fluorescence intensities were quantified and compared amongst samples.

\subsubsection{2 mRNA Library Preparation}

Prior to preparing libraries for subsequent mRNA-seq analysis, integrity was confirmed using the Bioanalyzer system comparing the ratio of $18 \mathrm{~S}$ and $28 \mathrm{~S}$ rRNA fragments. Afterwards, $1 \mu \mathrm{g}$ RNA was processed with the NEXTflex ${ }^{\text {TM }}$ Rapid Illumina Directional RNA-Seq Library Prep Kit. Briefly, mRNA was purified by Poly $(A)$ magnetic beads and fragmented. First and second strand cDNA synthesis was performed and single base overhangs were prepared and adapters were ligated to the cDNA. This product was amplified using specific barcode-containing primers allowing the subsequent identification of samples. Finally, DNA concentration was determined by Qubit ${ }^{\circledast}$ Fluorometer measurement. 16 samples were pooled to a final concentration of $2 \mathrm{nM}$. Sequencing was performed by the TAL using Illumina ${ }^{\circledR}$ HiSeq 2000 sequencers.

\subsubsection{3 mRNA-seq data processing}

FASTQ files obtained from the TAL were mapped to the human reference genome (assembly hg19) by Bowtie2 using default parameters. The number of reads per sample was determined using HTSeq. Subsequently, data were normalized and processed via DESeq2 on the statistical software R. Similarities between replicates have been determined using hierarchical clustering and PCA plots. For analysis of differentially expressed genes among siControl and 
siUsp22 samples, following thresholds were defined: BaseMean $\geq 10$, log2fold change \pm 0.8 , padj $\leq 0.05$. Heatmaps were generated using $\mathrm{R}$ or Morpheus. Gene Ontology analysis was performed with the aforementioned threshold values using GOTermFinder and were subsequently visualized using REViGO.

\subsubsection{Statistical analyses}

All graphs in this study have been designed with GraphPad Prism version 5.04 (GraphPad Software, Inc.). P-values were determined using Student's $t$-test $\left({ }^{* *} p \leq 0.001,{ }^{* *} p \leq 0.01,{ }^{*} p \leq\right.$ 0.05.). 


\section{Results}

\subsection{USP22 is required for proper murine development and lineage specification}

While previous studies defined several deubiquitination and interaction partners of USP22, its general role in in vivo remained unclear. Initially, to unravel the general function of USP22 in mice, we aimed to determine its sites of expression and to investigate its relevance during organ maintenance. Consequently, to investigate both the expression pattern and function of Usp22 in vivo, we generated the so-called Usp22lacz mice. This mouse line has been previously characterized in the aforementioned M.Sc. thesis (Kosinsky, 2013) and has recently been described by our group (Kosinsky et al., 2015). In the current project we increased the size of the experimental mouse cohort in order to confirm previous observations and to identify further Usp22-related cellular processes. To determine the expression levels of Usp22 and therefore determine the knockout efficiency, brain and small intestines (SIs) were isolated from 4 monthold mice. Quantitative RT-PCR (qRT-PCR) analysis revealed a significant reduction of Usp22 expression in both organs; however, approximately $3 \%$ of residual correctly spliced mRNA was still detectable in mutant animals compared to their wild type littermates (Figure 10A). Moreover, an incomplete reduction of USP22 protein levels was observed in western blot (Figure 10B). Interestingly, the homozygous expression of this hypomorphic Usp22 lacz allele led to a clear growth defect in adult animals at an age of four months; a phenotype which could be maintained even after several generations of breedings (Figure 10C). Accordingly, the body weight of Usp22laczlacz mice was significantly reduced as revealed after recording the weight of adult animals, as here indicated for males (Figure 10D). In contrast, in heterozygous animals the body size and weight were only marginally affected. These findings support our previous observations that Usp22-hypomorphic mice are viable but are affected by growth retardation. 
A

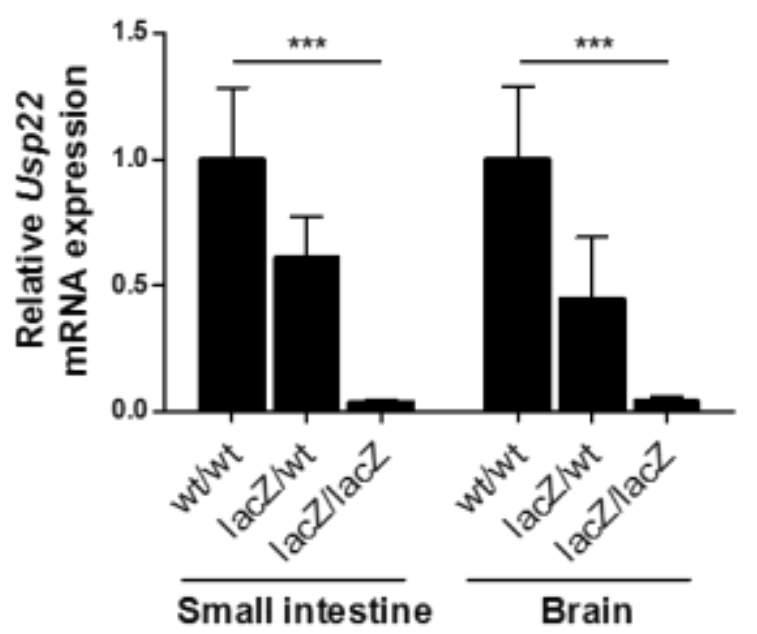

C

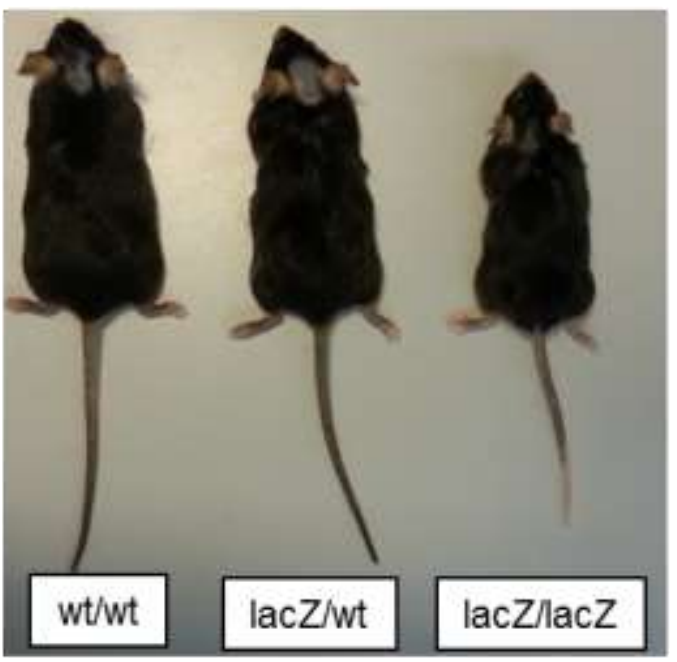

B

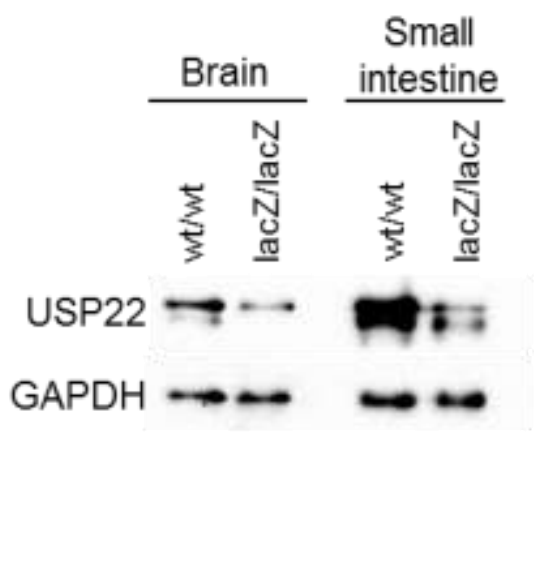

D

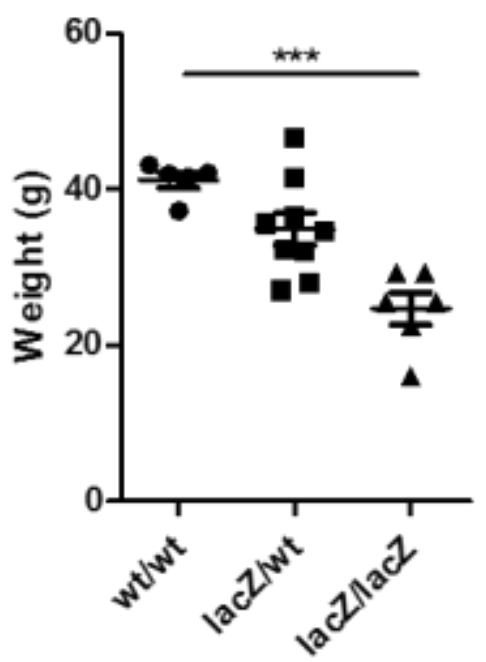

Figure 10: Generation and phenotype of Usp22-hypomorphic mice. (A) The reduction of Usp22 on mRNA and (B) protein level was shown using small intestine and brain material. Mean \pm SEM, Student's $t$-test, Usp22 wtwt: $n=4$; Usp22lacz/wt: $n=5$; Usp22laczlacz: $n=4$. (C) Decreased Usp22 expression results in growth retardation and (D) lower body weight as shown for 4-month old male mice. Mean \pm SEM, Student's $t$-test, Usp22 $2^{\text {thtwt: }} n=5$; Usp22lacZ/wt: $n=9$; Usp22laczlacz: $n=6$ (Kosinsky et al., 2015).

In order to understand the function and the mechanism underlying the phenotype of Usp22hypomorphic mice, it is crucial to identify tissues and cell types in which Usp22 is expressed. For this purpose, we took advantage of the LacZ reporter gene under control of the endogenous Usp22 promoter to determine its spatio-temporal expression pattern during early and late stages of embryonic development. So far, the presence of Usp22 expression has only 
been analyzed in whole tissue lysates or during very early stages of embryonic development, i.e. E10.5 and E12.5 (Lee et al., 2006). Beta-galactosidase enzymatic activity and therefore endogenous Usp22-promoter activity was visualized in whole mount stainings when the substrate X-gal was cleaved and thereby a blue staining was produced. At E10.5 Usp22laczlacz embryos showed strong color development in the skin and also blue staining was detected in the brain, spinal cord and intestines (Figure 11A). In order to obtain a better overview of the inner organs, we investigated Usp22 expression sites in later stages of development using E15.5 embryos. We detected strong staining of the skin in Usp22 $2^{\text {lacz/wt }}$ and Usp22 $2^{\text {lacZlacz }}$ animals, however, inner organs were not visible through the skin (Figure 11B). To overcome this problem embryos were cut sagittally and stained a second time for $\beta$-galactosidase activity (Figure 11C). Afterwards, paraffin sections were prepared and counterstained. Besides in the skin, strong staining was detected in connective and muscle tissue (Figure 11D, Table 15). In addition, the facial region including tongue, lips and nasal cavity as well as the brain (i.e. frontal lobe, cerebral cortex, subventricular zone and ganglionic eminences) were stained. Moreover, Usp22 promoter activity was visualized in the heart, lung, kidneys, penis, thymus, bladder, pancreas, thyroid and intestinal system. In summary, X-gal staining revealed ubiquitous Usp22 expression suggesting its relevance during the development of embryonic tissues. 

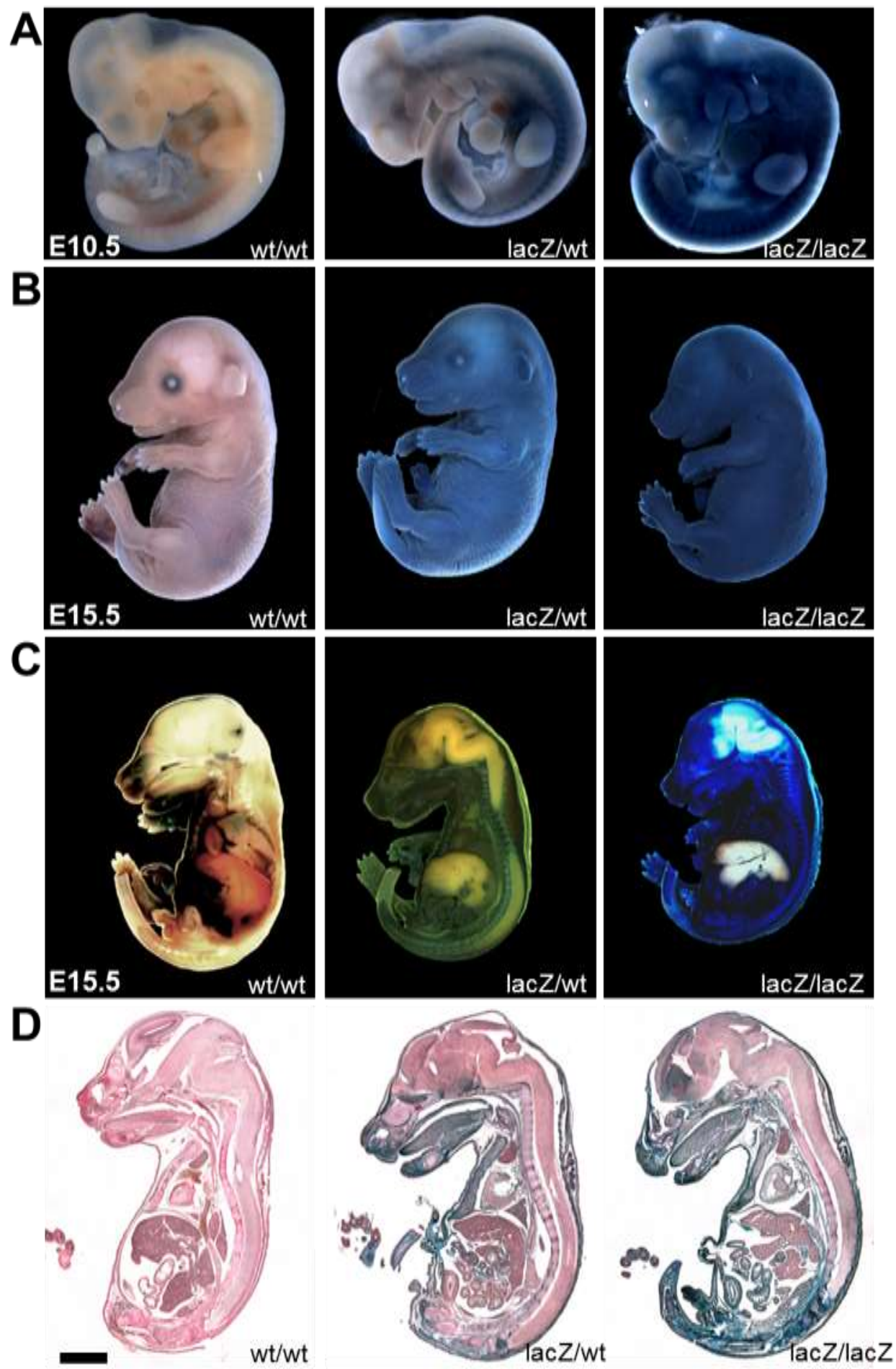

Figure 11: Ubiquitous expression of Usp22 in murine embryos. Using X-Gal staining $\beta$ galactosidase activity, and therefore Usp22 expression, was detected in Usp22 ${ }^{\text {lacz }}$ embryos at (A) E10.5 and (B) E15.5. At E10.5 strong staining of the skin, the spinal cord and inner organs was detected. (C) To observe staining intensity in the inner body regions at E15.5, embryos were cut sagittally and stained again. (D) Sections were prepared and ubiquitous Usp22 expression was detected. Scale bar: 2,000 $\mu \mathrm{m}$ (Kosinsky et al., 2015). 
Table 15: Usp22 levels visualized by X-gal staining in embryonic tissues at E15.5 (Kosinsky et al., 2015).

\begin{tabular}{|c|c|c|c|}
\hline Organ & Staining intensity & Organ & Staining intensity \\
\hline Front lobe & +++ & Thyroid & ++ \\
\hline Cerebellum & + & Heart & ++ \\
\hline Cerebral cortex & ++ & Thymus & ++ \\
\hline Subventricular zone & + & Lung & +++ \\
\hline Midbrain & + & Liver & - \\
\hline Hindbrain & + & Intestinal system & +++ \\
\hline Ganglionic eminences & ++ & Pancreas & ++ \\
\hline Spinal cord & + & Kidneys & +++ \\
\hline Nasal cavity & +++ & Bladder & +++ \\
\hline Lips & +++ & Penis & +++ \\
\hline Tongue & +++ & & \\
\hline
\end{tabular}

As the previous results pointed at an involvement of Usp22 in embryo and tissue development, we next investigated the effect of Usp22 expression on organ maintenance in adult mice. For this purpose, the gross morphology of small intestines isolated from 4-month old animals was evaluated. Swiss rolls were prepared, fixed in formaldehyde and embedded in paraffin. After staining sections with $\mathrm{H} \& \mathrm{E}$, we assessed proximal, intermediate and distal organ segments (Figure 12A). Generally, the number of crypts and villi was not affected by the reduction of Usp22 levels (data not shown). Moreover, we counted 22 to 24 cells per crypt in all mice by analyzing at least 50 crypts per animal (Figure 12B). In addition, we have measured villus length which is representative for the approximate cell numbers. In all genotypes, the average length per villus was between $264 \mu \mathrm{m}$ and $266 \mu \mathrm{m}$ (Figure 12C). In summary, low Usp22 expression levels do not change the gross morphology of the small intestine. 
A
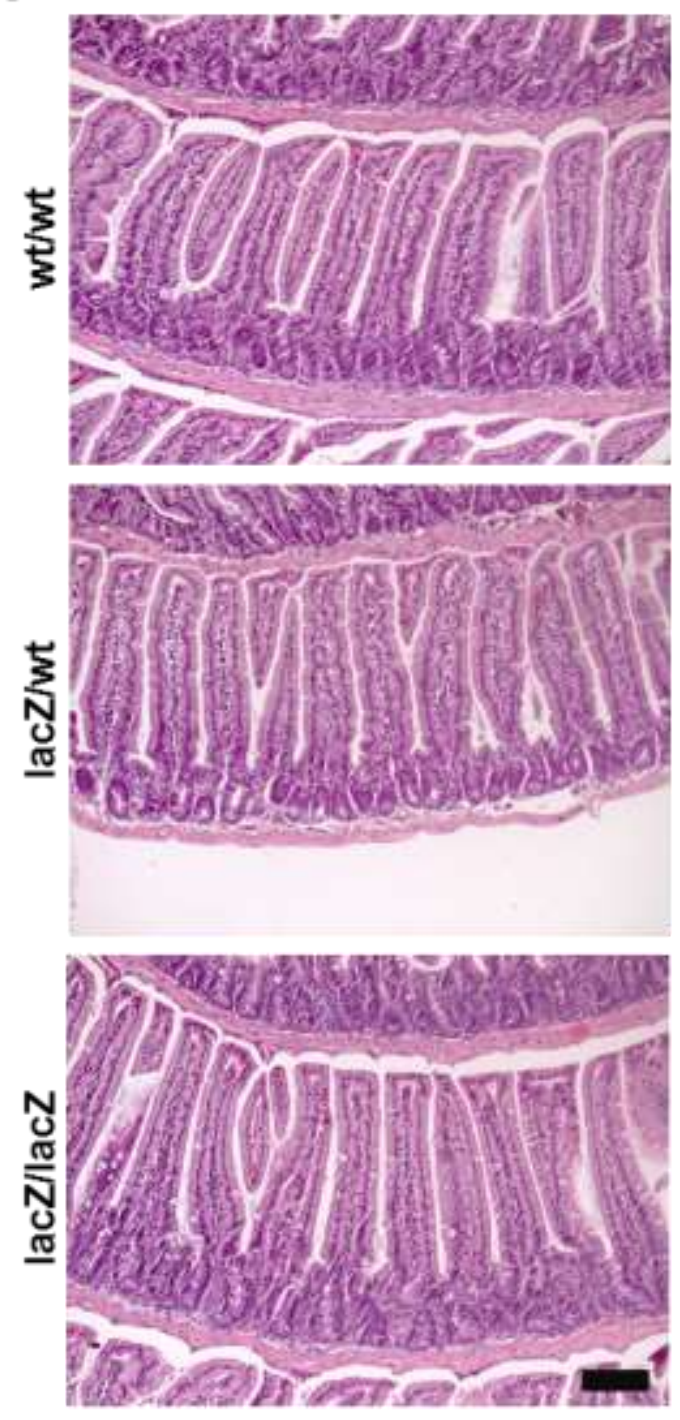

B
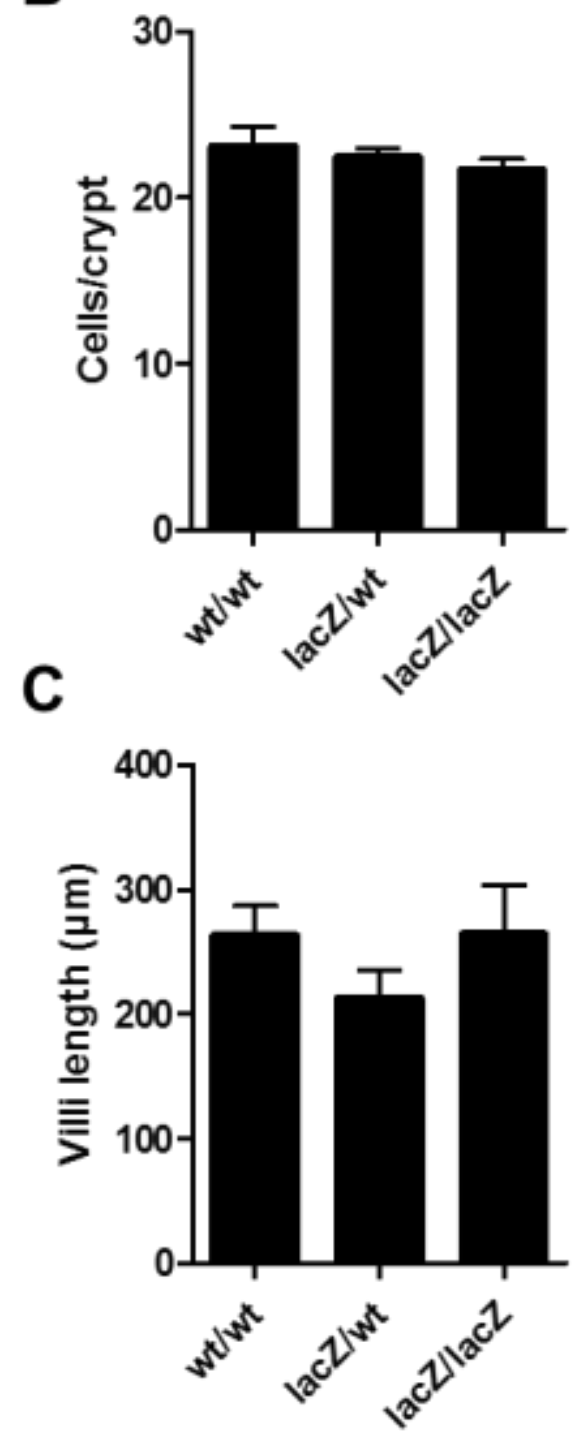

Figure 12: Reduced Usp22 levels do not alter small intestine morphology. (A) Small intestine sections were stained with H\&E to evaluate the gross organ morphology. Scale bar: $100 \mu \mathrm{m}$. (B) The number of cells per crypt and the (C) villi length were not changed in Usp22hypomorphic mice. Mean \pm SEM, Student's $t$-test, Usp22 ${ }^{\text {wtwt }}: n=3$; Usp22lacZ/wt: $n=3$; Usp22lacZlacz: $n=3$ (Kosinsky et al., 2015).

As described above, we have previously observed that the differentiation pattern in the small intestine is affected in Usp22laczlacz mice (Kosinsky, 2013; Kosinsky et al., 2015). We identified a slight increase in the Olfm4-positive stem cells and accordingly more differentiated cells, i.e. Goblet, Paneth and enteroendocrine cells. To test the effect of Usp22 loss in another organ 
system, analyses of the brain were performed in cooperation with Dr. Nicole Hellbach and Prof.

Dr. Tanja Vogel (Department of Molecular Embryology, Institute of Anatomy and Cell Biology, Faculty of Medicine, University of Freiburg, Germany).

A
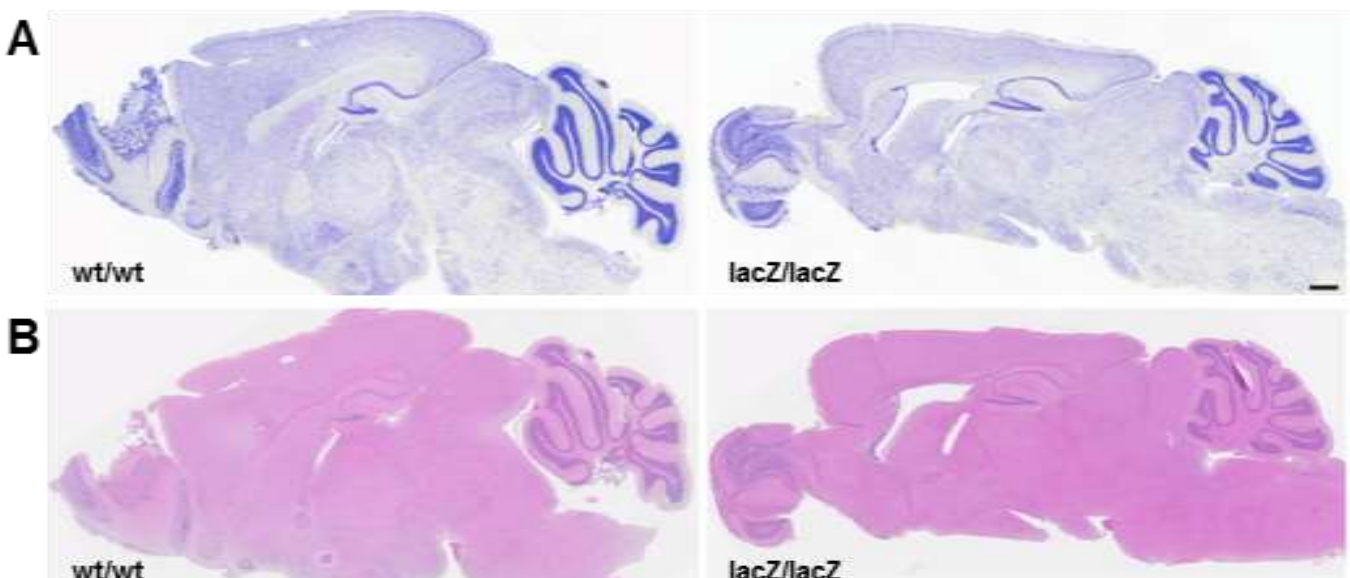

lacZllacZ
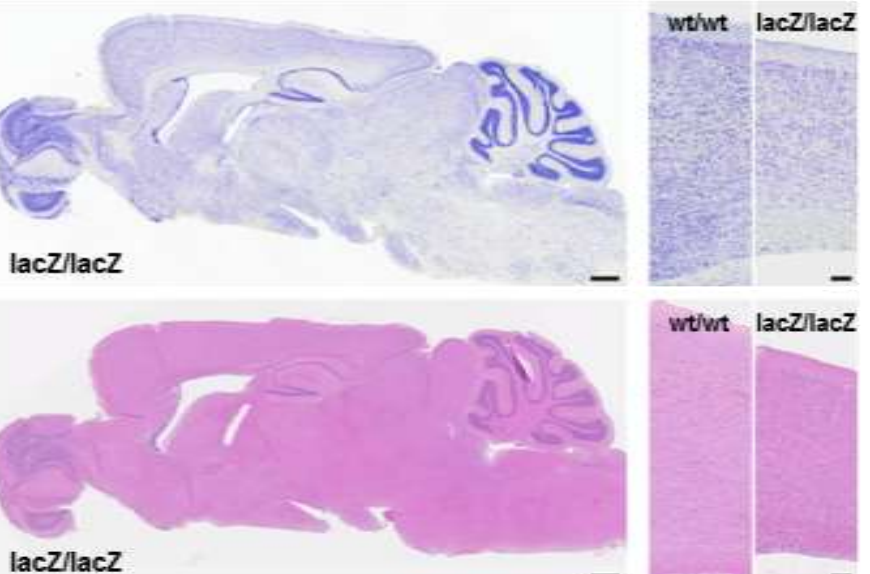

witivit laczlacz
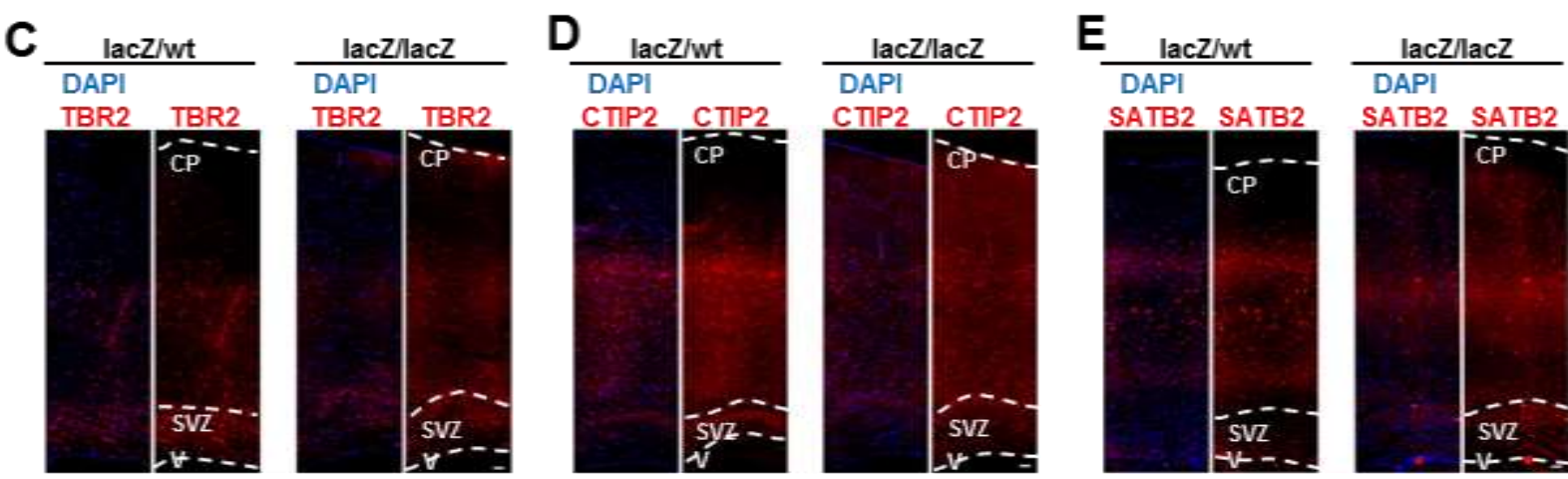

Figure 13: Usp22 affects cortical differentiation in the adult mouse brain. (A) Nissl and (B) H\&E staining on sagittal brain sections revealed a less densely packed cortex in adult Usp22lacZllacZ mice. Scale bar: $500 \mu \mathrm{m}$. (C-E) By IHC progenitor and differentiation markers were visualized on coronal cortex sections. While the distribution of TBR2-positive intermediate precursor cells in the subventricular zone was unchanged, the density of deep-layer (CTIP2) and upper-layer (SATB2) neurons was decreased (Kosinsky et al., 2015). Scale bar: $100 \mu \mathrm{m}$. CP: cortical plate; SVZ: subventricular zone; V: ventricle.

To evaluate the general brain morphology, $\mathrm{H} \& \mathrm{E}$ and Nissl stainings were performed. While the gross brain morphology was unaffected, the total organ size was reduced in Usp22 lacz/lacz mice (Figure 13A, B). Moreover, the cell density in the cerebral cortex was lower in these animals. To investigate potential differentiation shifts, the number of progenitors and differentiated cells was observed in the subventricular zone as well as deep-layer and upper-layer neurons using 
immunohistochemistry. Intermediate precursor cells (IPCs) were visualized by staining for TBox Transcription Factor 2 (TBR2) in the subventricular zone. We observed that the localization and number of IPCs was not significantly affected after the reduction of Usp22 levels (Figure 13C). Next, differentiated early born deep-layer neurons and later born upper-layer neurons were detected by staining for C2H2-Type Zinc Finger Protein (CTIP2) and AT-Rich SequenceBinding Protein 2 (SATB2), respectively (Figure 13D, E). Interestingly, in Usp22 laczlacz mice the density of CTIP2- and SATB2-positive cells was decreased. These results support our observations in the small intestine indicating that Usp22 affects lineage specification and differentiation in diverse organ systems.

As reported previously, USP22 is able to deubiquitinate the histone H2B and thereby affect transcription (Zhang et al., 2008b). In addition, our group revealed the significance of H2Bub1 in differentiation processes (Karpiuk et al., 2012). Thus we aimed to investigate whether H2Bub1 levels correlated with the differentiation shifts observed in adult mouse organs. For this purpose we stained small intestine sections for H2B and H2Bub1 using immunohistochemistry. Surprisingly, no difference in the number of positively stained cells, their staining intensity or localization was detected (Figure 14) suggesting that the effects of USP22 on differentiation are independent of its ability to deubiquitinate H2B. 


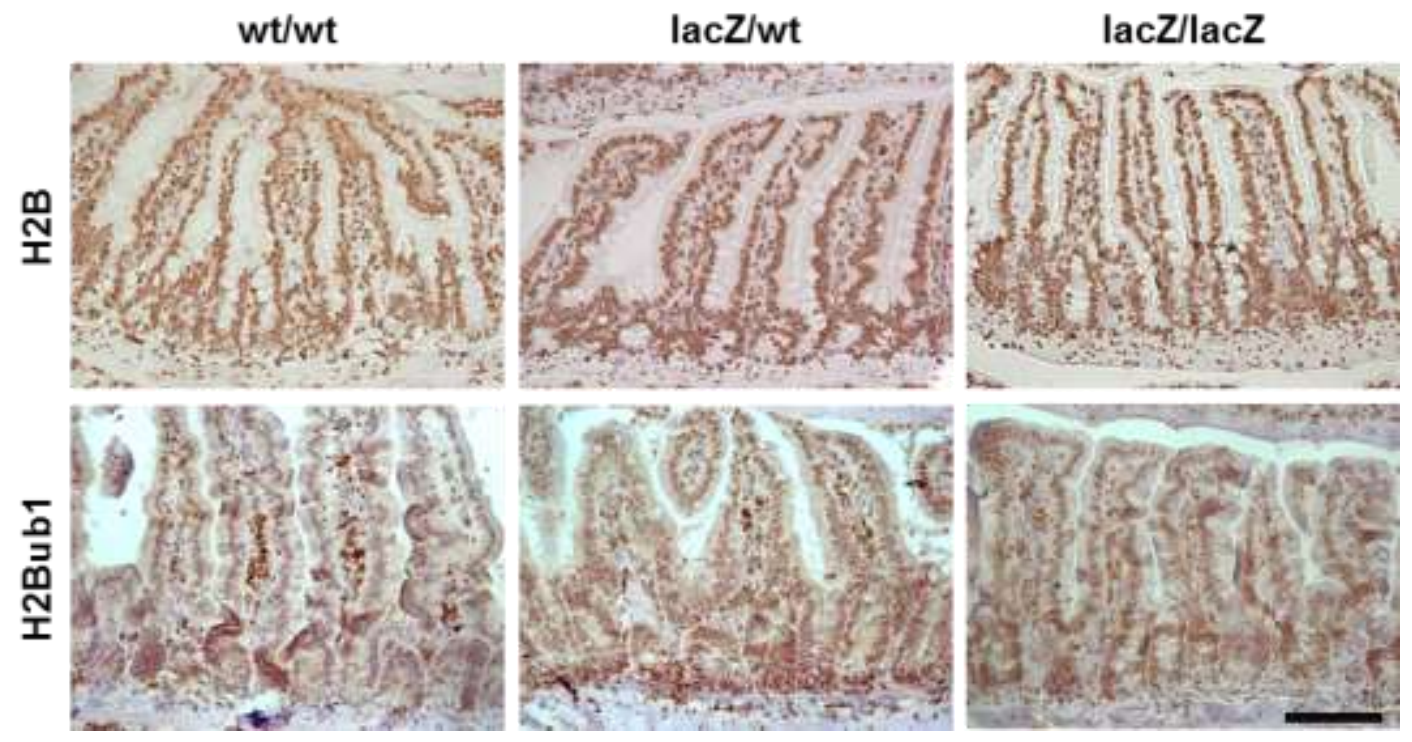

Figure 14: H2B and H2Bub1 levels are not affected by reduced USP22 amounts in the small intestine. $\mathrm{H} 2 \mathrm{~B}$ and $\mathrm{H} 2 \mathrm{Bub} 1$ levels have been visualized by immunohistochemistry on small intestine sections. Reduced USP22 levels did not result in altered staining intensities, numbers of positively stained cells or in changed localization of H2B/H2Bub1-positive cells (Kosinsky et al., 2015). Scale bar: $100 \mu \mathrm{m}$.

To investigate the function of Usp22 in detail, we isolated mouse embryonic fibroblasts (MEFs) from eight individual embryos to perform subsequent gene expression analyses. RNA was isolated from four Usp22 $2^{\text {wt/wt }}$ and four Usp22 lacz/lacz MEF samples and microarray-based profiling was carried out at the Transcriptome and Genome Analysis Laboratory (TAL), Göttingen, Germany. The variance between the four animals per genotype was assessed by hierarchical clustering and replicates were compared in a heatmap. As expected, the variance between the individual animals was rather high (Figure 15A). While Usp22 ${ }^{\text {laczlacz }}$ samples clustered together, there was a relatively high variation among wild type samples. Furthermore, we compared the variance in differentially regulated genes with high fold changes (log2fold change \pm 0.8 , padj $<0.05)$ using the Morpheus tool. Despite the evident heterogeneity among the replicates, several differentially expressed genes were identified as depicted in the heatmap (Figure 15B). 
A

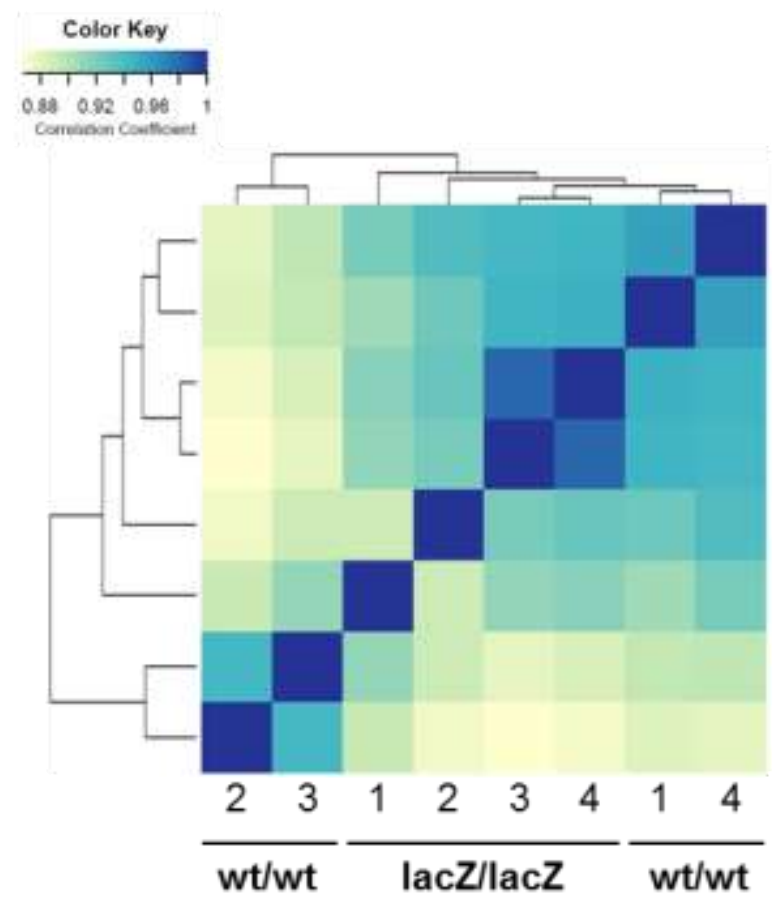

B
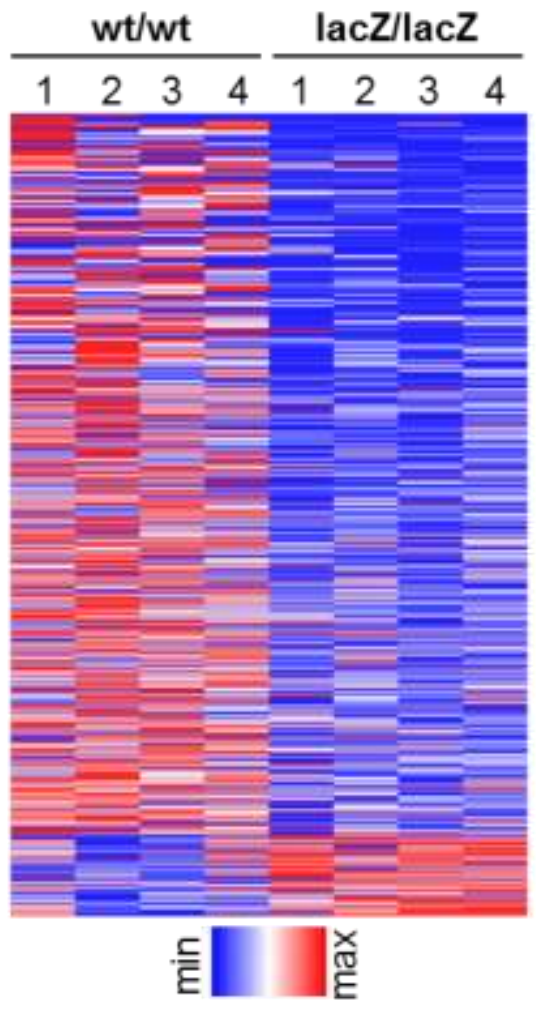

Figure 15: Quality control of MEFs used for microarray analysis. (A) Hierarchical clustering of replicates reveals the heterogeneity of murine material. (B) Heatmap generating with the Morpheus tool displaying all replicates and their expression for genes with a high fold change (log2fold change \pm 0.8 , padj $<0.05$ ). Each row represents a single gene. Expression varies between replicates but the overall tendency was similar. The color code indicates the relative minimum (min) and maximum (max) expression value per gene.

To investigate biological processes affected by the loss of Usp22 in MEFs, microarray data were subjected to gene ontology (GO) analysis, i.e. by GOTermFinder (Princeton University, Lewis-Sigler Institute) and the GO visualization tool REViGO (Supek et al., 2011). As expected, there was a significant enrichment in GO terms involved in developmental processes, proliferation and differentiation (Figure 16, Table 16). These findings support our in vivo data

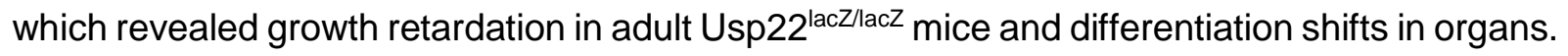




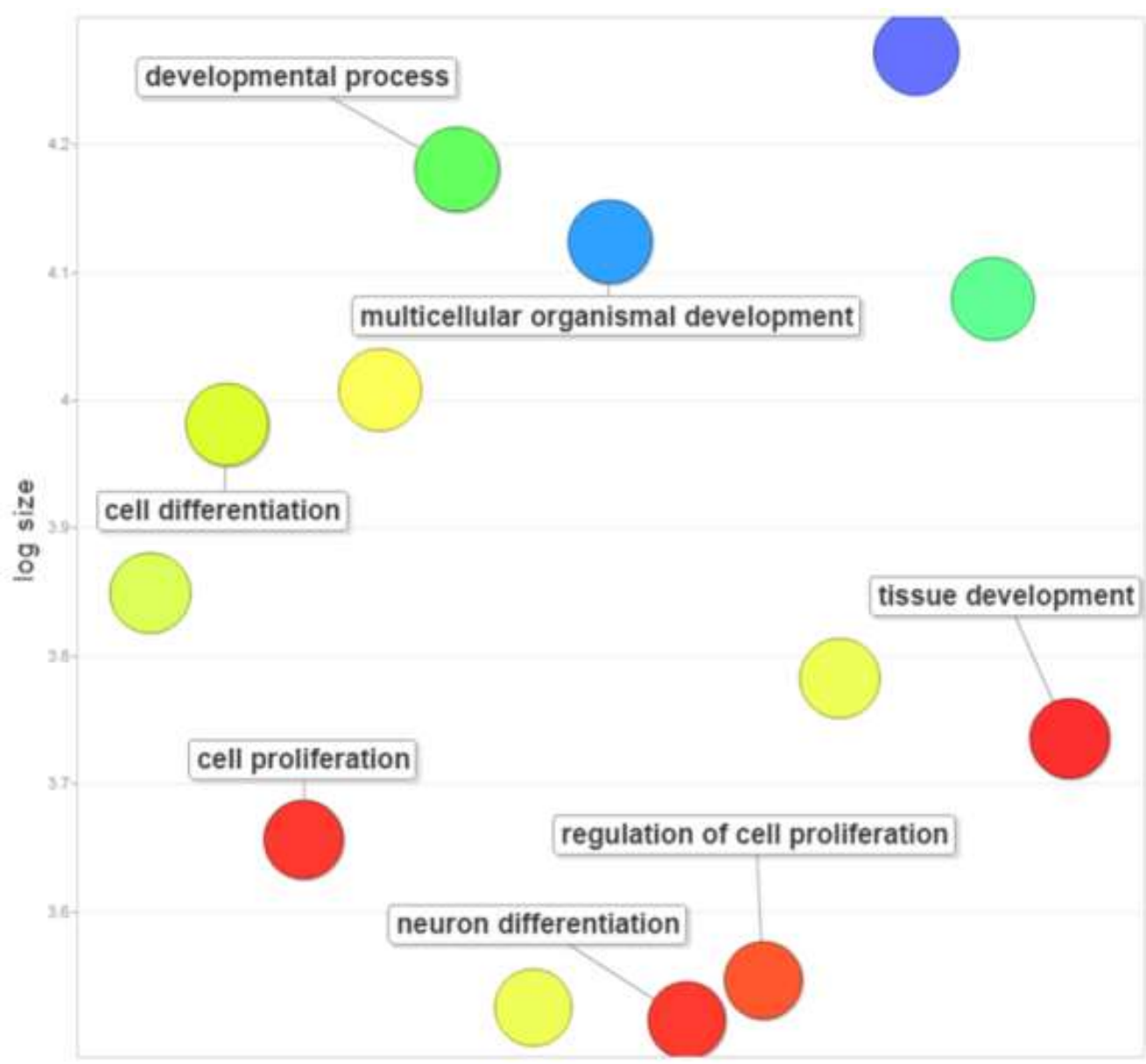

Order: Alphabetical

Figure 16: Gene Ontology analysis of genes affected by reduced Usp22 expression in MEFs. Summary of enriched GO terms after analyzing differential regulated genes (log2fold change \pm 0.8 , p-value $<0.05$ ) in Usp22laczlacz MEFs using the GO visualization tool REViGO. Analysis revealed association with development-, proliferation- and differentiation-associated processes.

In summary, the Usp22 lacz mouse line enabled us to investigate the function of Usp22 in murine development and organ maintenance. Staining for $\beta$-galactosidase activity revealed ubiquitous Usp22 expression in the majority of murine embryonic tissues. As a consequence, reduced Usp22 levels result in systemic repercussions in adult mice, i.e. growth retardation and consistently reduced body weight. While the morphology of the small intestine and brain were only marginally changed, the cell differentiation pattern was altered in these organs. Interestingly, these phenomena seem to be independent of H2B monoubiquitination. Using 
microarray-based gene expression and GO analyses in MEFs, we were able to confirm the involvement of Usp22 in developmental and differentiation processes.

Table 16: GO terms enriched in Usp22 ${ }^{\text {lacz/lacz }}$ MEFs as revealed by GOTermFinder.

\begin{tabular}{|c|c|c|c|c|}
\hline Term ID & Gene Ontology term & $\begin{array}{l}\text { Cluster } \\
\text { frequency }\end{array}$ & $\begin{array}{l}\text { Corrected } \\
\text { p-value }\end{array}$ & FDR \\
\hline GO:0032502 & Developmental process & $36.9 \%$ & 0.00000846 & 0 \\
\hline GO:0044767 & Single-organism developmental process & $36.9 \%$ & 0.0000038 & 0 \\
\hline GO:0048856 & Anatomical structure development & $35.9 \%$ & 0.000000982 & 0 \\
\hline GO:0007275 & Multicellular organism development & $33.4 \%$ & 0.000000453 & 0 \\
\hline GO:0048731 & System development & $29.7 \%$ & 0.00000471 & 0 \\
\hline GO:0048869 & Cellular developmental process & $27.8 \%$ & 0.00018 & 0 \\
\hline GO:0030154 & Cell differentiation & $26.5 \%$ & 0.0000724 & 0 \\
\hline GO:0051239 & $\begin{array}{l}\text { Regulation of multicellular organismal } \\
\text { process }\end{array}$ & $20.4 \%$ & 0.00011 & 0 \\
\hline GO:0009653 & Anatomical structure morphogenesis & $20.1 \%$ & 0.0000509 & 0 \\
\hline GO:0048468 & Cell development & $16.0 \%$ & 0.00361 & 0 \\
\hline GO:0008283 & Cell proliferation & $14.3 \%$ & 0.00699 & 0 \\
\hline GO:0009888 & 3Tissue development & $13.8 \%$ & 0.00905 & 0 \\
\hline GO:0042127 & Regulation of cell proliferation & $12.5 \%$ & 0.00334 & 0 \\
\hline GO:0030182 & Neuron differentiation & $11.3 \%$ & 0.00665 & 0 \\
\hline GO:0060429 & Epithelium development & $11.1 \%$ & 0.0001 & 0 \\
\hline
\end{tabular}




\subsection{The role of USP22 in colorectal tumorigenesis in vivo}

After investigating the function of USP22 in organ maintenance and differentiation, we aimed to elucidate the effect of USP22 loss on colorectal cancer formation and progression in vivo. This project was based on the hypothesis that an Usp22 loss lowers tumor burden since it was described that USP22 is overexpressed in CRC and further malignancies. For this purpose, we generated a mouse line which is based on the aforementioned Usp22 lacz animals. The 'knockout first' allele contains FLP recombinase target (FRT) sequences which are recognized by flippase (FLP) recombinase. After crossing Usp22 lacz animals to transgenic FLP mice the lacZ sequence and neomycin-resistance cassette were excised leaving loxP-sites flanking exon 2 of the Usp22 gene (Figure 17A). Thereby the allele was converted to a conditional knockout allele based on the Cre/loxP system restoring Usp22 gene activity. These mice were crossed with Villin-Cre ${ }^{\mathrm{ERT} 2}$ mice in which the Cre-recombinase is fused to a mutated Tamoxifeninducible estrogen receptor ligand binding domain (ERT2) (el Marjou et al., 2004). By intraperitoneal Tamoxifen injections an Usp22 loss can be induced in the small intestine and to a lesser extent in the colon. In order to promote colorectal tumorigenesis mice were crossed with APC ${ }^{1638 \mathrm{~N}}$ mice. This mutation in the tumor suppressor results in a globally truncated APC protein as frequently observed in human colorectal cancers. Thus, for this project we analyzed six genotypes; $\mathrm{APC}^{+/+}$or $\mathrm{APC}^{1638 \mathrm{~N} /+}$ each with Usp22 wild type, heterozygous or knockout status (hereafter referred to as Usp22 $2^{+/+}$, Usp22 $2^{+/-}$or Usp22genotypes, genomic DNA was isolated from tail biopsies. Finally, after using specific primers for conventional PCR, DNA fragment sizes were evaluated (Figure 17B; Usp22 wt: 322 bp, loxP: 470 bp; APC wt: 178 bp, 1638N: 347 bp; Villin positive: $220 \mathrm{bp}$ ). Initially, to determine the knockout efficiency, $\mathrm{APC}^{+/+}$mice were injected with Tamoxifen at an age of 4 weeks for 5 consecutive days and Usp22 mRNA levels were determined 4 weeks later. Using qRT-PCR

we detected a decrease of Usp22 levels by approximately $96 \%$ in intestinal epithelial cells 
(Figure 17C). A corresponding decrease on protein level was confirmed by western blot analysis (Figure 17D).
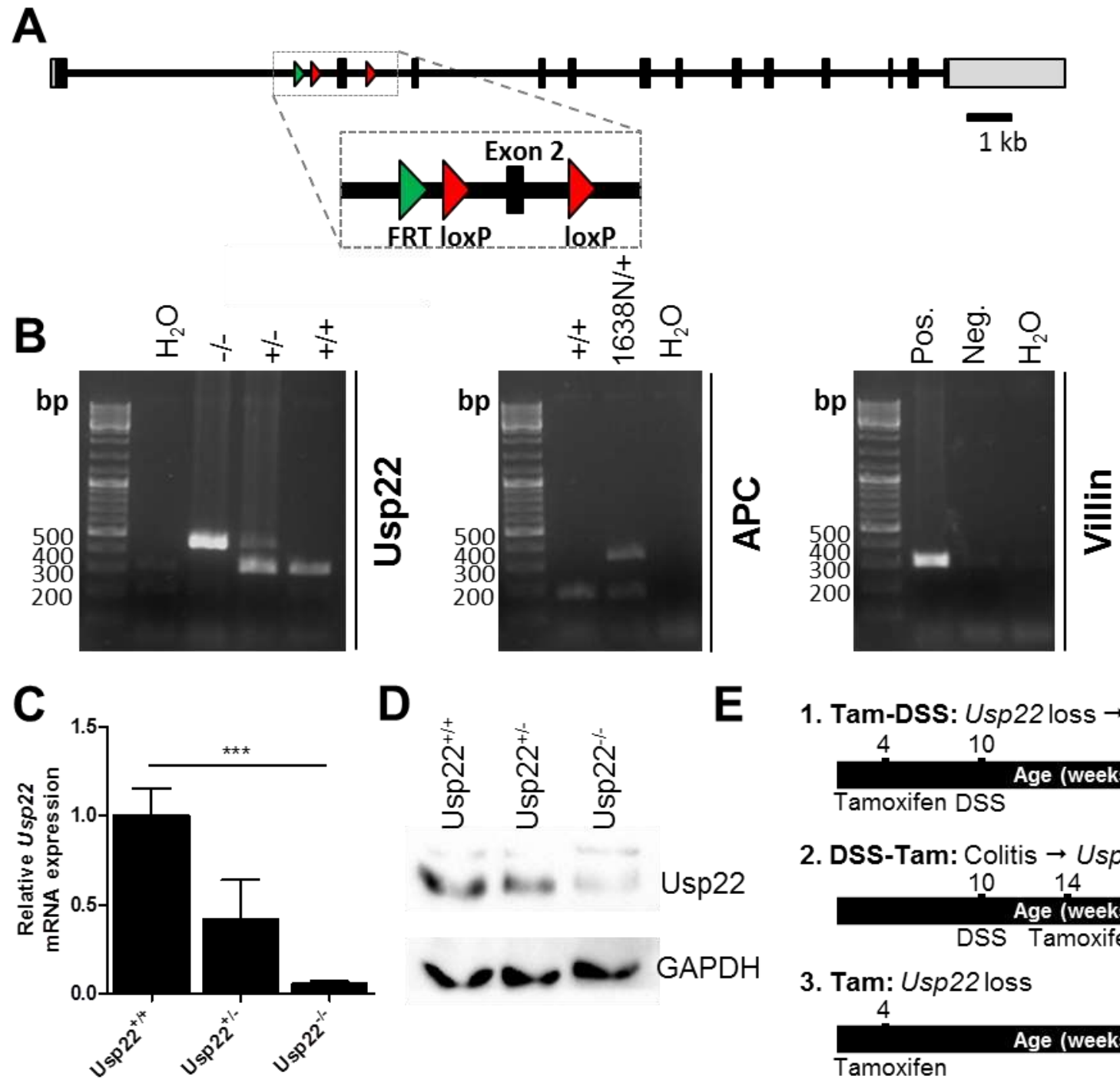

1. Tam-DSS: Usp22 loss $\rightarrow$ Colitis Tamoxifen DSS Age (weeks)

2. DSS-Tam: Colitis $\rightarrow$ Usp22 loss

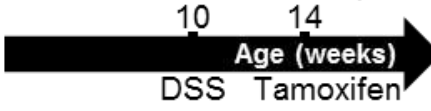

3. Tam: Usp22 loss

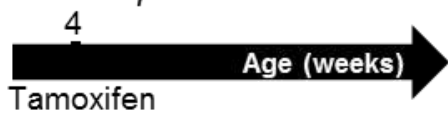

Figure 17: Generation of mice with a conditional intestinal Usp22 knockout. (A) In VillinCre ${ }^{\mathrm{ERT} 2}$ positive, APC ${ }^{1638 \mathrm{~N} /+}$ mice the second exon of the Usp22 gene was flanked by loxPsequences. This allowed an intestine-specific and Tamoxifen-inducible deletion of Usp22. (B) By conventional PCR, genotypes were determined based on DNA fragment sizes (Usp22 wt: 322 bp, loxP: 470 bp; APC wt: 178 bp, 1638N: 347 bp; Villin-Cre ${ }^{\text {ERT2 }}$ positive: 220 bp). (C) Usp22 mRNA levels were reduced by $96 \%$ in Usp22 ${ }^{--}$mice. Mean \pm SEM, Student's $t$-test, $n=5$. (D) Accordingly USP22 protein levels were decreased after Tamoxifen treatment. (E) DSS treatment time points to cause inflammation-induced CRC. Colitis was induced either after (group 1; Tam-DSS) or prior to Usp22 knockout (group 2; DSS-Tam). Additionally, a group for sporadic CRC (group 3; Tam) without DSS treatment has been studied. 
After demonstrating that the knockout efficiency was sufficient, we divided the mice into three experimental cohorts (Figure 17E). The first cohort (Tam-DSS) reflected the situation of colitisinduced colorectal cancer. For this purpose, the knockout of Usp22 was induced and mice were treated with DSS to induce colitis afterwards. In the second experimental group (DSSTam) colitis was induced as well, however, in contrast to the first group, DSS-treatment was performed prior to the loss of Usp22. The last cohort (Tam) was only treated with Tamoxifen and therefore represented a model for sporadic CRC formation. In the following sections these mouse cohorts and the corresponding experimental outcomes will be explained in detail.

Table 17: Mouse numbers per experimental cohort used throughout the study to determine the function of USP22 in colorectal tumorigenesis.

\begin{tabular}{|l|c|c|c|c|c|c|}
\cline { 2 - 7 } \multicolumn{1}{c|}{} & \multicolumn{3}{c|}{ APC $^{+/+}$} & \multicolumn{3}{c|}{ APC $^{1638 \mathrm{~N} /+}$} \\
\cline { 2 - 7 } \multicolumn{1}{c|}{} & Usp22 $^{+/+}$ & Usp22 & \\
\hline Tam-DSS & 8 & 10 & 13 & 16 & 13 & 9 \\
\hline DSS-Tam & 10 & 6 & 5 & 7 & 6 & 4 \\
\hline Tam & 15 & 14 & 8 & 9 & 8 & 7 \\
\hline
\end{tabular}




\subsubsection{Inflammation-induced CRC: Intestinal Usp22 deletion prior to colitis (Tam-DSS)}

\subsubsection{Intestinal Usp22 loss shortens survival and increases intestinal tumor burden}

When starting this project we aimed to utilize a mouse model in which tumorigenesis was not promoted by an APC mutation alone, but also by colitis. For this purpose, experimental mice used to observe inflammation-induced CRC (treatment group 1: Tam-DSS) were injected with Tamoxifen at an age of 4 weeks to induce the intestine-specific Usp22 knockout. When the animals were 10 weeks old and could physically withstand the burden of colitis we started the DSS treatment. We initially administered $2 \%$ of the chemical agent DSS dissolved in drinking water for 7 days. However, we observed strong intestinal bleedings, severe diarrhea and subsequent death in several mice. Therefore, we decided to reduce the load by treating the animals with $2 \%$ DSS for 4 days, feed them with mush food supplemented with glucose for 7 days and continue the DSS treatment for 3 further days. Thus, DSS treatment was terminated when the animals were 12 weeks old. The animal weight, stool consistency and the presence of occult blood was recorded daily from the first day of DSS supplementation until 1 week after terminating the treatment. Despite the reduced burden we observed increased weight loss (Figure 18A), intestinal bleedings (Figure 18B) and severe diarrhea (data not shown) in $\mathrm{APC}^{1638 \mathrm{~N} /+}$, Usp22-/- animals. These parameters were scored to determine the disease activity index (DAI). In both groups, APC wild type and heterozygous, mice with Usp22 loss were characterized by the highest DAI (Figure 18C). Surprisingly, shortly after the DSS treatment $\mathrm{APC}^{1638 \mathrm{~N} /+}$, Usp22 $2^{-/-}$mice died at the age of approximately 12-20 weeks (Figure 18D). Their $\mathrm{APC}^{1638 \mathrm{~N} /+}, \mathrm{Usp}_{22} 2^{+/+}$and Usp22 $2^{+/-}$littermates were characterized by longer survival (Usp22 ${ }^{+/+}$: 25-54 weeks, Usp22 $2^{+/}:$28-39 weeks). In contrast, life expectancy of $\mathrm{APC}^{+/+}$animals was only marginally affected. We found blocked intestines due to intussusceptions or prolapses to be the primary causes of death in $\mathrm{APC}^{1638 \mathrm{~N} /+}$, Usp22 $2^{-/-}$mice. For further analyses the intestinal system was isolated. Notably, the colon length was significantly decreased in APC ${ }^{1638 \mathrm{~N} /+}$, Usp22 $^{-/-}$mice (Figure 18E) indicating strong inflammatory processes (Nordgren et al., 1997). 
A

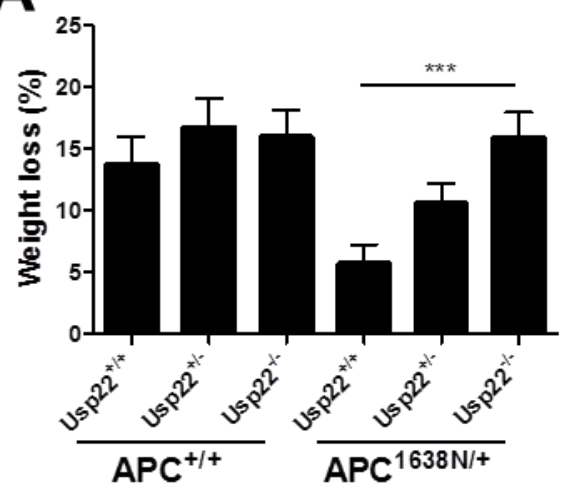

C

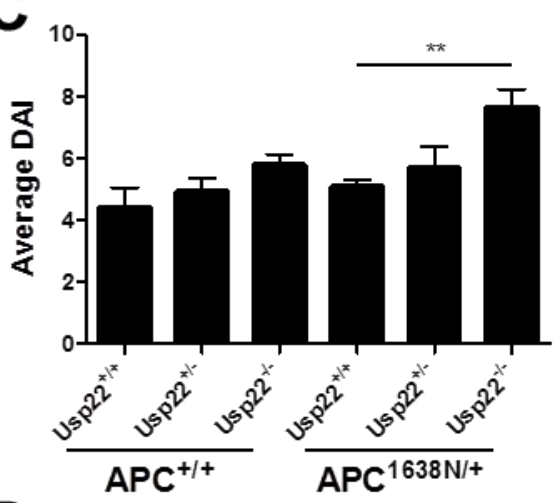

B

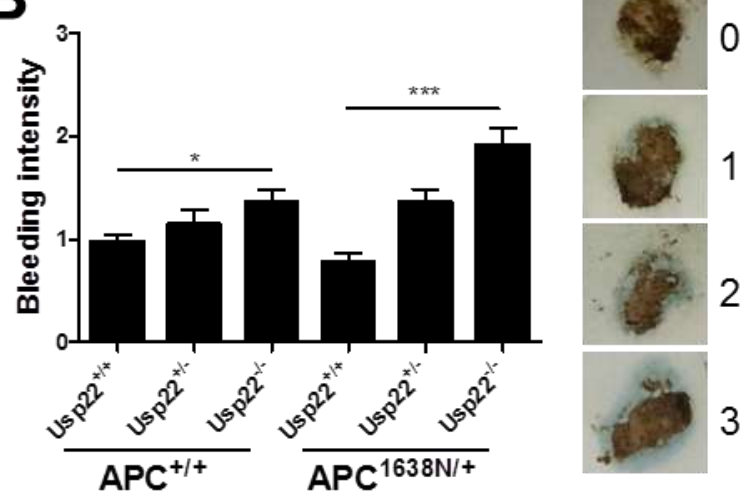

E

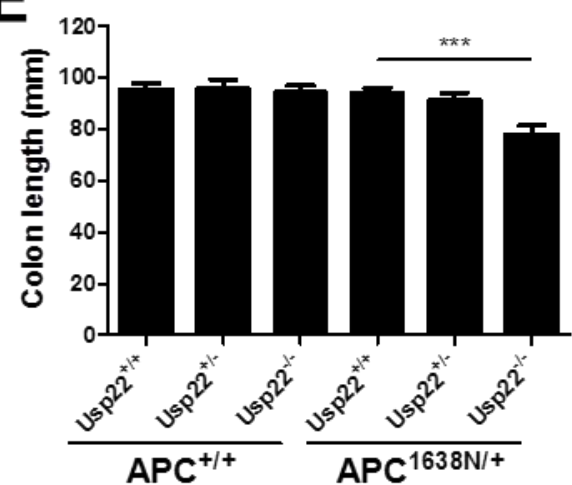

D

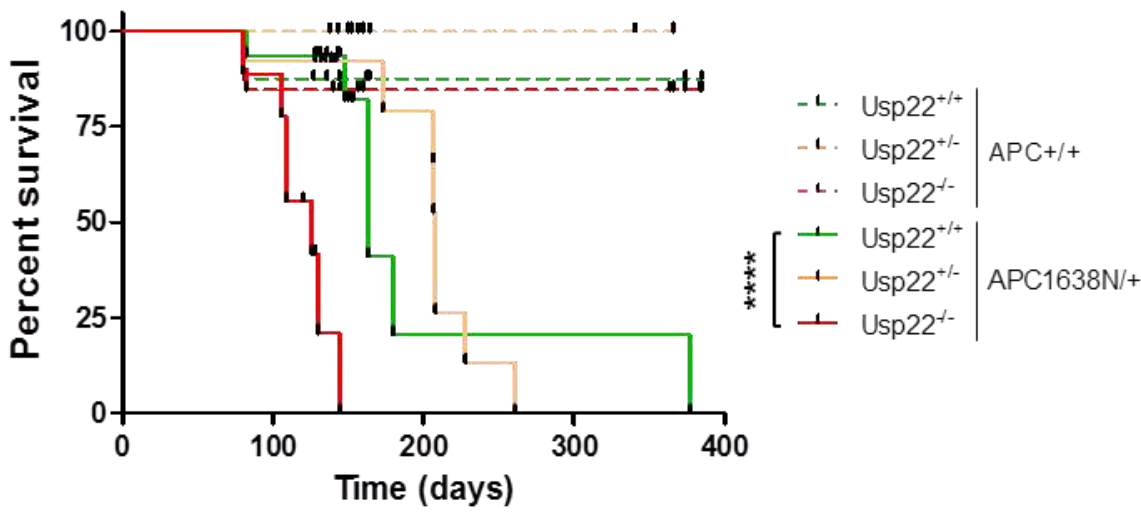

Figure 18: Simultaneous loss of Usp22 and APC mutation lowers life expectancy and increases the DAI in Tam-DSS mice. After starting the DSS treatment health parameters were recorded daily. APC ${ }^{1638 \mathrm{~N} /+}$, Usp22 $2^{-/-}$mice showed $(A)$ increased weight loss, $(B)$ intestinal bleedings and (C) a high average DAI. (D) An APC truncation mutation lead to decreased survival. In comparison to Usp22 wild type and heterozygous animals, upon deletion of Usp22, life expectancy was significantly shortened animals. (E) After sacrifice these mice were characterized by significantly shorter colons. Mean \pm SEM, Student's $t$-test, n-numbers indicated in Table 17.

After sacrificing the mice we checked for metastases and physical abnormalities. While no metastases were detected, increased spleen size was observed in APC ${ }^{1638 \mathrm{~N} /+}$ animals but did 
not correlate with the Usp22 status (data not shown). The intestines were washed and measured. Moreover, tumor locations as well as tumor sizes were recorded. Due to the induction of colitis via DSS treatment, intestinal tumors were found in nearly all animals (Figure 19A, B). In addition, as expected, the truncated APC protein correlated with promoted tumorigenesis. While $\mathrm{APC}^{+/+}$mice had on average 0-7 tumors in the colon and 0-16 in the SI, $\mathrm{APC}^{1638 \mathrm{~N} /+}$ mice showed the presence of approximately $0-33$ tumors in the colon and 2-129 in the SI, respectively. To our surprise, the total number of intestinal tumors was especially elevated upon the loss of Usp22. In $\mathrm{APC}^{+/+}$animals with Usp22 wild type or heterozygous status, the tumor number was slightly lower in the colon compared to Usp22 $2^{-/-}$animals. In the SI the difference among the $\mathrm{APC}^{+/+}$mice was not significant. In contrast, in the $\mathrm{APC}^{1638 \mathrm{~N} /+}$ cohort Usp22 ${ }^{--}$animals developed significantly more tumors in the colon and SI compared to their Usp22 wild type and heterozygous littermates. In fact, tumors were not only more frequent in $\mathrm{APC}^{1638 \mathrm{~N} /+}$, Usp22-/- mice but also characterized by their increased size (Figure 19C, D). The ratio of tumors with a greater surface area than $2 \mathrm{~mm}^{2}$ was significantly increased in APC ${ }^{1638 \mathrm{~N} /+}$, Usp22/- animals. To summarize, in contrast to previously published reports which our hypothesis was based on, we observed that Usp22 loss did not only reduce survival but it also significantly increased tumor burden in inflammation-induced CRC. 

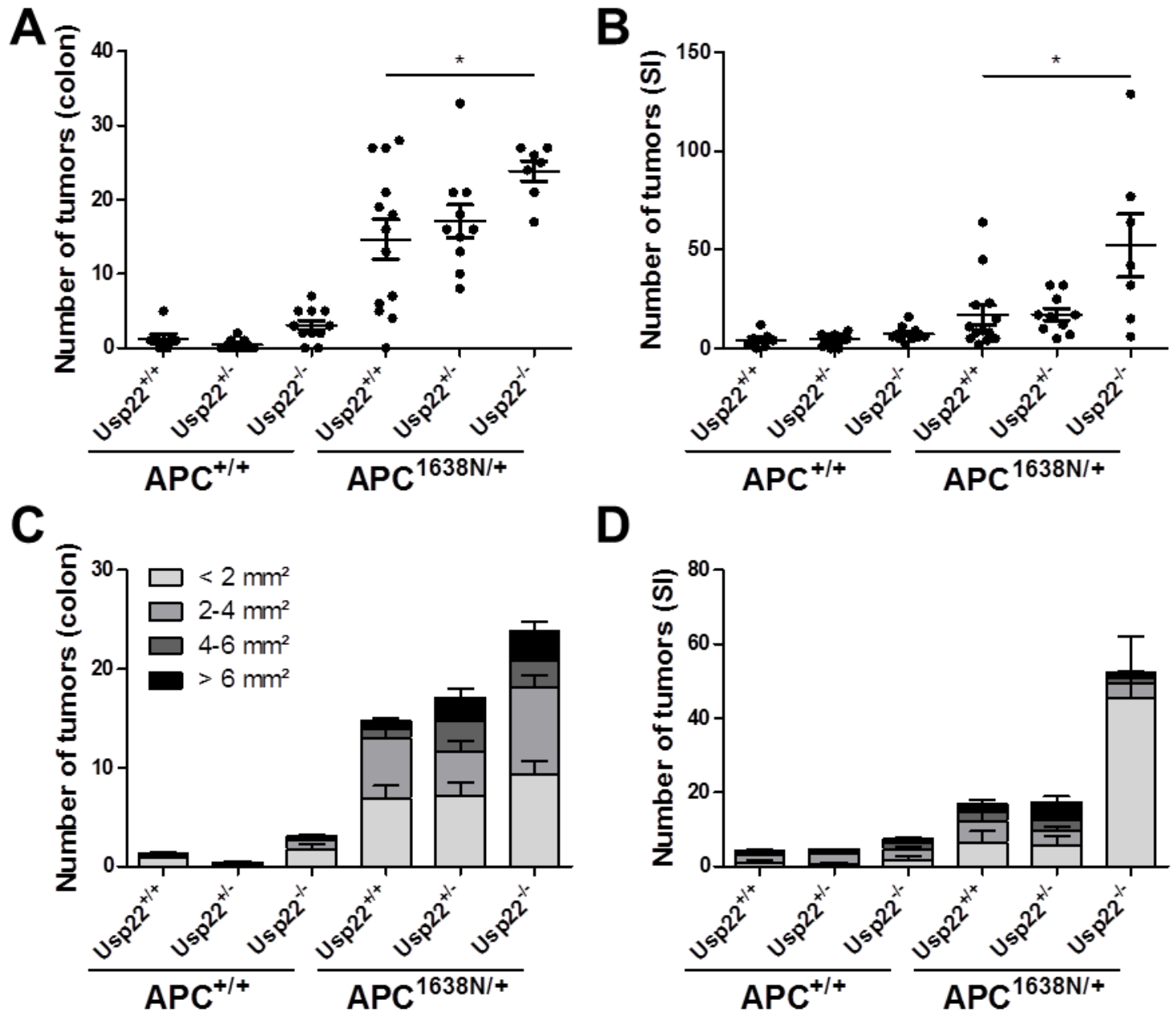

Figure 19: Reduced Usp22 levels significantly elevate intestinal tumor burden. In (A) colon and (B) small intestine loss of Usp22 leads to increased tumor numbers in both, APC ${ }^{+/+}$ and $\mathrm{APC}^{1638 \mathrm{~N} /+}$ mice. (C, D) Apart from tumor frequency, tumor size was elevated in Usp22 ${ }^{-/-}$ mice. Mean \pm SEM, Student's $t$-test, n-numbers indicated in Table 17.

Generally, the intestinal system is divided into several segments with distinct properties and structural features. Moreover, the activity of the Villin promoter decreases from the proximal to the distal intestinal region which can affect the sites of tumor formation as well. Thus, the relative tumor location within the intestinal system was determined. For this purpose, the small intestine and colon were always dissected in the same manner and the tumor distance from the left side and the bottom of the organ was determined. Per genotype the tumor locations of five animals with representative tumor numbers were depicted. As mentioned before, in APC ${ }^{+/+}$ 
mice the tumor number is slightly elevated after the loss of Usp22, however, their distribution is similar to Usp22 $2^{+/+}$littermates (Figure 20). In contrast, in the presence of an APC mutation the deletion of Usp22 leads to an accumulation of tumors in the proximal region of the small intestine.

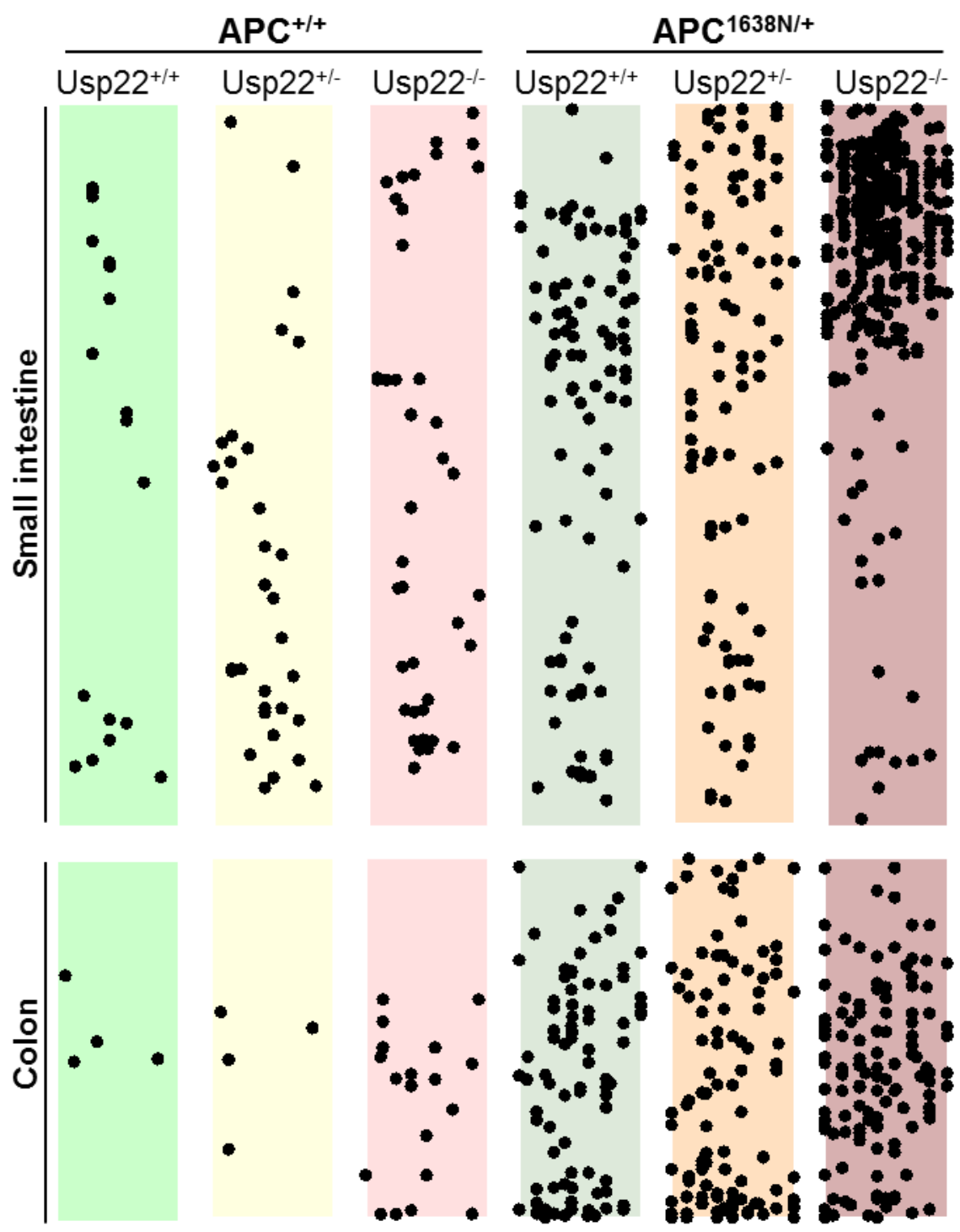

Figure 20: Usp22 loss results in the accumulation of tumors in the proximal SI region. Relative tumor locations from five mice per genotype with representative tumor numbers were depicted. APC ${ }^{1638 \mathrm{~N} /+}$, Usp22 $2^{-/-}$mice were characterized by an accumulation of tumors in the proximal region of the small intestine. 
After measuring tumor size and location, intestines were fixed in formaldehyde and embedded in paraffin. Swiss rolls were prepared to obtain an overview of all regions of the SI and colon. Afterwards, sections were stained with H\&E to visualize organ structures. For morphological studies, we selected the distal third of the small intestine. Generally, the gross morphology of the SI did not vary among genotypes (Figure 21). In $\mathrm{APC}^{+/+}$colons the organ structure was mainly unaffected. Conversely, due to a heterozygous APC mutation, the number of tumors was strongly increased and therefore the organ integrity destroyed.

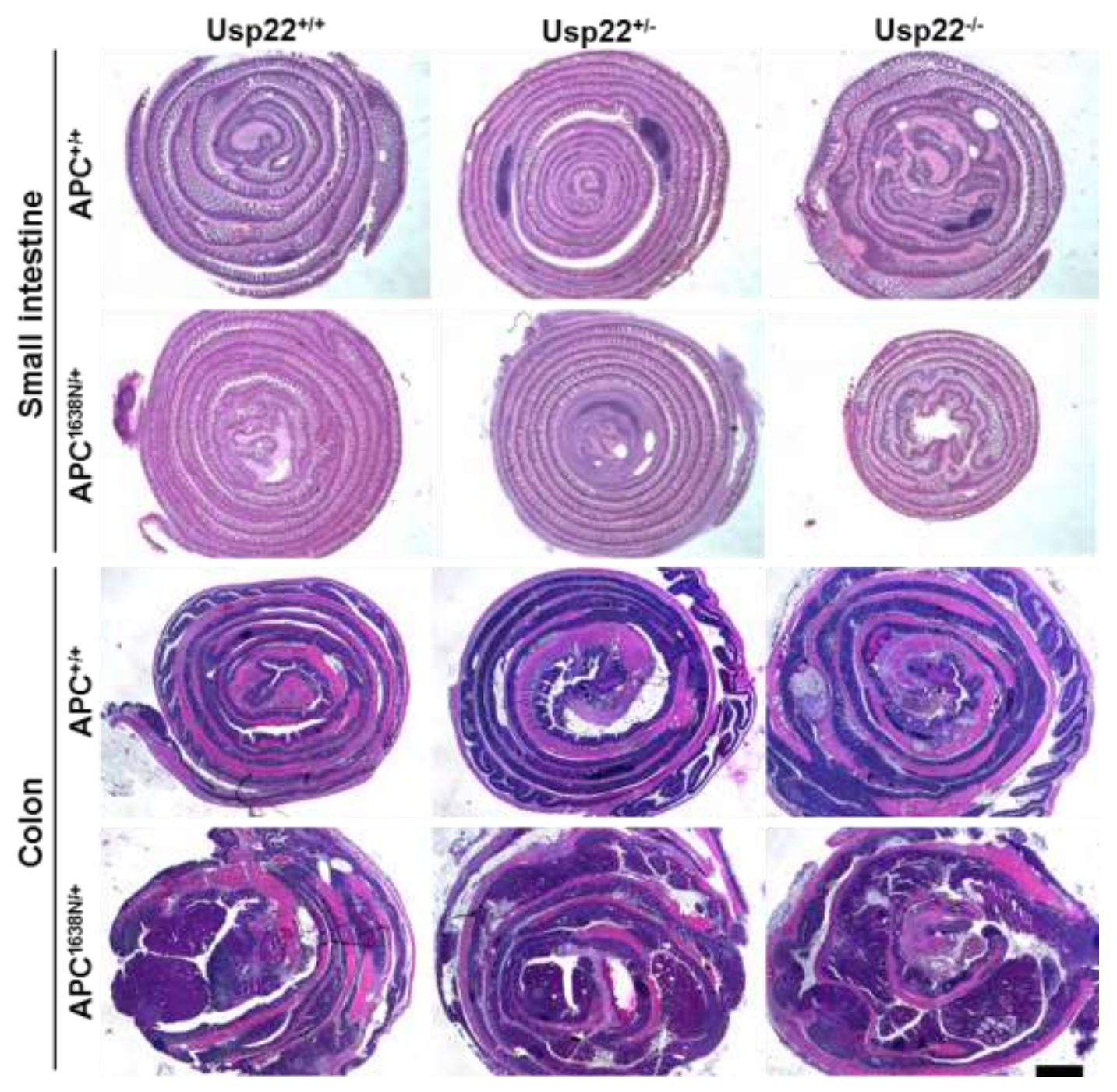

Figure 21: Morphological overview of small intestines and colons. Small intestine and colon sections were stained with $\mathrm{H} \& \mathrm{E}$ and images were prepared to get a morphological overview. Only marginal differences were detected in small intestines among genotypes. In colons of APC ${ }^{1638 \mathrm{~N} /+}$ mice tumors disturb organ integrity. Scale bar: 1,000 $\mu \mathrm{m}$. 


\subsubsection{Usp22 ablation is associated with inflammation and invasive carcinomas}

Based on these H\&E sections we decided to analyze the morphology of the small intestine in more detail. For this purpose we selected healthy SI segments without the presence of MALTs, tumors, etc. (Figure 22A). First, we counted the number of crypts per $100 \mu \mathrm{m}$. In all mice 1-2 crypts per $100 \mu \mathrm{m}$ were detected, however, crypts of Usp22 $2^{-/-}$mice were slightly narrower (Figure 22B). The number of cells per crypt (approximately 20 to 25) did not differ significantly among genotypes (Figure 22C). Similar to the number of crypts, the amount of villi present per $100 \mu \mathrm{m}$ was comparable but slightly decreased in Usp22-/- animals (Figure 22D). In contrast, the villi length, representative for the approximate number of cells per villus, did not vary significantly (Figure 22E). 

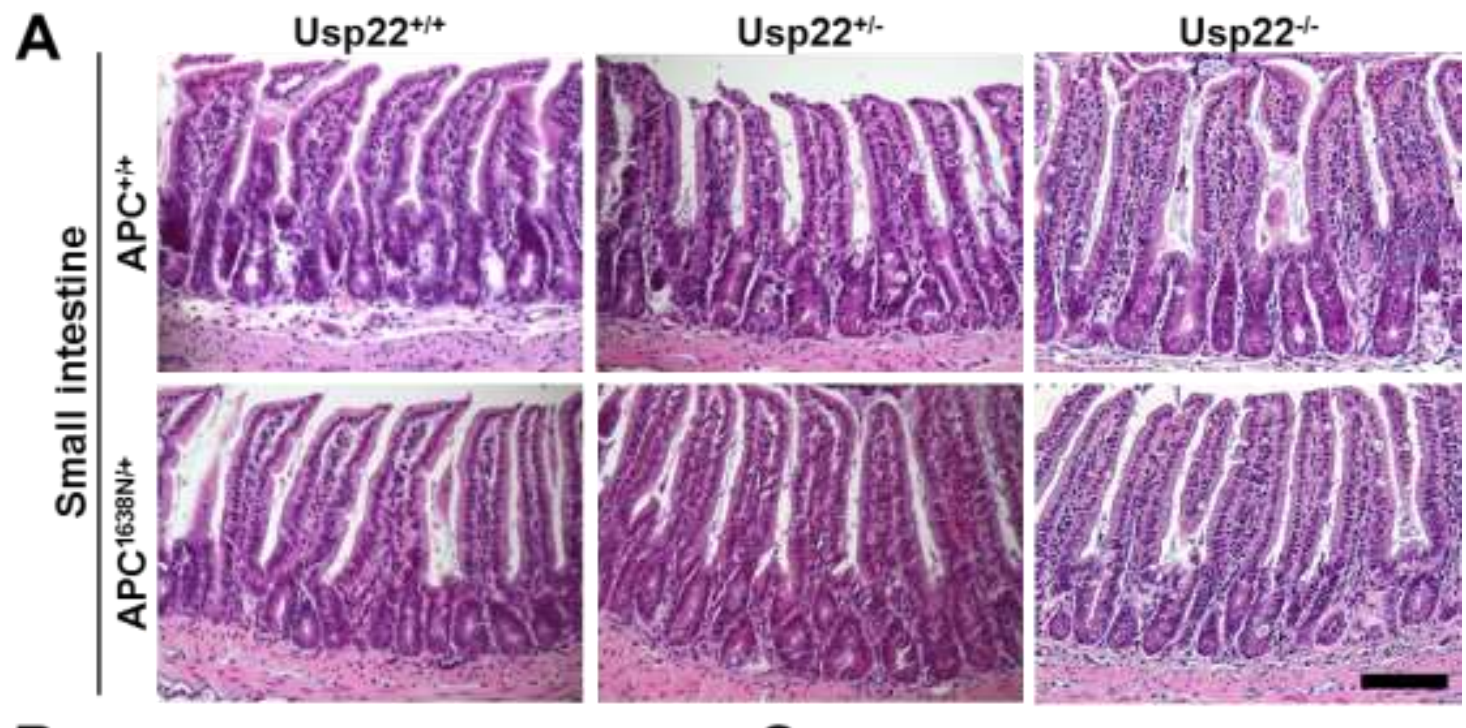

B

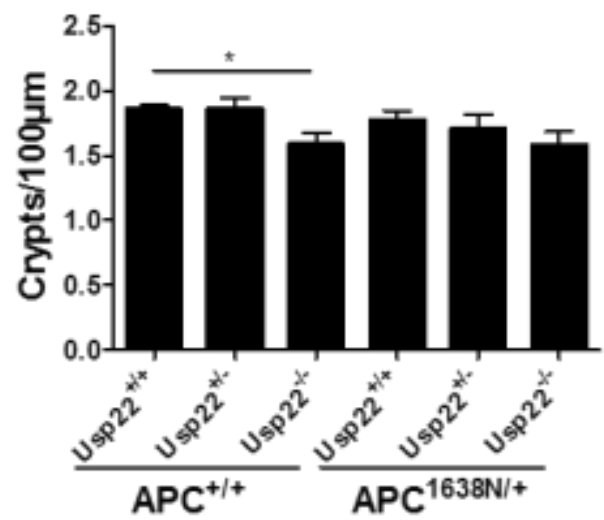

C

D

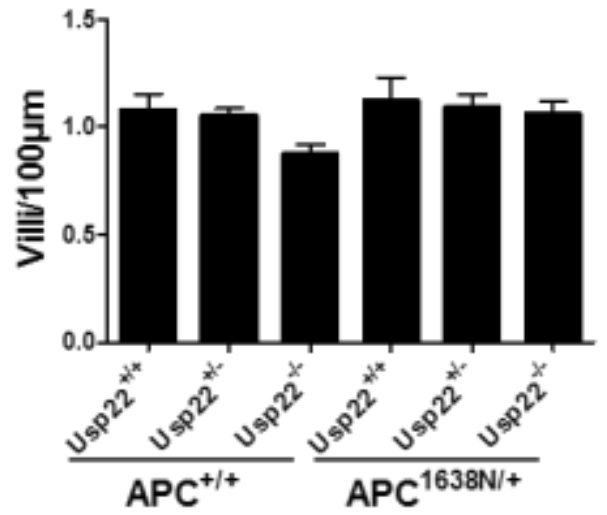

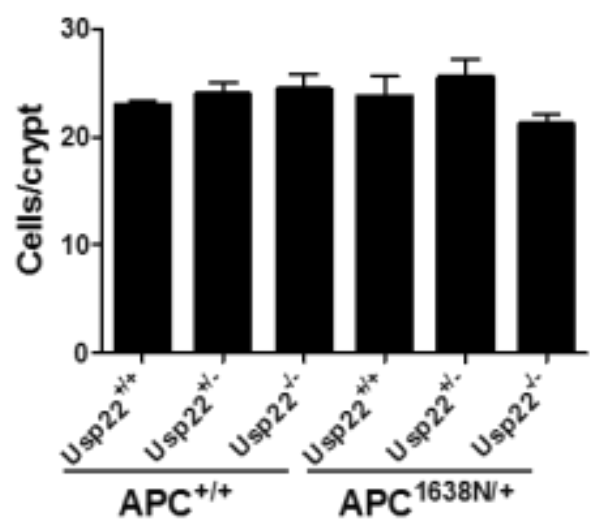

E

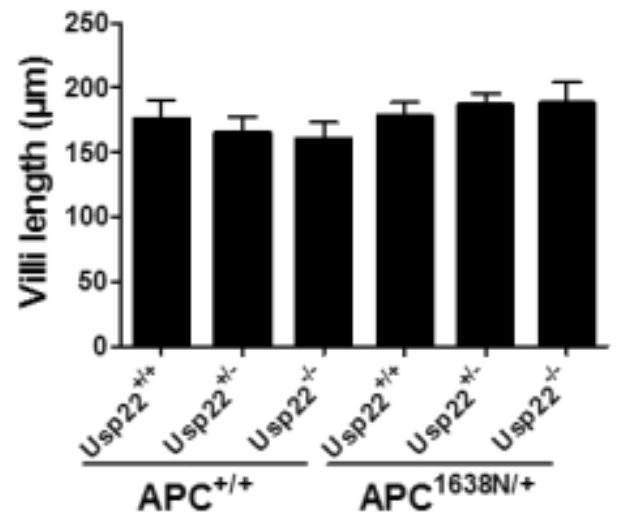

Figure 22: Conditional intestinal loss of Usp22 does not affect small intestine morphology. (A) To evaluate small intestine morphology more in detail, H\&E-stained sections were assessed. Scale bar: $100 \mu \mathrm{m}$. (B) There was no difference among genotypes when comparing the number of crypts per $100 \mu \mathrm{m}$ and (C) cells per crypt. (D) The number of villi per $100 \mu \mathrm{m}$ as well as the (E) villi length was not changed. Mean \pm SEM, Student's $t$-test, $n$ numbers indicated in Table 17. 
In addition, we observed the morphology of the colon in these mice (Figure 23A). It must be mentioned that the rate of intact colon segments was reduced in $\mathrm{APC}^{1638 \mathrm{~N} /+}$ mice due to the high tumor frequency. However, at least 50 crypts from different organ regions were evaluated. On average two crypts were detected per $100 \mu \mathrm{m}$ in all mice (Figure 23B). Moreover, the crypts were characterized by a depth of approximately 150 to $200 \mu \mathrm{m}$ (Figure 23C). Taken together, the morphology and integrity of the small intestine as well as the colon was only marginally affected by the loss of Usp22.

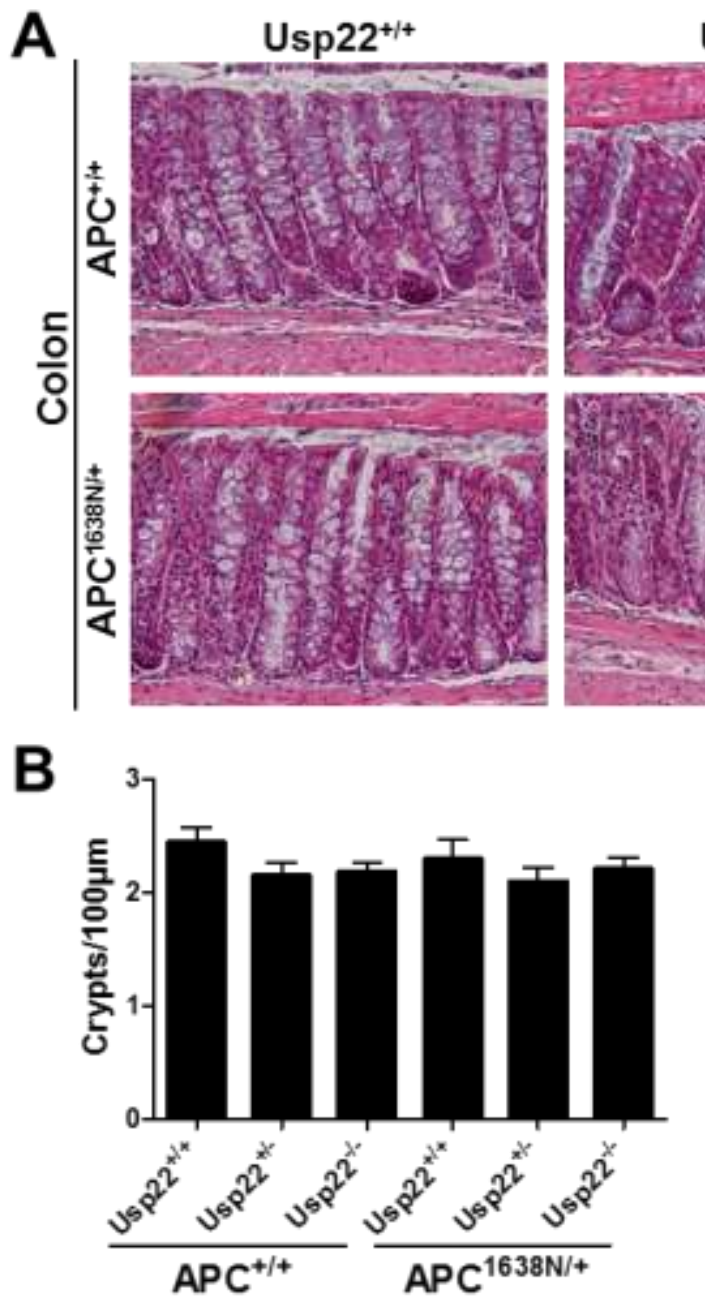

Usp22 $2^{+/-}$

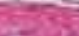
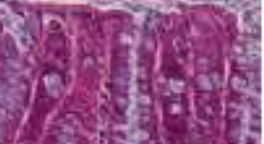

2.0.
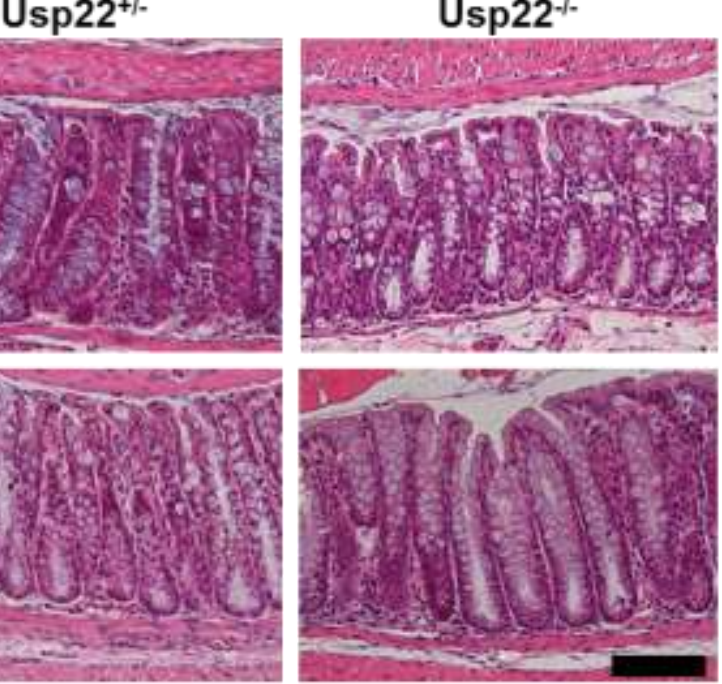

C

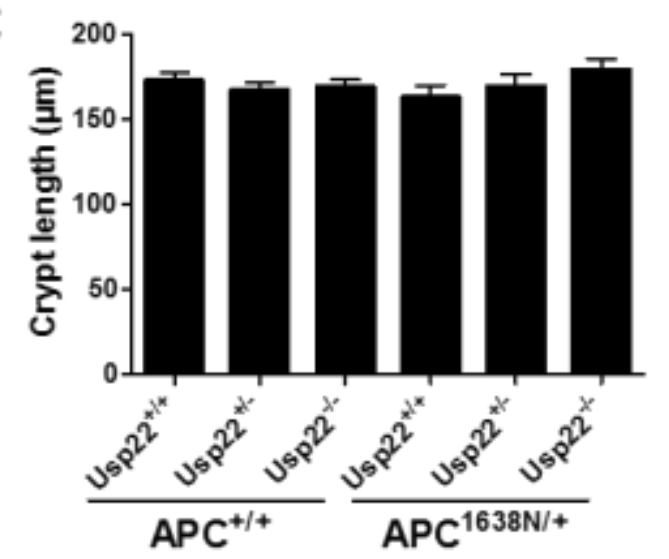

Figure 23: The morphology of healthy colon segments is not affected by reduced Usp22 expression. (A) Colon morphology was evaluated on H\&E-stained sections in intact organ regions. Scale bar: $100 \mu \mathrm{m}$. (B) In intact colon segments the number of crypts per $100 \mu \mathrm{m}$ and (C) crypt length did not differ among genotypes. Mean \pm SEM, Student's $t$-test, n-numbers indicated in Table 17. 
Besides determining the tumor number/location and the morphology of healthy intestinal structures, tumors were also evaluated based on their aggressiveness. Generally, during progression tumors can accumulate mutations influencing their growth characteristics (Fearon and Vogelstein, 1990). Once a tumor spreads beyond the inner muscular lining of the colon, the muscularis mucosae, it becomes invasive. We assessed H\&E-stained colon sections for this phenomenon and could indeed detect several occurrences (Figure 24A).

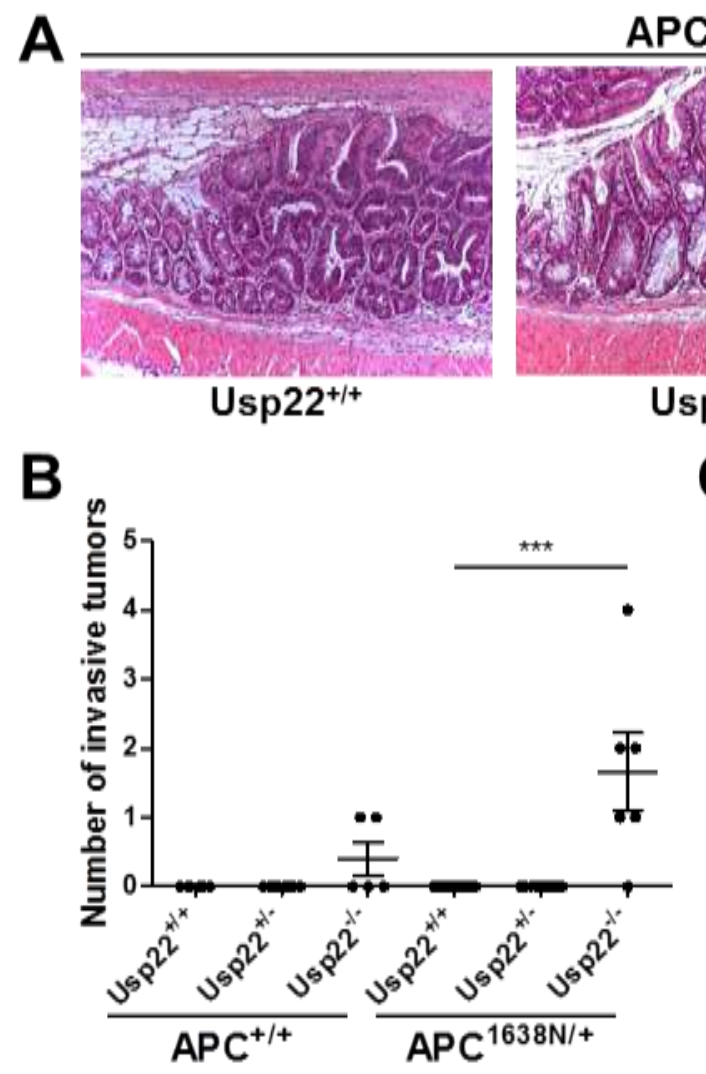

$\operatorname{APC}^{1638 N /+}$
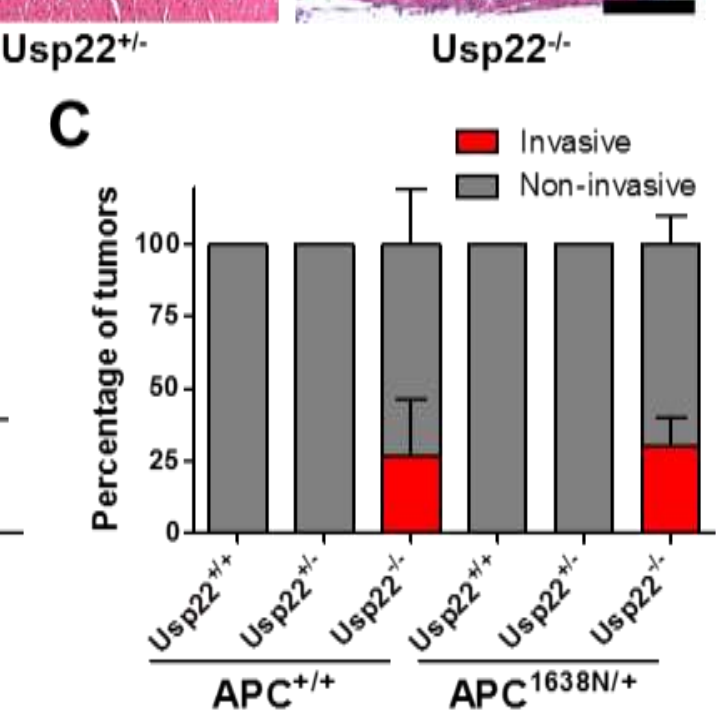

Figure 24: Invasive carcinomas are exclusively present in Usp22-- mice. (A) H\&E-stained colon sections were assessed for the presence of invasive carcinomas which migrate though the muscularis mucosae as shown for Usp22-- mice. (B) Invasive carcinomas were only found in Usp22 $2^{--}$animals, in both $\mathrm{APC}^{+/+}$and $\mathrm{APC}^{1638 \mathrm{~N} /+}$ background. (C) Compared to the total tumor number in the colon the average abundance of invasive carcinomas was approximately $20 \%$ higher in Usp22 $2^{-/-}$mice. Mean \pm SEM, Student's $t$-test, n-numbers indicated in Table 17.

Interestingly, invasive tumors were exclusively found in Usp22 ${ }^{-/-}$animals (Figure 24B), independent of the APC status. In total, the number of invasive tumors was higher in the APC 
mutated mice, however, when taking the total tumor number into account, the percentage of invasive tumors was similar in Usp22 $2^{-/-}$animals with $\mathrm{APC}^{+/+}$and $\mathrm{APC}^{1638 \mathrm{~N} /+}$ status (Figure 24C). This finding clearly demonstrates that not only the tumor number is elevated after Usp22 loss but also their tumor aggressiveness is higher leading to a higher probability of metastasis.

While analyzing H\&E-stained colon sections we detected mucus accumulations in several animals. Generally, these mucus cysts were present below the lamina muscularis mucosae as encapsulated structures within the muscle layer (Figure 25A). Even extremely large cysts were always surrounded by muscle tissue (black arrowhead). Interestingly, besides these mucus cysts, in Usp22 $2^{-/-}$mice carcinomas with a mucinous appearance were detected sporadically. In these lesions not only encapsulated cysts (yellow arrowhead) as shown in the first two images were present but also mucus accumulations surrounded by epithelial cells (red arrowhead). Notably, these mucus accumulations and tumors with a mucinous appearance were predominantly detected in Usp22 knockout animals (Figure 25B). Moreover, we noticed that Usp22 $^{-/-}$animals were characterized by a high frequency of MALTs (Figure 25C). As a crucial component of the mucosal immunity, mucosa-associated lymphoid tissues are routinely found in the intestine; however, their high abundance could result from an increased antiinflammatory response due to colitis. Colon sections were evaluated and an increased MALT frequency was detected in Usp22-/- mice while it could only be found sporadically in Usp22 wild type and heterozygous animals. This finding was independent from the APC status. These results reveal increased lymphocyte accumulation rates after the loss of Usp22 and interestingly, also a mucinous phenotype. 

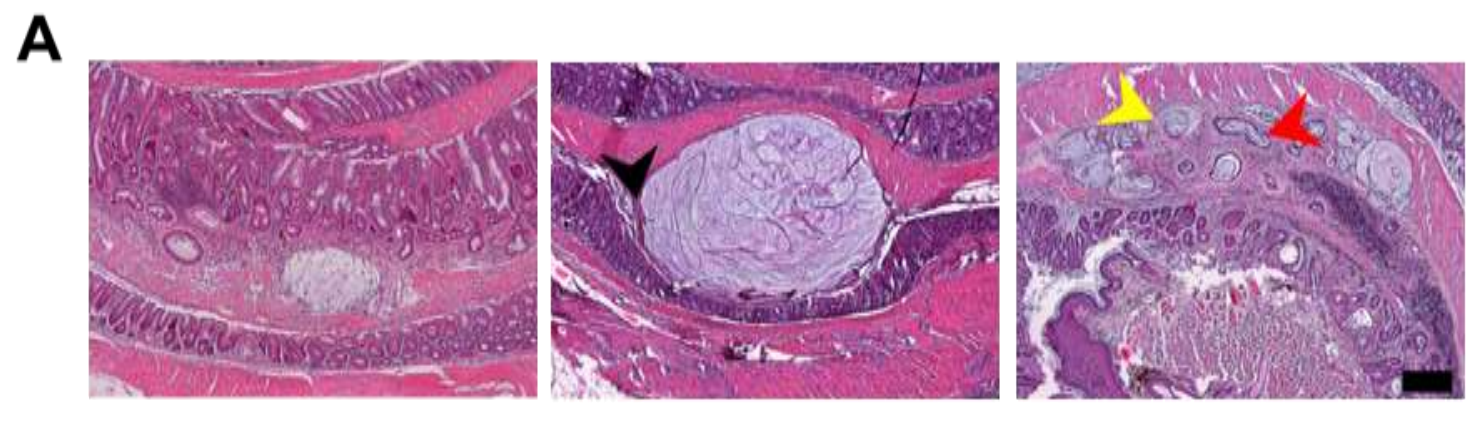

B

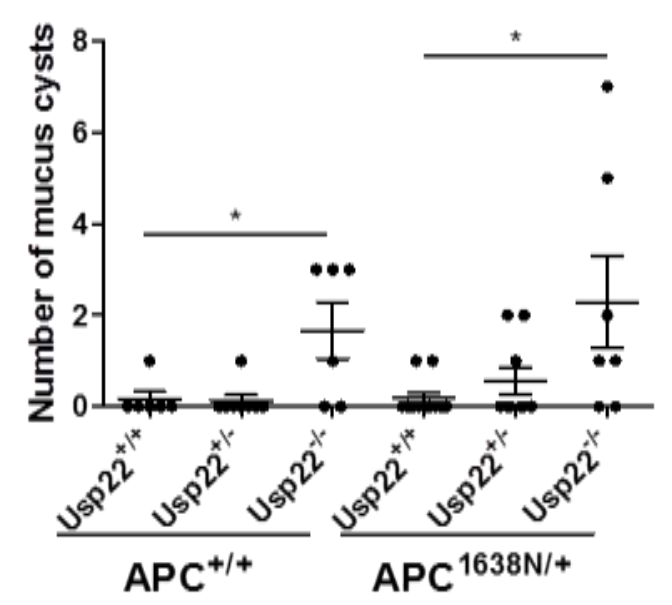

C

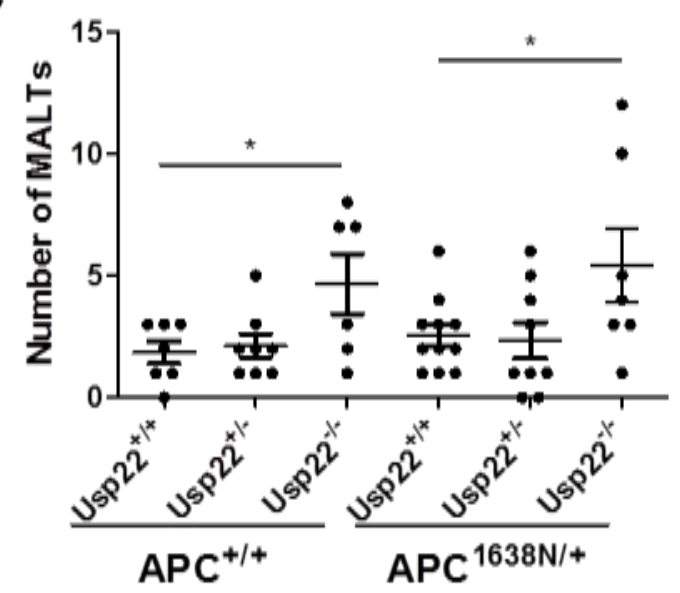

Figure 25: Mucinous presentation and elevated frequency of MALTs in Usp22-- colons. (A) Mucus cysts of different sizes were found in several animals and were frequently surrounded by muscle tissue (black arrow). In Usp22-- colons carcinomas with a mucinous appearance were present which contain cysts surrounded by muscle tissue (yellow arrow) or by epithelial cells (red arrow). Scale bar: $200 \mu \mathrm{m}$. (B) These mucus cysts were predominantly found in Usp22 $2^{--}$mice. (C) To assess the presence of immune defense-associated lymphoid aggregates, MALTs have been counted in H\&E-stained colon sections. An elevated abundance of MALTs was detected after the loss of Usp22. Mean \pm SEM, Student's $t$-test, nnumbers indicated in Table 17.

Based on these findings, the inflammatory response upon DSS treatment was investigated in more detail. A well-accepted method to evaluate the inflammation based on H\&E staining is the determination of the Histo-score (H-score). The scoring system is based on the intactness of the colonic epithelium and lymphocyte infiltration. It ranges from 0 to 3 (Figure 26A) considering normal epithelium (score 0), mild inflammation in which lymphocyte infiltration pushed crypts apart (1), lymphocytes accumulations interfering with crypt structure (2) and crypt integrity which is destroyed by lymphocytes and where the epithelial lining (black 
A 1
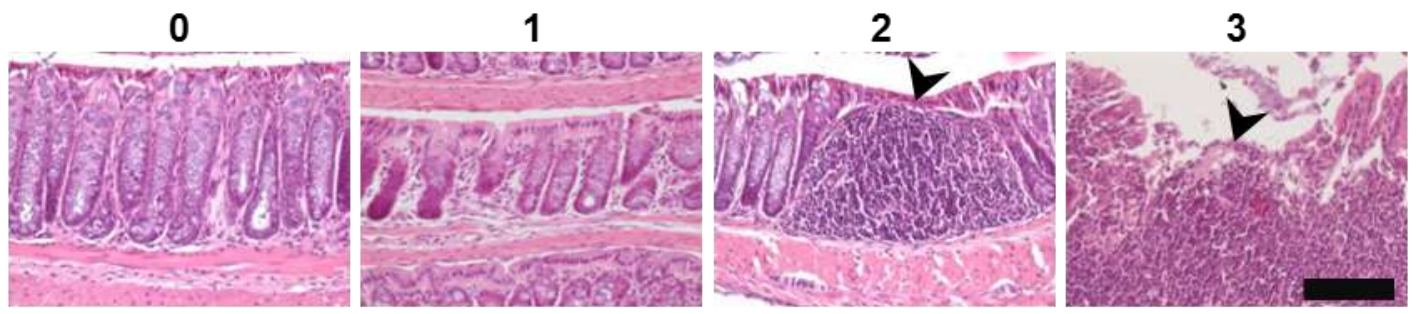

B
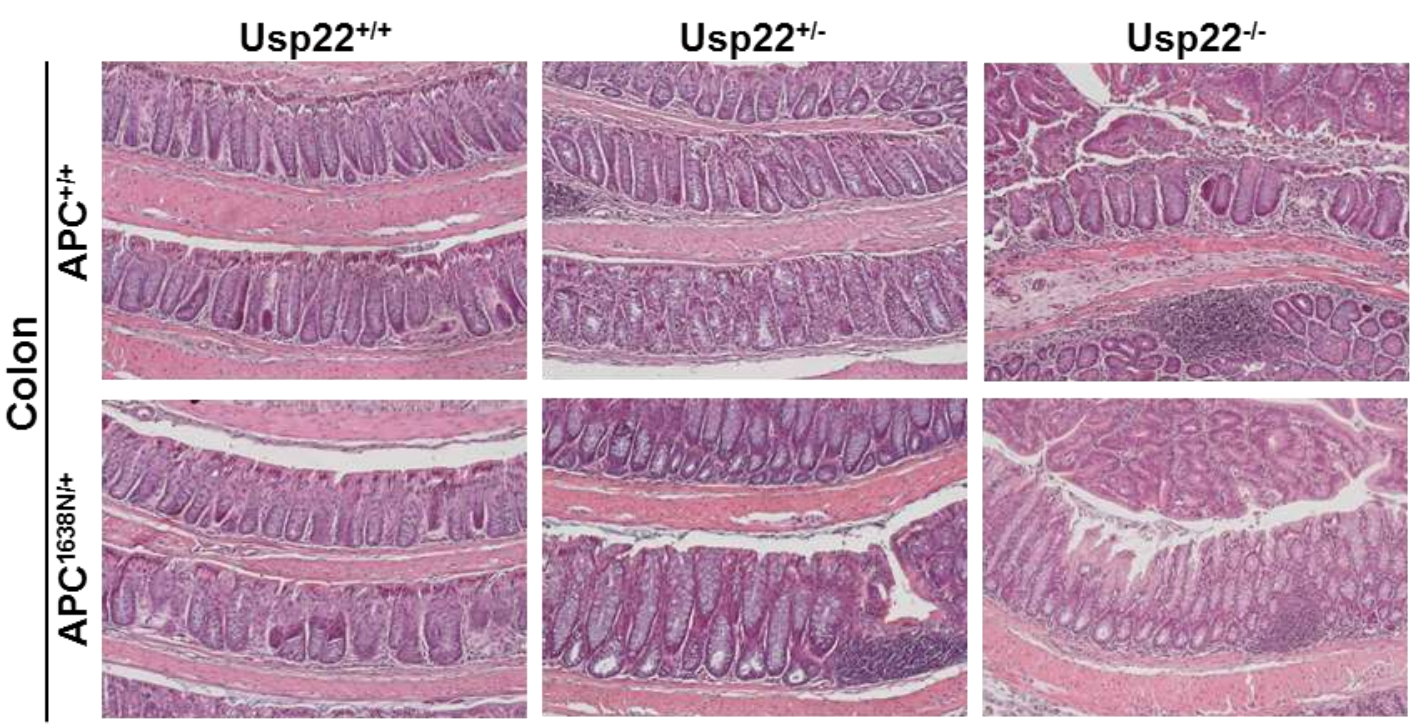

C

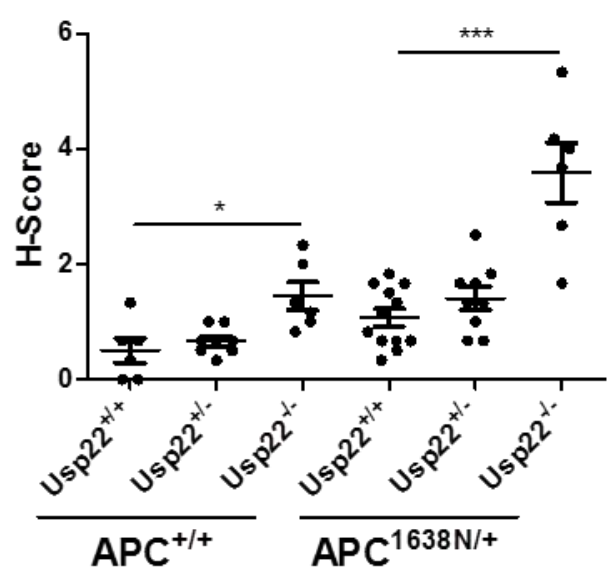

D

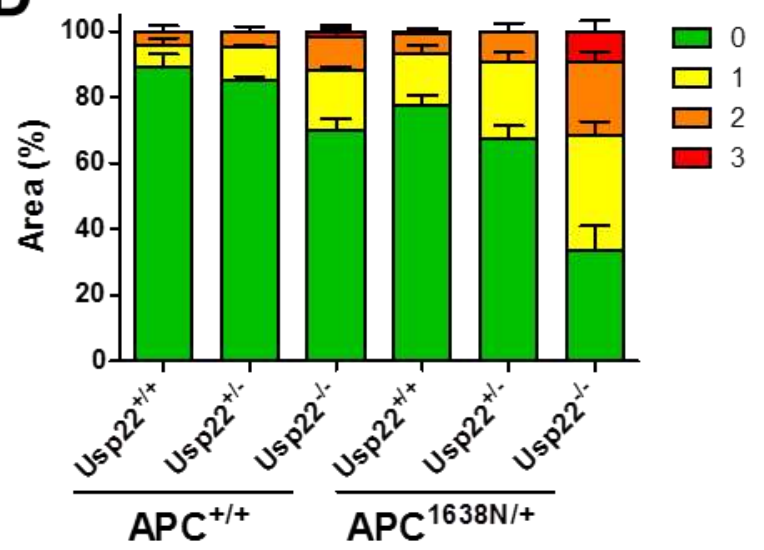

Figure 26: High occurrence of inflammation-associated epithelial damage in Usp22-animals. (A) Inflammation-associated alterations of the epithelium were assessed on H\&Estained colon sections. Analyses were based on the $\mathrm{H}$-score ranging from 0 to 3 reflecting increasing degrees of epithelial damage. During severe inflammation the epithelial lining (black arrowhead) is destroyed. Scale bar: $100 \mu \mathrm{m}$. (B) The loss of Usp22 was associated with severely inflamed intestinal epithelium. Scale bar: $200 \mu \mathrm{m}$. (C) Usp22 $2^{-/}$animals were characterized by higher $\mathrm{H}$-scores (D) caused by many segments with mild (1), medium (2) or severe (3) damage. Mean \pm SEM, Student's $t$-test, $n$-numbers indicated in Table 17. 
arrowhead) on top is absent (3). We calculated the H-score and found inflammation in the majority of mice due to the DSS treatment. In both groups, $\mathrm{APC}^{+/+}$and $\mathrm{APC}^{1638 \mathrm{~N} /+}$, there were only marginal differences between Usp22 wild type and heterozygous animals. Notably, in both these groups, Usp22-/- mice were characterized by a high proportion of epithelium showing medium to severe signs of inflammation (Figure 26B). Accordingly, we detected significantly elevated $\mathrm{H}$-scores (Figure 26C) due to an increased rate of severely damaged tissue (Figure 26D). In summary, even several weeks after ending the DSS treatment colons of Usp22 knockout mice were partially inflamed in contrast to the Usp22 wildtype and heterozygous animals which showed overall less inflammation.

Finally, we aimed to evaluate H2Bub1 levels in healthy colon epithelium and tumors. For this purpose, Tam-DSS colon sections were stained for H2Bub1 by immunohistochemistry. As expected, the finding was similar to our observations made in Usp22lacZ small intestine sections. We did not see any differences in H2Bub1 levels in normal colon crypts (Figure 27A). In contrast, H2Bub1 levels in colorectal tumors were highly heterogeneous. While cells were positively stained in some tumor regions, especially poorly differentiated areas were characterized by the absence of H2Bub1 (Figure 27B). Thus, our previous findings obtained from this cohort seem to be independent of H2Bub1 levels.

To sum up, in the first cohort of experimental mice (Tam-DSS) we assessed the effect of Usp22 on colitis and subsequent formation of intestinal tumors. Interestingly, shortly after finishing the DSS treatment, APC ${ }^{1638 \mathrm{~N} /+}$, Usp22-/- animals died. To our surprise, wild type Usp22 expression attenuated tumor growth while tumor burden and the presence of invasive tumors were exacerbated by Usp22 knockout. Notably, in Usp22 $2^{-/-}$mice severe inflammation was detected even several weeks after DSS treatment and the colon as well as some tumors were characterized by a mucinous phenotype. 


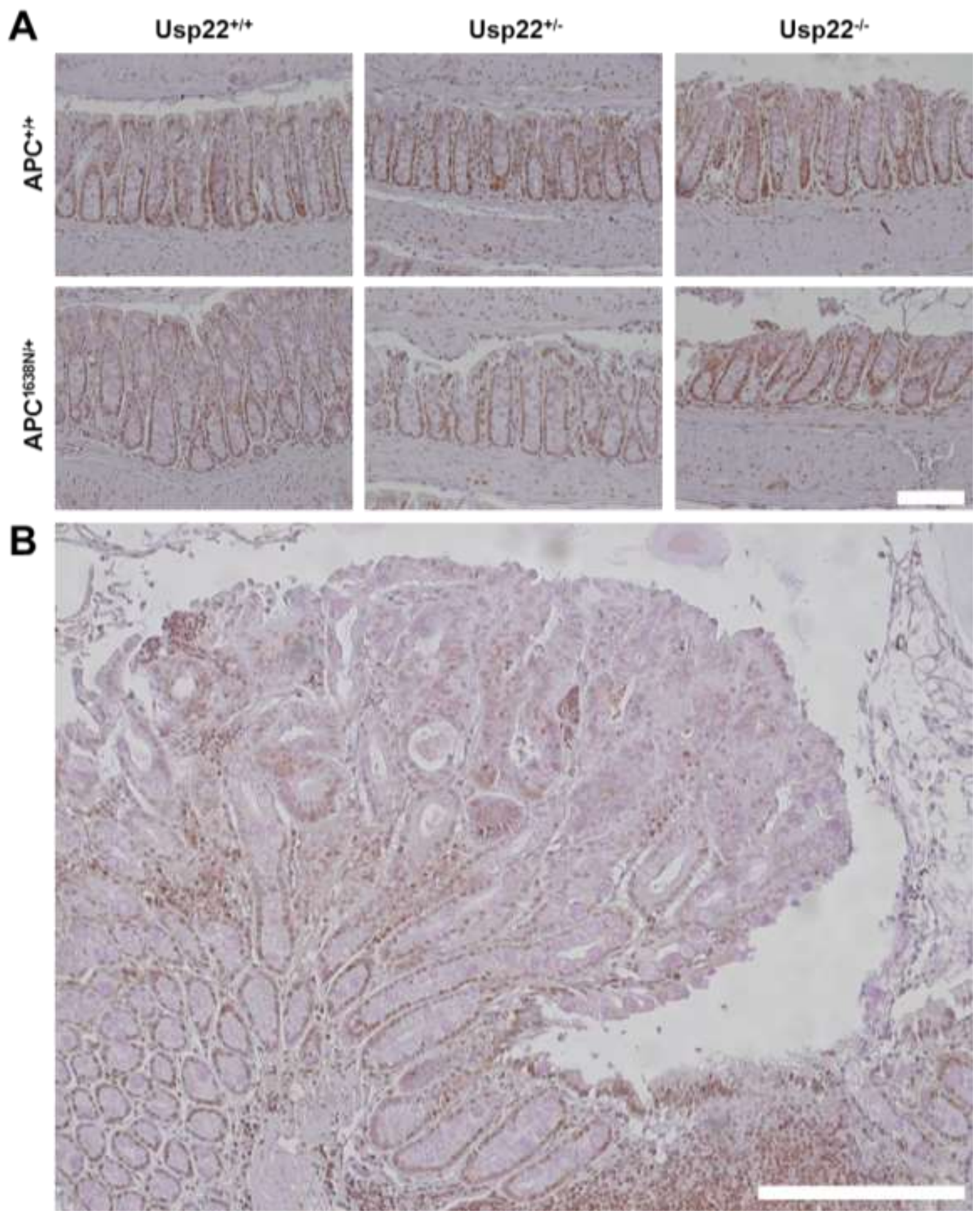

Figure 27: H2Bub1 levels are independent of the Usp22 status but show intratumoral variations. (A) Immunohistochemistry was performed for $\mathrm{H} 2 \mathrm{Bub1}$ on healthy colon segments and the number of positive cells, their localization and staining intensity was independent of the Usp22 status. Scale bar: $100 \mu \mathrm{m}$. (B) The intratumoral H2Bub1 distribution was highly diverse. While in the healthy epithelium H2Bub1 was detected, its levels decreased in poorly differentiated tumor regions. Representative photo of an $\mathrm{APC}^{1638 \mathrm{~N} /+}$; Usp22 $2^{-/-}$colon tumor. Scale bar: $200 \mu \mathrm{m}$. 


\subsubsection{Inflammation-induced CRC: Colitis prior to intestinal Usp22 deletion (DSS-Tam)}

\subsubsection{Inducing colitis prior to Usp22 loss reduces tumor and inflammation burden}

In the first mouse cohort (Tam-DSS) we induced the Usp22 knockout prior to DSS treatment to observe how inflammation and subsequent tumor formation were affected by the absence of Usp22. In a second cohort for inflammation-associated CRC (treatment group 2: DSS-Tam), colitis was induced at an age of 10 weeks followed by an Usp22 knockout after a short recovery period. In this group we avoided the possibility that mice react differently to DSS treatment due to their genetic background which in turn could have affected tumorigenesis. Health-related parameters were recorded weekly at the onset of DSS administration until three weeks after Tamoxifen injection. Surprisingly, body weight was only marginally affected by induction of colitis (Figure 28A) while mild intestinal bleedings were observed in all animals (Figure 28B). Diarrhea was rarely observed (data not shown) and the disease activity index was comparable among all genotypes (Figure 28C). Similar to our previous findings, APC ${ }^{1638 \mathrm{~N} /+}$, Usp22-/- mice were characterized by decreased survival rates (Figure 28D). However, compared to $\mathrm{APC}^{1638 \mathrm{~N} /+}$ mice with Usp22 wild type or heterozygous status, this finding was not statistically significant. After sacrificing the experimental animals, we could not detect any differences in the colon length (Figure 28E). 

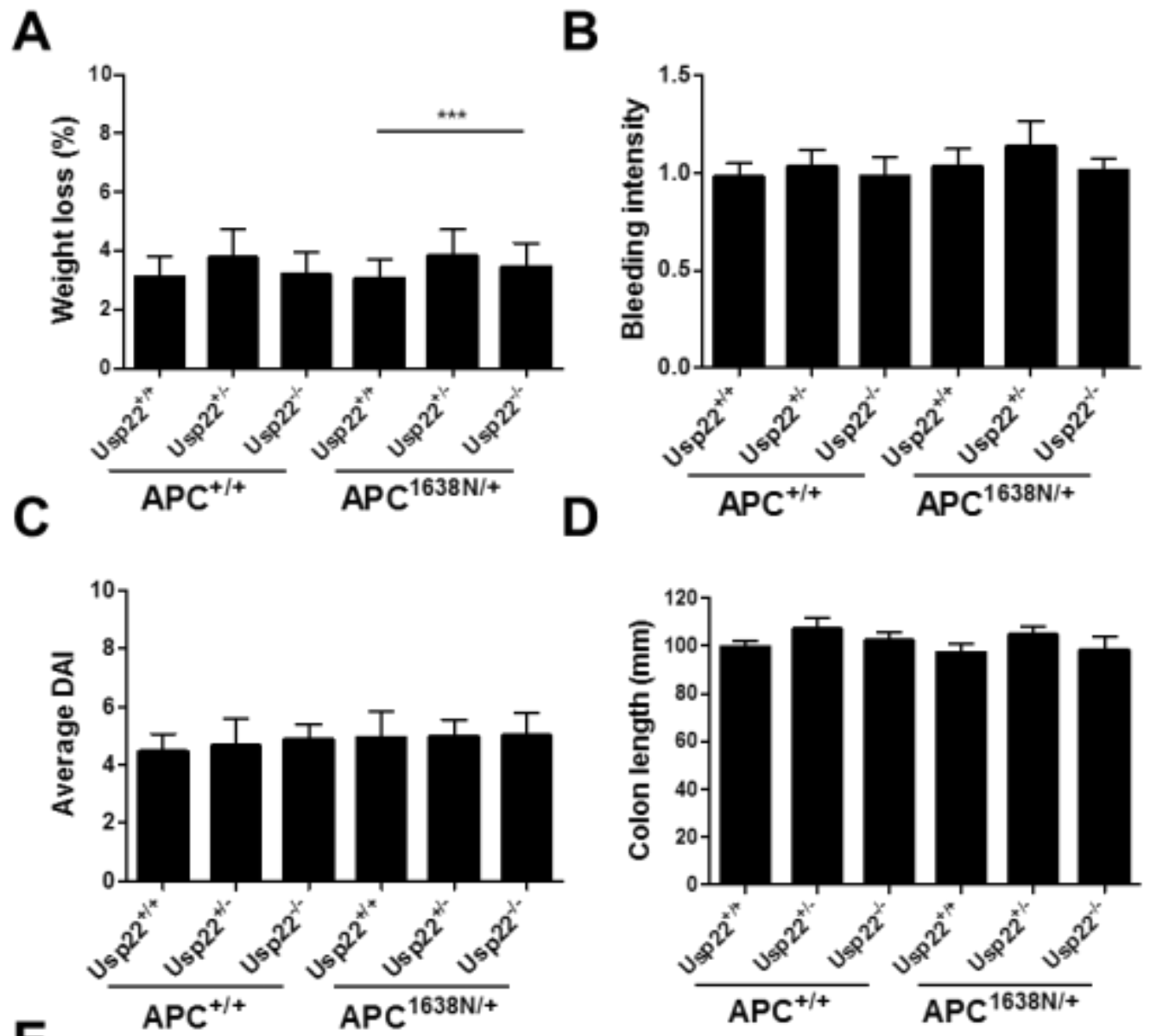

E

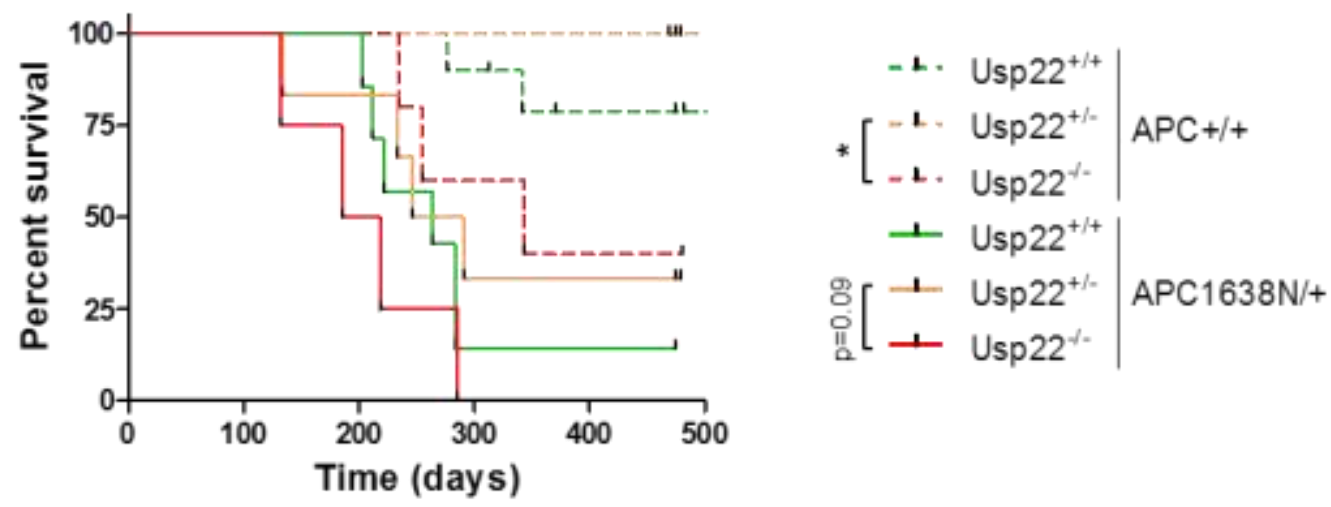

Figure 28: Usp22 loss after DSS treatment does not affect the DAl but decreases survival rates. (A) Body weight and (B) bleeding intensity were recorded regularly and did not differ among genotypes. (C) The average DAl was similar for all animals. (D) Survival rates were decreased for Usp22-- mice independent of the APC status. (E) Colon length was similar in all mice. Mean \pm SEM, Student's $t$-test, n-numbers indicated in Table 17. 
Similar to our aforementioned approach in Tam-DSS mice, we counted and measured all intestinal cancer lesions. Consistent with our previous observations, we could detect elevated tumor burden in Usp22-- animals (Figure 29A, B). While the effect was only minimal in the $\mathrm{APC}^{+/+}$cohort, the tumor burden was obviously increased in $\mathrm{APC}^{1638 \mathrm{~N} /+}$ mice with an Usp22 loss. Furthermore, tumors found in $\mathrm{APC}^{1638 \mathrm{~N} /+}$, Usp22 ${ }^{-/-}$mice were bigger than the cancer lesions present in their littermates (Figure 29C, D).
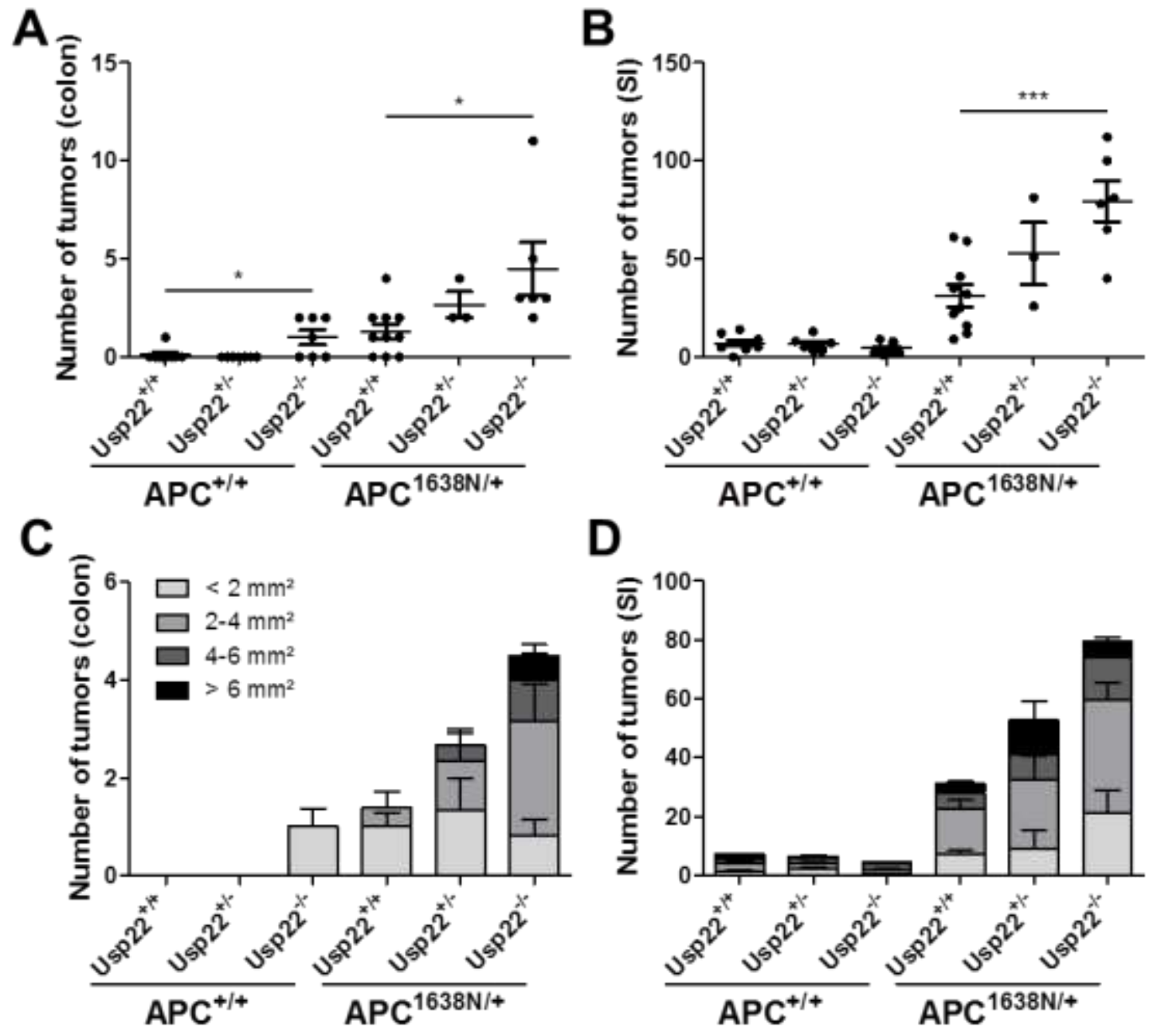

Figure 29: Elevated tumor frequency and size in DSS-Tam APC ${ }^{1638 \mathrm{~N} /+}$, Usp22-- mice. Tumors were counted in the (A) colon and (B) small intestine. There were only marginal effects among $\mathrm{APC}^{+/+}$mice while the frequency of cancer lesions was increased in $\mathrm{APC}^{1638 \mathrm{~N} /+}$, Usp22/- mice compared to the other APC ${ }^{1638 \mathrm{~N} /+}$ animals. (C, D) The loss of Usp22 in an APC ${ }^{1638 \mathrm{~N} /+}$ background resulted in the formation of intestinal tumors with enlarged sizes. Mean $\pm S E M$, Student's $t$-test, n-numbers indicated in Table 17. 
Finally, we compared the tumor location within the intestinal system. In the Tam-DSS mouse cohorts we could observe an accumulation of cancer lesions in the proximal and medial segment of the small intestine. In this cohort, even though the total tumor number was lower, a similar tendency was demonstrated (Figure 30).

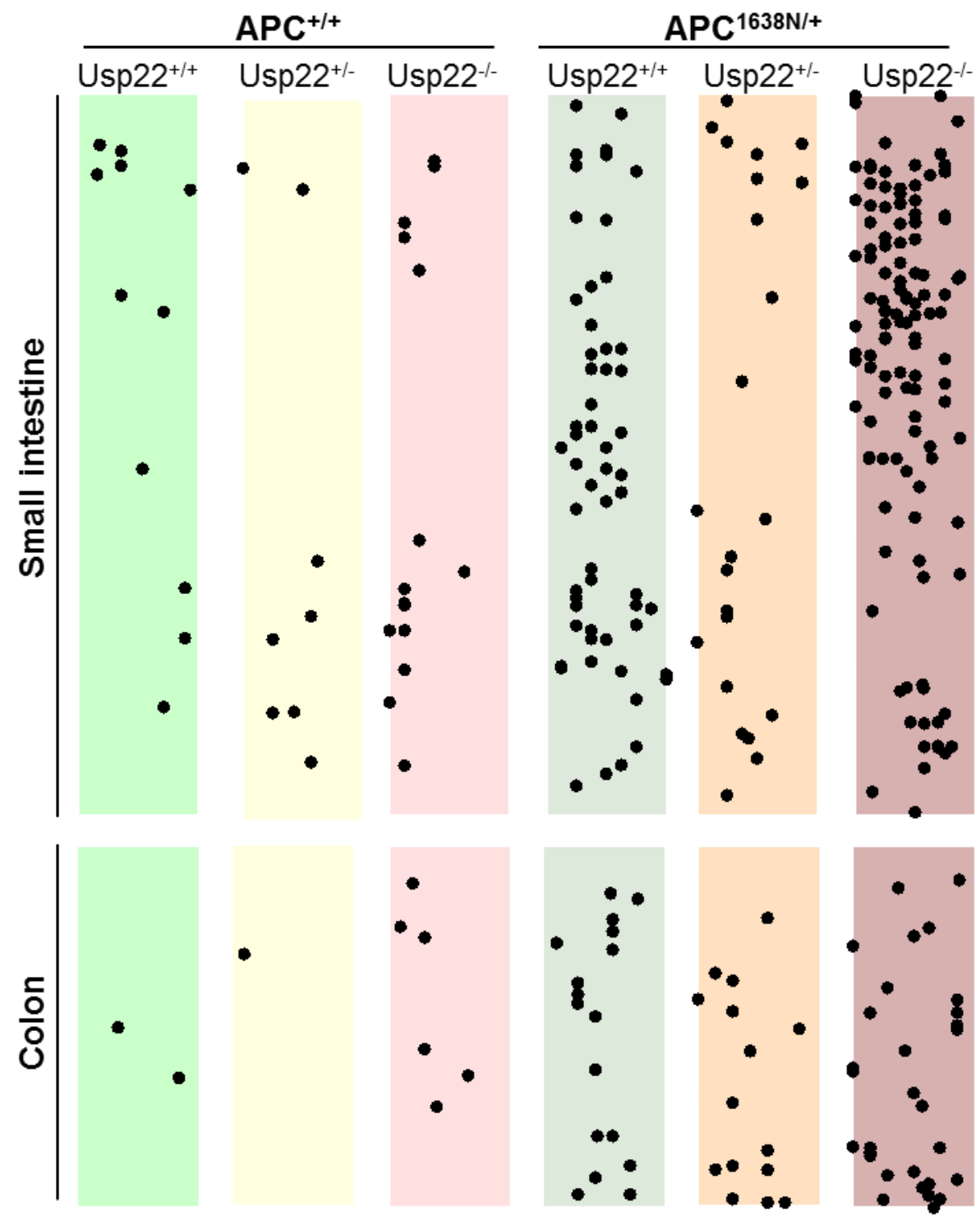

Figure 30: Tumors tend to accumulate in the proximal/medial SI segment of DSS-Tam animals. Relative tumor locations from five mice per genotype with representative tumor burden were visualized. There is a tendency that tumors accumulate in the proximal/medial region of the small intestine. 
Furthermore, we assessed whether the inflammation burden of the DSS-Tam cohort is comparable to the Tam-DSS group. Colon sections were stained with H\&E and a slight increase in lymphocyte infiltration was observed (Figure 31A). The corresponding $\mathrm{H}$-scores were found to be increased in animals with an intestinal Usp22 deletion (Figure 31B) because of more damaged epithelial regions (Figure 31C).

A
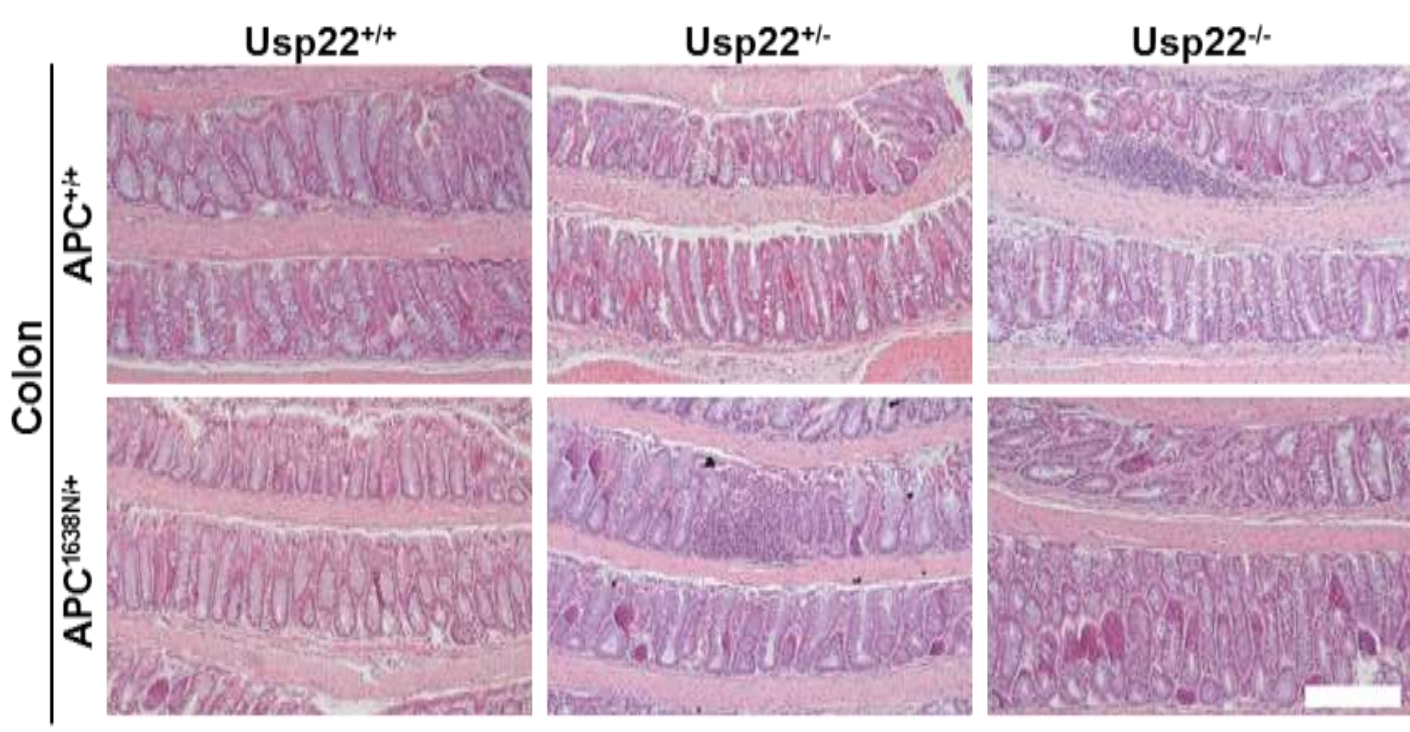

B
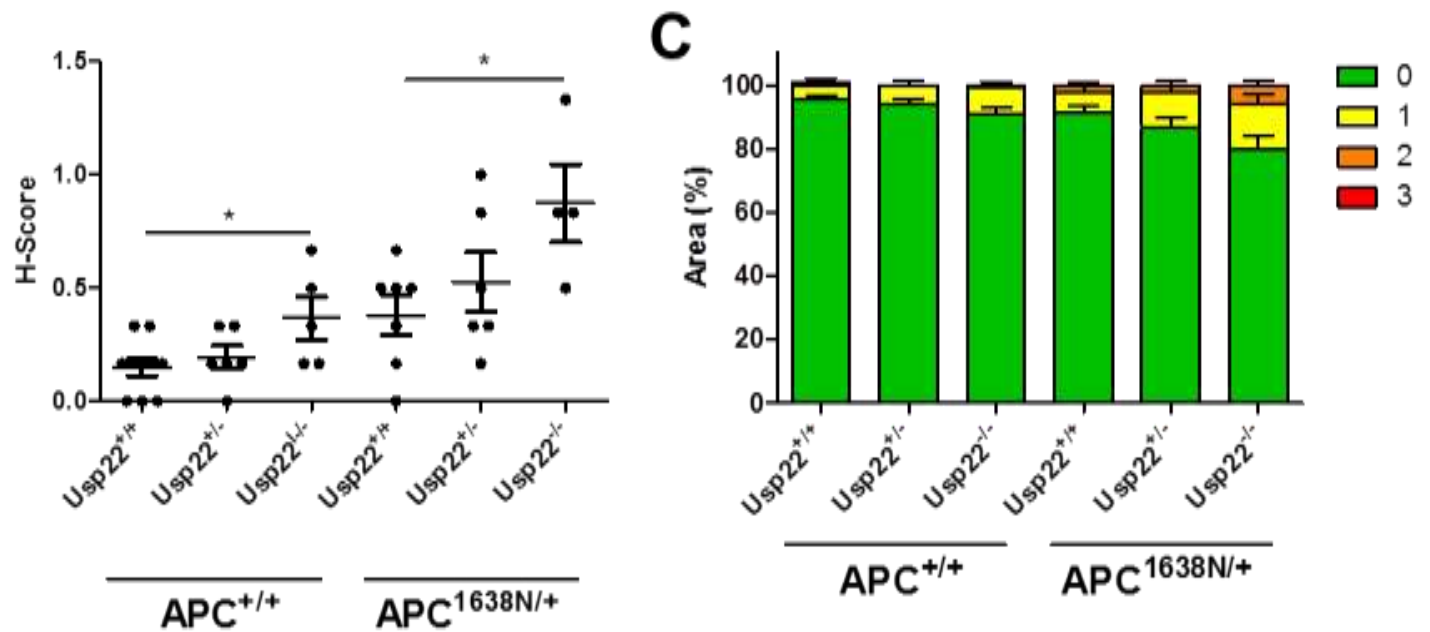

Figure 31: Usp22 ablation increases inflammation in DSS-Tam mice. (A) H\&E-stained colon sections obtained from DSS-Tam mice were assessed for signs of inflammation. Usp22 knockout mice showed increased epithelial damage and lymphocyte infiltration. Scale bar: 100 $\mu \mathrm{m}$. (B) Usp22 ${ }^{--}$animals were characterized by increased $\mathrm{H}$-scores because of (C) more severe inflammation. Mean \pm SEM, Student's $t$-test, $n$-numbers indicated in Table 17. 
Summing up, induction of colitis with DSS prior to Usp22 deletion led to a similar phenotype regarding survival, tumor number and location as in Tam-DSS mice. However, tumor and inflammation burden were less severe in DSS-Tam animals compared to Tam-DSS mice. These findings suggest that Usp22 loss prior to DSS treatment exacerbates symptoms of colitis and resulting tumor burden. 


\subsubsection{Intestinal Usp22 deletion in a model of sporadic CRC (Tam)}

\subsubsection{Intestinal Usp22 deficiency promotes sporadic colorectal tumorigenesis}

Since we could observe extreme consequences in our murine models for inflammation-induced colorectal cancer, we aimed to observe the effects of Usp22 on sporadic CRC formation (treatment group 3: Tam). Animals were injected with Tamoxifen at an age of 4 weeks and no DSS was administered. Parameters to determine the disease activity index were recorded weekly. The maximum weight loss was determined from the time point of Tamoxifen injection till sacrificing the animals and was similar in all mice. Generally, the body weight fluctuated minimally (Figure 32A). Sporadic presence of occult blood was detected in all animals and did not differ among genotypes (Figure 32B). Consistently, the disease activity index was comparable among all groups (Figure 32C). In APC ${ }^{1638 N /+}$, Usp22 $2^{-/}$only a slight increase in intestinal bleeding intensity and DAI was observed. Approximately 14-33 weeks after Tamoxifen injection APC ${ }^{1638 \mathrm{~N} /+}$, Usp22 $2^{-/}$mice died. APC ${ }^{1638 N /+}$ mice with Usp22 wild type (aged 22-64 weeks) or heterozygous state (27-71 weeks) survived significantly longer (Figure 30D). In contrast, compared to the truncated APC protein, APC wild type status clearly prolonged overall survival. In the $\mathrm{APC}^{+/+}$animals we observed that mice with depleted Usp22 had the shortest life expectancy. This observation seemed to be independent from the induction of colitis, though DSS treatment resulted in earlier lethality (Figures 18D and 28D). Similar to the DSS-treated cohort APC ${ }^{1638 \mathrm{~N} /+}$, Usp22-- mice were characterized by decreased colon lengths (Figure 32E). 

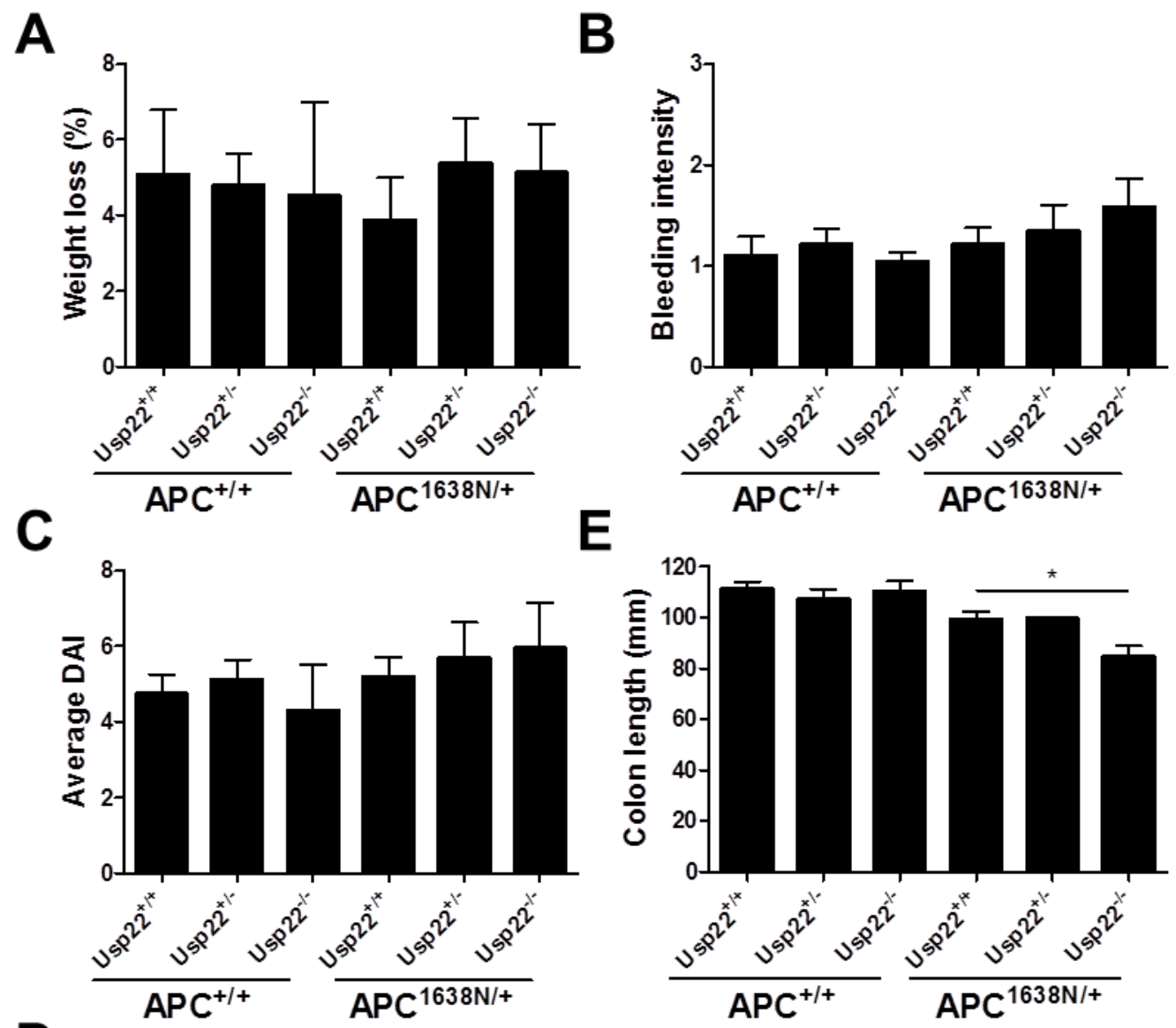

D
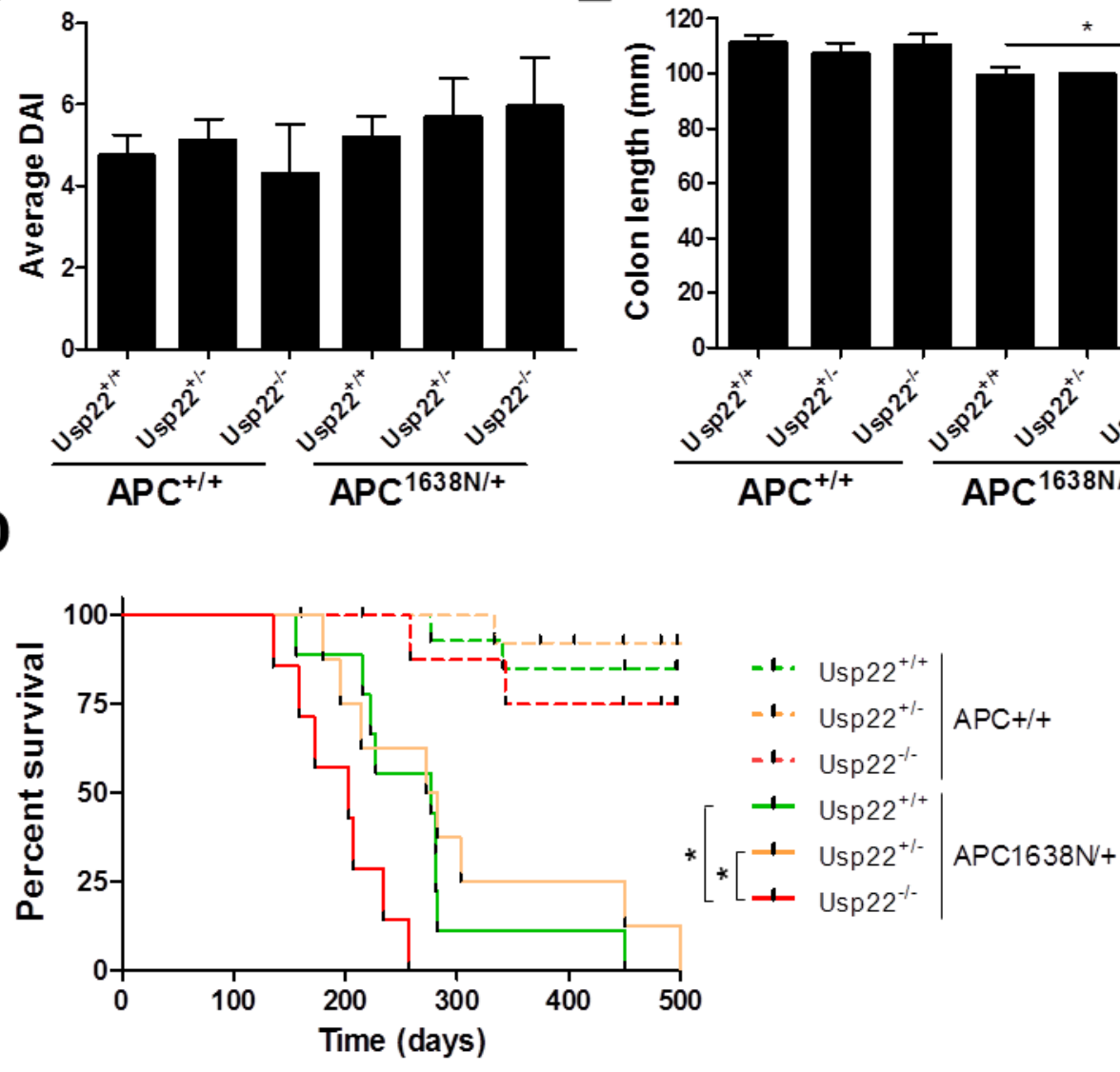

Figure 32: Usp22 loss alone results in decreased survival. (A) Weight loss, (B) bleeding intensity or (C) average DAI were barely influenced by Usp22 loss alone. (D) Upon Tamoxifen injection accelerated lethality was observed in Usp22- mice. (E) Colon length appeared to be decreased in APC ${ }^{1638 \mathrm{~N} /+}$, Usp22 $2^{-/}$animals. Mean \pm SEM, Student's $t$-test, n-numbers indicated in Table 17. 
After sacrificing the mice, we followed the same procedure as described before. Again no metastases were detected and $\mathrm{APC}^{1638 \mathrm{~N} /+}$ mice were characterized by increased spleen sizes (data not shown). As expected, the absence of colitis resulted in decreased tumor burden while the truncated APC protein in $\mathrm{APC}^{1638 \mathrm{~N} /+}$ animals correlated with promoted intestinal tumorigenesis (Figure 33A, B). On average $\mathrm{APC}^{+/+}$animals had 0-1 tumors in the colon and 58 in the SI, while 1-5 tumors were found in the colon and 15-85 in the SI of APC ${ }^{1638 \mathrm{~N} /+}$ mice. The majority of $\mathrm{APC}^{+/+}$animals with Usp22 wild type or heterozygous status was characterized by the absence of tumors in the colon while Usp22- animals possessed $0-2$ cancer lesions. In the small intestine of APC wild types the loss of Usp22 did not show any effects regarding the tumor number. In APC ${ }^{1638 N /+}$ background Usp22-- animals were characterized by significantly more oncogenic growth in the colon and SI than Usp22 $2^{+/+}$mice. Remarkably, in APC ${ }^{1638 N /+}$, Usp22- mice the tumor frequency in the small intestine was surprisingly high and comparable to the DSS-treated cohort. In the SIs of these animals 40-112 tumors were detected. Similar to the DSS-treated groups, the rate of tumors with a surface area higher than $2 \mathrm{~mm}^{2}$ was significantly increased in APC ${ }^{1638 \mathrm{~N} /+}$, Usp22 $2^{-/}$animals (Figure 33C, D). Altogether these results demonstrate that the loss of Usp22 significantly increases sporadic CRC formation even without preceding colitis. 

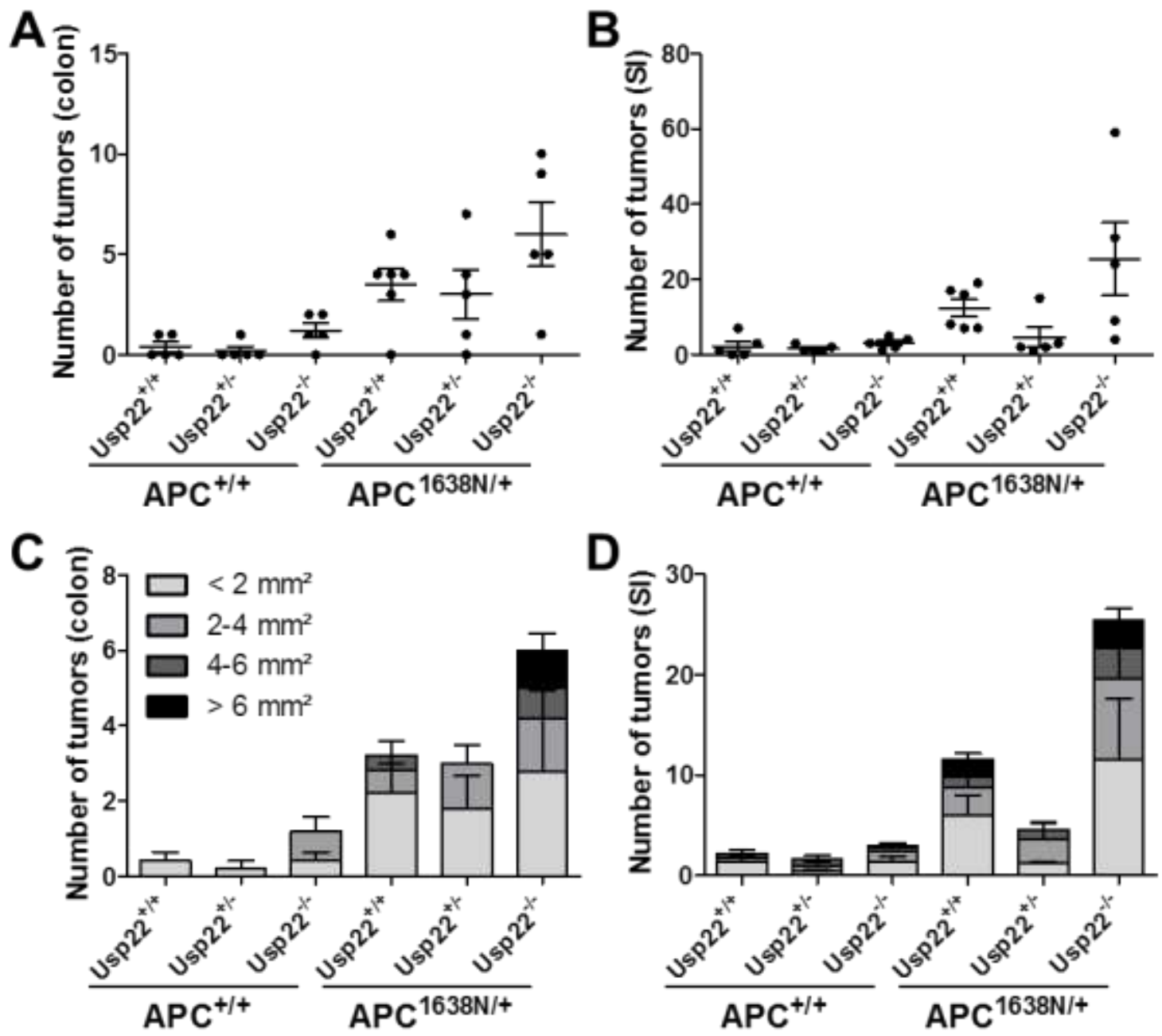

Figure 33: Usp22 loss leads to increased sporadic intestinal tumor formation. Reduced Usp22 expression, especially when combined with an APC mutation, results in increased tumor growth in (A) colon and (B) small intestine. (C, D) Partial or complete Usp22 ablation in APC ${ }^{1638 N /+}$ background results in the growth of bigger tumor lesions compared to Usp22 wild type animals. Mean \pm SEM, Student's $t$-test, n-numbers indicated in Table 17 .

Tumor location was documented and similar to the DSS-treated animals there was an accumulation of tumors in the proximal region of the small intestine in APC ${ }^{1638 \mathrm{~N} /+}$, Usp $22^{-/-}$mice. However, while tumors were mainly detected in the proximal section in the DSS-cohort, tumor growth was extended to the medial segment in case of the Tam cohort (Figure 34). In Usp22 heterozygous mice, the tumor distribution was comparable to Usp22 ${ }^{-/}$littermates. 


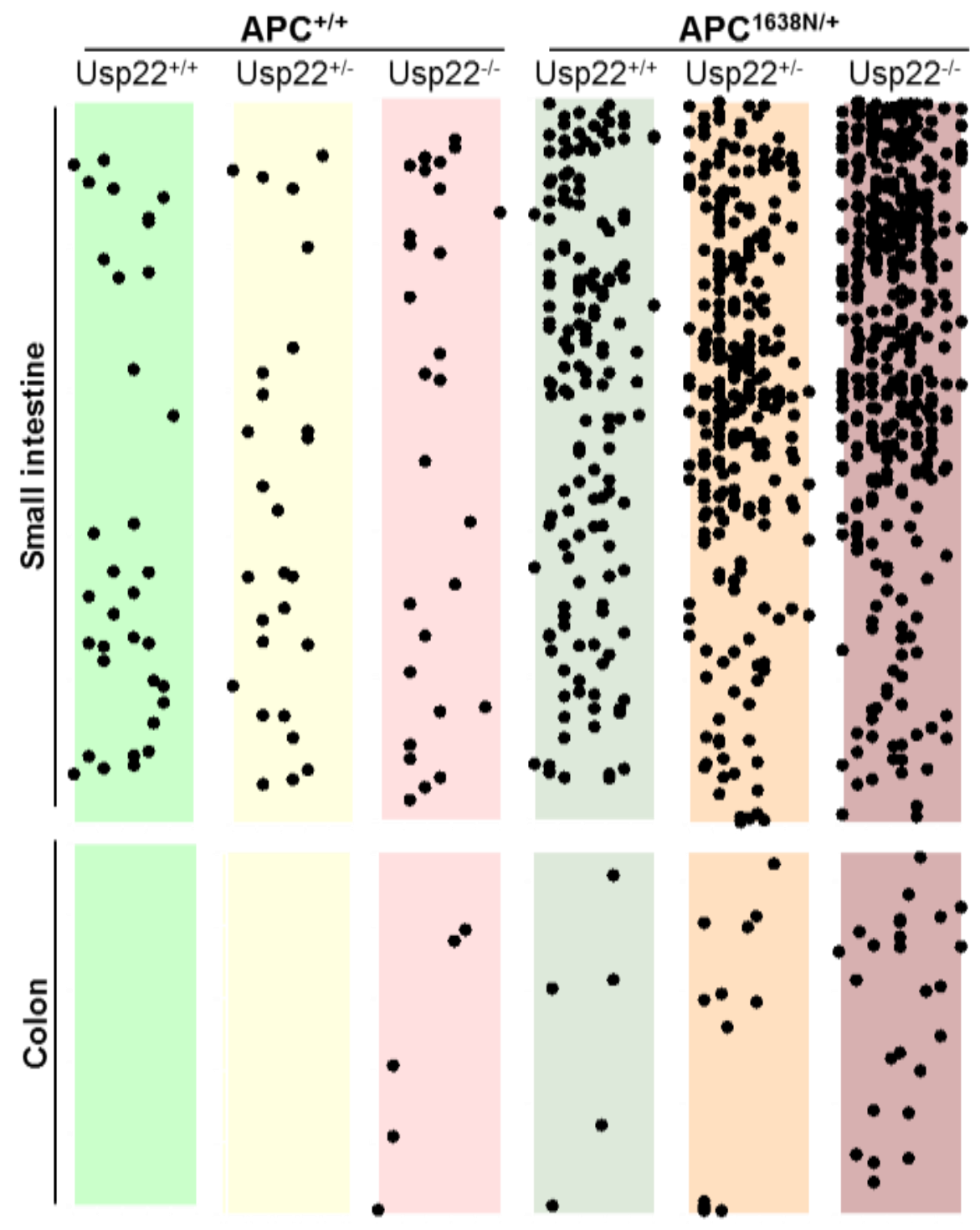

Figure 34: Sporadic CRC lesions accumulate in the proximal and medial SI region in APC $^{1638 \mathrm{~N} /+}$, Usp22 ${ }^{-/-}$mice. Relative tumor sites from five mice per genotype with representable tumor burden were depicted. Tumors were clustered in the proximal and medial segments of the small intestine of APC ${ }^{1638 N /+}$, Usp22 $2^{+/-}$and Usp22-/- animals.

Finally, we assessed on H\&E-stained colon section whether Usp22 ablation alone resulted in inflammation. Indeed, we observed several areas displaying lymphocyte infiltration and damaged epithelial sections in Usp22 ${ }^{-/}$mice (Figure 35A). Accordingly, the $\mathrm{H}$-score was elevated in these animals (Figure 35B) due to a higher percentage of inflammation-associated epithelial damage (Figure 35C). 
A

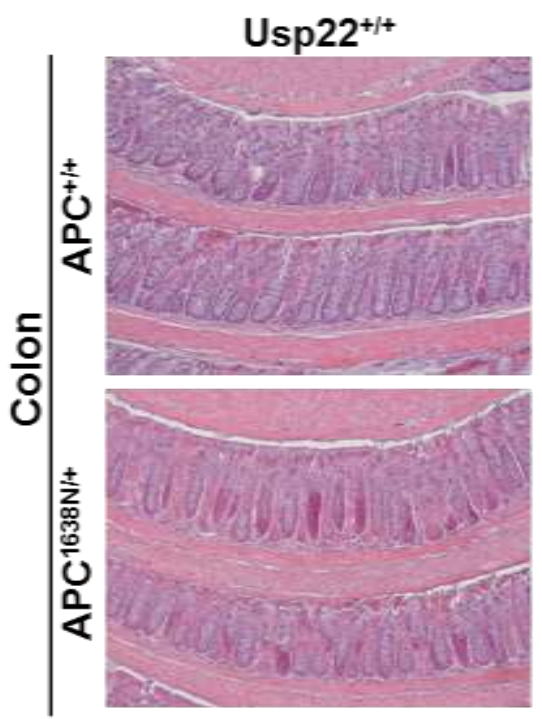

B

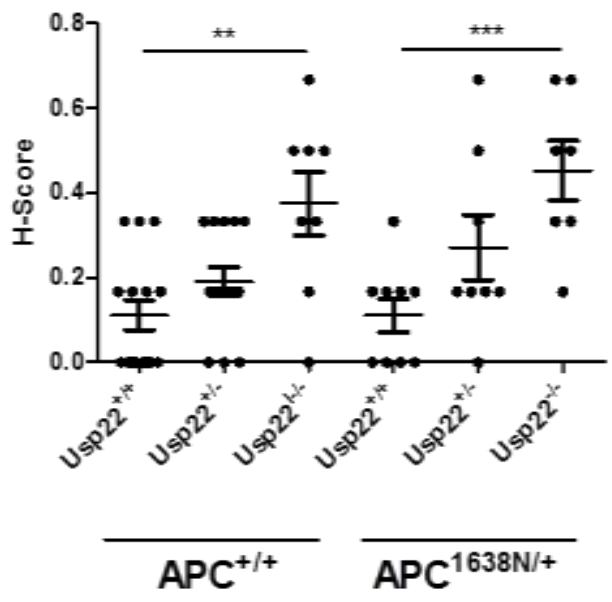

Usp22 $2^{+/-}$

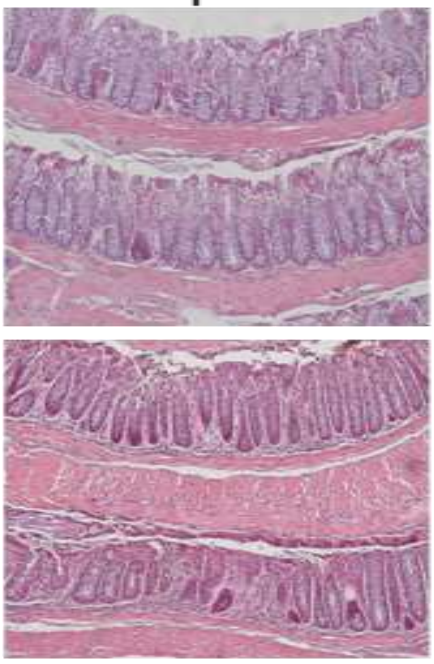

C

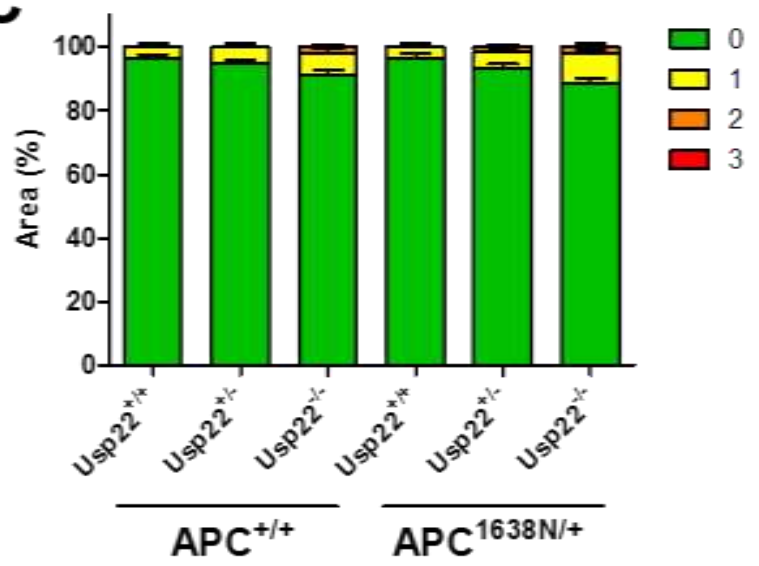

Figure 35: Usp22 loss causes mild spontaneous inflammation. (A) Intestinal Usp22 knockout without additional DSS treatment results in mild intestinal inflammation as revealed by H\&E staining on colon sections. Scale bar: $100 \mu \mathrm{m}$. (B) H-scores of Usp22- animals were slightly increased due to (C) more epithelial regions affected by inflammation-associated damage. Mean \pm SEM, Student's $t$-test, $n$-numbers indicated in Table 17.

Altogether, we could demonstrate that in APC ${ }^{1638 N /+}$, Usp22 $2^{-/}$mice, the survival is significantly decreased and tumor burden increased in a sporadic model for CRC. Moreover, Usp22 loss alone was sufficient to induce mild spontaneous inflammation. 


\subsubsection{Intestinal Usp22 deletion prior to colitis results in the worse prognosis}

After these insightful in vivo approaches, the effect of Usp22 loss in the three different treatment set-ups was to be determined in order to investigate the different aspects of the role of Usp22 in tumorigenesis. First, we investigated how a loss of Usp22 affects colitis and intestinal tumorigenesis (Tam-DSS). In the second cohort we avoided the possibility that mice with different genotypes exert differential inflammatory responses to DSS treatment, which may affect the tumor formation rate (DSS-Tam). In addition the effects of Usp22 loss alone were tested (Tam) in a model for sporadic CRC. Survival rates of Usp22 wild type, heterozygous and knockout animals were analyzed separately to emphasize the consequences of treatment options and APC status.

In mice with USP22 and APC wild type status, the survival remained mainly unaffected independent of the treatment (Figure 36A). In contrast, the truncated APC ${ }^{1638 \mathrm{~N} /+}$ protein resulted in early lethality in all mice. While life expectancies between the Tam and DSS-Tam cohorts did not differ from each other, Tamoxifen injections prior to colitis (Tam-DSS) resulted in significantly shorter survival in $\mathrm{APC}^{1638 \mathrm{~N} /+}$, Usp22 $^{+/+}$mice compared to the other APC ${ }^{1638 \mathrm{~N}}$ animals.

In Usp22 heterozygous animals, outcomes were similar, i.e. decreased life span due to APC mutation (Figure 36B). However, there was no significant difference when comparing treatment options in $\mathrm{APC}^{1638 \mathrm{~N}}$ mice.

In general, Usp22 knockout mice showed increased lethality compared to the other genotypes, irrespective of the treatment (Figure 36C). Interestingly, in APC ${ }^{1638 \mathrm{~N}}$ mice, there was hardly any difference between the Tam and DSS-Tam groups. However, compared to these two cohorts, the survival was significantly decreased in Tam-DSS mice.

In summary, in Usp22 wild type, heterozygous and knockout animals with an APC ${ }^{1638 N}$ background the Tam-DSS treatment resulted in the shortest life expectancy. The other two treatment types (Tam and DSS-Tam) led to outcomes similar to each other. 


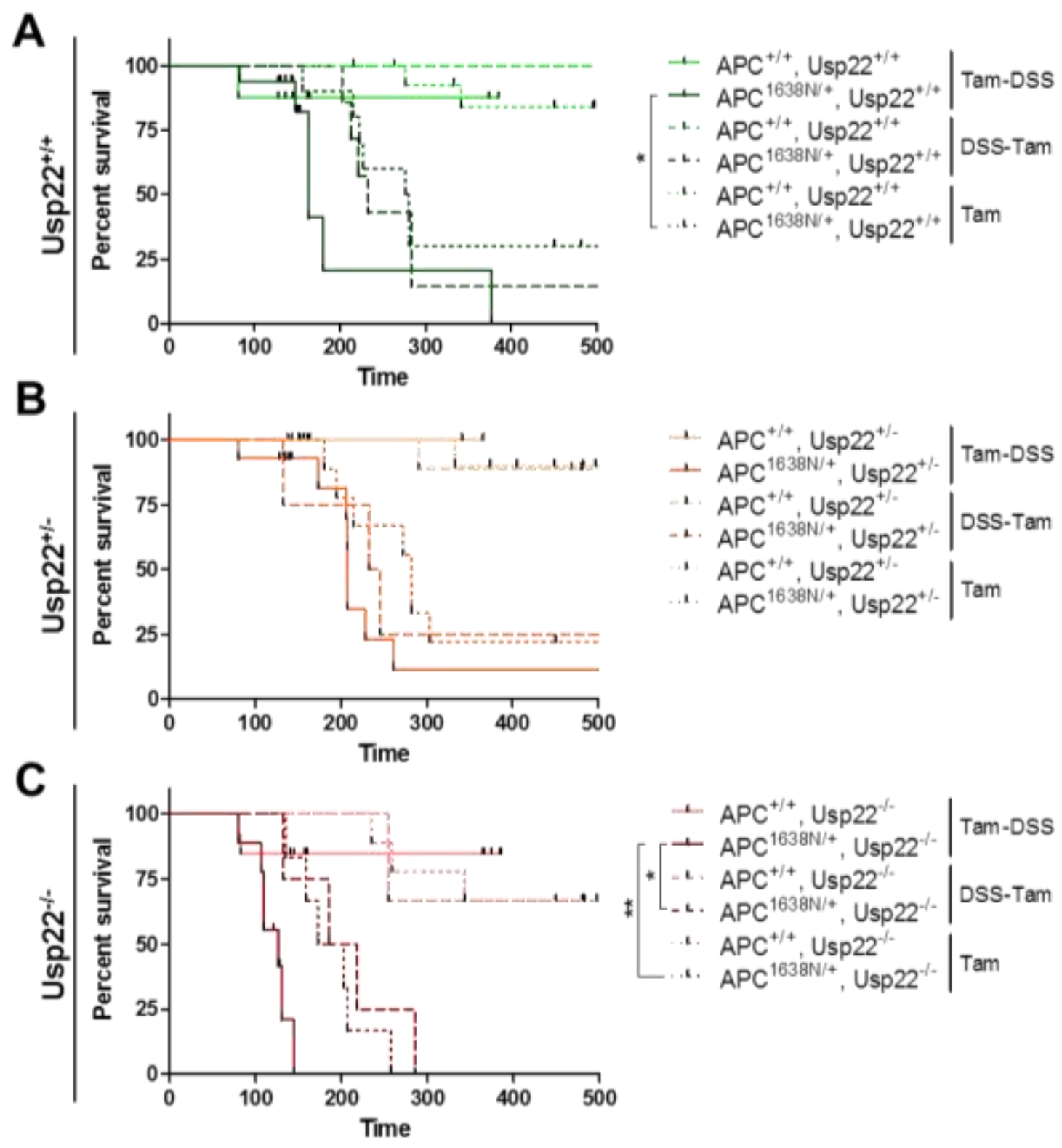

Figure 36: Survival curve analysis among treatment groups. Life expectancy of Usp22 $2^{+/+}$, Usp22 ${ }^{+/}$and Usp22 $2^{--}$animals was compared among the different treatment cohorts. (A) In all three groups (Tam-DSS, DSS-Tam, Tam) survival of Usp22 and APC wild type was hardly impaired. In Usp22 $2^{+/ 4}$, APC ${ }^{1638 \mathrm{~N} /+}$ mice the Tam-DSS group was characterized by the shortest life span. (B) In Usp22 ${ }^{+/}$animals the results were similar to the outcome of Usp22 wild type mice and no significant difference was detected among treatment groups. (C) In APC ${ }^{1638 \mathrm{~N} /}$, Usp22- mice with Tam-DSS treatment the survival was significantly shorter than in the other cohorts.

In addition to the survival analyses the tumor numbers among the three treatment groups were compared. As expected, due to the induction of colitis the oncogenic growth in the colon was 
elevated in the two DSS-treated cohorts compared to the Tam group (Figure 37A, B). Notably, the Tam-DSS group possessed most tumors and their burden was on average 2 to 3 -fold higher than in DSS-Tam mice. In contrast, oncogenic growth in colons of Tam animals was lowest; however, tumor numbers did not differ significantly from the DSS-Tam cohort (Figure 37C, D). Surprisingly, when observing tumor growth in the small intestine, we observed that the majority of Tam animals possessed even more tumors than the Tam-DSS animals. In this comparison several key observations were made. Firstly, when deleting Usp22 prior to inducing colitis (Tam-DSS), the tumor burden was significantly higher than when performing the knockout after DSS treatment (DSS-Tam). Second, in the Tam group the loss of Usp22 alone was sufficient to result in tumor formation, especially in the background of an APC mutation. Finally, as expected (Adams and Bornemann, 2013), DSS treatment promoted tumor formation exclusively in the colon while the small intestine was not or only marginally affected by colitis. Together, our in vivo experiments to investigate the role of Usp22 loss in intestinal tumorigenesis revealed that surprisingly, Usp22 loss decreased survival and increased tumor number. Moreover, oncogenic burden was highest in the Tam-DSS cohort suggesting the interplay of Usp22 ablation and inflammatory processes within the intestine. 

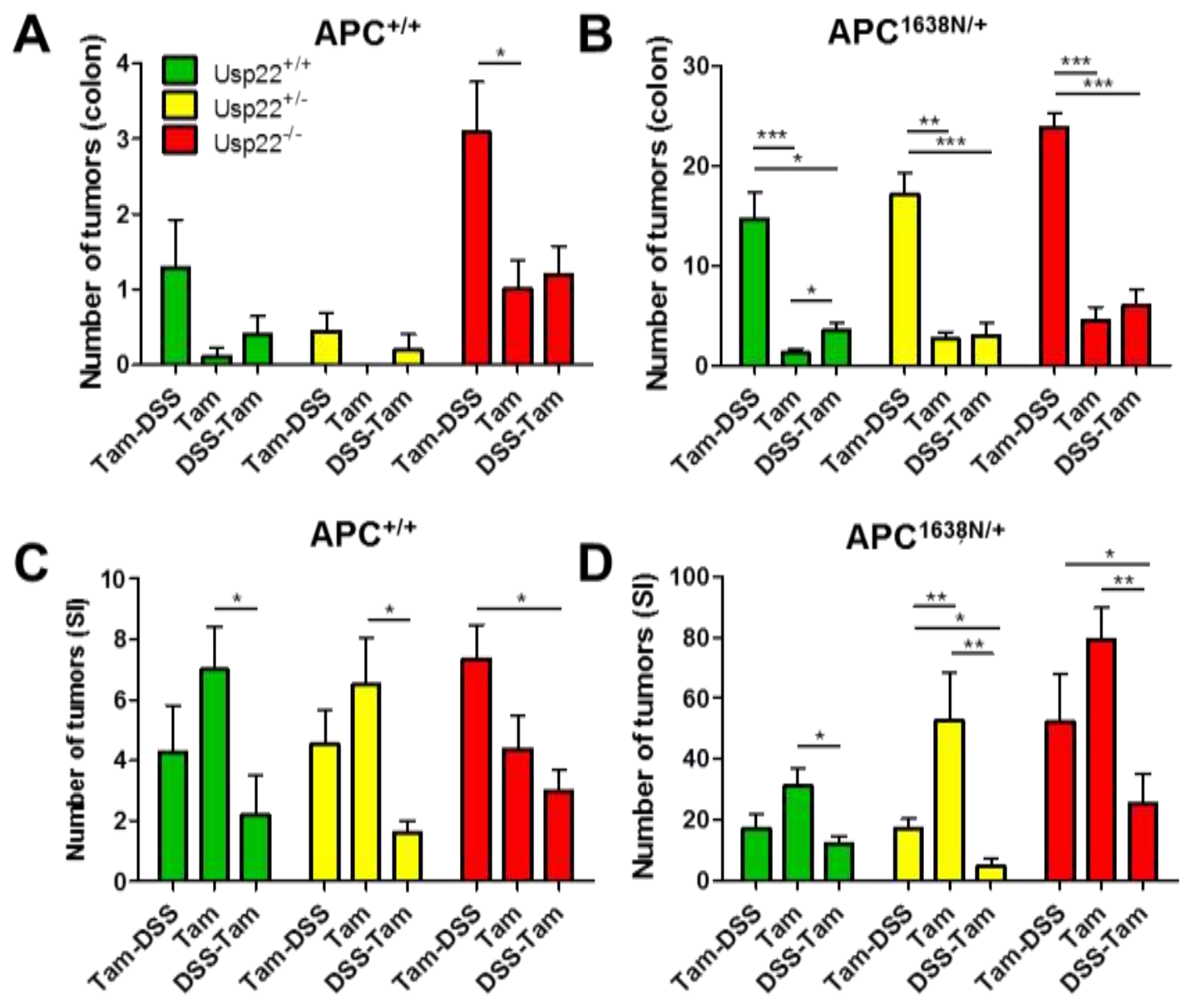

Figure 37: Comparison of tumor burden among treatment groups. Tumor numbers in colon and small intestine were compared in Usp22 $2^{++}$, Usp22 $2^{+/-}$and Usp22 $2^{--}$among the different treatment cohorts. (A, B) In the colon the Tam-DSS group was, irrespective of the genotype, characterized by a significantly higher tumor burden than the other two cohorts. Differences between Tam and DSS-Tam groups were only marginal in the colon. (C, D) In the small intestine, Tam mice possessed most tumors; only $\mathrm{APC}^{+/+}$, Usp22 $2^{-/-}$mice were affected more severely by Tam-DSS. Mean \pm SEM, Student's $t$-test, n-numbers indicated in Table 17 . 


\subsection{Intestinal Usp22 loss elevates acute colitis burden resulting in bone fragility}

In our previous DSS experiments we could observe severe inflammatory reactions in Usp22-animals even several weeks after the end of the treatment. Moreover, our results indicated a potential interplay between ablated Usp22 levels, increased inflammation and therefore elevated intestinal tumorigenesis. Since there were still inflammatory reactions long time after terminating DSS treatment, we assumed that these symptoms are even stronger when the colitis is highly acute. Thus, we aimed to observe the consequences of Usp22 during the acute phase of intestinal inflammation. For this purpose, we induced colitis in small mouse cohorts (Table 18) and sacrificed these animals two days after terminating the DSS treatment. To determine the disease activity index, stool consistency, Guaiac score and body weight were recorded daily. We could observe diarrhea and the presence of occult blood more frequently after the loss of Usp22 than in wild type animals (Figure 38A, B). Moreover, these parameters were also worsened in APC mutant compared to $\mathrm{APC}^{+/+}$mice. While the weight loss was not significantly changed among the groups (data not shown), clear differences were detected in the DAI (Figure 38C, D). The disease activity index increased in a similar rate in all groups, however, the burden was highest in $\mathrm{APC}^{1638 \mathrm{~N} /+}$, Usp22 $2^{-/-}$animals.

Table 18: Sizes of the experimental mouse cohorts in which the effects of Usp22 ablation was observed during acute colitis.

\begin{tabular}{|c|c|c|c|c|}
\hline & \multicolumn{2}{|c|}{$\mathrm{APC}^{+/+}$} & \multicolumn{2}{|c|}{ APC $^{1638 N /+}$} \\
\hline & Usp22 $2^{+/+}$ & Usp22-/- & Usp22 $^{+/+}$ & Usp22 ${ }^{-1}$ \\
\hline Acute colitis & 6 & 4 & 3 & 3 \\
\hline
\end{tabular}


A

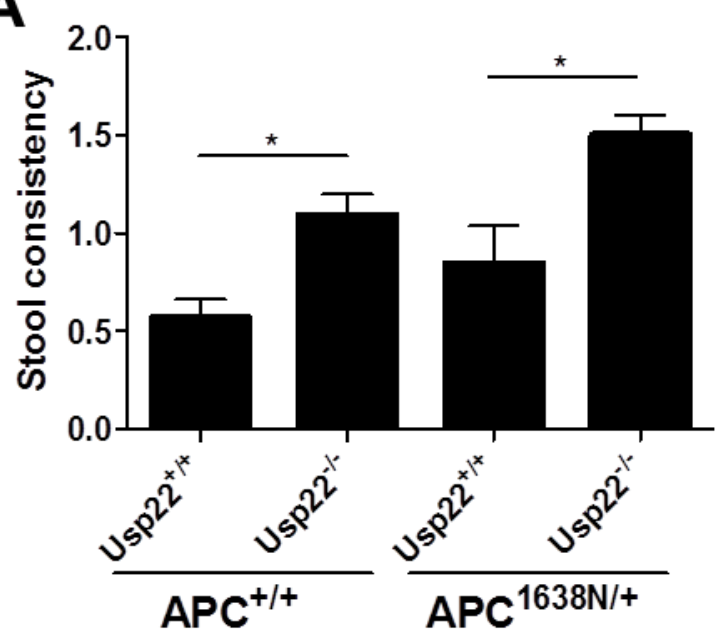

C

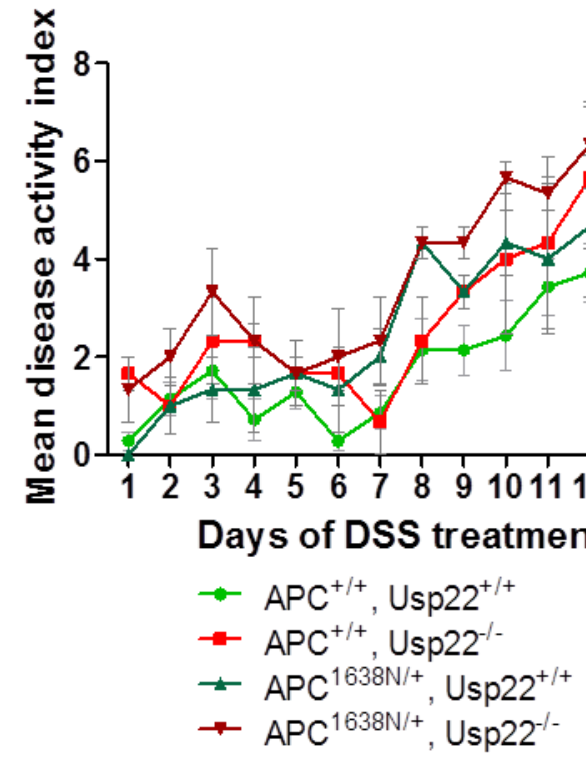

B

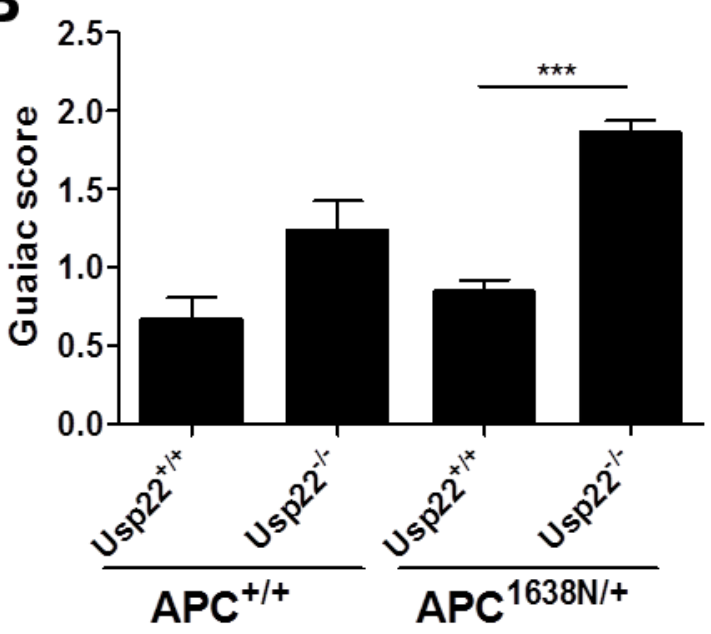

D

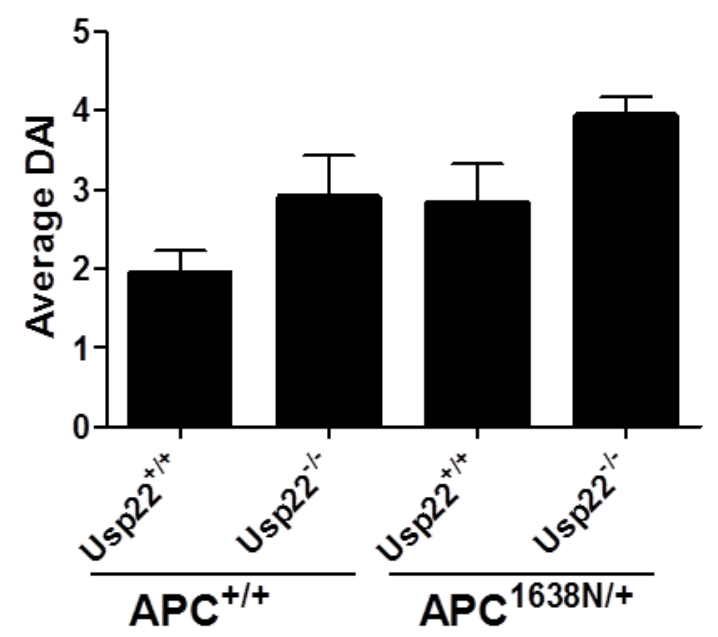

Figure 38: Acute colitis is correlated with increased DAI in Usp22-/-mice. During DSS administration (A) stool consistency and (B) bleeding intensity were recorded to determine the disease activity index. Stool was softer and bloodier in Usp22 $2^{--}$mice. (C) The daily calculated disease activity index rose similarly in all mice (D) but the average values of the entire time period was highest in APC ${ }^{1638 \mathrm{~N} /+}$, Usp22 $2^{-/}$animals. Mean \pm SEM, Student's $t$-test, n-numbers indicated in Table 18. 
After sacrificing animals, blood was extracted and inner organs were isolated. A striking difference was observed while comparing APC wild type and mutant mice to each other. When extracting blood we noticed that it was paler in $\mathrm{APC}^{1638 \mathrm{~N} /+}$ mice. Thus, we determined the ratio between hematocrit and serum. In APC wild types we could measure between 55-60\% hematocrit in a total blood sample while the ratio was approximately $48 \%$ in $\mathrm{APC}^{1638 \mathrm{~N} /+}$, Usp22 $^{+/+}$and 38\% in APC ${ }^{1638 N /+}$, Usp22 $2^{-/}$mice (Figure 39A). Accordingly, kidneys (Figure 39B) and livers (Figure 39C) were extremely pale in these animals.

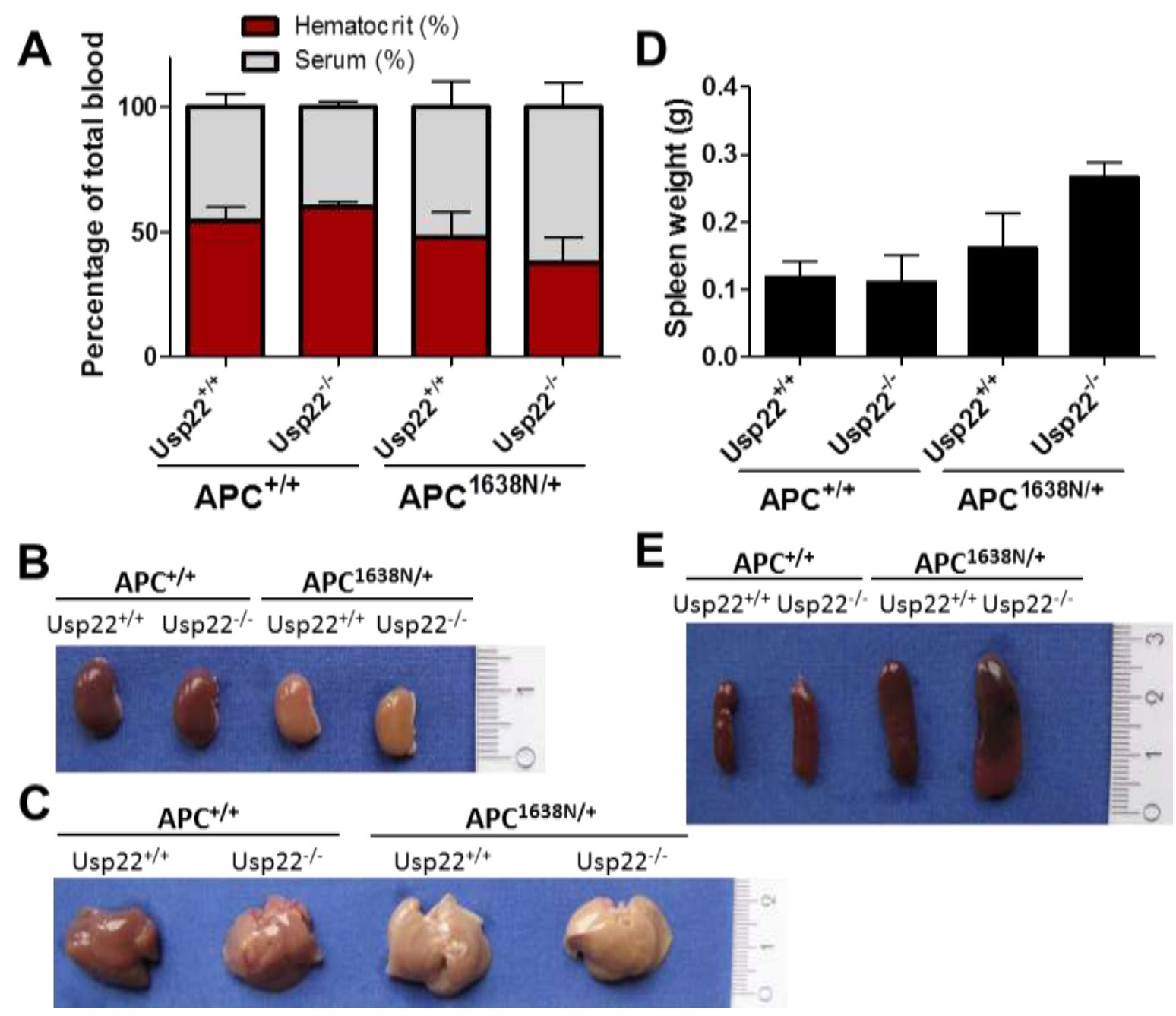

Figure 39: The anemic effect of an APC mutation is slightly aggravated by Usp22 loss. The relative amount of hematocrit was lower in blood samples of APC ${ }^{1638 \mathrm{~N} /+}$ mice, especially after Usp22 loss. (B) Livers and (C) kidneys were pale in APC mutated animals while (D, E) their spleen was enlarged and heavier. Mean \pm SEM, Student's $t$-test, $n$-numbers indicated in Table 18. 
In accordance with Qadri and colleagues (Qadri et al., 2012) spleens were enlarged in mice carrying a heterozygous mutation in the APC gene (Figure 39D, E). Interestingly, spleens of $\mathrm{APC}^{1638 \mathrm{~N} /+}$ animals were, although not significant, even bigger after the loss of Usp22.

As expected, the acute inflammatory reaction had an effect on the length of the intestinal organs. While the colon length was similar for the majority of animals, APC ${ }^{1638 \mathrm{~N} /+}$, Usp22 $2^{-/-}$mice were characterized by shorter colons (Figure 40A, B). In the small intestine only mild effects were observed. On average, SIs of $\mathrm{APC}^{1638 \mathrm{~N} /+}$, Usp22 $2^{-/-}$were slightly shorter; however, this difference was not significant (Figure 40C).
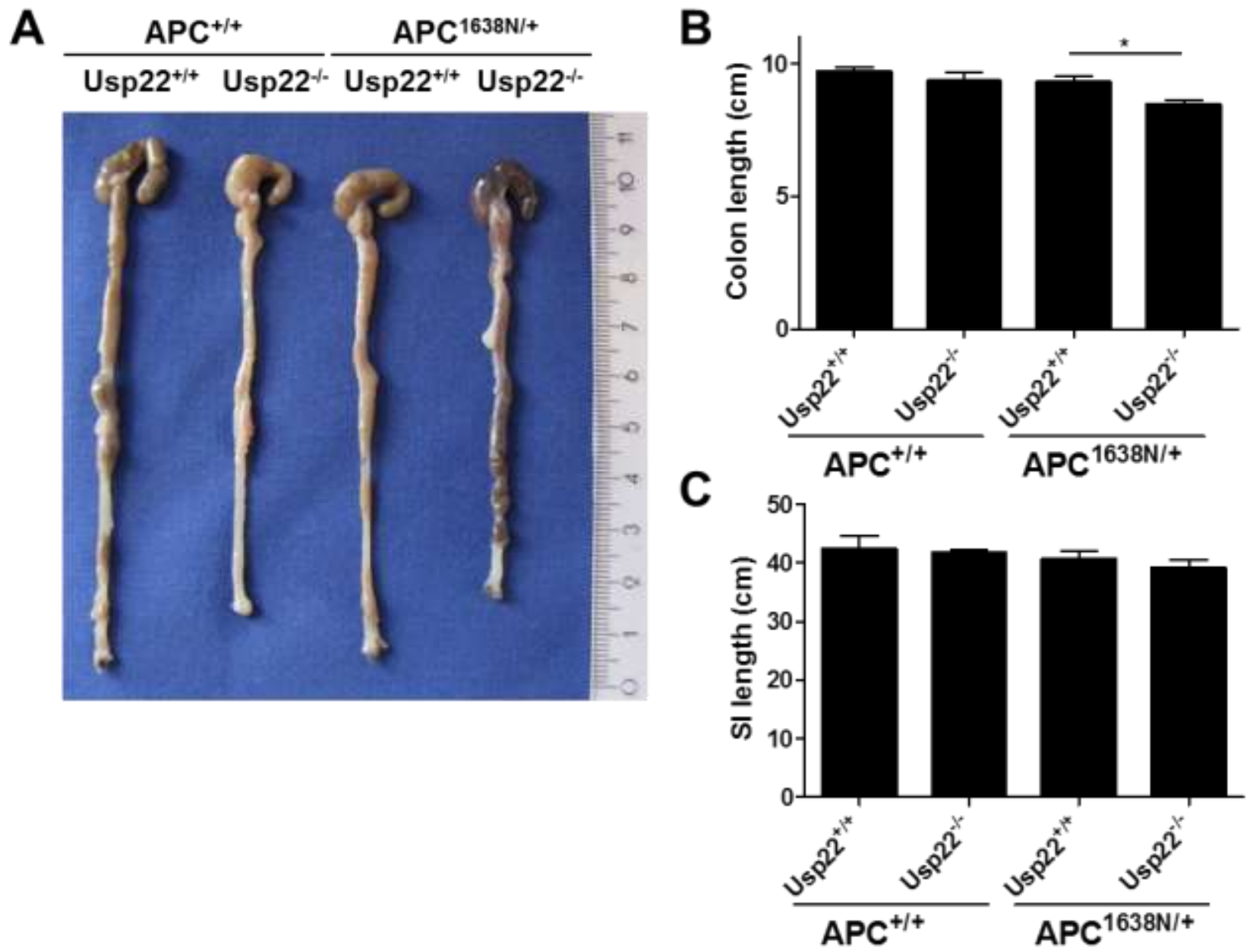

Figure 40: Acute colitis results in decreased colon lengths in APC ${ }^{1638 N /+}$, Usp22 ${ }^{-/-}$animals. $(A, B)$ Colons and $(C)$ small intestines were measured after sacrificing animals. Colons were slightly shorter in APC ${ }^{1638 \mathrm{~N} /+}$, Usp22 $2^{-/-}$mice while the small intestines were largely unaffected. Mean \pm SEM, Student's $t$-test, n-numbers indicated in Table 18 . 
Colons were stained with $\mathrm{H} \& \mathrm{E}$ to assess the overall organ morphology and the inflammation burden. As expected, acute colitis led to inflammation-associated epithelial damage in all animals (Figure $41 \mathrm{~A})$.
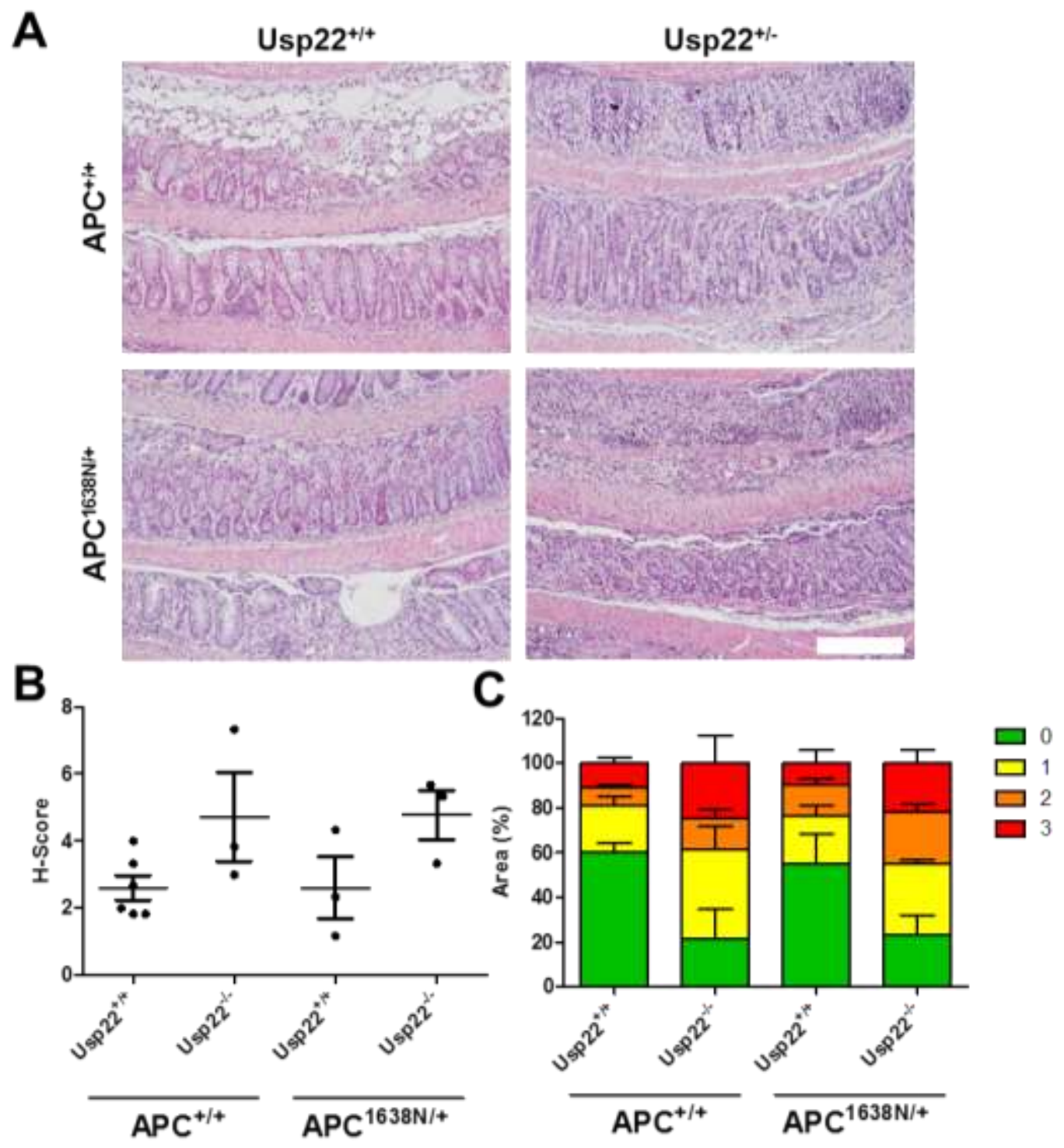

Figure 41: Intestinal loss of Usp22 increases burden during acute colitis. (A) Two days after terminating the DSS administration, mice were dissected and colons were stained with $\mathrm{H} \& \mathrm{E}$. While in all animals epithelial integrity was disturbed, in Usp22 ${ }^{-1-}$ mice more colon segments were characterized by a complete absence of crypt structures. Scale bar: $100 \mu \mathrm{m}$. (B) The average $\mathrm{H}$-score was elevated upon the loss of Usp22 and accordingly (C) more colon segments were affected by severe inflammation-induced epithelial damage in these mice. Mean \pm SEM, Student's $t$-test, $n$-numbers indicated in Table 18 . 
In Usp22 $2^{+/+}$mice several organ regions were detected in which the integrity of the epithelium was disturbed. However, in the majority of colon regions only mild signs of inflammation were observed. In contrast, Usp22-/- animals were characterized by severe epithelial damage and in large segments of the organ no crypt structures were detected at all. In the minority of tissue, normal crypts could be found. Accordingly, the average $\mathrm{H}$-score (Figure 41B) and the percentage of severely damaged tissue was elevated upon the loss of Usp22 (Figure 41C). These findings were independent from the APC status of experimental animals.
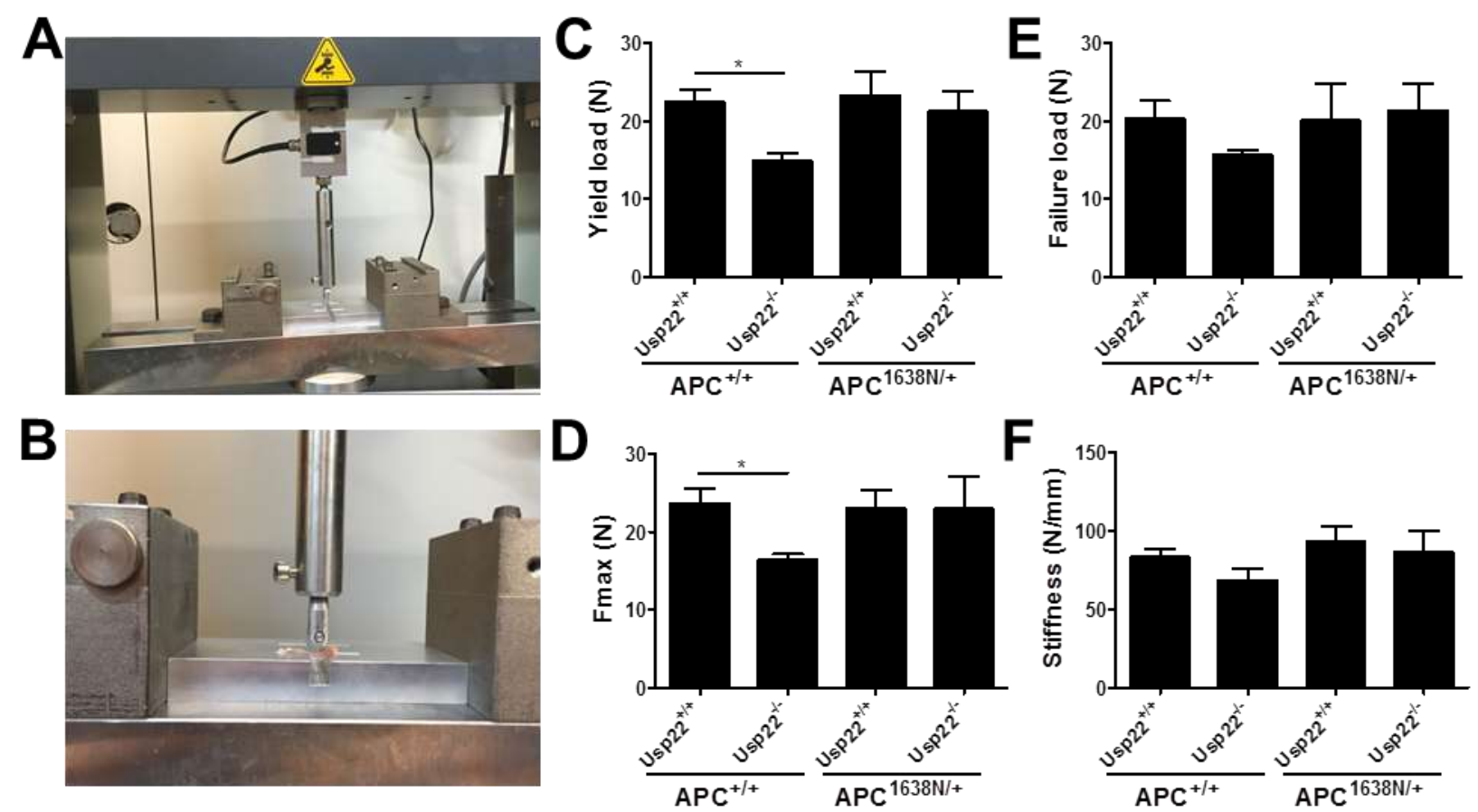

Figure 42: Acute colitis results in increased bone fragility in APC ${ }^{+/+}$, Usp22 ${ }^{-/-}$mice. (A, B) To determine bone fragility, femora were placed into a Zwick device which applied pressure onto the bone leading to fracture. The loss of Usp22 did not show any effect in APC ${ }^{1638 \mathrm{~N} /+}$ mice with regards to $(C)$ yield load, (D) Fmax, (E) failure load and (F) stiffness. However, these parameters revealed that bones of $\mathrm{APC}^{+/+}$, Usp22 $2^{-/}$mice are significantly more fragile than femora of Usp22 wild types. Mean \pm SEM, Student's $t$-test, n-numbers indicated in Table 18.

Generally, it was observed in IBD patients that intestinal inflammation correlated with decreased bone mineral density and increased fracture risk (Ali et al., 2009; Bernstein et al., 2000). To determine whether the loss of Usp22 and acute colitis have an impact on bones, we observed the bone fragility. Using a Zwick device (Figure 42A, B), the biomechanical properties 
of femora were measured in cooperation with Dr. med. Dominik Saul (Department of Trauma, Orthopedics and Reconstructive Surgery, UMG). With these analyses the applied strength during bone deformation (Figure 42C; yield load) and bone fracture (Figure 42D; Fmax) was determined. Moreover, the maximum forces applied before breaking the femora (Figure 42E; failure load) and the bone stiffness (Figure $42 \mathrm{~F}$ ) were quantified. In $\mathrm{APC}^{1638 \mathrm{~N} /+}$ mice, no significant difference was detected between Usp22 $2^{+/+}$and Usp22 $2^{-{ }^{-}}$animals. Interestingly, the bone stiffness was significantly decreased in $\mathrm{APC}^{+/+}$, Usp22 $2^{-/-}$mice compared to their Usp22 wild type littermates. Interestingly, inflammatory reactions were sufficient to show an effect on bone fragility in $\mathrm{APC}^{+/+}$but not $\mathrm{APC}^{1638 \mathrm{~N} /+}$ animals with a homozygous Usp22 deletion. In summary, these data reveal that effects of acute colitis are aggravated in Usp22 $2^{-/-}$mice. 


\subsection{Heterogeneous USP22 expression levels in CRC patients and cell lines}

Previous studies on human material described that high USP22 expression correlates with increased oncogenic growth, distant metastasis and poor prognosis (Melo-Cardenas et al., 2016). Unexpectedly, we showed that abolishing Usp22 expression in the context of inflammation-induced and sporadic colorectal cancer in vivo resulted in highly increased tumor numbers with an aggressive phenotype. In order to obtain insights into the mechanisms responsible for these phenomena, we performed several in vitro and in silico experimental approaches. We started our investigations with the analysis of publically available cancer databases. Using the IST Online ${ }^{\circledR}$ database possessing expression data from cancer patients we observed that a high fraction of CRC patients has low USP22 levels (Figure 43A). Moreover, there was no clear correlation between survival rates of patients with low or high USP22 expression, respectively. This finding was surprising since it contradicted most published reports. However, these data reflected the situation for colorectal cancer in general, thus, more detailed records were obtained from the Oncomine ${ }^{\mathrm{TM}}$ platform (TCGA Colorectal Statistics, reporter AB028986_1_4358). These data enabled us to identify USP22 mRNA levels in different types of cancer in cecum, colon or rectum as well as in normal control samples. Notably, mean expression values of all depicted cancer types were lower than in the control (Figure 43B).

To investigate the molecular aspects underlying the effects of USP22 in tumorigenesis we planned to perform experiments in vitro. For this purpose protein lysates were isolated from several human colorectal cancer cell lines. Afterwards, global USP22 protein levels were determined in these rectal adenocarcinoma, colorectal adenocarcinoma and carcinoma cells by western blot. As expected, USP22 levels were as diverse as seen in CRC patient data obtained from the databases (Figure 43C). While USP22 was detected in all cell lines, only faint protein bands were detected in $\mathrm{NCl}-\mathrm{H} 508$ and T84 cells. Protein amounts were highest in COLO320DM, RKO, SW48 and SW480 cells. 


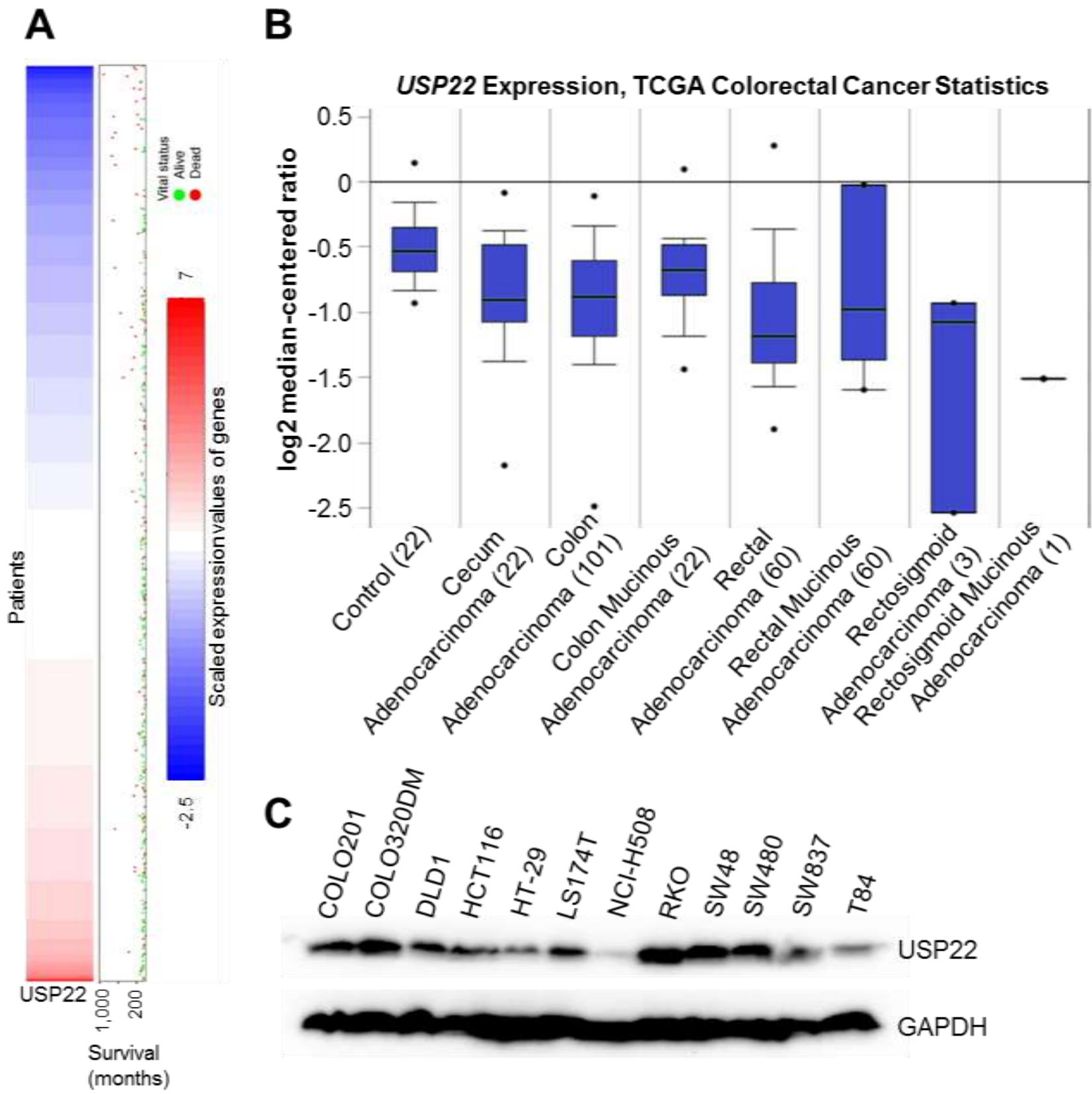

Figure 43: USP22 expression is heterogeneous in CRC patient samples. (A) USP22 expression is not only upregulated as reported in literature but was found to be downregulated in approximately $50 \%$ of CRC patients (obtained from the IST Online ${ }^{\circledast}$ database). (B) Oncomine $^{\mathrm{TM}}$ data (TCGA Colorectal Statistics, reporter AB028986_1_4358) reveal reduced USP22 mRNA levels in cecal and several colon- and rectum-associated cancers. (C) USP22 protein levels are highly diverse in the twelve tested colorectal cancer cell lines. 
In summary, in CRC patients USP22 mRNA expression levels were not as "black and white" as described in the literature. In fact, a high fraction of colorectal cancer patients is characterized by low USP22 levels resembling our in vivo results well. This heterogeneity could also be confirmed on protein levels in diverse human CRC cell lines we tested by western blot. Since to our surprise USP22 expression was seen to be highly diverse in human CRC samples, we aimed to further analyze the incidence of patients with low USP22 levels. For this purpose patients with heterozygous deletions of the USP22 gene were identified using the cBioPortal for Cancer Genomics. Astonishingly, in some cancer types, the majority of patients are affected. In fact, approximately $45-50 \%$ of colorectal cancer patients have heterozygous USP22 deletions (Figure 44A). This finding underlines the relevance of elucidating the consequences of USP22 loss in colorectal cancer. Therefore, we decided to perform in vitro analyses with a siRNA-mediated loss of USP22. SW480 CRC cells were transfected with four individual siRNAs targeting USP22 (siUsp22) and with a smart pool (SP) containing a combination of these siRNAs. A non-targeting siRNA (siControl) was used as a control. All transfections resulted in a significant decrease of USP22 mRNA levels (Figure 44B). After verifying its efficiency on protein level (Figure 44C) we decided to use the smart pool (SP) for all subsequent in vitro experiments. 


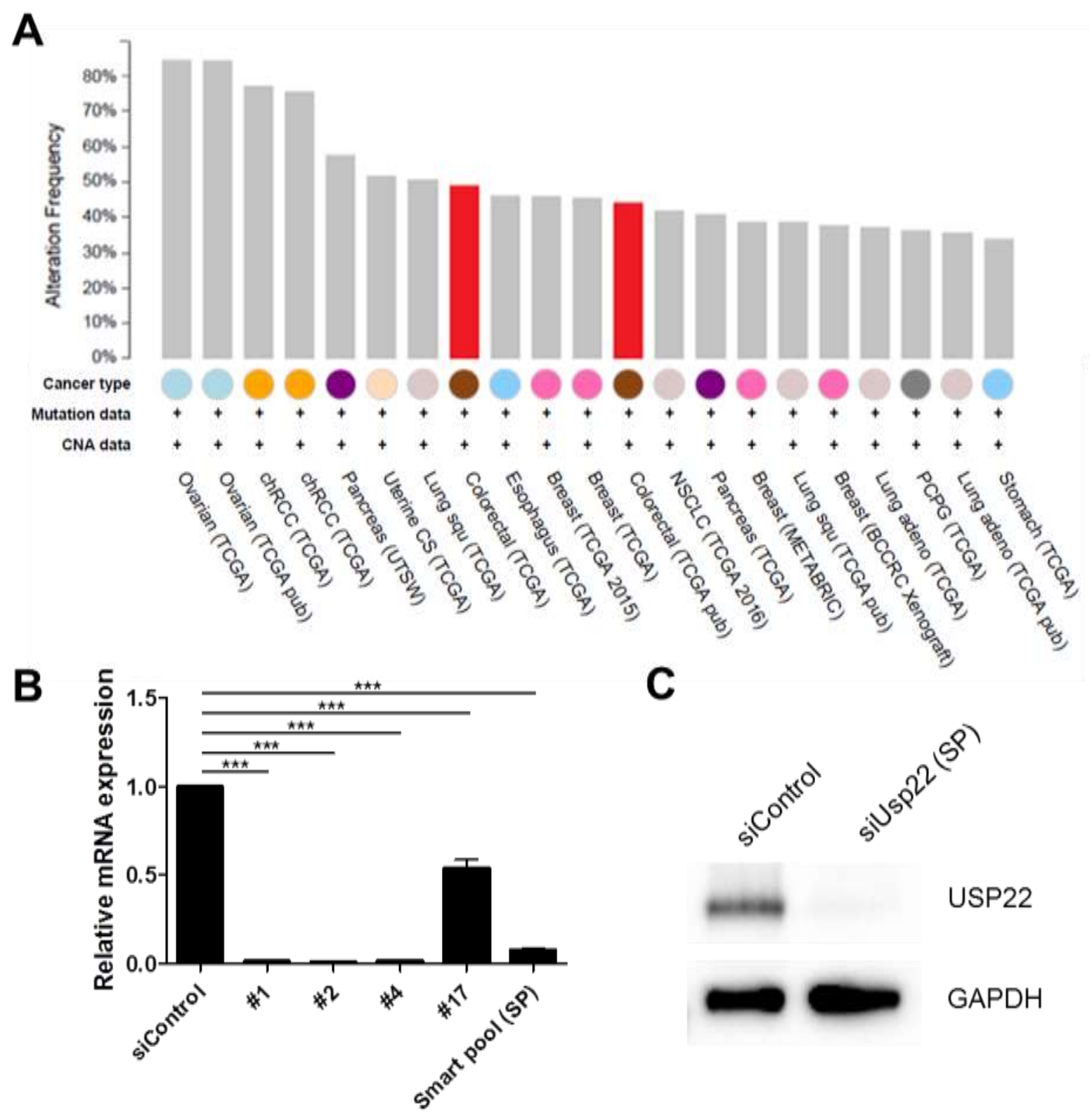

Figure 44: Heterozygous USP22 deletions occur frequently in CRC patients and can be simulated by siRNA treatment in vitro. (A) Data obtained from the cBioPortal for Cancer Genomics website reveal that a big percentage of cancer patients is characterized by a heterozygous deletion of USP22 (red: CRC data sets; HETLOSS function). (B) To simulate the loss of USP22 in vitro, different individual siRNAs targeting USP22 (siUsp22) and a smart pool (SP) were tested for their knockdown efficiency on mRNA level in SW480 cells. Expression values were normalized to a non-targeting control siRNA (siControl). Mean \pm SD, Student's $t$ test, $n=3$. (C) The smart pool results in a strong reduction of USP22 on protein level in SW480 cells. 
Since altered USP22 expression levels were described to affect tumorigenesis, we expected that USP22 would have an effect on the morphology or growth rate of human colorectal cancer cells. We knocked down USP22 in nine CRC cell lines and observed their morphology after 72 hours. No striking differences were detected when comparing USP22 knockdown and control cells with regards to cell size, shape, number of nuclei etc. (Figure 45).

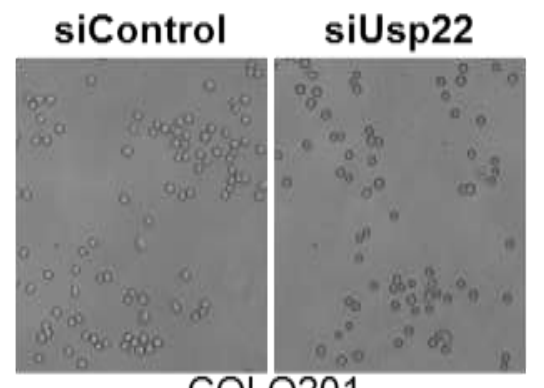

COLO201

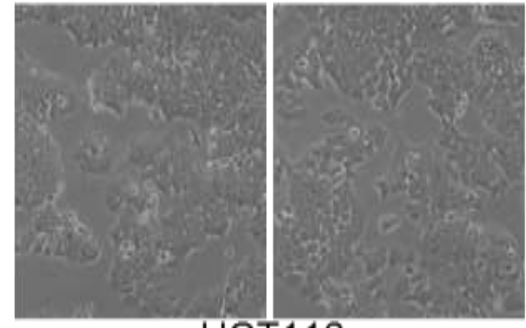

HCT116

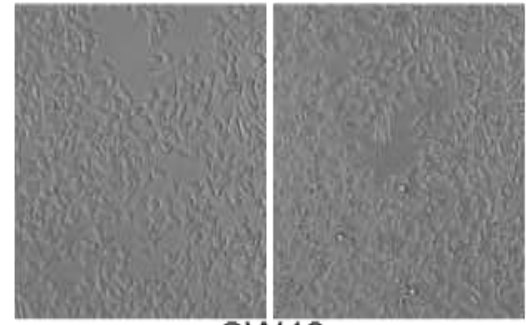

SW48

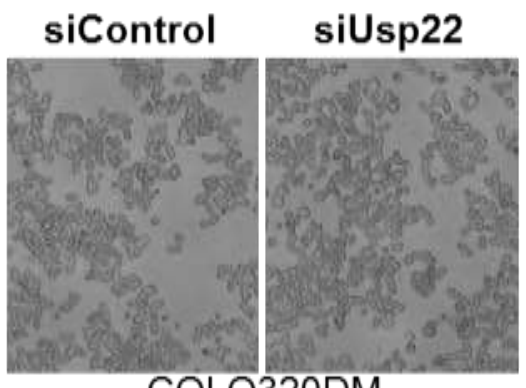

COLO320DM

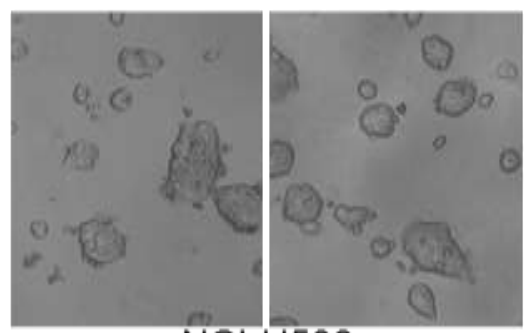

$\mathrm{NCl}-\mathrm{H} 508$

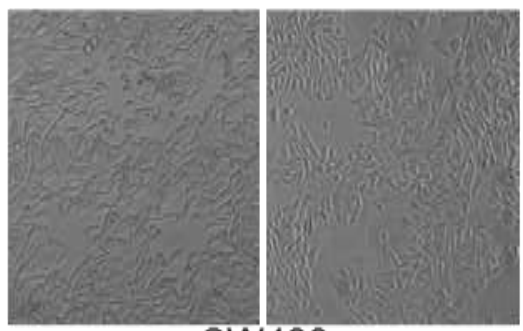

SW480
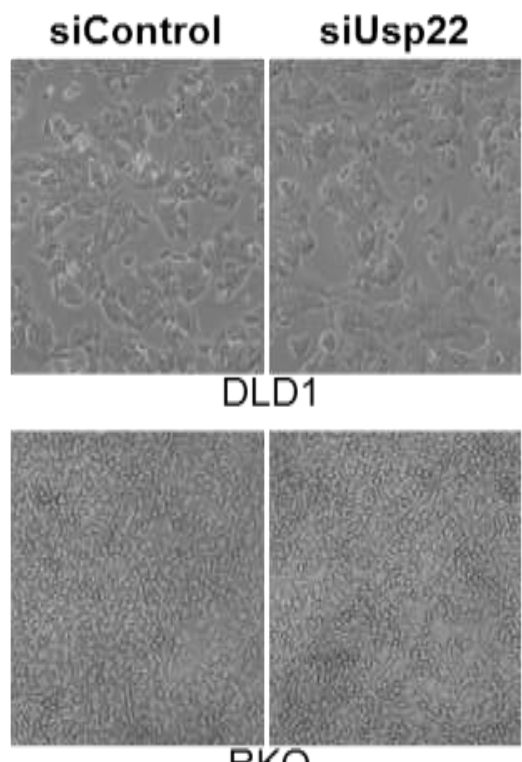

RKO

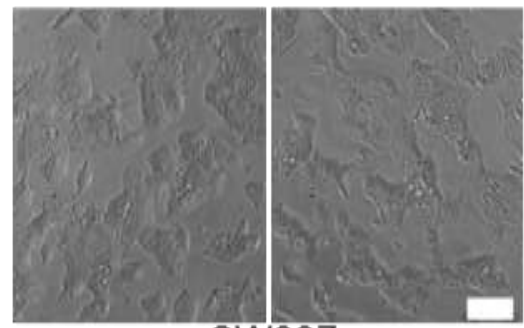

SW837

Figure 45: Knockdown of USP22 marginally affects the morphology of human CRC cell lines. 72 hours after the siRNA-mediated loss of USP22, the morphology was assessed in nine cell lines. No or only marginal effects were observed. Scale bar: 1,000 $\mu \mathrm{m}$.

Next, we compared the proliferation rates of these cell lines upon siRNA-mediated USP22 knockdown over a time period of one week using the Celigo ${ }^{\circledR}$ device. We could observe that the loss of USP22, depending on the cell line, can have varying effects on cell growth (Figure 46). While no difference was detected in NCl-H508, SW480 and SW837 cells, proliferation 
levels were reduced upon USP22 knockdown in the majority of cells, i.e. COLO201, COLO320DM, DLD1, RKO and SW48. Interestingly, cell growth was significantly increased in HCT116 cells.
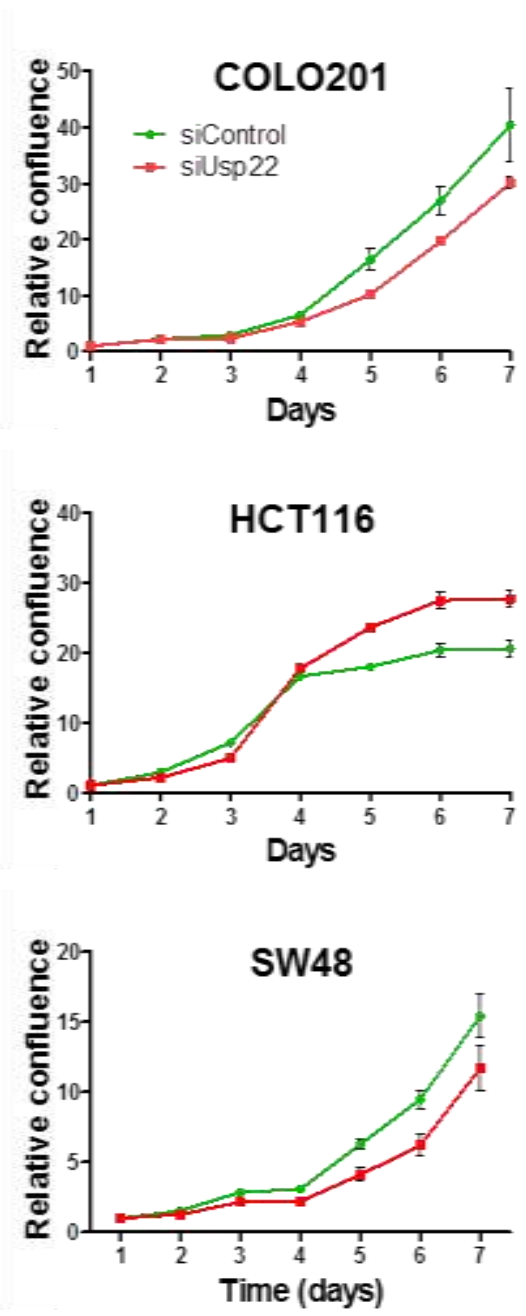
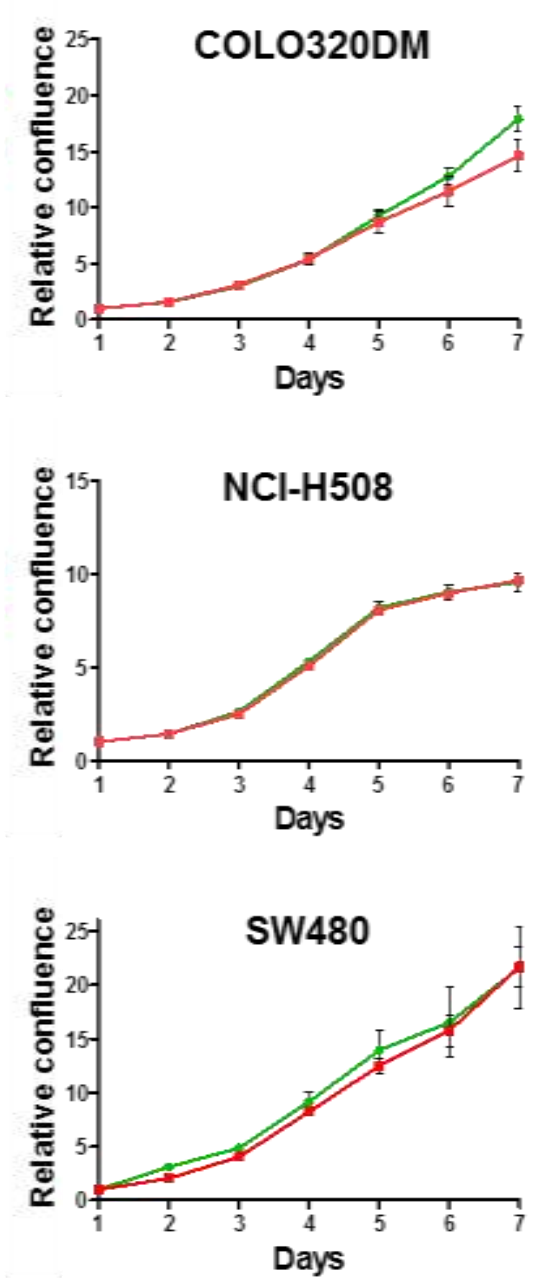
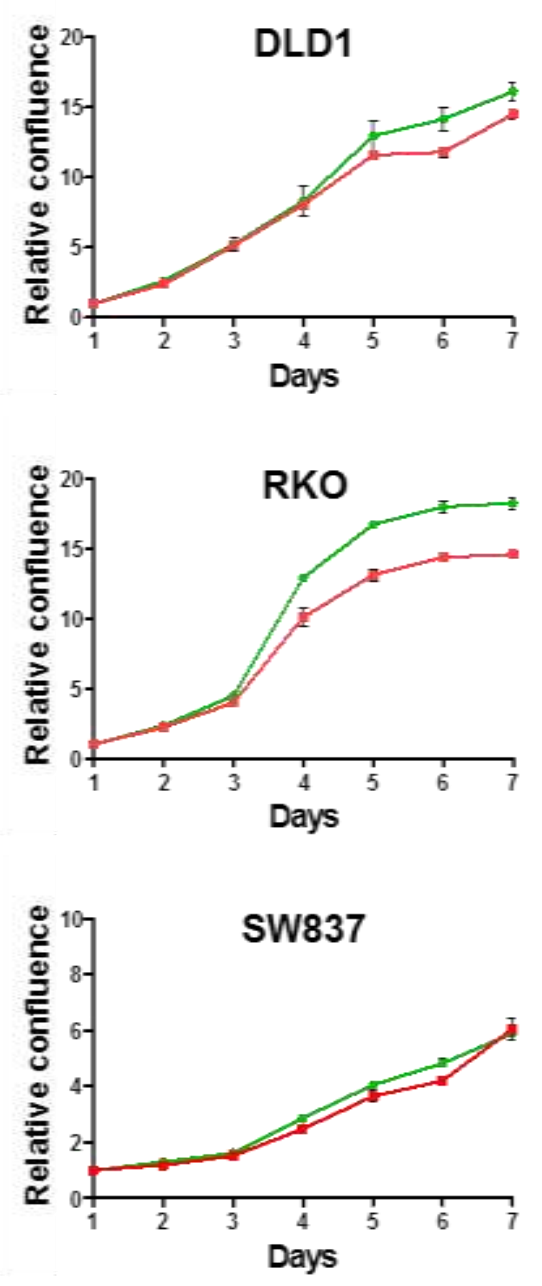

Figure 46: USP22 loss can have differential effects on proliferation of CRC cell lines. Two days after knockdown, the proliferation of nine CRC cell lines was measured every $24 \mathrm{~h}$ using a Celigo ${ }^{\circledR}$ device. The growth rates of some cell lines was not or only marginally affected by USP22 knockdown (NCI-H508, SW480, SW837), while in most cell lines a growth advantage was observed in siControl cells (COLO201, COLO320DM, DLD1, RKO, SW48). HCT116 siUsp22 cells showed increased proliferation. Mean \pm SD, Student's $t$-test, $n=3$. Proliferation effects could neither be correlated with cell line characteristics such as MSI, CIMP and CIN status nor with certain genetic mutations (Table 19). We decided to mainly use HCT116 cells for our future experiments since they resemble the in vivo situation best, i.e. increased growth of colorectal cancer cells upon USP22 loss. 
Table 19: Growth characteristics upon USP22 loss do not correlate with MSI, CIN or CIMP status of CRC cell lines.

\begin{tabular}{|l|c|c|c|c|c|}
\cline { 2 - 6 } & MSI & CIN & CIMP & Usp22 protein levels & $\begin{array}{c}\text { Growth after USP22 } \\
\text { loss }\end{array}$ \\
\hline COL0201 & MSS & + & + & +++ & Down \\
\hline COL0320DM & MSS & + & - & ++++ & Down \\
\hline DLD1 & MSI & - & + & +++ & Down \\
\hline HCT116 & MSI & - & + & ++ & Unaffected \\
\hline NCI-H508 & MSS & + & - & + & Down \\
\hline RK0 & MSI & - & + & ++++ & Down \\
\hline SW48 & MSI & - & + & +++ & Unaffected \\
\hline SW480 & MSS & + & - & +++ & Unaffected \\
\hline SW837 & MSS & + & + & ++ & \\
\hline
\end{tabular}

It was reported that USP22 and its homolog USP27X can have similar functions (Atanassov et al., 2016). To test their effect on CRC cell morphology and proliferation, both peptidases were knocked down. Moreover, when performing siRNA-mediated knockdowns, the specificity of the siRNA should be confirmed. In this case we aimed to verify whether the effects on proliferation are mediated by the loss of USP22 alone or whether the highly homologous USP27X was also targeted by the USP22-siRNA. For this purpose, two cell lines with opposing consequences of USP22 loss were selected, i.e. SW48 in which USP22 knockdown decreased cell growth and HCT116 where proliferation was increased. Cells were transfected with siRNAs targeting USP22 and USP27X for single and double knockdowns. The cell morphology was not affected by silencing USP22, USP27X or both genes simultaneously (Figure 47A). 

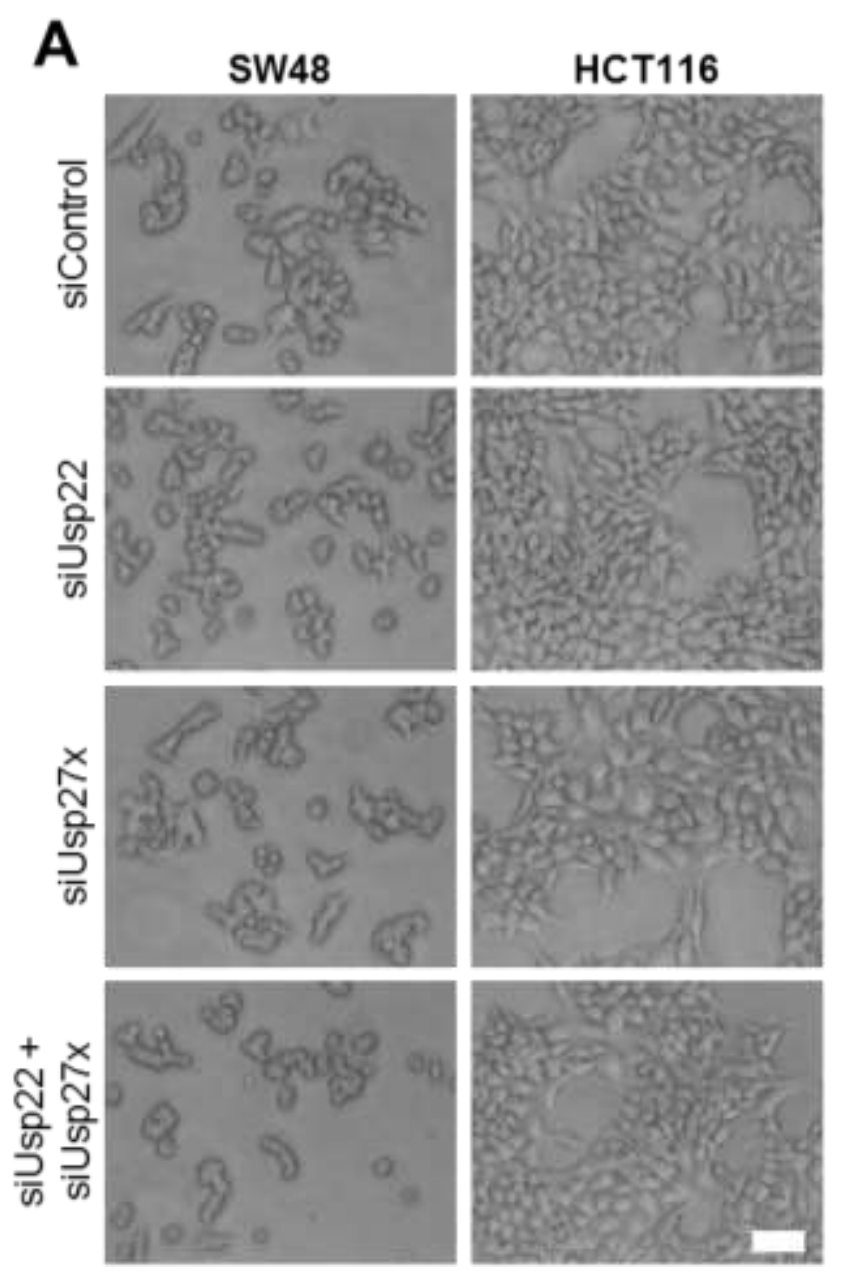

B

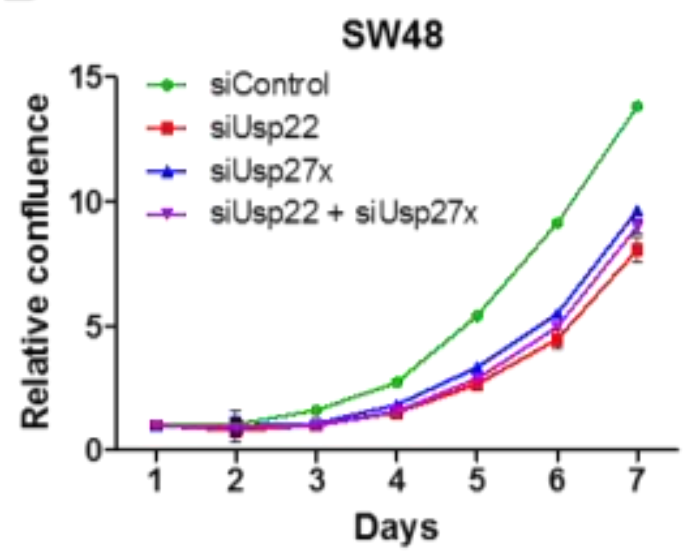

C

HCT116

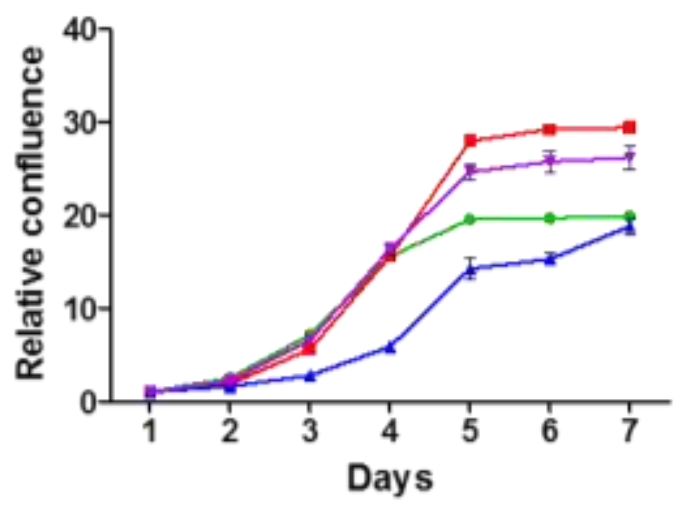

Figure 47: USP22 and USP27X can have opposing effects on CRC cell proliferation. Effects of the loss of USP22, USP27X and both peptidases on morphology and proliferation were evaluated. (A) No morphological effect was observed upon siRNA-mediated knockdown. Scale bar: $500 \mu \mathrm{m}$. (B) While in SW48 cells the loss of USP22 and USP27X had similar consequences, in (C) HCT116 opposing effects of these two peptidases was observed by Celigo ${ }^{\circledR}$ measurement. After the dual knockdown in HCT116 cells, the anti-proliferative effect of USP27X loss was rescued by the additional depletion of USP22. Mean \pm SD, Student's $t$ test, $\mathrm{n}=3$.

Cell proliferation was assessed by Celigo ${ }^{\circledR}$ measurement and as expected, loss of USP22 decreased proliferation levels in SW48 (Figure 47B) and increased them in HCT116 cells (Figure 47C). Notably, also the knockdown of USP27X had opposing effects in the two tested cell lines. SW48 cells grew slightly faster than after loss USP2. In contrast, USP27X reduction significantly decreased proliferation in HCT116 cells. Interestingly, in HCT116 cells the simultaneous knockdown of USP22 and USP27X could rescue the severe deceleration in 
growth after siUSP27X treatment. Thus, the possibility that the growth rates after USP22 knockdown were affected by USP27X-related off-target effects was ruled out. Moreover, despite their high homology, USP22 and USP27X can have opposing effects on the proliferation of CRC cells.

For further characterizations we selected four cell lines which reacted differently to the loss of USP22 expression. In SW480 and SW837 cells we could not observe any effects upon the siRNA-mediated knockdown. In contrast, reduced USP22 levels resulted in a proliferation advantage in HCT116 and disadvantage in SW48 cells. The effects on growth behavior obtained by Celigo $^{\circledR}$ measurement (Figure 46) were confirmed in a crystal violet-based proliferation assay (Figure 48A). We postulated that these proliferative effects were translatable to the migratory properties of the cells upon USP22 loss. Indeed, upon knocking down USP22, HCT116 cells were characterized by increased migration potential while the opposite was observed in SW48 cells (Figure 48B). Interestingly, USP22-depleted HCT116 cells formed more colonies (Figure 48C) and grew better in an anchorage-independent manner (Figure 48D) compared to cells treated with the control siRNA as visualized by crystal violet staining. To translate these findings to the observations we made in our in vivo experiments in which the APC protein was mutated in some mouse cohorts, we aimed to observe the Wnt signaling pathway activity in CRC cells. In addition, increased Wnt activity is a common occurrence in human CRCs. For this purpose we have performed a luciferase assay. Briefly, reporter plasmids containing a luciferase reporter gene under the control of TCF/LEF response elements were introduced into cells. Once, the canonical Wnt signaling is active and luciferase substrates are added, fluorescent signal can be measured. A knockdown of $\beta$-catenin (si $\beta$ catenin) was used as a negative control. Characterization of HCT116 cells revealed slightly decreased Wnt signaling activity after the loss of USP22 (Figure 48E). In SW480 cells which were used as a control, the same outcome was obtained. In summary, HCT116 cells were characterized by increased proliferation and migration rates upon USP22 ablation. Moreover, 
they were able to grow better in anchorage-independent manner. Using a luciferase assay, we demonstrated that these properties were not driven by increased Wnt pathway activity.
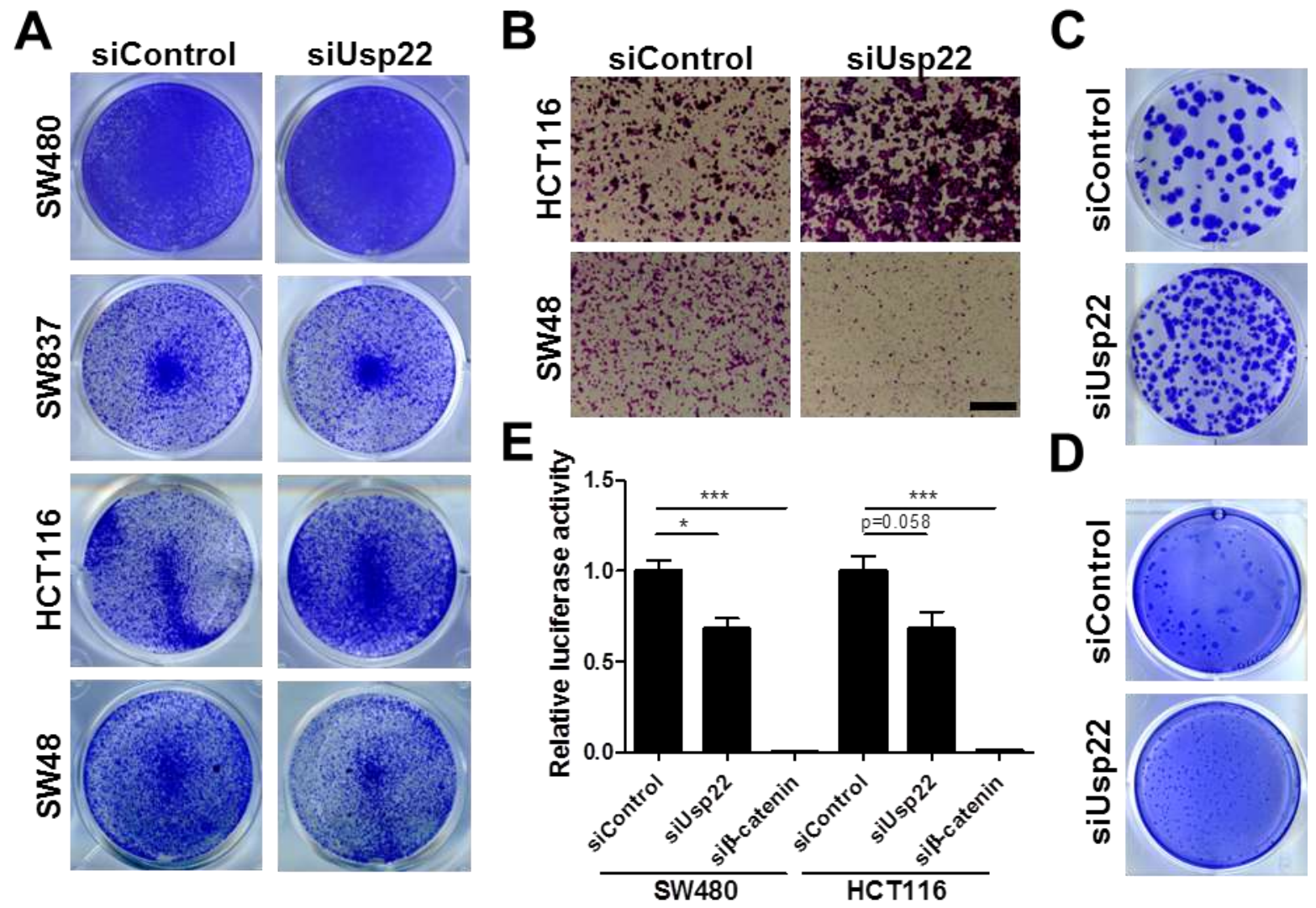

Figure 48: USP22 is involved in regulation of migration potential, anchorageindependent growth and Wnt signaling pathway activity. (A) As observed in the Celigo ${ }^{\circledR}$ measurements, USP22 depletion can have differential effects on proliferation as shown by this crystal violet-based proliferation assay $48 \mathrm{~h}$ after knockdown. (B) $24 \mathrm{~h}$ after siRNA transfection, cells were seeded into trans-well migration inserts. After $48 \mathrm{~h}$ cells were stained with crystal violet. USP22 loss resulted in increased migration potential in HCT116 cells while the opposite was observed in SW48 cells. Scale bar: $500 \mu \mathrm{m}$ (C) Knockdown enhanced colony formation after 4-5 days (D) and cell growth in anchorage-independent manner after 2 weeks. (E) A luciferase reporter assay demonstrated decreased Wnt pathway activity in siUsp22 cells, in both HCT116 and SW480 cells. Mean \pm SD, Student's $t$-test, $n=3$. 


\subsection{USP22 regulates proliferation and differentiation processes in CRC cells}

To determine the effect of USP22 loss on global gene expression and to potentially identify the mechanism underlying the previous results, we pursued our investigations with transcriptomewide gene expression analyses using mRNA-sequencing. For this purpose, we selected SW837 and SW480 cells and treated them with control- and USP22-siRNA. We decided to use SW480 and SW837 for this approach since their gross phenotype was not or only minimally affected by USP22 loss and therefore of secondary effects, e.g. due to dramatic changes in cell phenotype, were expected to be low. After RNA isolation and quality control using RNA gel electrophoresis, libraries were generated and sequencing reactions were performed at the TAL.

Per cell line and condition (siControl and siUsp22) we have analyzed four replicates. The sample variance and data quality was assessed by hierarchical clustering and principal component analysis. There was a clustering of replicates verifying minimal deviations between these samples (Figure 49A). As expected, the difference between the two cell lines was immense. In addition, a variation between siControl and siUsp22 was detected as revealed by PCA plot (Figure 49B). However, the loss of USP22 seemed to have a higher impact on gene expression in SW837 than in SW480 cells. In addition, we compared the replicate variance in genes with high fold changes (log2fold change value \pm 1 , padj $\leq 0.05$ ). As visualized in these heatmaps using the Morpheus tool, replicates show a high similarity (Figure 49C). 


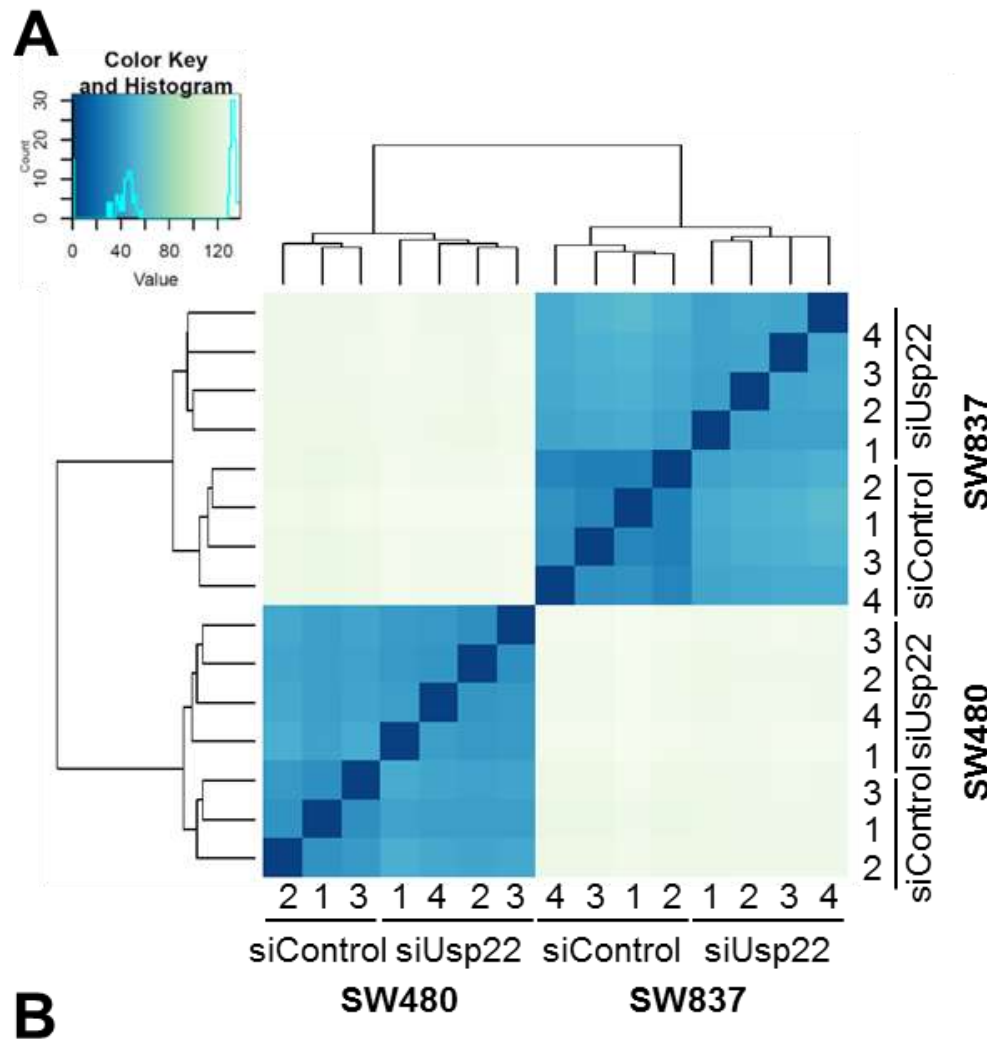

C

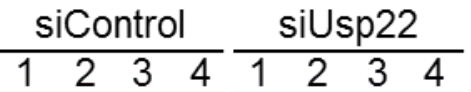

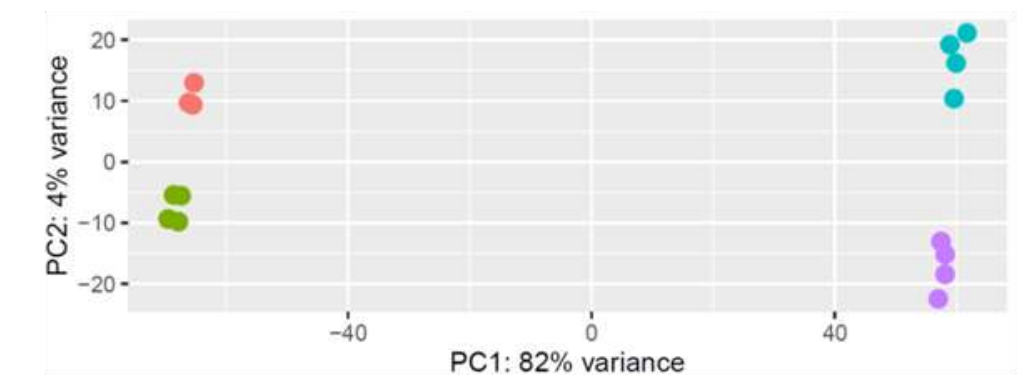

SW837: siControl siUsp22 sW480: siControl $\bullet$ siUsp22
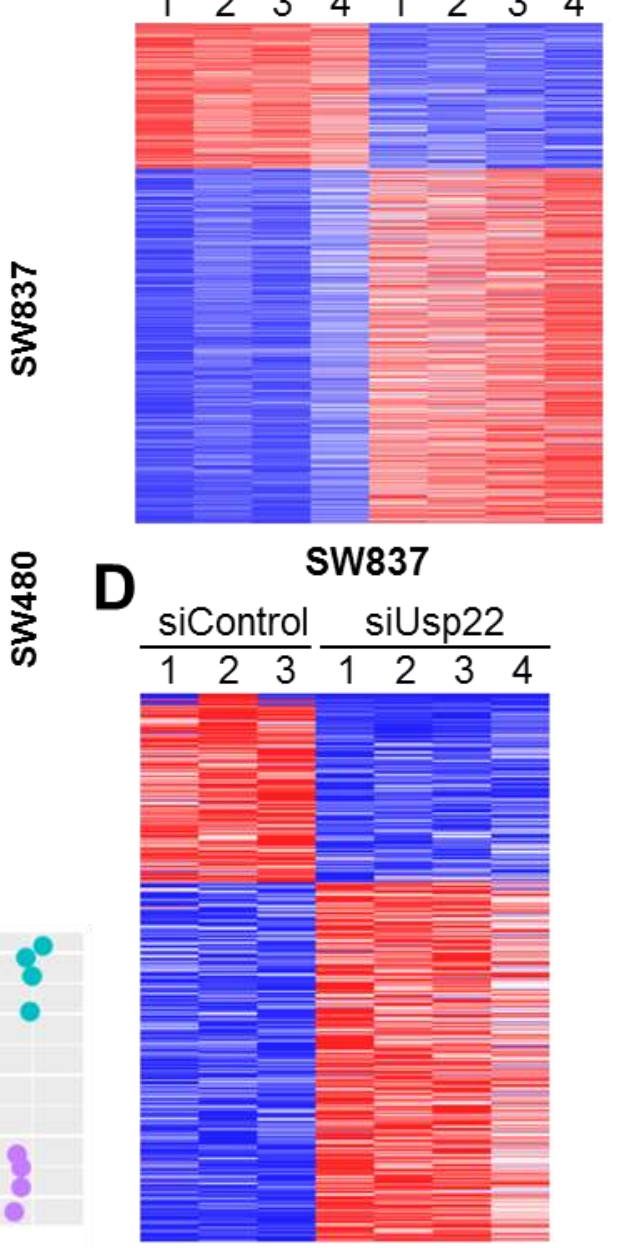

\section{SW480}

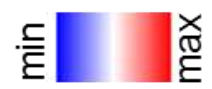

Figure 49: Quality control of SW480 and SW837 replicates used for mRNA-seq analysis. (A) Hierarchical clustering and (B) PCA plot indicate a high similarity between replicates $(n=4)$. There is a significant difference between the two cell lines, SW480 and SW837. (C) Highly regulated genes (log2fold change value \pm 1 , padj $\leq 0.05$ ) and their expression in all replicates are shown in SW837 and SW480 cells using the Morpheus tool. Variations among the replicates are marginal. The color code indicates the relative minimum ( $\mathrm{min}$ ) and maximum (max) expression value per gene. 
Initially, we decided to examine genes which are differentially expressed in SW837, SW480 cells and MEFs (see section 3.1). Since these cells are highly different from each other, we expected that we would find only few overlapping genes but that these would be of high significance since they were affected by USP22 loss in different biological systems. For this purpose, genes with a log2 fold change of at least 0.8 in all three cell types were selected. These genes were divided into down- (Figure 50A) and upregulated (Figure 50B) genes. Outcomes obtained from SW837 cells were shown in the left, from SW480 in the middle and from MEFs in the right column. Moreover, the heatmaps were subdivided into four segments in which genes strongly regulated in all three cell types (segment on the top), in SW837 (second segment), SW480 cells (third segment) and MEFs (bottom) were depicted. Each line represents one gene and expression changes can be compared among SW837, SW480 cells and MEFs by comparing the three columns. Genes which were not expressed in all cell types were excluded from the analyses. While SW837 and SW480 showed similar tendencies, the results obtained from MEFs were highly heterogeneous. Approximately half of the genes upregulated in the human cell lines were also upregulated in MEFs. In the downregulated genes, the overlap between human and murine samples was on average $60 \%$. When focusing on genes differentially regulated in Usp22 laczlacz MEFs, there is only low accordance with the human cells. In addition, many genes which were differentially regulated in the human cell lines were not detected in MEFs and were therefore excluded from the analyses. Due to the low accordance between human and murine samples, we decided to no longer take the MEFs into account but instead focus on the human cell lines. 


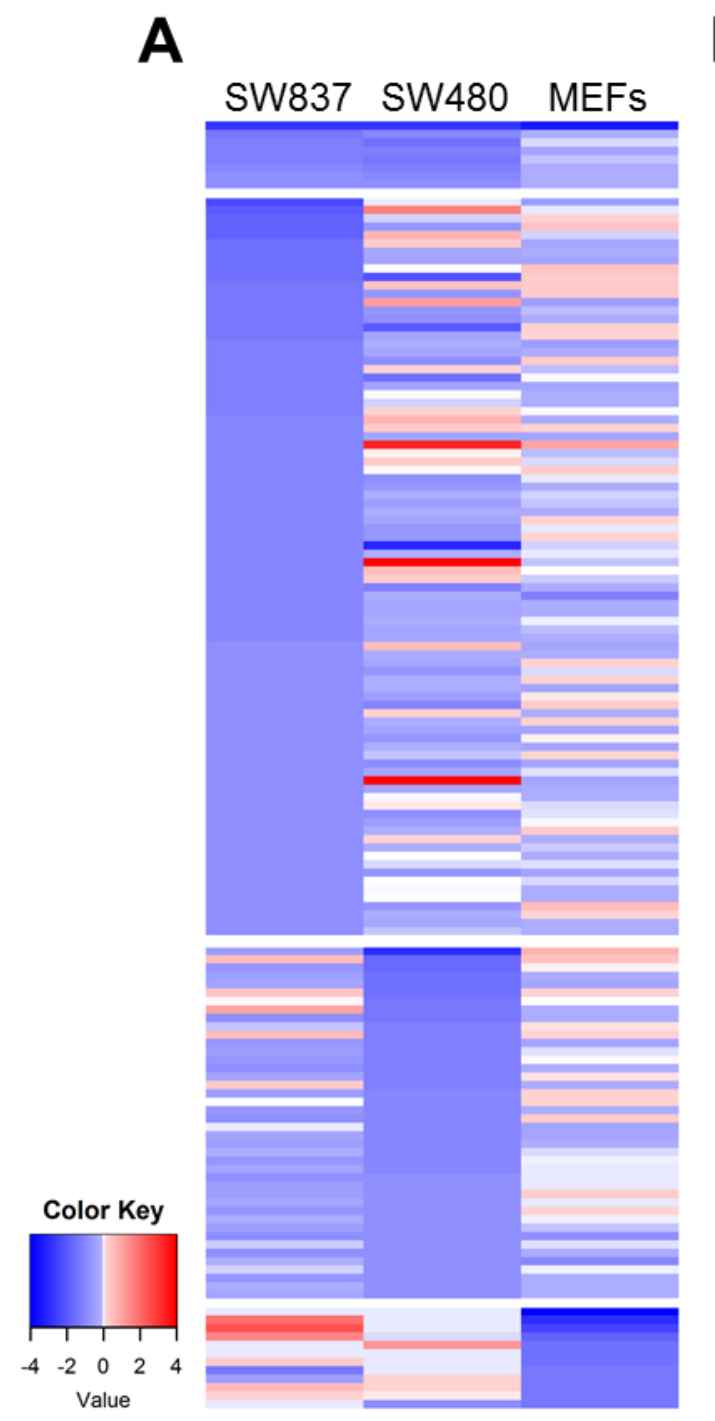

\section{B}

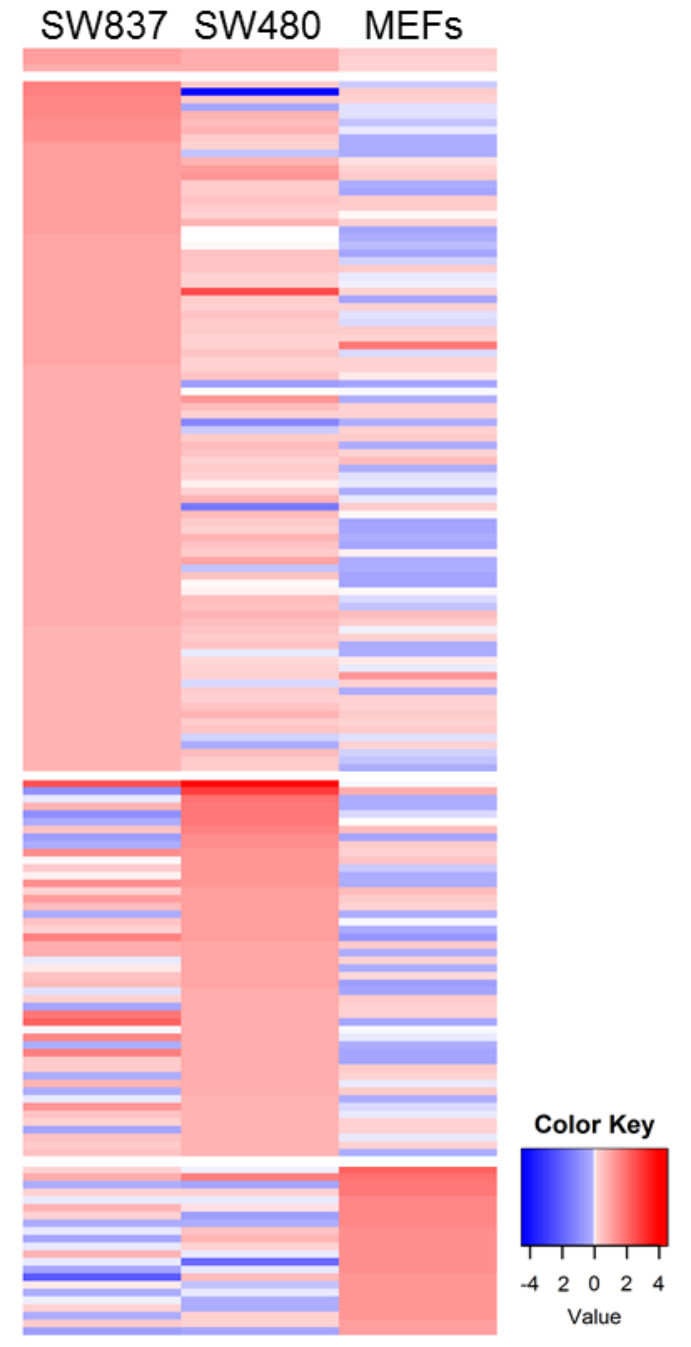

Figure 50: Moderate consistency of differentially expressed genes after USP22 depletion in SW480, SW837 cells and MEFs. mRNA-seq and microarray outcomes were compared by displaying differentially regulated genes (log2fold change value \pm 0.8 , padj $\leq 0.05$ ) in SW837, SW480 and MEFs in a heatmap. Highly (A) down- or (B) upregulated genes of each cell type were indicated with the corresponding expression values of the other conditions. The four segments indicate genes strongly regulated in all three cell types (top segment), in SW837 (second segment), SW480 cells (third segment) and MEFs (bottom). Due to low agreement with human CRC cells, MEFs were excluded from subsequent analyses.

To obtain an overview on the genes differentially regulated upon USP22 knockdown in SW837 and SW480 cells, new heat maps were generated. Similar to the previous heatmap, genes with a $\log 2$ fold change of at least 0.8 in both cell lines were depicted and divided into down- (Figure 51A) and upregulated (Figure 51B) genes. Results obtained from SW837 cells were visualized 
in the left column while outcomes from SW480 were shown in the right column. The three segments indicate genes differentially regulated in both cell lines (segment on the top), in SW837 (middle) and SW480 cells (bottom). Despite the heterogeneity expected when comparing two cell lines, several differentially regulated genes could be identified showing the same tendency in both, SW480 and SW837.



Figure 51: Comparison of differentially expressed genes after USP22 depletion in SW837 and SW480 cells. Significantly (A) down- or (B) upregulated genes (log2fold change value \pm 0.8 , padj $\leq 0.05$ ) revealed by mRNA-seq using SW837 and SW480 cells have been depicted in a heatmap. Highly affected genes per cell line are shown with the corresponding expression values (color key). The three segments indicate genes differentially regulated in both cell lines (first segment), in SW837 (second segment), SW480 cells (third segment). 
By means of the Gene Ontology visualization tool REViGO (Supek et al., 2011), biological and cellular processes associated with USP22 loss in SW837 and SW480 cells were depicted. Previously, using Usp22 lacz/lacz MEFs, we detected the enrichment of several proliferation and development-related pathways. Indeed, GO analysis of SW837 (Figure 52A, Table 20) and SW480 cells (Figure 52B, Table 21) revealed similar outcomes. Mainly, terms associated with development and proliferation, as well as cell communication were enriched in both cell types. In addition, GO terms revealed that USP22 is associated with the regulation of signaling processes and cell communication which could be associated with extracellular stimuli. Together, analysis of our mRNA-seq analysis revealed that there is only a low agreement between MEFs we have analyzed earlier and CRC cells. The expression of numerous genes was affected by USP22 depletion and besides an involvement in proliferation- and differentiation-related processes, GO analysis indicated a potential role of USP22 in the response to extracellular stimuli. 

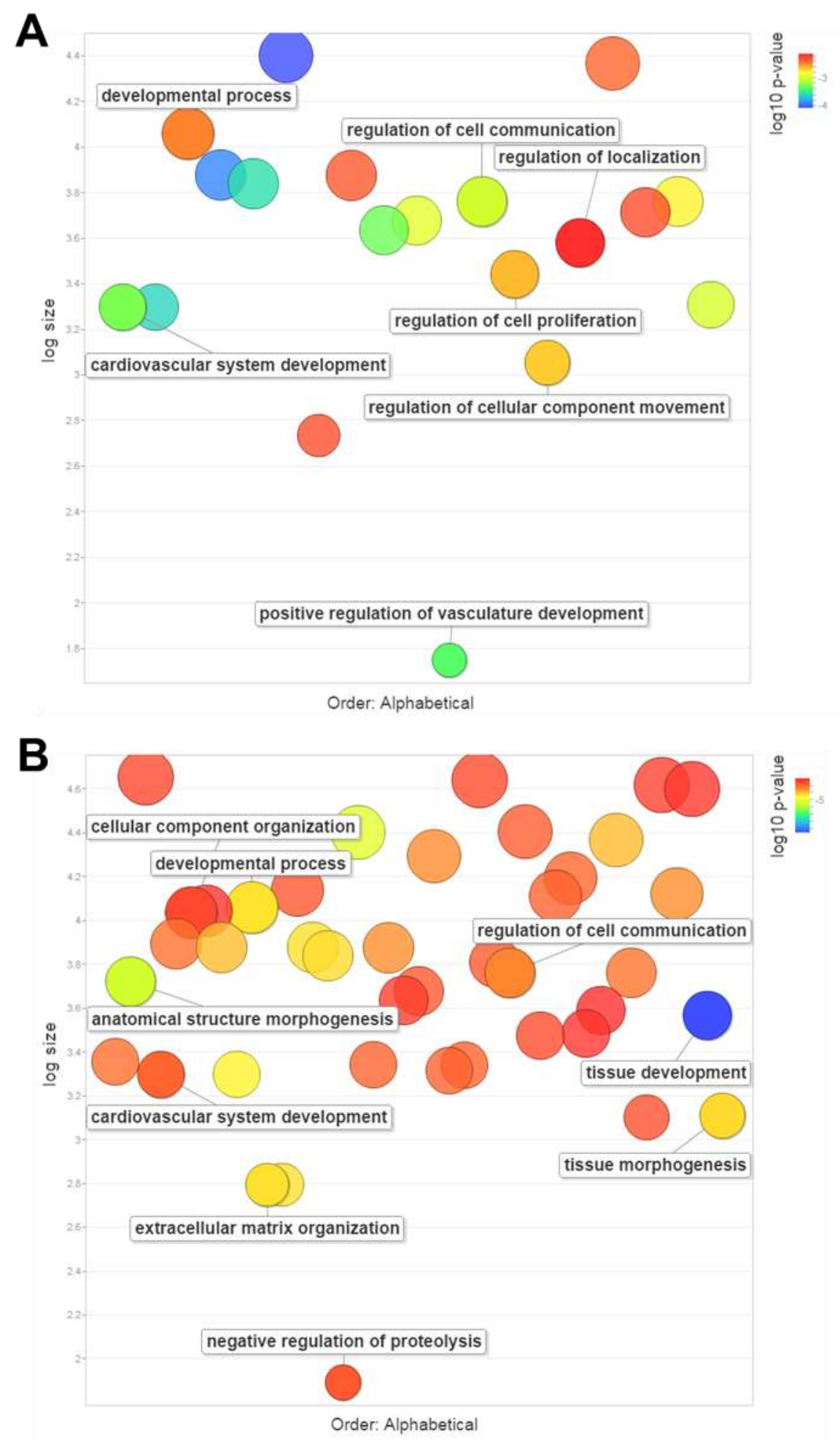

Figure 52: Gene Ontology terms enriched after USP22 loss in SW837 and SW480 cells. REViGO analysis revealed enriched cellular processes upon USP22 knockdown in (A) SW837 and (B) SW480 cells. In both cell lines mainly GO terms associated with development, proliferation and cell communication were affected. 
Table 20: GO terms enriched in siUsp22 SW837 cells as revealed by GOTermFinder.

\begin{tabular}{|c|c|c|c|c|}
\hline Term ID & Gene Ontology term & $\begin{array}{l}\text { Cluster } \\
\text { frequency }\end{array}$ & $\begin{array}{l}\text { Corrected } \\
p \text {-value }\end{array}$ & FDR \\
\hline GO:0032502 & Developmental process & $24.3 \%$ & 0.00336 & 0 \\
\hline GO:0048583 & Regulation of response to stimulus & $18.1 \%$ & 0.00432 & 0 \\
\hline GO:0010646 & Regulation of cell communication & $16.0 \%$ & 0.00098 & 0 \\
\hline GO:0023051 & Regulation of signaling & $16.0 \%$ & 0.00174 & 0 \\
\hline GO:0009893 & Positive regulation of metabolic process & $14.9 \%$ & 0.00121 & 0 \\
\hline GO:0009966 & Regulation of signal transduction & $14.2 \%$ & 0.0054 & 0 \\
\hline GO:0032879 & Regulation of localization & $12.8 \%$ & 0.00736 & 0 \\
\hline GO:0042127 & Regulation of cell proliferation & $9.7 \%$ & 0.00243 & 0 \\
\hline GO:0072359 & Circulatory system development & $8.0 \%$ & 0.00025 & 0 \\
\hline GO:0009725 & Response to hormone & $7.3 \%$ & 0.00104 & 0 \\
\hline GO:0051270 & $\begin{array}{l}\text { Regulation of cellular component } \\
\text { movement }\end{array}$ & $6.6 \%$ & 0.00219 & 0 \\
\hline GO:0072358 & Cardiovascular system development & $6.2 \%$ & 0.00055 & 0 \\
\hline GO:0001568 & Blood vessel development & $5.9 \%$ & 0.00142 & 0 \\
\hline GO:0001944 & Vasculature development & $5.9 \%$ & 0.00223 & 0 \\
\hline GO:0045765 & Regulation of angiogenesis & $3.5 \%$ & 0.00731 & 0 \\
\hline
\end{tabular}


Table 21: GO terms enriched in siUsp22 SW480 cells as revealed by GOTermFinder.

\begin{tabular}{|c|c|c|c|c|}
\hline Term ID & Gene Ontology term & $\begin{array}{l}\text { Cluster } \\
\text { frequency }\end{array}$ & $\begin{array}{l}\text { Corrected } \\
p \text {-value }\end{array}$ & FDR \\
\hline GO:0019222 & Regulation of metabolic process & $35.4 \%$ & 0.00121 & 0 \\
\hline GO:0016043 & Cellular component organization & $31.7 \%$ & 0.00381 & 0 \\
\hline GO:0032502 & Developmental process & $30.5 \%$ & 0.0000551 & 0 \\
\hline GO:0030154 & Cell differentiation & $20.7 \%$ & 0.0009 & 0 \\
\hline GO:0048513 & Animal organ development & $20.1 \%$ & 0.0000467 & 0 \\
\hline GO:0009653 & Anatomical structure morphogenesis & $19.5 \%$ & 0.00000422 & 0 \\
\hline GO:0010646 & Regulation of cell communication & $19.5 \%$ & 0.00046 & 0 \\
\hline GO:0023051 & Regulation of signaling & $19.5 \%$ & 0.00073 & 0 \\
\hline GO:0009888 & Tissue development & $18.9 \%$ & $3.04 \mathrm{E}-10$ & 0 \\
\hline GO:0010647 & Positive regulation of cell communication & $12.2 \%$ & 0.0014 & 0 \\
\hline GO:0048729 & Tissue morphogenesis & $8.5 \%$ & 0.0000702 & 0 \\
\hline GO:0001568 & Blood vessel development & $7.9 \%$ & 0.00083 & 0 \\
\hline GO:0072358 & Cardiovascular system development & $7.9 \%$ & 0.00135 & 0 \\
\hline GO:0030198 & Extracellular matrix organization & $6.7 \%$ & 0.0000584 & 0 \\
\hline GO:0045861 & Negative regulation of proteolysis & $6.1 \%$ & 0.00212 & 0 \\
\hline
\end{tabular}




\subsection{USP22 regulates the stability of heat shock proteins}

GO analysis revealed that USP22-regulated genes are involved in the response to stimuli. As reviewed by Shim and Karin, mRNA expression can be affected by stimuli such as developmental, nutritional and pharmacological alterations, however, also by environmental factors such as temperature changes (Shim and Karin, 2002). Interestingly, mRNA-seq data indicated that levels of heat shock proteins were decreased by reduced USP22 expression in both, SW837 and SW480 cells. Generally, there are two HSP90 isoforms present in the cytoplasm: the stress-inducible HSP90a and the constitutively expressed HSP90ß (Mimnaugh et al., 1996; Whitesell and Lindquist, 2005). Notably, our mRNA-seq results only indicated altered expression of constitutively active family members.

To verify this outcome we performed siRNA-mediated knockdown of USP22 in SW837, SW480, HCT116 and SW48 cells and determined Heat Shock Protein 90 Alpha Family Class B Member 1 (HSP9OAB1) mRNA levels. Indeed, we confirmed in all four cell lines that HSP9OAB1 expression levels were decreased by approximately $50 \%$ in siUsp22 cells (Figure 53A). This phenomenon was observed on protein level as well as shown for HCT116 cells (Figure 53B). Since heat shock proteins play a crucial role in the cells' adaptation to environmental changes, we speculated that reduced HSP90AB1 levels can sensitize siUsp22 cells to high temperatures. For this purpose, we subjected HCT116 cells to heat shocks $48 \mathrm{~h}$ after USP22 knockdown. As incubation at $42^{\circ} \mathrm{C}$ only yielded minimal differences (data not shown), cells were incubated at $50^{\circ} \mathrm{C}$ for $1 \mathrm{~h}$. As expected, siUsp22 $\mathrm{HCT} 116$ control cells grown at $37^{\circ} \mathrm{C}$ grew faster than the wild types (Figure $53 \mathrm{C}$ ). Comparing this growth rate to the cell number after incubation at $50^{\circ} \mathrm{C}$, the cell number was reduced by $17 \%$ in siControl and by $24 \%$ in siUsp22 cells. In summary, we could not only detect a decrease of HSP90AB1 on mRNA and protein level in siUsp22 cells, but also observed high temperature sensitivity in these cells. 
A
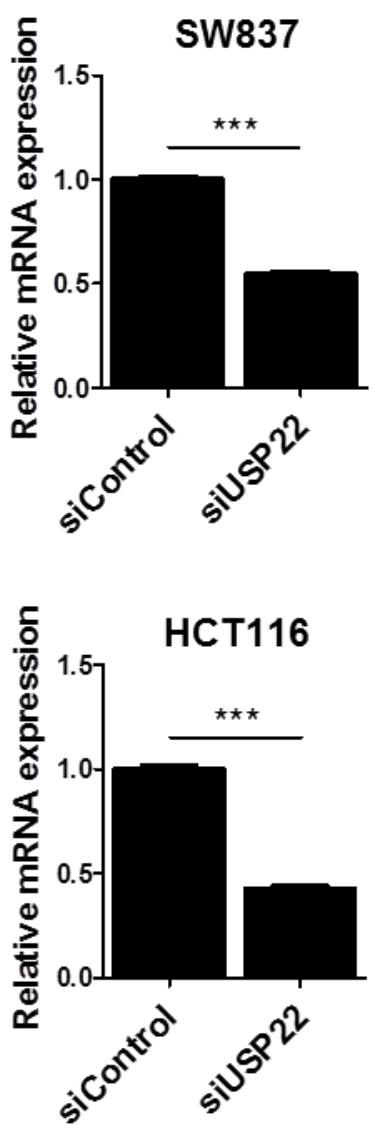
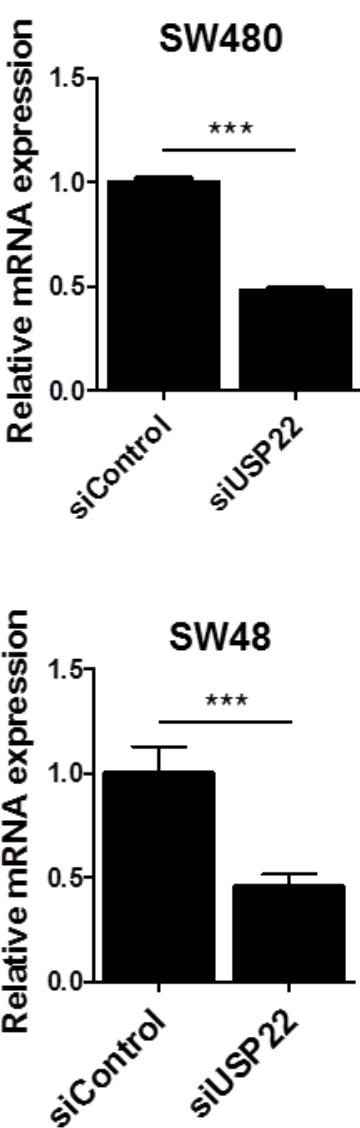

B
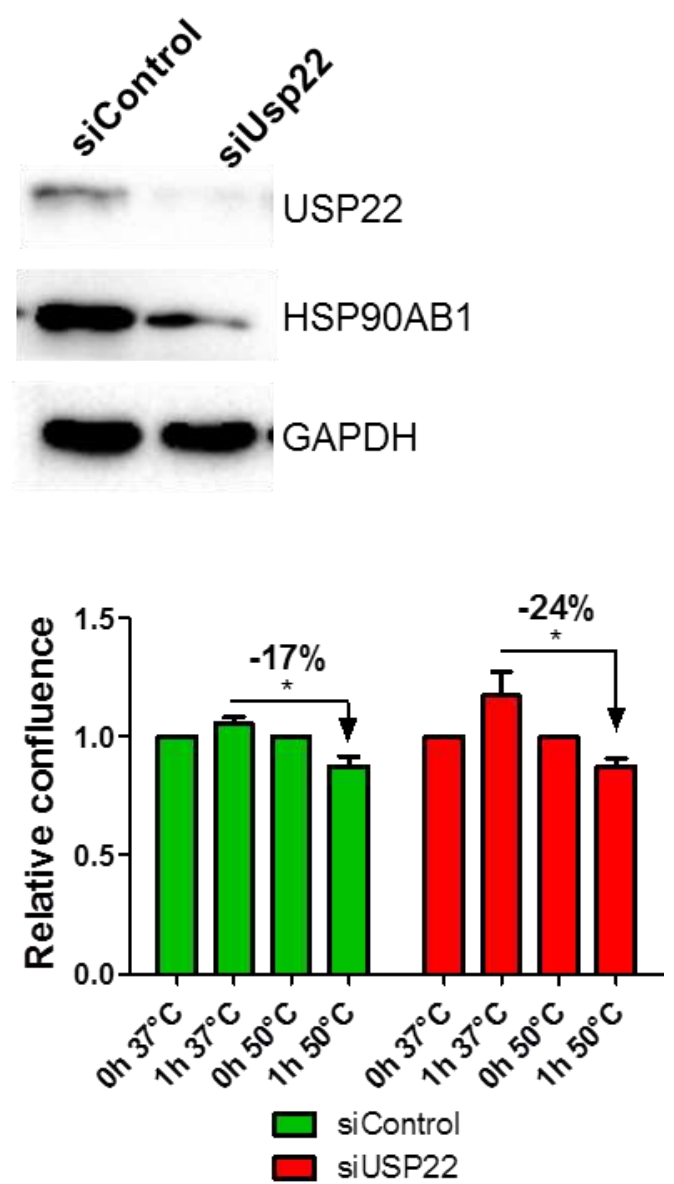

Figure 53: The expression of the heat shock protein HSP90AB1 is reduced following USP22 depletion leading to increased cell sensitivity. (A) A reduction of HSP9OAB1 mRNA levels was detected by qRT-PCR upon USP22 knockdown in four cell lines (SW837, SW480, HCT116, SW48). Mean \pm SD, Student's $t$-test, $n=3$. (B) In HCT116 cells this decrease was confirmed on protein level. (C) $48 \mathrm{~h}$ after siRNA transfection, HCT116 USP22 wild type and knockdown cells were incubated either at $37^{\circ} \mathrm{C}$ or $50^{\circ} \mathrm{C}$ for $1 \mathrm{~h}$. Compared to the normal growth rates at $37^{\circ} \mathrm{C}$, incubation at $50^{\circ} \mathrm{C}$ decreased cell numbers in siControl cells by $17 \%$ and in siUsp22 cells by $24 \%$. Mean \pm SD, Student's $t$-test, $n=4$.

To obtain insights into the mechanisms responsible for USP22-mediated regulation of HSP90 levels, we decided to investigate the functional interaction between USP22 and HSP90AB1 by Co-IP. For this purpose, we conjugated protein G sepharose (PGS) beads with antibodies targeting either USP22 or HSP90. These beads have been incubated with HCT116 protein lysate to precipitate potential USP22-HSP90AB1 protein complexes. As expected, in the control conditions (PGS and IgG) neither USP22 nor HSP90AB1 were present while all IgG- 
bands were visible, confirming absence of unspecific USP22- and HSP90-binding to the beads or IgG. By using antibodies directed against either USP22 or HSP90, we were able to precipitate USP22-HSP90AB1 complexes (Figure 54A) implying direct interaction of these two proteins. Furthermore, we investigated whether the ability of USP22 to deubiquitinate proteins is associated with the regulation of HSP90 levels. We aimed to determine whether the reduction of HSP90AB1 is due to ubiquitination and whether this loss can be prevented by inhibiting the proteasome. Moreover, we tested whether this phenomenon is USP22dependent. For this purpose, we have added the proteasome inhibitor MG-132 to siControl and siUsp22 cells. As expected, loss of USP22 resulted in decreased HSP90AB1 levels (Figure 54B). However, when the proteasome inhibitor was added, HSP90AB1 amounts remained nearly unchanged. This outcome suggested that the reduced HSP90AB1 levels were caused by proteasomal degradation due to ubiquitination.
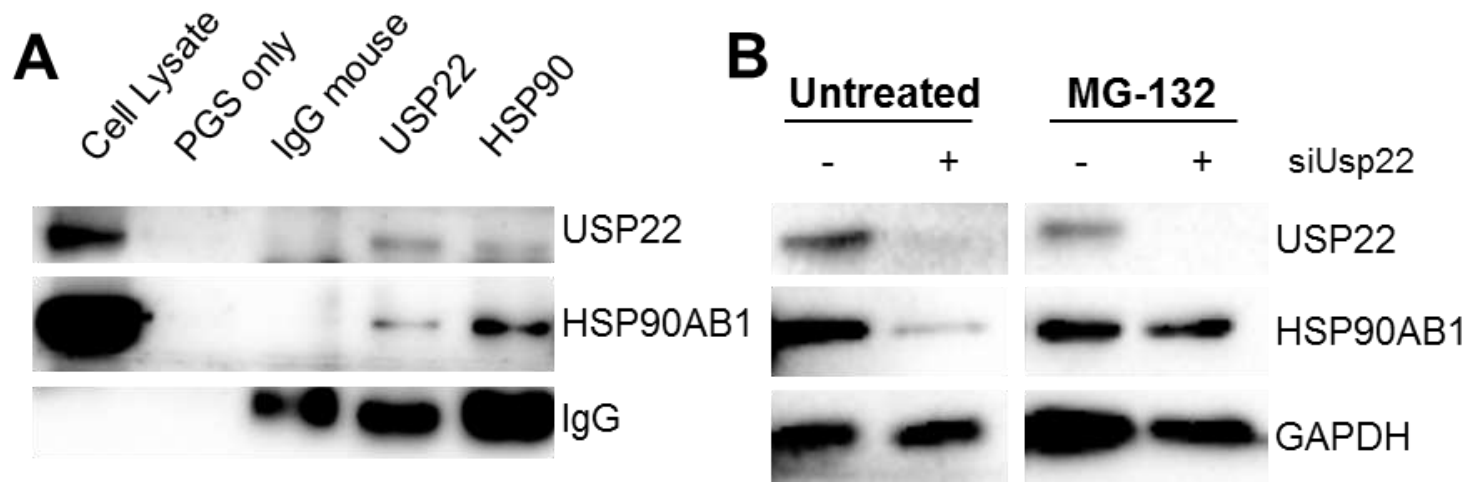

Figure 54: HSP90AB1 interacts with USP22 and is targeted by proteasomal degradation in the absence of USP22. (A) Co-IP was performed to investigate the direct interaction between USP22 and HSP90AB1. USP22-HSP90AB1 complexes were precipitated with both, anti-USP22- and anti-HSP90AB1-conjugated beads. (B) Under normal conditions HSP90 levels decreased in siUsp22 cells. When the proteasome is inhibited by MG-132 this degradation was prevented. 


\subsection{Induction of synthetic lethality in USP22-deficient CRC cells}

Based on these findings we assumed that the residual HSP9O levels are essential for the survival and stress-resistance of siUsp22 cells. We therefore hypothesized that diminishing HSP90 completely would result in decreased cell survival. Moreover, since targeting USP22 directly could have tumor-promoting outcomes, our proposed strategy would reflect an attractive targeting approach based on the concept of synthetic lethality. Thus, HCT116 cells were treated with the HSP90 inhibitor Ganetespib under wild type and USP22 knockdown conditions $48 \mathrm{~h}$ after siRNA transfection. To determine the general sensitivity towards this inhibitor, cells were treated with increasing Ganetespib concentrations for $48 \mathrm{~h}$.
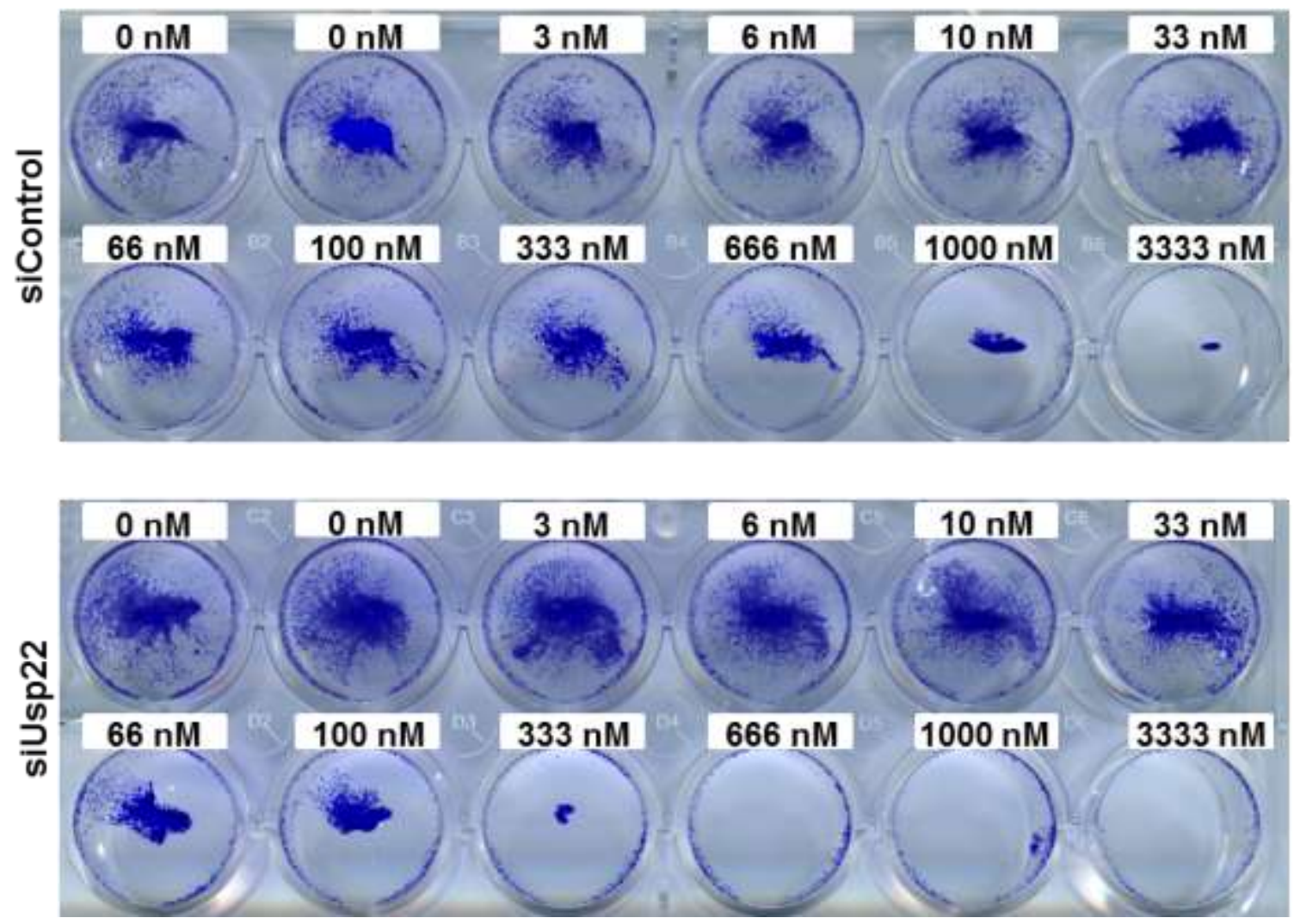

Figure 55: USP22-depleted cells are more sensitive to the HSP90 inhibitor Ganetespib. $48 \mathrm{~h}$ after siRNA transfection, siControl and siUsp22 HCT116 cells were treated with increasing concentrations of the HSP90 inhibitor Ganetespib for $48 \mathrm{~h}$. Surviving cells were visualized by crystal violet staining. USP22-depleted cells showed increased sensitivity towards this drug. 
Subsequently, surviving cells were stained with crystal violet. Indeed, we observed that USP22depleted cells were more sensitive towards this inhibitor (Figure 55). While only few siUsp22 cells could withstand treatment with $333 \mathrm{nM}$ Ganetespib, siControl cells could tolerate 10-fold higher concentrations.

Consequently, we aimed to confirm our findings by testing for apoptosis markers on protein levels upon USP22 loss and/or HSP90 inhibition. USP22 wild type and-depleted cells were treated with $100 \mathrm{nM}$ Ganetespib or DMSO for 48 h. Protein lysates were subjected to western blot analysis. In conformity with our hypothesis, in siUsp22 cells treated with Ganetespib increased levels of the apoptosis markers cleaved PARP and BIM were observed (Figure 56). In summary, these findings strongly support the potential of USP22-deficient cells to be targeted by HSP90 inhibitors based on the concept of synthetic lethality.
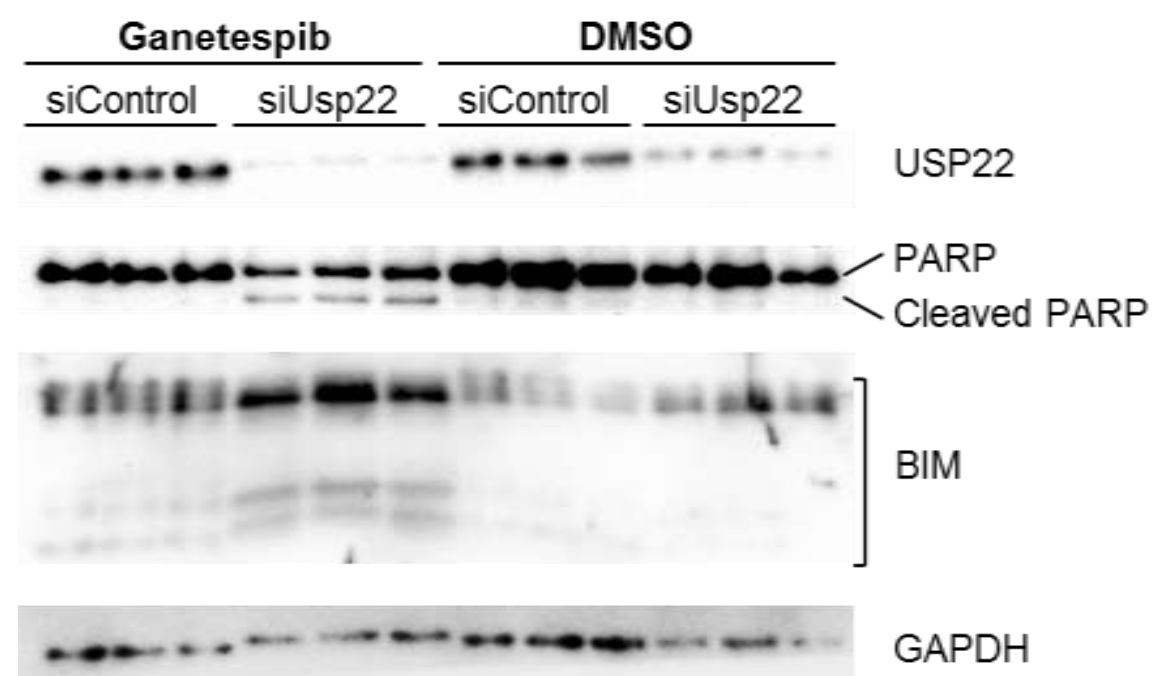

Figure 56: Ganetespib treatment increases apoptosis levels in USP22-depleted cells. 48 h after knockdown, HCT116 cells (siControl and siUsp22) have been treated either with DMSO or $100 \mathrm{nM}$ Ganetespib for $48 \mathrm{~h}$. Protein lysates were evaluated by western blot in triplicates. USP22-depleted cells show an increased abundance of the apoptosis markers cleaved PARP (lower PARP band) and BIM upon treatment with the HSP90 inhibitor Ganetespib.

We further assumed that HSP90 inhibition interferes with its function in facilitating P-TEFb complex formation which therefore cannot be recruited by BRD4 to phosphorylate the RNA Pol 
II. To target another player in this process we treated cells with the BET inhibitor JQ1 which targets among others BRD4. Similar to the previous approach, $48 \mathrm{~h}$ after siRNA transfection, we treated siControl and siUsp22 HCT116 cells with increasing concentrations of the inhibitor for $48 \mathrm{~h}$. Indeed, control cells could tolerate $250 \mathrm{nM}$ JQ1 while most of the USP22-depleted cells could maximally survive a dose of $100 \mathrm{nM}$ (Figure 57A).
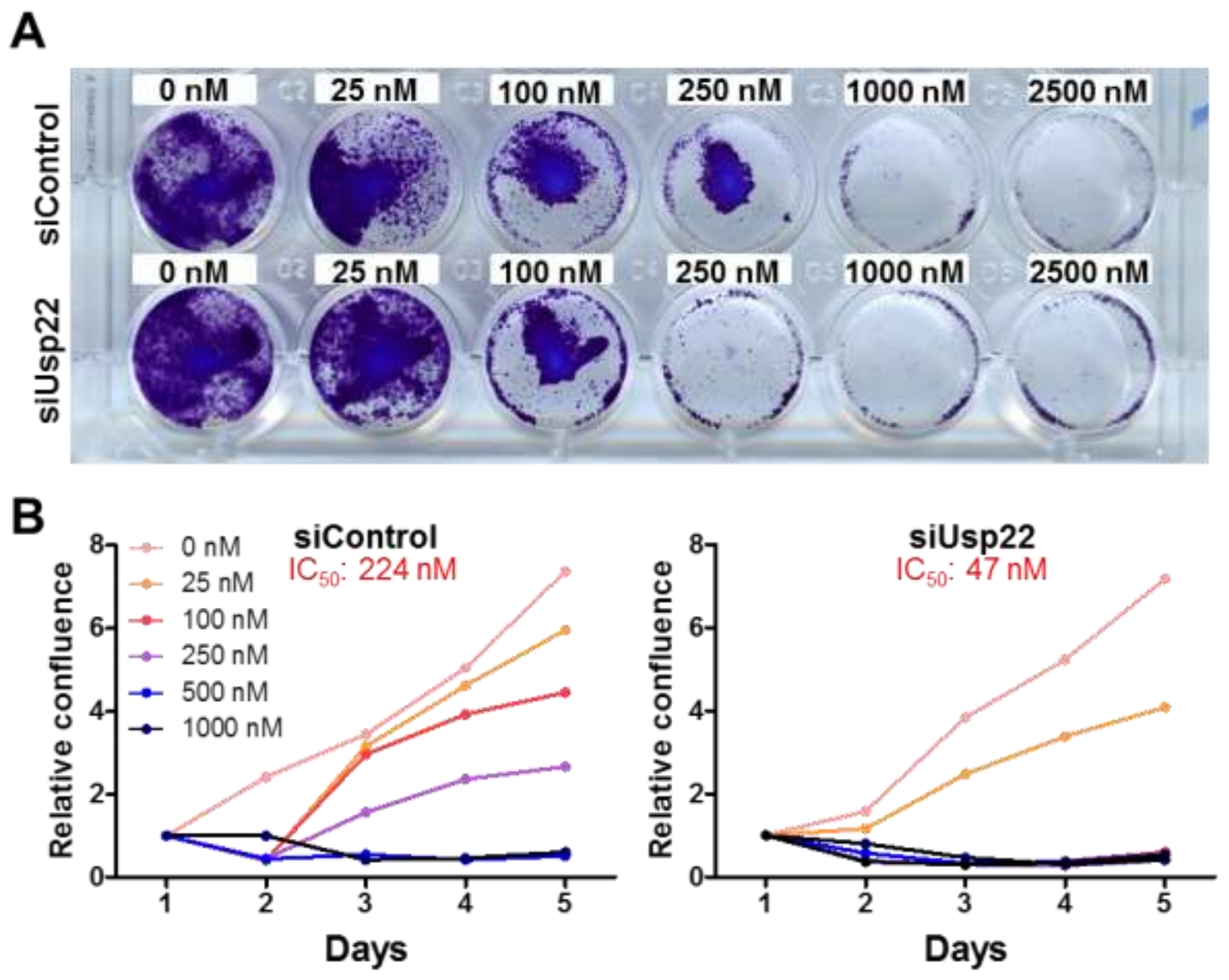

Figure 57: Loss of USP22 elevates sensitivity of CRC cells towards JQ1. $48 \mathrm{~h}$ after HCT116 cells were transfected with control or anti-USP22 siRNAs, cells were treated with increasing concentrations of JQ1 for $48 \mathrm{~h}$. (A) A crystal violet-based assay revealed increased sensitivity towards JQ1 upon knockdown of USP22. (B) Using the Celigo ${ }^{\circledR}$ device the $\mathrm{IC}_{50}$ was determined in these cells. USP22-depleted cells were characterized by a lower $\mathrm{IC}_{50}(47 \mathrm{nM})$ than the controls (224 nM).

To obtain further insights into the sensitivity of siUsp22 cells towards JQ1 treatment, we calculated the $\mathrm{IC}_{50}$ based on Celigo ${ }^{\circledR}$ proliferation measurements. This experiment revealed 
that the $\mathrm{IC}_{50}$ of cells with USP22 wild type levels was $224 \mathrm{nM}$ and of USP22-depleted cells only $47 \mathrm{nM}$ (Figure 57B). Taken together, CRC cells with low USP22 levels can be specifically targeted by treatment with Ganetespib or the BET inhibitor JQ1.

In our aforementioned approaches, we tested the effects of transient USP22 loss in vitro in several human CRC cell lines by siRNA-mediated knockdown. However, this silencing is only transient and therefore only insufficiently reflects the in vivo situation of USP22 depletion. Thus, we decided to take advantage of the CRISPR/Cas9 gene editing technology to permanently delete the USP22 gene in HTC116 cells. The use of this construct allowed expression of a sgRNA sequence by the U6-promoter and at the same time expression of the Cas9 enzyme necessary for gene editing. For rapid selection of positively transfected cells, the plasmid contained a coding sequence for the Green Fluorescent Protein (GFP).We simultaneously transfected HCT116 cells with two constructs targeting intronic sequences flanking exon 3 to exon 5 of USP22, thereby allowing the excision of this segment. This generated a frameshift with as consequence deletion of functional USP22 gene (Figure 58). 


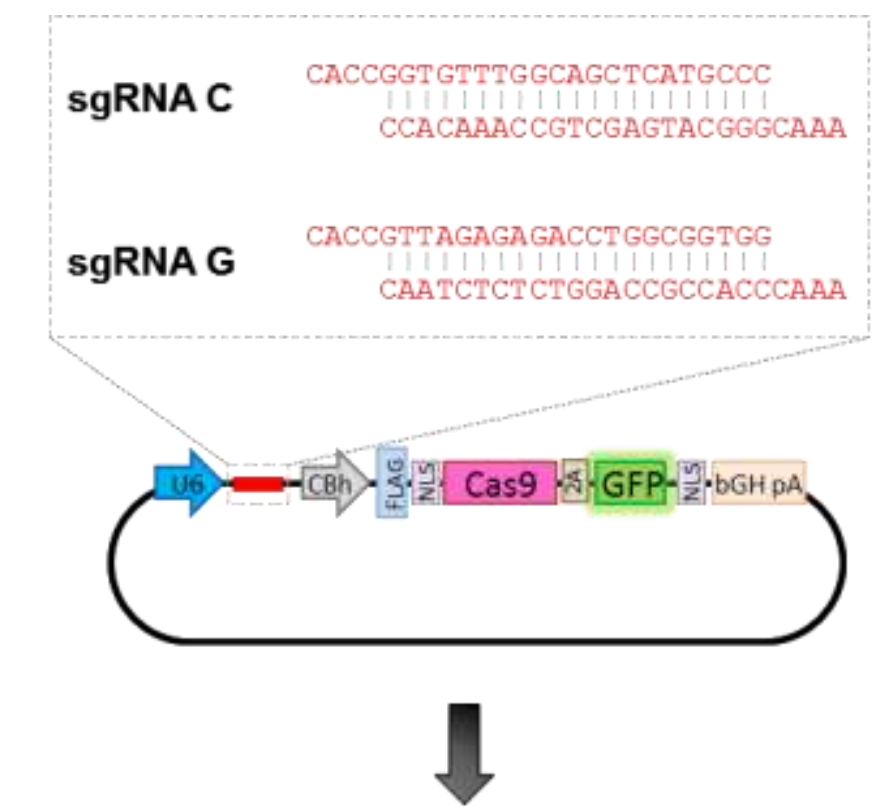

Figure 58: CRISPR/Cas9 approach to permanently deplete USP22 in HCT116 cells. HCT116 cells were transfected with two sgRNAs targeting USP22 (sgRNA "C" and "G") in a Cas9- and GFPcontaining vector. To allow selection, this construct contains a GFP-reporter. These sgRNAs target intronic regions upstream of exon 3 and downstream of exon 5 of the USP22 gene and generate a frameshift.

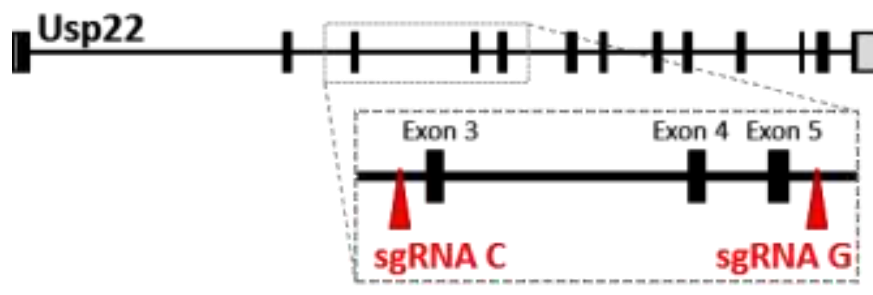

After $48 \mathrm{~h}$, we evaluated the transfection efficiency by detecting GFP-mediated fluorescence emitted by positively transfected cells (Figure 59A). We selected GFP-positive cells by fluorescence activated cell sorting (FACS) with the help of Sabrina Becker (Cell-sorting technology platform, Department of Haematology and Medical Oncology, UMG). Remarkably, with $32 \%$ fluorescent and 3\% highly fluorescent cells, the transfection efficiency of HTC116 cells was relatively high (Figure 59B). We decided to culture only highly GFP-positive cells (channel P4) as single cells in 96-well plates. 

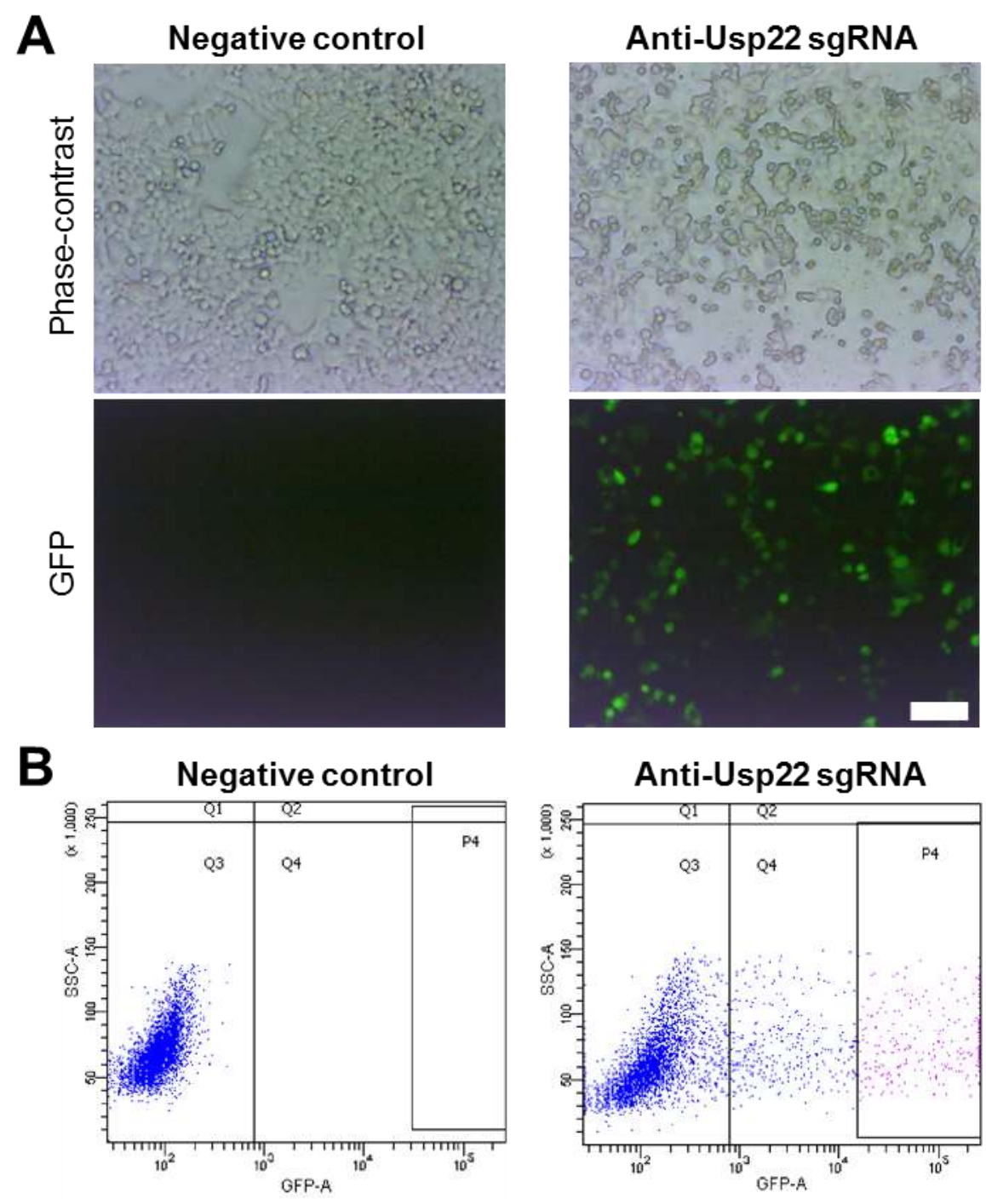

Figure 59: Fluorescent cells indicate the presence of the GFP-containing CRISPR/Cas9 constructs targeting USP22. (A) 48 hours after transfection GFP-positive cells were detected. Scale bar: 1,000 $\mu \mathrm{m}$. (B) Highly fluorescent cells (gate P4) were sorted as single cells by FACS.

After approximately four weeks of culturing and propagating single cells, several clones were tested for their USP22 levels. Two cell clones (\#1, \#2) did not show any residual USP22 protein (Figure 60A). As observed after siRNA-mediated knockdown, the loss of USP22 did not result in morphological alterations in HCT116 cells (Figure 60B). In addition, proliferation rates were assessed and again we could confirm previously obtained results in which reduced USP22 expression leads to accelerated cell growth in HCT116 cells (Figure 60C). Finally, we 
confirmed the sensitivity of USP22-depleted cells towards the HSP90 inhibitor Ganetespib (Figure 60D). Together, the effects of USP22 loss were reproduced in another cell system which represents a powerful tool to further investigate the consequences of a permanent USP22 depletion in human CRC cells.
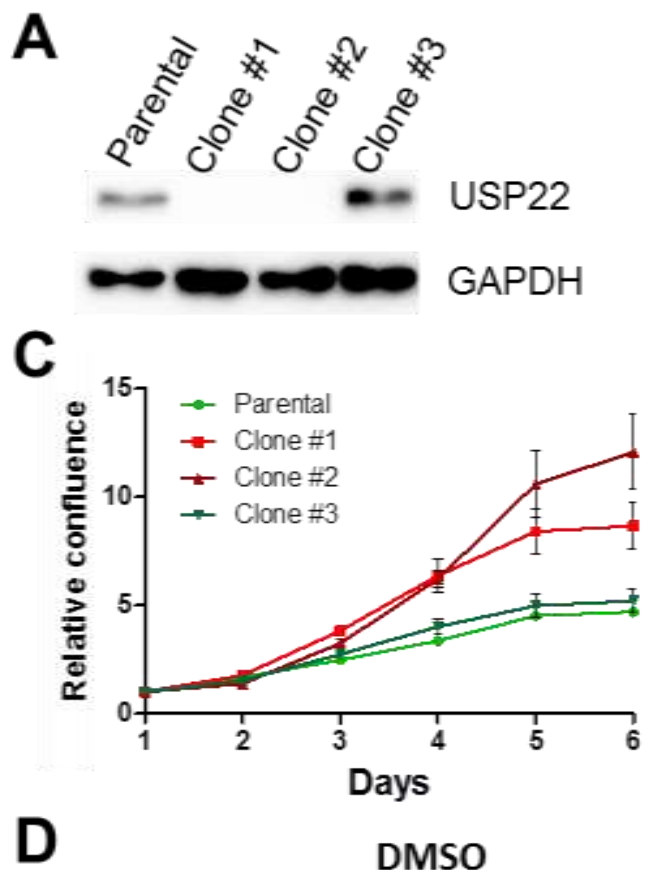

D

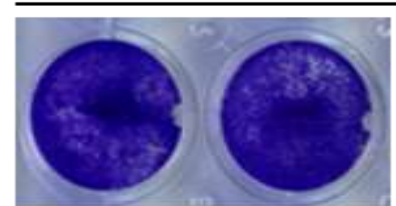

Parental

\#1
DMSO

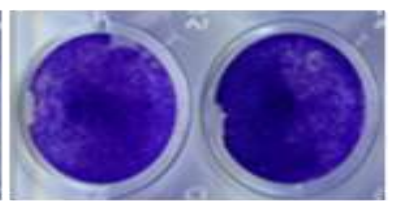

\#2
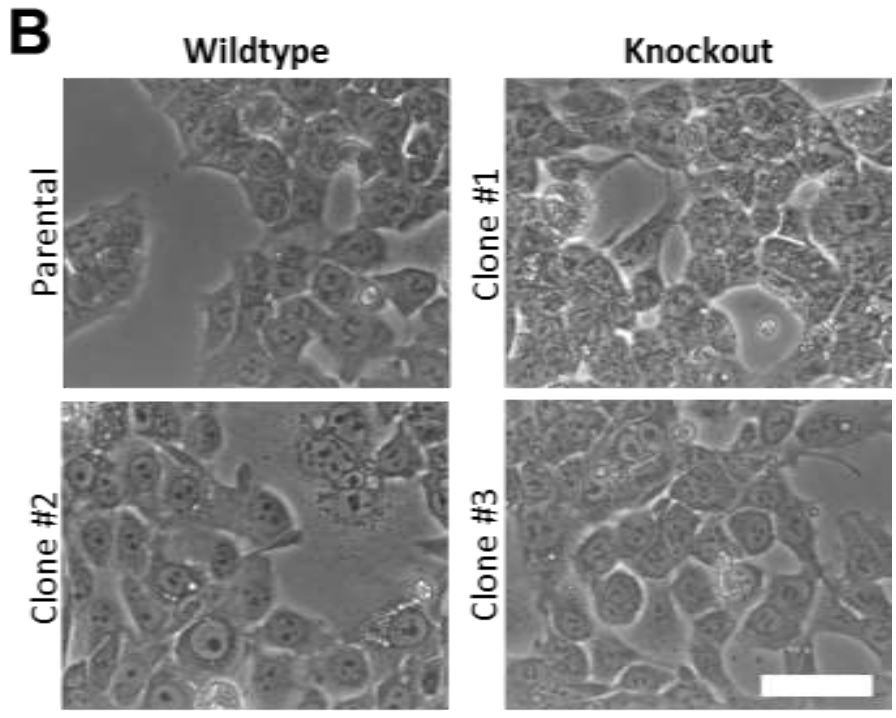

Ganetespib (333 nM)

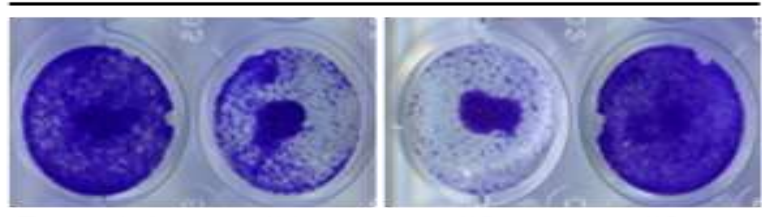

Parental
\#1
\#3

Figure 60: CRISPR/Cas9-mediated USP22 knockout leads to increased proliferation in HCT116 cells. (A) The loss of USP22 on protein level was confirmed in CRISPR/Cas9 clones \#1 and \#2. (B) As observed after siRNA-mediated knockdown the loss of USP22 does not affect the morphology of HCT116 cells. Scale bar: $100 \mu \mathrm{m}$. (C) Reduced USP22 expression results in increased proliferation compared to USP22 wild type levels (parental and CRISPR/Cas9 clone \#3 cells). Mean \pm SD, Student's $t$-test, $n=3$. (D) The sensitivity towards Ganetespib was confirmed in these USP22-depleted cells.

Together, in this project we demonstrated higher inflammation and tumor burden in mice with an intestinal deletion of Usp22. These findings were supported by data available in public databases in which a heterogeneous expression of USP22 was revealed in CRC patients. 
Accordingly, human CRC cell lines showed heterogeneous USP22 levels and reacted differently after USP22 knockdown with regards to proliferation and migration properties. mRNA-seq analyses indicated an involvement of USP22-regulated genes in proliferation- and differentiation-related processes as well as response to extracellular stimuli. Accordingly, we detected the downregulation of the heat shock protein HSP9OAB1 upon USP22 depletion in vitro. Interestingly, these cells showed increased sensitivity towards temperature changes and could be targeted by HSP90 and BET inhibitors based in the concept of synthetic lethality. Moreover, CRISPR/Cas9 cells with a USP22 knockout were generated which displayed high sensitivity towards HSP90 inhibition as well. These findings suggest a tumor suppressive function of USP22 and that low USP22 levels in CRC cells could be exploited by targeting these cells with specific inhibitors. 


\section{Discussion}

\subsection{USP22 is involved in differentiation and developmental processes in vivo}

Colorectal cancer is a global issue due to its high incidence and mortality rates. Thus, researchers worldwide aim to unravel underlying molecular signatures of this disease in order to generate and uncover more effective therapies. Despite USP22 overexpression was described in a number of human malignancies, its exact functions under physiological and pathological conditions remain unclear. In the current project we sought to investigate how USP22 functions in organ maintenance and colorectal tumorigenesis. To date, several studies on USP22 have been performed in vitro; however, in vivo data are limited. Interestingly, it was described by Lin et al. that the complete ablation of Usp22 expression results in early embryonic lethality at E10.5 of the post-implantation stage in mice (Lin et al., 2012). The mouse line Usp22 $2^{\text {lacz }}$ utilized in our studies displayed expression of approximately $3 \%$ of the correctly spliced Usp22 mRNA and can thereby be considered as a hypomorph. Due to the poor quality of commercially available antibodies, we were not able to establish immunohistochemical or immunofluorescent stainings to visualize reduced USP22 abundance on tissue sections. In addition, we repeatedly attempted to stain for Usp22 by in situ hybridization; however, no positive result was obtained. In future experiments utilization of the RNAscope ${ }^{\circledR}$ Technology, an in situ hybridization-based commercial assay, could help to confirm the reduction of Usp22 on tissue sections.

Using Usp22lacz animals we were able to visualize sites of Usp22 expression by making use of the LacZ reporter cassette. Notably, we could detect ubiquitous expression in the majority of tissues in embryos at E15.5. Its presence in the majority of tissues and its high abundance during embryonic development could explain why a complete loss of Usp22 has systemic consequences. In fact, we observed global growth retardation in adult Usp22 lacZlacz mice which 
weighed approximately $40 \%$ less than their wild type littermates. Previously, we were able to show that Usp22 $2^{\text {laczlacz }}$ mice were characterized by a differentiation shift in the small intestine, i.e. the number of OfIm4-positive stem cells and therefore the rate of differentiated Goblet, enteroendocrine and Paneth cells was increased (Kosinsky, 2013; Kosinsky et al., 2015). Accordingly, we detected similar differentiation shifts in the brain in which the number of precursor cells was decreased. These results suggest that differentiation processes in the intestinal epithelium and the cerebral cortex are partially regulated by USP22. Notably, while the gross morphology of the small intestine was not affected, the neuronal density within the cortex was decreased in Usp22 $2^{\text {lacZllacz }}$ animals. Therefore, we hypothesize that global differentiation and lineage specification, at least in some organs, are USP22-dependent. However, depending on tissue-specific cofactors, the consequences of Usp22 loss could vary. In order to investigate the alterations underlying the effects upon Usp22 reduction, we analyzed gene expression changes in mouse embryonic fibroblasts. Subsequent gene ontology analysis revealed an association of USP22 and differentiation-/proliferation-associated processes. Previously published data in murine embryonic stem cells (ESCs) support our findings since Usp22 expression was shown to be induced during ESC differentiation and to be required for proper differentiation into all three germ layers. The authors postulated that this function was mediated by USP22-associated $\mathrm{H} 2 \mathrm{~B}$ deubiquitination which controls transcription of the repressing factor Sex-Determining Region Y-Box 2 (SOX2) (Sussman et al., 2013). Consistently, our group demonstrated an increase of H2Bub1 levels during the differentiation of human mesenchymal stem cells (Karpiuk et al., 2012). However, in the present study, immunohistochemical stainings for H2Bub1 in the small intestine suggest that the effects of USP22 on differentiation are independent from its ability to deubiquitinate histone H2B since global H2Bub1 levels were unaffected by USP22 loss. Notably, a recent report from Atanassov and co-workers also revealed that global H2Bub1 levels were not elevated upon USP22 depletion but rather remain unchanged (Atanassov et al., 2016). Therefore, it would be possible 
that, depending on tissue-specific cofactors, USP22 exerts its function via H2B deubiquitination or that USP22 and H2Bub1 affect differentiation independent from one another. To address this question, studies in several tissues and cellular differentiation systems would be necessary. Taken together, the Usp22 ${ }^{\text {lacz }}$ mouse line enabled us to uncover a role of USP22 in cell differentiation and lineage specification in diverse organ systems. This observation was confirmed by microarray studies using MEFs from the same mouse line. Interestingly, these findings on differentiation may also be relevant in cancer since so-called cancer stem cells represent a subpopulation within a tumor and have been proposed to be able to self-renew and give rise to cells with divergent phenotypes. Thereby they can contribute to intratumoral cell heterogeneity and can be responsible for cancer initiation, progression and/or recurrence (Dawood et al., 2014; Zhang et al., 2015). Notably, in previous studies Glinsky described USP22 as a component of the 11-gene signature and speculated that this expression profile identifies lesions with cancer stem cell properties (Glinsky, 2005; Glinsky, 2006). Therefore, USP22 has been designated as a "putative cancer stem cell marker" in previous reports (Li et al., 2014b; Zhang et al., 2008b; Zhang et al., 2008a). Indeed, our data imply that USP22 could influence tumorigenesis by affecting differentiation properties of cells, possibly also affecting cancer stem cells. This hypothesis was supported by the mRNA-seq approach and subsequent GO analyses in human CRC cells which supported a function of USP22 in controlling differentiation-related genes in SW837 and SW480 cells.

\subsection{Intestinal Usp22 deletion causes mild spontaneous intestinal inflammation}

To evaluate the role of USP22 in intestinal tumorigenesis, we generated mice with a conditional intestine-specific ablation of Usp22. An additional APC truncation (APC ${ }^{1638 N}$ ) and/or DSSmediated colitis were utilized to promote tumorigenesis in these animals. To investigate the role of Usp22 in intestinal cancer formation in more detail, we made use of three different treatment options. There were two models for inflammation-induced CRC in which mice were 
treated with the chemical agent DSS to induce colitis. Experimental mice in the first cohort (Tam-DSS) were injected with Tamoxifen to induce the intestinal ablation of Usp22 and afterwards DSS was administered. This cohort allowed us to observe the consequences of Usp22 loss on colitis burden and subsequently on the formation of inflammation-induced tumors. In contrast, the DSS-Tam animals were treated with DSS first, and four weeks later, Usp22 was deleted. In this cohort we could ensure that the colitis burden is comparable in all animals and that inflammation intensity was not affected by an intestinal-specific Usp22 loss. Finally, we determined the effect of Usp22 ablation in a model for sporadic colorectal cancer formation. For this purpose, mice were injected with Tamoxifen, but were not treated with DSS (Tam cohort).

Health parameters were checked on a regular basis, including body weight, stool consistency and intestinal bleedings. In the majority of mice, DSS treatment resulted in weight loss, diarrhea and bleedings. Especially in the Tam-DSS cohort, APC ${ }^{1638 \mathrm{~N} /+}$, Usp22 $2^{-/-}$mice displayed an increased burden. It should be mentioned that also in wild type mice not treated with DSS (Tam cohort) intestinal bleedings were detected according to the Guaiac test results. It has been reported that the risk of false-positive outcomes is high using this method (Roslani et al., 2012). However, even when considering this false-positive "background", APC ${ }^{1638 \mathrm{~N} /+}$, Usp22 $2^{-/-}$animals showed increased bleeding intensities, including bloody anuses. Mice with this genotype were frequently affected by increased loss of body weight. Moreover, we detected that the colons in $\mathrm{APC}^{1638 \mathrm{~N} /+}$, Usp22 $2^{-/-}$animals were shorter than in wild type littermates. In a study on inflammatory bowel disease patients it was detected that individuals with ulcerative colitis had significantly shorter intestines compared to the healthy control group (Nordgren et al., 1997). Therefore, the decreased colon length in APC ${ }^{1638 \mathrm{~N} /+}$, Usp22 $2^{-/-}$mice is likely indicative of severe inflammatory burden. In previous studies it was reported that depending on the mouse strain, recovery and complete disappearance of symptoms was observed approximately 4-5 weeks after DSS administration (Chassaing et al., 2014). In both cohorts used for inflammation- 
induced CRC (Tam-DSS and DSS-Tam) the DSS treatment was terminated when animals were 12 weeks old. Interestingly, APC ${ }^{1638 N /+}$, Usp22 $2^{-/}$animals mice had to be sacrificed at an age of 12-20 weeks in the Tam-DSS and 18-40 weeks in the DSS-Tam cohort. Therefore, we can postulate that several APC ${ }^{1638 \mathrm{~N} /+}$, Usp22 $2^{-/}$mice in the Tam-DSS group may have still been affected by the acute inflammation when they died. However, some mice with this genotype died when the symptoms of colitis should have disappeared. Surprisingly, in the histological assessment of $\mathrm{APC}^{1638 \mathrm{~N} /+}$, Usp22 $2^{-/}$colons, all mice were affected by similar rates of inflammation regardless of whether they died during the acute phase of colitis or when symptoms should have already disappeared. Morphological observations made in all these mice (i.e. shortening of colons) supported the conclusion that the inflammation was not resolved in these animals. In addition, we detected severe lymphocyte accumulation upon the loss of Usp22. In fact, large mucosal segments were infiltrated by immune cells resulting in increased H-scores. Remarkably, even mice not treated with DSS at all (Tam cohort) were characterized by mild inflammatory symptoms upon Usp22 knockout. These findings suggest that an intestinal knockout of Usp22 results in prolonged inflammatory reactions upon DSS treatment and/or a slowed recovery from inflammation. Moreover, we assume that the loss of Usp22 maintains a latent inflammatory environment in general, even without DSS treatment. As a consequence, upon additional induction of colitis, an excessive immune response is triggered which is further maintained in Usp22 ${ }^{-}$animals even after symptoms have disappeared in wild type mice. This may not only happen due to an excessive immune response but may also be the result of attenuated feedback mechanisms controlling/terminating the inflammatory reaction. Generally, delayed resolution of inflammation was associated with, for instance, increased immune cell infiltration, reduced stimulation of tissue repair or inefficient efferocytosis (Ortega-Gomez et al., 2013). However, whether one of these processes underlies the elevated inflammatory response in Usp22- mice is not yet clear and should be addressed in future studies. 
As a consequence of intestinal inflammation, the crypt integrity is compromised which interferes with the normal colonic functions including nutrient absorption (Xavier and Podolsky, 2007). Notably, it was demonstrated that children suffering from inflammatory bowel diseases commonly exhibit growth defects due to chronic inflammation and resultant malnutrition (Sanderson, 2014). This epiphenomenon of latent inflammation could, besides the role of USP22 in differentiation, explain the phenotype of Usp22 $2^{\text {lacz/lacz }}$ mice which were characterized

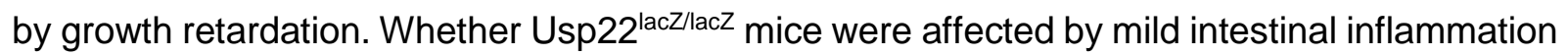
was not tested in those studies and remains to be elucidated.

To obtain more insights into the impact of Usp22 loss in inflammation, we performed in vivo analyses using an acute colitis model. Two days after terminating the DSS treatment, thus during the acute phase of colitis, mice were sacrificed. As expected, the disease activity index (DAI) including weight loss, stool consistency and intestinal bleedings was elevated in Usp221- animals. It was previously described that a heterozygous APC mutation leads to anemia and increased spleen size (Qadri et al., 2012). Consistently, the ratio of hematocrit in blood samples was decreased in APC ${ }^{1638 \mathrm{~N} /+}$ animals. Moreover, these mice possessed pale livers, kidneys as well as bigger and heavier spleens. Notably, this phenomenon was slightly aggravated by the loss of Usp22. Importantly, strong inflammatory reactions were described to modulate bone mineral density and increase fracture risk (Ali et al., 2009; Bernstein et al., 2000). Thus, we measured biomechanical properties of bones and observed more fragile bones in $\mathrm{APC}^{+/+}$, Usp22 $^{-/-}$animals. Previously, it was shown that APC mutations increase bone mineral density (Miclea et al., 2010). However, we could not detect any differences in bone strength between APC wild type and $\mathrm{APC}^{1638 \mathrm{~N} /+}$ animals with USP22 wild type status. In fact, the majority of mice had similar bone strengths except for $\mathrm{APC}^{+/+}, \mathrm{Usp}^{2} 2^{-{ }^{-}}$animals. It is not clear why the knockout of Usp22 alters bone characteristics only in $\mathrm{APC}^{+/+}$but not $\mathrm{APC}^{1638 \mathrm{~N} /+}$ mice. To verify these findings and to obtain further insights into the role of Usp22 in inflammation-induced bone fragility, mouse cohorts with increased n-numbers and a control group which is not treated with 
DSS are necessary. In addition, computed tomography scans should be performed to obtain further insights into bone structure and properties. Together, our results reveal that intestinal Usp22 loss enhances inflammation burden in DSS-treated mice, and, remarkably, can even cause spontaneous inflammation. Notably, the colitis burden was sufficient to induce inflammation-associated bone fragility in Usp22 $2^{-/}$mice. The latent inflammatory environment in Usp22-- animals could be involved in growth retardations in Usp22 laczlacz animals and favor colorectal tumor formation.

To reduce burden, IBD patients are frequently treated with anti-inflammatory drugs such as aminosalicylates and corticosteroids or immunosuppressant drugs including azathioprine, cyclosporine and TNFa inhibitors (e.g. Infliximab) (Baumgart and Sandborn, 2007). The efficacy of several of these drugs was also demonstrated in DSS-induced colitis in mice (Fukata et al., 2011; Kim et al., 2010; Myrelid et al., 2015; Sann et al., 2013). However, long-term usage of these drugs can have adverse effects, for instance on the intestinal microbiota (Rogers and Aronoff, 2016), an aspect of intestinal inflammation which has not been considered in this project. As mentioned before, dysbiosis is associated with colitis (Rehman et al., 2010). Initially, to test whether the microbiota is involved in the intestinal tumor development, it would be possible to treat the mice with antibiotics. As demonstrated by Peuker and colleagues, changing the composition of the microbiota by antibiotic treatment can inhibit the intestinal tumor growth in $\mathrm{Apc}^{\mathrm{min} /+}$ mice (Peuker et al., 2016). Moreover, identifying and comparing the composition of the microbiota in Usp22 wild type and knockout animals could be informative and could potentially be correlated to the inflammation intensity in these animals. This identification could, for instance, be implemented by $18 \mathrm{~S}$ rRNA sequencing of the microbiome (Dollive et al., 2012). Moreover, Souza and co-workers demonstrated elegantly how the supplementation of certain microorganisms can alleviate the effect of colitis in mice (Souza et al., 2016). Initially, they induced colitis in animals and observed that upon administration of the Escherichia coli strain Nissle 1917 (EcN), the colitis burden was decreased. Afterwards, they 
applied the fecal content of EcN-treated animals to germ-free mice which were subsequently subjected to colitis. Indeed, these mice were protected from colitis-induced symptoms indicating to what extent bacteria can influence susceptibility to IBDs. Studies like this suggest that fecal microbiota transplantations (FMTs) present promising treatments for IBD patients. In a recent meta-analysis, it was demonstrated that FMTs lead to clinical remission in $22 \%$ of UC and $60.5 \%$ of CD patients (Colman and Rubin, 2014). To what extent anti-inflammatory drugs or FMTs could ameliorate the effects of Usp22 loss-induced inflammation and therefore lead to a lower tumor burden would be an interesting research question remaining to be elucidated.

\subsection{Intestinal Usp22 loss promotes inflammation-induced and sporadic CRC}

After measuring the colon length, the number of intestinal tumors was counted and the location of cancer lesions was determined. To our surprise, Usp22 $2^{--}$mice were characterized by an elevated tumor burden in both the small intestine and colon. Notably, the observation that Usp22 promotes intestinal tumor formation was made in all three experimental cohorts, i.e. Tam-DSS, DSS-Tam and Tam. This finding emphasizes the need to compare the three experimental cohorts in more detail to determine the impact of Usp22 in inflammation-induced (via DSS treatment) and sporadic CRC. When comparing the two groups for inflammationinduced CRC, it is clear that the Tam-DSS treatment caused more tumors in all genotypes in both the small intestine and colon. In fact, the tumor incidence almost doubled in this group. As mentioned before, Usp22 loss alone (Tam cohort) resulted in mild latent inflammation, which could be the underlying reason for the exacerbated inflammatory processes in Tam-DSS mice. In contrast, DSS-Tam mice did not experience the early loss of Usp22. Instead, the burden of colitis was similar in all animals and Usp22 was inactivated during or shortly after the process of epithelial healing. In other words, after Usp22 knockout, inflammatory processes were initiated in Tam-DSS animals and exacerbated during colitis while DSS-Tam mice did not 
develop this latent inflammation before DSS treatment. Consequently, Tam-DSS animals developed more intestinal tumors than DSS-Tam mice.

As expected, mice only treated with Tamoxifen (Tam cohort) had the lowest tumor burden in the colon probably because they did not undergo colitis. Interestingly, Usp22 $2^{-/-}$mice in the Tam group showed the highest abundance of tumors in the small intestine. Moreover, several tumors were detected in the colons of these animals. These findings were surprising since they indicate that the loss of Usp22 alone is sufficient to trigger sporadic tumor formation; mainly in the small intestine. This observation supports our hypothesis that the loss of Usp22 triggers a latent inflammation in the intestinal system which consequently promotes tumorigenesis. Therefore, one should keep in mind that, if Usp22 loss alone can maintain a mild latent inflammation, the tumor formation in our "sporadic" Tam cohort is most likely, at least to some extent, also inflammation-induced.

Interestingly, the three experimental cohorts showed varying tendencies when evaluating the tumor burden in the different intestinal segments. As mentioned before, Tam-DSS was associated with the highest tumor number in the colon. In contrast, in the small intestine, Tam mice were characterized by the most severe tumor burden, especially in APC ${ }^{1638 N /+}$ mice. Generally, colitis exerts strongest effects in the colon (Adams and Bornemann, 2013) which could explain why the Tam-DSS group shows most cancer lesions in the colon. However, it is challenging to clarify why the tumor burden in the small intestine is highest in the Tam cohort. One possible explanation is based on the assumption that Usp22 loss alone results in a latent inflammatory environment which finally leads to tumorigenesis. When we expect that the APC mutation is mainly correlated with tumor growth in the proximal region, it is likely that immune cells migrate to the sites of oncogenic growth in the proximal intestinal regions (Fodde et al., 1994). This lymphocyte infiltration in turn can promote tumorigenesis in these segments. Indeed, it was reported that immune cells are recruited to cancer lesions where they produce cytokines and chemokines and thereby accelerate oncogenic growth (Klampfer, 2011). 
Assuming that intestinal Usp22 loss promotes latent inflammation, it can be expected that the rate of infiltrating lymphocytes is increased in Usp22 $2^{-/-}$animals, which causes an elevated tumor burden in the small intestine. In contrast, if $\mathrm{APC}^{1638 \mathrm{~N} /+}$, Usp22/- mice are subjected to DSS treatment there will be lymphocyte accumulations at the site of acute inflammation, i.e. the colon, but a portion of lymphocytes will still migrate to the site of $\mathrm{APC}^{1638 \mathrm{~N}}$-associated tumorigenesis, i.e. the proximal SI. This hypothesis would explain the accumulation of tumors in the proximal/medial small intestine segments and the colon of APC ${ }^{1638 \mathrm{~N} /+}$, Usp22-/- mice. The phenomenon that there was an accumulation of tumors in the proximal and medial segments of the small intestine could be explained by further scenarios. First, the aforementioned abundance of the APC mutated tumors in the proximal intestinal region (Fodde et al., 1994) could have been augmented by the additional loss of Usp22. Thus, the interplay between APC mutation and Usp22 ablation would be the driving factor here. Moreover, the Villin promoter is highly active in the upper intestine. Thus, it is possible that the cells in this region were strongly affected by the Usp22 loss and therefore, the rate of Usp22 loss-related tumorigenesis was increased independent of APC mutation. Here, it would be imaginable that this phenomenon is HSP90-dependent. In our in vitro studies we noticed increased temperature sensitivity of USP22 knockdown cells, possibly due to the USP22 loss-related degradation of the heat shock protein HSP90AB1. Assuming that HSP90AB1 levels are also decreased in intestinal epithelial cells in Usp22 $2^{-/-}$animals, these cells would display a higher sensitivity towards inflammation-induced stress. Thus, these cells would be prone to stressrelated apoptosis or necrosis, which favors and exacerbates inflammation (Davidovich et al., 2014) and therefore promotes tumorigenesis, for instance, by the production of reactive oxygen and nitrogen species (Meira et al., 2008) as mentioned in section 1.5. Since the activity of the Villin promoter is highest in the proximal SI region, cells located there are strongly affected by USP22 loss and HSP90AB1 reduction. This would potentially explain the increased tumor abundancy in the upper SI segment. 
Importantly, the tumors in Usp22-deficient mice were shown to be more aggressive. Invasive tumors could exclusively be found in Usp22 $2^{-1}$ animals, independent from the APC status. Notably, this increases the risk of metastasis and is therefore associated with a significantly poorer prognosis (O'Connell et al., 2004). However, we could not detect any metastases in our experimental mice which, nonetheless, could still mean that microinvasion has taken place. In addition to invasive adenocarcinomas, we made another interesting observation. The loss of Usp22 was correlated with the presence of intramural mucus accumulations and remarkably, tumors with a mucinous appearance. Interestingly, patients with colitis-associated CRC frequently display mucinous lesions (Higashi et al., 2011) regularly due to a high abundance of the mucin components MUC1, MUC2 and MUC5AC (Slotkin and Seidler, 1975). Notably, Usp22laczlacz mice were characterized by an increased abundance of MUC2-positive Goblet cells. Therefore, it is likely that APC ${ }^{1638 N /+}$, Usp22-/- mice show a similar increase in Goblet cells; however, this still needs to be verified by IHC. As mentioned before, mucinous presentation is generally correlated with a poor prognosis compared to normal adenocarcinomas (Verhulst et al., 2012). Since mucinous carcinomas are frequently microsatellite instable, a PCR-based MSI analysis would have been interesting to obtain further insight into the pathology of tumors formed upon the loss of Usp22. The detection of MSI in murine samples based on PCR and capillary electrophoresis has already been described (Bacher et al., 2005; Kabbarah et al., 2003) and would be an appropriate approach to detect the rate of mono- or dinucleotide repeats upon Usp22 loss. Together, we could demonstrate that the loss of Usp22 promotes sporadic and inflammation-induced colorectal tumorigenesis; however, further analyses are needed to reveal tumor characteristics. 


\subsection{Intestinal Usp22 deletion in APC ${ }^{1638 \mathrm{~N} /+}$ animals decreases life span}

One of the key observations made in this project was the short life span of Usp22-deleted mice. Already during the first DSS treatments which we performed (Tam-DSS cohort), we could measure severe weight loss and intestinal bleedings in APC ${ }^{1638 \mathrm{~N} /+}$, Usp22 $2^{-/}$mice. This observation was confirmed in DSS-Tam animals. In particular, shortly before the mice needed to be sacrificed we noticed worsening of the health status, i.e. swollen abdomen, high weight loss, pale extremities, rough fur and limited movements. When APC ${ }^{1638 N /+}$, Usp22-- mice were sacrificed due to their physical condition we regularly detected intussusceptions and rectal prolapses. Generally, an intussusception reflects a condition in which a proximal intestinal part invaginates into a distal segment. This phenomenon can be observed when the peristaltic movement is disturbed, for instance, due to a large tumor (Honjo et al., 2015), and can result in an acute intestinal obstruction (Tan et al., 2003). Similarly, a rectal prolapse describes an intussusception of the rectal segment through the anus (Hatch and Steele, 2013). In patients, late diagnosis can lead to intestinal perforation with consequent peritonitis and eventually sepsis, which results in an increased mortality risk (Udo et al., 2016).

In our study, the detection of intussusceptions and prolapses already indicated the presence of tumors in Usp22 $2^{-/}$animals. After sacrificing mice, intussusceptions were predominantly found in the proximal region of $\mathrm{APC}^{1638 \mathrm{~N} /+}$ animals, where the frequency of tumors in proximal intestinal segments has been described before (Fodde et al., 1994). Indeed, within SI regions affected by intussusceptions, large tumors were found.

Regarding survival and tumor burden in the SI, similar observations were made in mice which were not treated with DSS (Tam group), however, lethality was clearly accelerated by colitis. In general, the survival rates of $\mathrm{APC}^{+/+}$animals were nearly unaffected and animal mortality was infrequent. Moreover, outcomes of Usp22 wild type and heterozygous animals were similar in all three treatment cohorts and no statistically significant variations between these two genotypes were observed. Notably, the Tam-DSS group was associated with the shortest 
survival of APC ${ }^{1638 N /+}$, Usp22-/- mice while only marginal differences were detected between the DSS-Tam and Tam mice.

Our data imply that an intestinal loss of Usp22 causes a latent inflammation in the small intestine and colon. This inflammatory state likely accelerates tumorigenesis, which subsequently induces early lethality in $\mathrm{APC}^{1638 \mathrm{~N} /+}$, Usp $22^{-/-}$mice, frequently due to intussusceptions or prolapses. Therefore, we hypothesize that treating these animals with antiinflammatory drugs or antibiotics could lower inflammation and tumor burden. Even though CRC-related death could not be prevented completely due to the APC mutation, this measure would be likely to extend survival rates.

\subsection{The heterogeneity of USP22 expression in colorectal cancer}

In recent years a significant amount of gene expression and mutation data has become publically available. Analyses of these data provide an excellent source of information on gene expression profiles, mutation occurrences and patient survival in the field of cancer research. In this study, we took advantage of several databases, i.e. Oncomine ${ }^{\mathrm{TM}}$, cBioPortal, and the IST Online ${ }^{\circledR}$ database, to evaluate USP22 expression levels in colorectal cancer patient samples. Based on previously published studies in which USP22 overexpression was detected in several cancer types (Melo-Cardenas et al., 2016), we expected to detect elevated USP22 levels in CRC patients using these online tools. Remarkably, analyses of all databases led to the conclusion that USP22 expression is not as 'black and white' as described in previous studies. In fact, approximately half of listed CRC patients displayed decreased expression of USP22. Consistently, USP22 mRNA levels were found to be decreased in several subgroups of cecal, colonic or rectal cancer in another database. Interestingly, some CRC datasets available on cBioPortal revealed a heterozygous deletion of the USP22 gene in 44-49\% of all colorectal cancer patients. In comparison, the well-known tumor suppressor TP53 was heterozygously deleted in $51-57 \%$ and $A P C$ in $19-25 \%$ of the patients. This underlines the 
possibility that USP22 may serve as a tumor suppressor depending on the biological context. Accordingly, USP22 protein levels were highly variable in a panel of colorectal cancer cell lines. These cells were subsequently transfected with siRNAs targeting USP22 in order to test the effect on morphology and proliferation after depletion. While some cell lines were not affected by the loss of USP22, in most cells the knockdown resulted in decreased proliferation. Interestingly, growth was enhanced in HCT116 cells, which may therefore more closely reflect the condition observed in our in vivo approaches, i.e. aggressive growth of colorectal cancer cells upon USP22 depletion. In contrast, Xu and co-workers reported decreased cell proliferation following USP22 knockdown in HCT116 cells (Xu et al., 2012). Since we could reproduce our findings numerous times, we considered our findings as being reliable. After these Celigo ${ }^{\circledR}$ measurements, cell line characteristics such as MSI/CIN/CIMP status, were evaluated in order to elucidate whether they could be correlated with the proliferation pattern of siUsp22 cells. Since we did not detect any correlations, we also considered mutation profiles of the cell lines, however, no connection could be made. This heterogeneity was further confirmed when testing the migration potential upon USP22 knockdown. While the migration potential was inhibited in SW48 cells, we observed the opposite effect in HCT116 cells. Meanwhile we aimed to verify that the effects we observed were mediated by a reduction of USP22 alone and not by siRNA-associated off-target effects in which the homolog USP27X is targeted. For this purpose, we performed individual knockdowns of USP22, USP27X as well as both USPs simultaneously in HCT116 and SW48 cells. While none of the conditions showed an effect on cell morphology, as expected, USP22 reduction resulted in decreased cell growth in SW48 cells. Moreover, in this cell line USP27X loss and the dual knockdown showed similar effects to USP22 ablation. In contrast, in HCT116 cells USP22 loss enhances proliferation while siUsp27x cells displayed severely impaired proliferation. Notably, the dual knockdown could rescue the devastating effect of USP27X on cell growth. Since the siRNAs targeting USP22 and USP27X, respectively, had highly different effects on proliferation, we could rule 
out the possibility that the USP22-siRNAs show off-target effects by modulating USP27X levels. Interestingly, our proliferation measurements suggest that if loss of USP27X decelerates the growth of CRC cells, it functions as an oncogene. In contrast, the additional loss of USP22 rescues this effect, implying that it serves as a tumor suppressor in this context. This assumption is supported by a recent report in which the shRNA-mediated loss of USP27X resulted in decreased growth of breast cancer cells in a xenograft approach (Atanassov et al., 2016). In contrast, in non-small cell lung cancer cells, USP27X was described to act as a tumor suppressor in vitro (Weber et al., 2016). Thus, the effects which we have observed in HCT116 may be cell line-specific and possibly, the role of USP27X is just as context-specific as the function of USP22 in tumorigenesis.

\subsection{The interaction between USP22 and HSP90AB1}

To obtain further insights into the mechanisms underlying the effect of USP22 in CRC cells, we performed mRNA-seq in SW837 and SW480 cells. These cell lines were selected since they underwent only marginal changes upon USP22 knockdown. Therefore, we expected that the secondary effects caused, for instance, by dramatic changes in cell phenotype, would be minimal and negatively influence results. We were able to confirm that $H S P 90 A B 1$ is downregulated in siUsp22 cells. Generally, HSP90 family members are involved in cellular adaptation to stress (Whitesell and Lindquist, 2005). While the stress-inducible HSP90a isoforms were not affected by USP22 depletion, the constitutively expressed HSP90ß member HSP90AB1 was downregulated. Consistently, when we performed heat shock assays, we demonstrated that siUsp22 cells possessing decreased HSP90AB1 levels are characterized by elevated temperature sensitivity. Generally, increased expression of HSP90 family members correlated with human malignancies (McDowell et al., 2009) and a variety of HSP90 inhibitors has been tested in clinical trials (Solarova et al., 2015). Surprisingly, when analyzing the frequency of heterozygous deletions using cBioPortal it was determined that $H S P 90 A B 1$ is 
affected in $3-8 \%$ of $\mathrm{CRC}$ patients and in up to $30 \%$ of individuals suffering from other cancers. Thus, similar to USP22, the levels of HSP90AB1 in CRC can be heterogeneous. When correlating these findings to our in vivo results indicating that Usp22 deletion contributes to intestinal tumorigenesis, there are three possible scenarios. First, it would be imaginable that the tumor-promoting effect of USP22 ablation is in part mediated by the resultant HSP90AB1 reduction. Second, it is imaginable that the reduction of HSP90AB1 via USP22 ablation does not only sensitize cells to elevated temperatures but also to inflammatory signaling which can also be considered as stress. This scenario would support our hypothesis based on our in vivo approaches in which Usp22 loss was associated with increased inflammation. If USP22 has a general role in protecting the cell from stress-induced factors, Usp22 knockout mice could be affected by increased inflammation burden. Consequently, the risk of developing tumors would increase in these animals. While several studies published so far show that the inhibition of HSP90 suppresses inflammation-associated processes (Lilja et al., 2015; Zhao et al., 2013), a dual role of HSP90, protective and cytotoxic, was observed as well (Paepe et al., 2009). In order to confirm this assumption the HSP90AB1 levels should be determined in mouse tissue, i.e. by western blot, qRT-PCR and IHC. A third possibility is that the reduction of HSP90AB1 in our in vitro experiments is a bystander effect and is not responsible for the effects we observe following USP22 loss. Instead, another unknown factor may be associated with the tumor suppressor activity of USP22.

Subsequent analysis of the functional interaction by Co-IP revealed that USP22 and HSP90AB1 form a complex. Our finding was supported by a proteomics-based study in which several heat shock protein 70 members were identified as USP22 interaction partners (Sowa et al., 2009). Afterwards, by evaluating HSP90AB1 levels upon treatment with the proteasome inhibitor MG-132, we could demonstrate that decreased HSP90 amounts in siUsp22 cells are dependent upon proteasomal degradation of this protein. Due to the physical interaction and the temperature sensitivity of siUsp22 cells we speculated that cancer cells with low USP22 
levels are highly dependent on the residual HSP90 expression. Consequently, we aimed to take advantage of low HSP90 levels created by USP22 loss to induce synthetic lethality with the help of the HSP90 inhibitor Ganetespib. Indeed, we could observe that cells with a siRNAand CRISPR/Cas9-mediated depletion of USP22 are more sensitive to Ganetespib. Based on this finding we hypothesize that under physiological conditions, USP22 deubiquitinates HSP90 family members and thereby stabilizes them (Figure 61). Subsequently, HSP90 can facilitate the formation of the P-TEFb complex which will be recruited by BRD4 to phosphorylate the RNA Pol II. In contrast, the loss of USP22 leads to the poly-ubiquitination of its substrate HSP90, resulting in its proteasomal degradation. Consequently, formation rates of the P-TEFb complex would be decreased. Thus, P-TEFb binding rates to BRD4 and therefore RNA Pol II phosphorylation-associated transcriptional elongation would be reduced. In turn, transcription levels of HSP90 and further genes potentially preventing tumorigenesis would be reduced. 


\section{A}

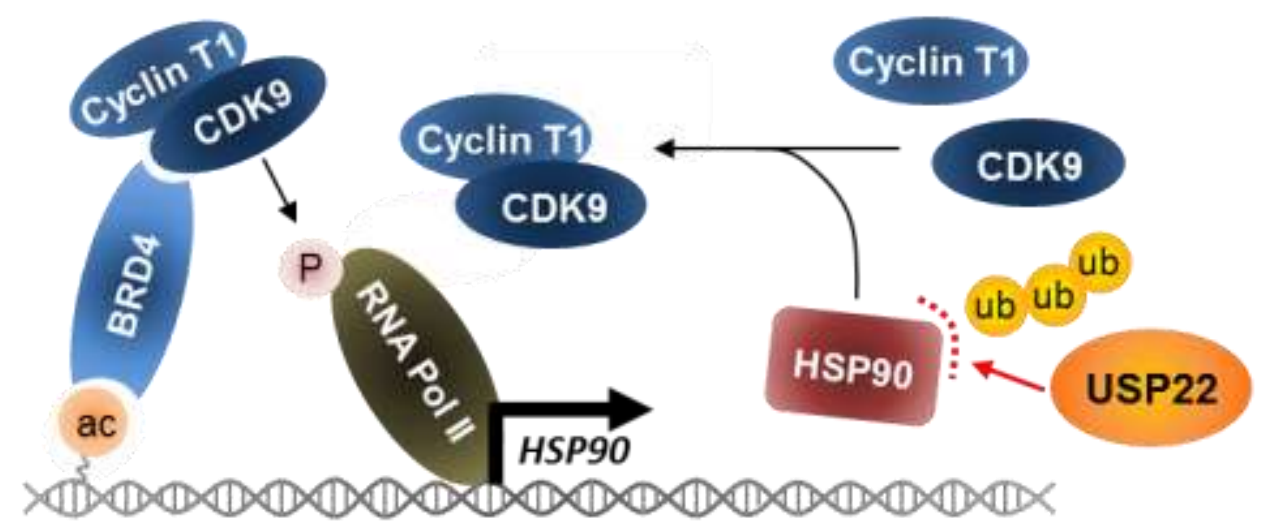

B

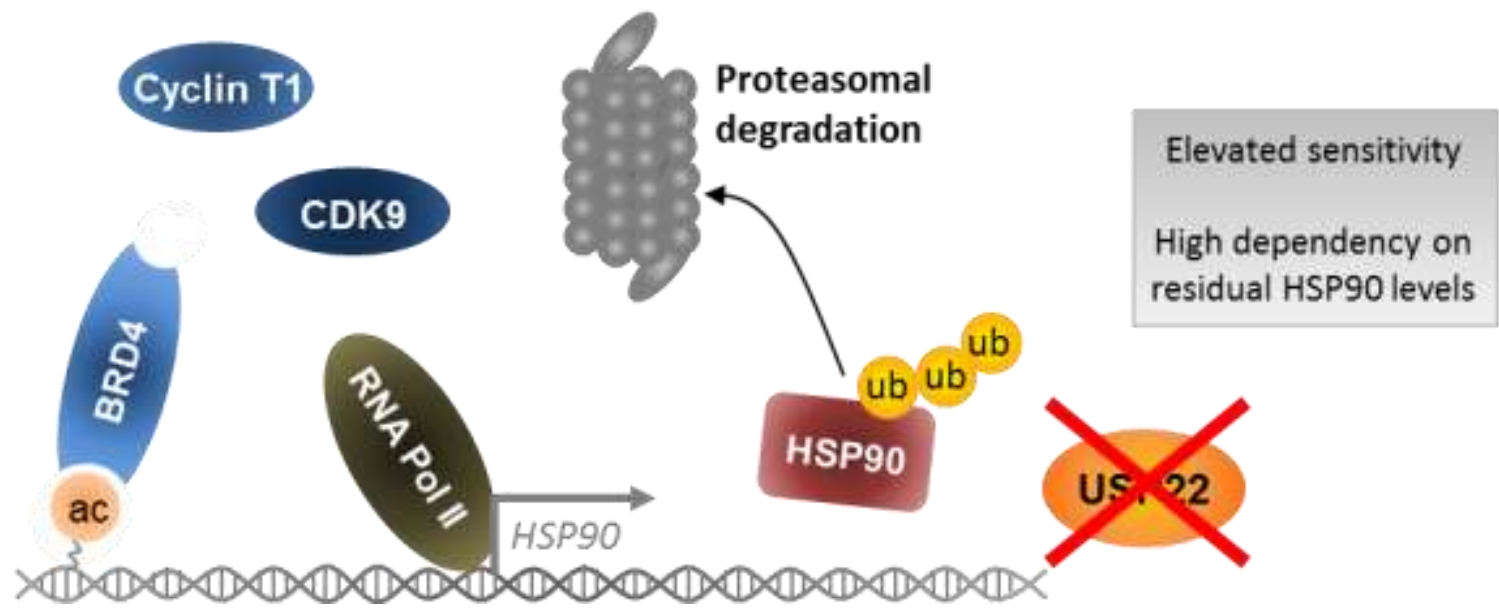

Figure 61: Proposed model of the effect of USP22 loss on HSP90 levels. (A) Under normal conditions, HSP90 facilitates the formation of the P-TEFb complex. Upon recognition of acetylated marks, BRD4 recruits P-TEFb and CDK9 phosphorylates the RNA Pol II. Thereby promoter proximal pausing is released and transcription, e.g. of $H S P 90 A B 1$, is promoted. (B) In the absence of USP22, polyubiquitination rates of HSP90 increase, resulting in its proteasomal degradation. As a consequence, the P-TEFb complex formation is diminished, leading to lower RNA Pol II phosphorylation and decreased transcriptional elongation. Reduced HSP90 levels elevate the cells' sensitivity towards stress stimuli and therefore, cells are highly dependent on the residual HSP90 rates.

Based on this potential model, we tested the effect of the BET inhibitor JQ1. Normally, HSP90 facilitates the assembly of the P-TEFb complex which is subsequently recruited by the BET protein BRD4. We proposed that after the Ganetespib-mediated inhibition of HSP90, the PTEFb stability and therefore its binding to BRD4 is reduced. Consequently, by inhibiting BRD4 using JQ1 and thus preventing the recruitment of PTEF-b to chromatin, we expected to observe 
similar effects in siUsp22 cells as after Ganetespib treatment. Interestingly, BRD4 has also been identified as a HSP90 client which is stabilized by this heat shock protein (Zhou et al., 2015). Indeed, following JQ1 treatment we could detect increased sensitivity of siUsp22 cells towards this small molecule inhibitor. While it is not completely clear why synthetic lethality was induced using JQ1, there are two possible scenarios. First, it is imaginable that, similar to HSP90, BRD4 is normally deubiquitinated by USP22 and that siUsp22 cells are dependent on the residual BRD4 levels. Moreover, this stabilization could be mediated by HSP90 as previously described (Zhou et al., 2015) and therefore indirectly by USP22. Indeed, we could detect sporadic BRD4 reduction upon depletion of USP22. However, this effect was highly variable. Another possibility could be that by the inhibition of BRD4, the transcription patterns of several genes are affected and that, among others, HSP90 expression is further decreased. The reduction of HSP90 can then result in decreased P-TEFb complex assembly which leads to reduced phosphorylation of RNA Pol II. Thus, the release of promoter proximal pausing was decreased which can affect a variety of genes. Taken together, we speculate that siUsp22 cells are dependent on their low HSP90 and possibly BRD4 levels and a further decrease by Ganetespib or JQ1 is lethal for the cells. In future experiments this could be verified by testing BRD4 protein levels upon Ganetespib treatment. Alternatively, gene expression profiles after JQ1 and Ganetespib treatment as well as USP22 knockdown could be identified by mRNAseq and subsequently compared to evaluate the overlap of affected genes between these conditions. In summary, even if the reduction of HSP90AB1 is only a bystander effect after USP22 ablation, these outcomes demonstrate that it is possible to exploit USP22/HSP90AB1 reduction in CRC cells and to target them based on this characteristic making use of the concept of synthetic lethality. 


\subsection{USP22: oncogene or tumor suppressor?}

One striking outcome of this project is the discrepancy when comparing our data to previously published reports. To date, several studies described that USP22 overexpression promotes tumor development or progression suggesting an oncogenic role of USP22. Most studies demonstrating elevated USP22 levels in cancer patient samples were based either on microarray data or immunohistochemical stainings. However, some of the antibodies used for the IHC approaches showed two or more bands when we tested them in western blot analyses. Thus, the specificity for USP22 of these antibodies is questionable and it would be possible that USP51 and/or USP27X which have a structure highly similar to USP22, were detected by these antibodies as well. As mentioned before, we could not find a commercially available antibody which specifically stains USP22 on tissue sections. Intriguingly, in the current study we could demonstrate in vivo and in vitro that USP22 loss can elevate tumor burden. Therefore, our results imply a rather tumor suppressive function of USP22. This is a novel finding which questions the universality of most previous studies focusing on the role of USP22 in cancer. Thus, we do not hypothesize that only one aspect can be true: that USP22 expression is either elevated or reduced in malignancies. Instead, it is well accepted that cancer reflects a highly heterogeneous group of diseases and the molecular signatures can be vastly diverse (Budinska et al., 2013). In our study, the heterogeneity of USP22 expression could be demonstrated in a number of experiments. First, when investigating USP22 expression levels in databases we observed that a high fraction of CRC patients were characterized by decreased USP22 expression levels. Second, heterozygous USP22 deletions were verified in approximately half of CRC patients. Accordingly, USP22 protein levels were shown to be highly heterogeneous in a panel of CRC cell lines and the knockdown of USP22 had diverse effects on the proliferation of these cells. Indeed, in most tested cell lines, USP22 ablation slowed the growth of CRC cells. However, some cell lines were not affected at all, while in HCT116 cells, proliferation was elevated upon the loss of USP22. It is challenging to find out which factors 
determine whether high or low USP22 levels promote tumorigenesis. In general, tumor biology is an extremely complex field since it can be influenced by several factors such as the site of the tumor, and therefore tissue-specific determinants, tumor subtype and genetic signature. Therefore, it is possible that USP22 loss only favors growth and progression of certain CRC subtypes. As mentioned in our results, Usp22- animals were characterized by the presence of mucus accumulations as well as tumors with a mucinous presentation. Moreover, we demonstrated that oncogenic growth was accelerated in an inflammatory environment when Usp22 levels were low. Therefore, this could mean that USP22 only acts as a tumor suppressor in cancer lesions with a mucinous appearance or under inflammatory circumstances while it otherwise functions as an oncogenic factor.

Moreover, it would be imaginable that the function of USP22 in tumorigenesis is indeed dependent on H2Bub1 levels. As mentioned before, H2Bub1 was described as a tumor suppressive mark (Cole et al., 2015; Melling et al., 2016) and under normal conditions, USP22 deubiquitinates the core histone H2B, thus, it mediates the decrease of H2Bub1 levels (Zhang et al., 2008b). Even though we could not observe any changes in H2Bub1 levels upon USP22 loss in our studies, depending on the tissue and tumor subtypes, USP22 could exert its cancerrelated effects via H2B deubiquitination. Thus, it would be possible that in some tissue types, overexpression of USP22 and the resultant decrease in H2Bub1 can be directly correlated to increased tumorigenesis. This could be the case in the previously published studies in which authors have described that increased USP22 expression leads to high oncogenic growth. However, counterintuitively, tumorigenesis could also be mediated by decreased H2Bub1 levels due to decreased HSP90 expression caused by USP22 ablation. This could occur if USP22 loss decreases HSP90 levels, PTEF-b complex assembly efficiency decreases and therefore the phosphorylation rates of the RNA Pol II are reduced. The same is true for BRD4 inhibition due to which PTEF-b can no longer be recruited by BRD4 and therefore RNA Pol II phosphorylation is impaired. Both scenarios can result in the condition that the adapter protein 
WAC is no longer recruited by RNA Pol II, the RNF20/RNF40 complex is not bound and therefore $\mathrm{H} 2 \mathrm{~B}$ is not ubiquitinated. Consequently, the loss of H2Bub1 could be correlated to increased tumor formation rates. In order to test these hypotheses, tissue microarrays generated from several cancer types could be tested for their H2Bub1 levels by IHC and for USP22 levels using an in situ hybridization-based approach. It would be of great value to determine whether H2Bub1 loss is specific to certain cancer subtypes and whether these findings correlate with USP22 levels.

Moreover, in future projects it would be interesting to elucidate whether USP22 can also act independently from the SAGA complex as has been demonstrated for USP51 and USP27X (Atanassov et al., 2016). To precipitate USP22 present in the SAGA complex, one could tag one SAGA-member and purify this protein including the USP22 bound to it. Using the same protein lysate the total USP22 amount can then be detected which allows the estimation of the abundance of "free", unbound USP22. Moreover, USP22 targets and interaction partners could be identified in a mass spectrometry-based approach. Whether USP22 functions via these targets could subsequently be determined by assessing the consequences after depleting these proteins. Together, while USP22 was described as an oncogene in literature, it displayed tumor suppressive properties in our experimental setup. However, further research is necessary to determine whether USP22 functions within or independent of the SAGA complex in tumorigenesis and whether this function is associated with $\mathrm{H} 2 \mathrm{~B}$ monoubiquitination.

\subsection{USP22 as a target in colorectal cancer therapy?}

As recently discussed in a review by Melo-Cardenas et al., based on the literature USP22 would represent an attractive target in cancer therapy. In fact, they reported that there is ongoing research to generate and optimize USP22 inhibitors (Melo-Cardenas et al., 2016). However, as shown in the current project, inhibiting USP22 could have devastating effects on 
colorectal cancer patients since it could even accelerate tumor growth and lead to the formation of invasive carcinomas. Therefore, it is essential to further elucidate the mechanisms underlying the aggressive oncogenic growth due to reduced USP22 levels. It would be important to know, for instance, if a certain tumor subtype such as mucinous carcinomas is linked to low USP22 levels. Using tumor biopsies, USP22 levels and tumor characteristics could be determined and, according to the outcome of these analyses, therapy options can be defined. With this approach it could be prevented that a protein is inhibited which, in fact, acts as a tumor suppressor in several patients. However, it would be challenging to unravel all aspects underlying the heterogeneity of USP22 expression in CRC and, therefore, even after patient stratification, there remains a risk of selecting a suboptimal therapy option. With this consideration in mind, it would be a safer option to exploit the fact that some CRC patients are characterized by low USP22 expression and treat them with drugs that are already approved due to their high tolerance and anti-tumor efficacy. In our project, we were able to successfully induce synthetic lethality by treating CRC cells with USP22 reduction either with Ganetespib or JQ1. Ganetespib, a small molecule inhibitor of HSP90, is a promising agent tested in a variety of cancer types (Proia and Bates, 2014). Moreover, its advantage is its low toxicity as, among others, shown in colorectal cancer patients (Cercek et al., 2014). Comparatively, BET inhibitors are well-tolerable and currently used in several clinical studies testing its efficacy in various types of malignancies and further diseases (Ferri et al., 2016). Therefore, after determining the genetic profile of CRC patients, treatment with Ganetespib or BET inhibitors could be promising in individuals with low USP22 expression levels. Moreover, as described before, the potency of a number of (epigenetic) inhibitors could be further improved by combining them with other therapeutics. For instance, combinations with BRAF and MEK inhibitors were shown to overcome drug resistance and enhance the efficacy of Ganetespib (Acquaviva et al., 2014). Similarly, in previous studies, synergistic effects could be observed after combining JQ1 with the mTOR inhibitor rapamycin (Lee et al., 2015) as well as histone 
deacetylase inhibitors (Shahbazi et al., 2016) and CDK inhibitors (Baker et al., 2015). Afterwards, it would be desirable to transplant our USP22-deficient CRISPR/Cas9 cells into immunodeficient mice via xenograft and test the effect of inhibition. Finally, we could create a new cohort of Usp22 $2^{\text {loxP }}$ Tam-DSS mice and directly treat them with the respective inhibitors after the knockout of Usp22. It would be particularly interesting from a clinical perspective if, indeed, the tumor burden in Usp22 $2^{--}$animals could be reduced or even eliminated by the inhibitor treatment.

\subsection{Concluding remarks}

To date, several studies identified a number of substrates deubiquitinated by USP22 and have reported USP22 overexpression in diverse cancer types. However, the exact physiological function of USP22 and the mechanisms underlying its involvement in oncogenic growth needed to be elucidated and were addressed in the current study. For this purpose, we performed in vivo, in vitro and in silico analyses. By analyzing genetic mouse models, consequences of a global reduction (Usp22 ${ }^{\text {lacz }}$ mice) and intestine-specific deletion (Usp22 ${ }^{\text {loxP }}$ mice) of Usp22 were detected in vivo, while human $\mathrm{CRC}$ cell lines and public databases were used for our in vitro and in silico studies, respectively.

By analyzing Usp22 $2^{\text {lacz }}$ animals we discovered that Usp22 is ubiquitously expressed throughout the majority of tissues in the developing embryo. The involvement of USP22 in developmental processes in vivo was demonstrated by the growth retardation in Usp22-hypomorphic mice. Moreover, differentiation and lineage specification in murine tissues are dependent on the presence of USP22. Finally, microarray analysis using MEFs underlined the relevance of USP22 during development and proliferation and confirmed our in vivo results. The most striking finding of this study was that USP22, in contrast to previously published reports, can also act as a tumor suppressor. When analyzing expression profiles of CRC patients using publically available databases, we could demonstrate that USP22 expression during colorectal 
carcinogenesis is not as 'black and white' as described in the literature. Indeed, almost half of CRC patients displayed decreased USP22 expression or even heterozygous deletions. Interestingly, our in vivo approaches suggest inflammation-promoting effects of Usp22 ablation. In fact, inflammation intensity upon Usp22 ablation was severe enough to induce colitis-induced bone fragility. Intriguingly, simultaneous loss of APC and USP22 resulted in decreased survival and increased intestinal tumor burden in sporadic and inflammationinduced CRC. Moreover, APC ${ }^{1638 N /+}$, Usp22 $2^{-/-}$mice even developed invasive carcinomas. Therefore, USP22 should not be exclusively considered as an oncogene since it can clearly function as a tumor suppressor, at least in some contexts. Importantly, the generation and application of USP22 inhibitors could display a high risk for CRC patients characterized by increased or normal USP22 expression since they could increase tumor burden by artificially causing decreased USP22 activity. In addition, these inhibitors could induce chronic inflammation. Instead, it would be a safe alternative to exploit USP22-deficiency in CRC cells by targeting HSP90AB1, a novel USP22 interaction partner identified in this project. The application of the HSP90 inhibitor Ganetespib as well as the epigenetic drug JQ1 induced synthetic lethality in CRC cells with low USP22 levels. In future experiments, the interaction of USP22 and HSP90AB1 should be studied in detail and the efficacy of Ganetespib and JQ1 tested in vivo. Taken together, in the current study we were able to demonstrate that USP22 is crucial for cell differentiation processes and has an unexpected tumor suppressor function in colorectal cancer. 


\section{REFERENCES}

Acquaviva, J., Smith, D.L., Jimenez, J.-P., Zhang, C., Sequeira, M., He, S., Sang, J., Bates, R.C., Proia, D.A., 2014. Overcoming acquired BRAF inhibitor resistance in melanoma via targeted inhibition of Hsp90 with ganetespib. Molecular cancer therapeutics 13 (2), 353363. 10.1158/1535-7163.MCT-13-0481.

Adams, S.M., Bornemann, P.H., 2013. Ulcerative colitis. American family physician 87 (10), 699-705.

Ahnen, D.J., 2011. The American College of Gastroenterology Emily Couric Lecture--the adenoma-carcinoma sequence revisited: has the era of genetic tailoring finally arrived? The American journal of gastroenterology 106 (2), 190-198. 10.1038/ajg.2010.423.

Akkoca, A.N., Yanik, S., Ozdemir, Z.T., Cihan, F.G., Sayar, S., Cincin, T.G., Cam, A., Ozer, C., 2014. TNM and Modified Dukes staging along with the demographic characteristics of patients with colorectal carcinoma. International journal of clinical and experimental medicine 7 (9), 2828-2835.

Ali, T., Lam, D., Bronze, M.S., Humphrey, M.B., 2009. Osteoporosis in inflammatory bowel disease. The American journal of medicine 122 (7), 599-604. 10.1016/j.amjmed.2009.01.022.

Ao, N., Liu, Y., Bian, X., Feng, H., Liu, Y., 2015. Ubiquitin-specific peptidase 22 inhibits colon cancer cell invasion by suppressing the signal transducer and activator of transcription 3/matrix metalloproteinase 9 pathway. Molecular medicine reports 12 (2), 2107-2113. 10.3892/mmr.2015.3661.

Ao, N., Liu, Y., Feng, H., Bian, X., Li, Z., Gu, B., Zhao, X., Liu, Y., 2014. Ubiquitin-specific peptidase USP22 negatively regulates the STAT signaling pathway by deubiquitinating SIRT1. Cellular physiology and biochemistry : international journal of experimental cellular physiology, biochemistry, and pharmacology 33 (6), 1863-1875. 10.1159/000362964.

Arslan, G., Atasever, T., Cindoruk, M., Yildirim, I.S., 2001. (51)CrEDTA colonic permeability and therapy response in patients with ulcerative colitis. Nuclear medicine communications $22(9), 997-1001$.

Aschele, C., Cionini, L., Lonardi, S., Pinto, C., Cordio, S., Rosati, G., Artale, S., Tagliagambe, A., Ambrosini, G., Rosetti, P., Bonetti, A., Negru, M.E., Tronconi, M.C., Luppi, G., Silvano, G., Corsi, D.C., Bochicchio, A.M., Chiaulon, G., Gallo, M., Boni, L., 2011. Primary tumor response to preoperative chemoradiation with or without oxaliplatin in locally advanced rectal cancer: pathologic results of the STAR-01 randomized phase III trial. Journal of clinical oncology : official journal of the American Society of Clinical Oncology 29 (20), 2773-2780. 10.1200/JCO.2010.34.4911.

Atanassov, B.S., Evrard, Y.A., Multani, A.S., Zhang, Z., Tora, L., Devys, D., Chang, S., Dent, S.Y.R., 2009. Gcn5 and SAGA regulate shelterin protein turnover and telomere maintenance. Molecular cell 35 (3), 352-364. 10.1016/j.molcel.2009.06.015. 
Atanassov, B.S., Mohan, R.D., Lan, X., Kuang, X., Lu, Y., Lin, K., Mclvor, E., Li, W., Zhang, Y., Florens, L., Byrum, S.D., Mackintosh, S.G., Calhoun-Davis, T., Koutelou, E., Wang, L., Tang, D.G., Tackett, A.J., Washburn, M.P., Workman, J.L., Dent, S.Y.R., 2016. ATXN7L3 and ENY2 Coordinate Activity of Multiple H2B Deubiquitinases Important for Cellular Proliferation and Tumor Growth. Molecular cell 62 (4), 558-571. 10.1016/j.molcel.2016.03.030.

Aune, D., Lau, R., Chan, D.S.M., Vieira, R., Greenwood, D.C., Kampman, E., Norat, T., 2012. Dairy products and colorectal cancer risk: a systematic review and meta-analysis of cohort studies. Annals of oncology : official journal of the European Society for Medical Oncology 23 (1), 37-45. 10.1093/annonc/mdr269.

Bacher, J.W., Abdel Megid, W.M., Kent-First, M.G., Halberg, R.B., 2005. Use of mononucleotide repeat markers for detection of microsatellite instability in mouse tumors. Molecular carcinogenesis 44 (4), 285-292. 10.1002/mc.20146.

Baena, R., Salinas, P., 2015. Diet and colorectal cancer. Maturitas 80 (3), 258-264. 10.1016/j.maturitas.2014.12.017.

Bailie, L., Loughrey, M.B., Coleman, H.G., 2016. Lifestyle Risk Factors for Serrated Colorectal Polyps: A Systematic Review and Meta-Analysis. Gastroenterology. 10.1053/j.gastro.2016.09.003.

Baker, E.K., Taylor, S., Gupte, A., Sharp, P.P., Walia, M., Walsh, N.C., Zannettino, A.C.W., Chalk, A.M., Burns, C.J., Walkley, C.R., 2015. BET inhibitors induce apoptosis through a MYC independent mechanism and synergise with CDK inhibitors to kill osteosarcoma cells. Scientific reports 5, 10120. 10.1038/srep10120.

Baker, S.P., Grant, P.A., 2007. The SAGA continues: expanding the cellular role of a transcriptional co-activator complex. Oncogene 26 (37), 5329-5340. 10.1038/sj.onc. 1210603 .

Barker, N., Ridgway, R.A., van Es, J.H., van de Wetering, M., Begthel, H., van den Born, M., Danenberg, E., Clarke, A.R., Sansom, O.J., Clevers, H., 2009. Crypt stem cells as the cells-of-origin of intestinal cancer. Nature 457 (7229), 608-611. 10.1038/nature07602.

Barker, N., van Es, J.H., Kuipers, J., Kujala, P., van den Born, M., Cozijnsen, M., Haegebarth, A., Korving, J., Begthel, H., Peters, P.J., Clevers, H., 2007. Identification of stem cells in small intestine and colon by marker gene Lgr5. Nature 449 (7165), 10031007. 10.1038/nature06196.

Barker, N., van Oudenaarden, A., Clevers, H., 2012. Identifying the stem cell of the intestinal crypt: strategies and pitfalls. Cell stem cell 11 (4), 452-460. 10.1016/j.stem.2012.09.009.

Barmeyer, C., Schulzke, J.D., Fromm, M., 2015. Claudin-related intestinal diseases. Seminars in cell \& developmental biology 42, 30-38. 10.1016/j.semcdb.2015.05.006.

Baumgart, D.C., Sandborn, W.J., 2007. Inflammatory bowel disease: clinical aspects and established and evolving therapies. Lancet (London, England) 369 (9573), 1641-1657. 10.1016/S0140-6736(07)60751-X. 
Bedi, U., Scheel, A.H., Hennion, M., Begus-Nahrmann, Y., Ruschoff, J., Johnsen, S.A., 2015. SUPT6H controls estrogen receptor activity and cellular differentiation by multiple epigenomic mechanisms. Oncogene 34 (4), 465-473. 10.1038/onc.2013.558.

Ben, Q., Sun, Y., Chai, R., Qian, A., Xu, B., Yuan, Y., 2014. Dietary fiber intake reduces risk for colorectal adenoma: a meta-analysis. Gastroenterology 146 (3), 689-699.e6. 10.1053/j.gastro.2013.11.003.

Bernstein, C.N., Blanchard, J.F., Leslie, W., Wajda, A., Yu, B.N., 2000. The incidence of fracture among patients with inflammatory bowel disease. A population-based cohort study. Annals of internal medicine 133 (10), 795-799.

Bernstein, C.N., Fried, M., Krabshuis, J.H., Cohen, H., Eliakim, R., Fedail, S., Gearry, R., Goh, K.L., Hamid, S., Khan, A.G., LeMair, A.W., Malfertheiner, Ouyang, Q., Rey, J.F., Sood, A., Steinwurz, F., Thomsen, O.O., Thomson, A., Watermeyer, G., 2010. World Gastroenterology Organization Practice Guidelines for the diagnosis and management of IBD in 2010. Inflammatory bowel diseases 16 (1), 112-124. 10.1002/ibd.21048.

Berry, R.J., 1900. The True Caecal Apex, or the Vermiform Appendix: Its Minute and Comparative Anatomy. Journal of anatomy and physiology 35 (Pt 1), 83-100.9.

Block, G., Patterson, B., Subar, A., 1992. Fruit, vegetables, and cancer prevention: a review of the epidemiological evidence. Nutrition and cancer 18 (1), 1-29. $10.1080 / 01635589209514201$.

Boettiger, A.N., Ralph, P.L., Evans, S.N., 2011. Transcriptional regulation: effects of promoter proximal pausing on speed, synchrony and reliability. PLoS computational biology 7 (5), e1001136. 10.1371/journal.pcbi.1001136.

Bose, P., Dai, Y., Grant, S., 2014. Histone deacetylase inhibitor (HDACI) mechanisms of action: emerging insights. Pharmacology \& therapeutics 143 (3), 323-336. 10.1016/j.pharmthera.2014.04.004.

Bose, P., Simmons, G.L., Grant, S., 2013. Cyclin-dependent kinase inhibitor therapy for hematologic malignancies. Expert opinion on investigational drugs 22 (6), 723-738. 10.1517/13543784.2013.789859.

Bosetti, C., Levi, F., Rosato, V., Bertuccio, P., Lucchini, F., Negri, E., La Vecchia, C., 2011. Recent trends in colorectal cancer mortality in Europe. International journal of cancer 129 (1), 180-191. 10.1002/ijc.25653.

Boyle, P., Ferlay, J., 2005. Mortality and survival in breast and colorectal cancer. Nature clinical practice. Oncology 2 (9), 424-425. 10.1038/ncponc0288.

Brenner, D.J., 2004. Radiation risks potentially associated with low-dose CT screening of adult smokers for lung cancer. Radiology 231 (2), 440-445. 10.1148/radiol.2312030880.

Budinska, E., Popovici, V., Tejpar, S., D'Ario, G., Lapique, N., Sikora, K.O., Di Narzo, A.F., Yan, P., Hodgson, J.G., Weinrich, S., Bosman, F., Roth, A., Delorenzi, M., 2013. Gene expression patterns unveil a new level of molecular heterogeneity in colorectal cancer. The Journal of pathology 231 (1), 63-76. 10.1002/path.4212. 
Burns, R., Leal, J., Sullivan, R., Luengo-Fernandez, R., 2016. Economic burden of malignant blood disorders across Europe: a population-based cost analysis. The Lancet. Haematology 3 (8), e362-70. 10.1016/S2352-3026(16)30062-X.

Canny, G.O., McCormick, B.A., 2008. Bacteria in the intestine, helpful residents or enemies from within? Infection and immunity 76 (8), 3360-3373. 10.1128/IAI.00187-08.

Carre, C., Szymczak, D., Pidoux, J., Antoniewski, C., 2005. The histone H3 acetylase dGcn5 is a key player in Drosophila melanogaster metamorphosis. Molecular and cellular biology 25 (18), 8228-8238. 10.1128/MCB.25.18.8228-8238.2005.

Cerami, E., Gao, J., Dogrusoz, U., Gross, B.E., Sumer, S.O., Aksoy, B.A., Jacobsen, A., Byrne, C.J., Heuer, M.L., Larsson, E., Antipin, Y., Reva, B., Goldberg, A.P., Sander, C., Schultz, N., 2012. The cBio cancer genomics portal: an open platform for exploring multidimensional cancer genomics data. Cancer discovery 2 (5), 401-404. 10.1158/21598290.CD-12-0095.

Cercek, A., Shia, J., Gollub, M., Chou, J.F., Capanu, M., Raasch, P., Reidy-Lagunes, D., Proia, D.A., Vakiani, E., Solit, D.B., Saltz, L.B., 2014. Ganetespib, a novel Hsp90 inhibitor in patients with KRAS mutated and wild type, refractory metastatic colorectal cancer. Clinical colorectal cancer 13 (4), 207-212. 10.1016/j.clcc.2014.09.001.

Chan, D.S.M., Lau, R., Aune, D., Vieira, R., Greenwood, D.C., Kampman, E., Norat, T., 2011. Red and processed meat and colorectal cancer incidence: meta-analysis of prospective studies. PloS one 6 (6), e20456. 10.1371/journal.pone.0020456.

Chang, F., Steelman, L.S., Lee, J.T., Shelton, J.G., Navolanic, P.M., Blalock, W.L., Franklin, R.A., McCubrey, J.A., 2003. Signal transduction mediated by the Ras/Raf/MEK/ERK pathway from cytokine receptors to transcription factors: potential targeting for therapeutic intervention. Leukemia 17 (7), 1263-1293. 10.1038/sj.leu.2402945.

Chassaing, B., Aitken, J.D., Malleshappa, M., Vijay-Kumar, M., 2014. Dextran sulfate sodium (DSS)-induced colitis in mice. Current protocols in immunology 104, Unit 15.25. 10.1002/0471142735.im1525s104.

Choi, M., Lim, S., Choi, M.-G., Shim, K.-N., Lee, S.H., 2016. Effectiveness of Capsule Endoscopy Compared with Other Diagnostic Modalities in Patients with Small Bowel Crohn's Disease: A Meta-Analysis. Gut and liver. 10.5009/gnl16015.

Clayburgh, D.R., Le Shen, Turner, J.R., 2004. A porous defense: the leaky epithelial barrier in intestinal disease. Laboratory investigation; a journal of technical methods and pathology 84 (3), 282-291. 10.1038/labinvest.3700050.

Clevers, H.C., Bevins, C.L., 2013. Paneth cells: maestros of the small intestinal crypts. Annual review of physiology 75, 289-311. 10.1146/annurev-physiol-030212-183744.

Cole, A.J., Clifton-Bligh, R., Marsh, D.J., 2015. Histone H2B monoubiquitination: roles to play in human malignancy. Endocrine-related cancer 22 (1), T19-33. 10.1530/ERC-14-0185.

Colland, F., Formstecher, E., Jacq, X., Reverdy, C., Planquette, C., Conrath, S., Trouplin, V., Bianchi, J., Aushev, V.N., Camonis, J., Calabrese, A., Borg-Capra, C., Sippl, W., Collura, 
V., Boissy, G., Rain, J.-C., Guedat, P., Delansorne, R., Daviet, L., 2009. Small-molecule inhibitor of USP7/HAUSP ubiquitin protease stabilizes and activates p53 in cells. Molecular cancer therapeutics 8 (8), 2286-2295. 10.1158/1535-7163.MCT-09-0097.

Colman, R.J., Rubin, D.T., 2014. Fecal microbiota transplantation as therapy for inflammatory bowel disease: a systematic review and meta-analysis. Journal of Crohn's \& colitis 8 (12), 1569-1581. 10.1016/j.crohns.2014.08.006.

Comito, D., Cascio, A., Romano, C., 2014. Microbiota biodiversity in inflammatory bowel disease. Italian journal of pediatrics 40, 32. 10.1186/1824-7288-40-32.

Coppede, F., Lopomo, A., Spisni, R., Migliore, L., 2014. Genetic and epigenetic biomarkers for diagnosis, prognosis and treatment of colorectal cancer. World journal of gastroenterology 20 (4), 943-956. 10.3748/wjg.v20.i4.943.

Costello, J.F., Fruhwald, M.C., Smiraglia, D.J., Rush, L.J., Robertson, G.P., Gao, X., Wright, F.A., Feramisco, J.D., Peltomaki, P., Lang, J.C., Schuller, D.E., Yu, L., Bloomfield, C.D., Caligiuri, M.A., Yates, A., Nishikawa, R., Su Huang, H., Petrelli, N.J., Zhang, X., O'Dorisio, M.S., Held, W.A., Cavenee, W.K., Plass, C., 2000. Aberrant CpG-island methylation has non-random and tumour-type-specific patterns. Nature genetics 24 (2), 132-138. 10.1038/72785.

Courtney, K.D., Corcoran, R.B., Engelman, J.A., 2010. The PI3K pathway as drug target in human cancer. Journal of clinical oncology : official journal of the American Society of Clinical Oncology 28 (6), 1075-1083. 10.1200/JCO.2009.25.3641.

Cremers, N., Neeb, A., Uhle, T., Dimmler, A., Rothley, M., Allgayer, H., Fodde, R., Sleeman, J.P., Thiele, W., 2016. CD24 Is Not Required for Tumor Initiation and Growth in Murine Breast and Prostate Cancer Models. PloS one 11 (3), e0151468. 10.1371/journal.pone.0151468.

Cummins, J.M., Rago, C., Kohli, M., Kinzler, K.W., Lengauer, C., Vogelstein, B., 2004. Tumour suppression: disruption of HAUSP gene stabilizes p53. Nature 428 (6982), 1 p following 486. 10.1038/nature02501.

Cunningham, D., Atkin, W., Lenz, H.-J., Lynch, H.T., Minsky, B., Nordlinger, B., Starling, N., 2010. Colorectal cancer. Lancet (London, England) 375 (9719), 1030-1047. 10.1016/S0140-6736(10)60353-4.

Dantzer, F., La Rubia, G. de, Menissier-De Murcia, J., Hostomsky, Z., Murcia, G. de, Schreiber, V., 2000. Base excision repair is impaired in mammalian cells lacking Poly(ADP-ribose) polymerase-1. Biochemistry 39 (25), 7559-7569.

David, L.A., Maurice, C.F., Carmody, R.N., Gootenberg, D.B., Button, J.E., Wolfe, B.E., Ling, A.V., Devlin, A.S., Varma, Y., Fischbach, M.A., Biddinger, S.B., Dutton, R.J., Turnbaugh, P.J., 2014. Diet rapidly and reproducibly alters the human gut microbiome. Nature 505 (7484), 559-563. 10.1038/nature12820.

Davidovich, P., Kearney, C.J., Martin, S.J., 2014. Inflammatory outcomes of apoptosis, necrosis and necroptosis. Biological chemistry 395 (10), 1163-1171. 10.1515/hsz-20140164. 
Dawood, S., Austin, L., Cristofanilli, M., 2014. Cancer stem cells: implications for cancer therapy. Oncology (Williston Park, N.Y.) 28 (12), 1101-7, 1110.

Despopoulos, A., Silbernagl, S., 2003. Color atlas of physiology, 5th ed. Thieme, Stuttgart, $436 \mathrm{pp}$.

Dexheimer, T.S., Rosenthal, A.S., Liang, Q., Chen, J., Villamil, M.A., Kerns, E.H., Simeonov, A., Jadhav, A., Zhuang, Z., Maloney, D.J., 2010. Discovery of ML323 as a Novel Inhibitor of the USP1/UAF1 Deubiquitinase Complex, in: Probe Reports from the NIH Molecular Libraries Program, Bethesda (MD).

Dimitriou, N., Griniatsos, J., 2015. Complete mesocolic excision: Techniques and outcomes. World journal of gastrointestinal oncology 7 (12), 383-388. 10.4251/wjgo.v7.i12.383.

Ding, F., Bao, C., Tian, Y., Xiao, H., Wang, M., Xie, X., Hu, F., Mei, J., 2014. USP22 promotes NSCLC tumorigenesis via MDMX up-regulation and subsequent p53 inhibition. International journal of molecular sciences 16 (1), 307-320. 10.3390/ijms16010307.

Dollive, S., Peterfreund, G.L., Sherrill-Mix, S., Bittinger, K., Sinha, R., Hoffmann, C., Nabel, C.S., Hill, D.A., Artis, D., Bachman, M.A., Custers-Allen, R., Grunberg, S., Wu, G.D., Lewis, J.D., Bushman, F.D., 2012. A tool kit for quantifying eukaryotic rRNA gene sequences from human microbiome samples. Genome biology 13 (7), R60. 10.1186/gb2012-13-7-r60.

Donovan, A., Lima, C.A., Pinkus, J.L., Pinkus, G.S., Zon, L.I., Robine, S., Andrews, N.C., 2005. The iron exporter ferroportin/Slc40a1 is essential for iron homeostasis. Cell metabolism 1 (3), 191-200. 10.1016/j.cmet.2005.01.003.

Duan, Y., Huo, D., Gao, J., Wu, H., Ye, Z., Liu, Z., Zhang, K., Shan, L., Zhou, X., Wang, Y., Su, D., Ding, X., Shi, L., Wang, Y., Shang, Y., Xuan, C., 2016. Ubiquitin ligase RNF20/40 facilitates spindle assembly and promotes breast carcinogenesis through stabilizing motor protein Eg5. Nature communications 7, 12648. 10.1038/ncomms12648.

Eccles, D.M., Lunt, P.W., Wallis, Y., Griffiths, M., Sandhu, B., McKay, S., Morton, D., SheaSimonds, J., Macdonald, F., 1997. An unusually severe phenotype for familial adenomatous polyposis. Archives of disease in childhood 77 (5), 431-435.

el Marjou, F., Janssen, K.-P., Chang, B.H.-J., Li, M., Hindie, V., Chan, L., Louvard, D., Chambon, P., Metzger, D., Robine, S., 2004. Tissue-specific and inducible Cre-mediated recombination in the gut epithelium. Genesis (New York, N.Y. : 2000) 39 (3), 186-193. 10.1002/gene.20042.

El Zoghbi, M., Cummings, L.C., 2016. New era of colorectal cancer screening. World journal of gastrointestinal endoscopy 8 (5), 252-258. 10.4253/wjge.v8.i5.252.

Ellis, H., 2006. Clinical anatomy: A revision and applied anatomy for clinical students, 11th ed. Blackwell Science, Oxford, xiv, 439.

Engelman, J.A., Luo, J., Cantley, L.C., 2006. The evolution of phosphatidylinositol 3-kinases as regulators of growth and metabolism. Nature reviews. Genetics 7 (8), 606-619. 10.1038/nrg1879. 
Falcone, A., Ricci, S., Brunetti, I., Pfanner, E., Allegrini, G., Barbara, C., Crino, L., Benedetti, G., Evangelista, W., Fanchini, L., Cortesi, E., Picone, V., Vitello, S., Chiara, S., Granetto, C., Porcile, G., Fioretto, L., Orlandini, C., Andreuccetti, M., Masi, G., 2007. Phase III trial of infusional fluorouracil, leucovorin, oxaliplatin, and irinotecan (FOLFOXIRI) compared with infusional fluorouracil, leucovorin, and irinotecan (FOLFIRI) as first-line treatment for metastatic colorectal cancer: the Gruppo Oncologico Nord Ovest. Journal of clinical oncology : official journal of the American Society of Clinical Oncology 25 (13), 16701676. 10.1200/JCO.2006.09.0928.

Fan, Y.-H., Cheng, J., Vasudevan, S.A., Dou, J., Zhang, H., Patel, R.H., Ma, I.T., Rojas, Y., Zhao, Y., Yu, Y., Shohet, J.M., Nuchtern, J.G., Kim, E.S., Yang, J., 2013. USP7 inhibitor P22077 inhibits neuroblastoma growth via inducing p53-mediated apoptosis. Cell death \& disease 4, e867. 10.1038/cddis.2013.400.

Fang, D., Hawke, D., Zheng, Y., Xia, Y., Meisenhelder, J., Nika, H., Mills, G.B., Kobayashi, R., Hunter, T., Lu, Z., 2007. Phosphorylation of beta-catenin by AKT promotes betacatenin transcriptional activity. The Journal of biological chemistry 282 (15), 1122111229. 10.1074/jbc.M611871200.

Favoriti, P., Carbone, G., Greco, M., Pirozzi, F., Pirozzi, R.E.M., Corcione, F., 2016. Worldwide burden of colorectal cancer: a review. Updates in surgery 68 (1), 7-11. 10.1007/s13304-016-0359-y.

Fearon, E.R., 2011. Molecular genetics of colorectal cancer. Annual review of pathology 6, 479-507. 10.1146/annurev-pathol-011110-130235.

Fearon, E.R., Vogelstein, B., 1990. A genetic model for colorectal tumorigenesis. Cell 61 (5), 759-767.

Feil, S., Valtcheva, N., Feil, R., 2009. Inducible Cre mice. Methods in molecular biology (Clifton, N.J.) 530, 343-363. 10.1007/978-1-59745-471-1_18.

Feinberg, A.P., Tycko, B., 2004. The history of cancer epigenetics. Nature reviews. Cancer 4 (2), 143-153. 10.1038/nrc1279.

Feinberg, A.P., Vogelstein, B., 1983. Hypomethylation distinguishes genes of some human cancers from their normal counterparts. Nature 301 (5895), 89-92.

Felekis, T., Katsanos, K., Kitsanou, M., Trakos, N., Theopistos, V., Christodoulou, D., Asproudis, I., Tsianos, E.V., 2009. Spectrum and frequency of ophthalmologic manifestations in patients with inflammatory bowel disease: a prospective single-center study. Inflammatory bowel diseases 15 (1), 29-34. 10.1002/ibd.20584.

Feng, Y., Sentani, K., Wiese, A., Sands, E., Green, M., Bommer, G.T., Cho, K.R., Fearon, E.R., 2013. Sox9 induction, ectopic Paneth cells, and mitotic spindle axis defects in mouse colon adenomatous epithelium arising from conditional biallelic Apc inactivation. The American journal of pathology 183 (2), 493-503. 10.1016/j.ajpath.2013.04.013.

Ferlay, 2016. Cancer preventability estimates: ESTIMATED CASES OF US CANCERS PREVENTABLE PER YEAR BY DIET, ACTIVITY, AND WEIGHT MANAGEMENT. 
http://www.aicr.org/research/research_science_policy_report.html. Accessed 11 December 2016.

Ferri, E., Petosa, C., McKenna, C.E., 2016. Bromodomains: Structure, function and pharmacology of inhibition. Biochemical pharmacology 106, 1-18. 10.1016/j.bcp.2015.12.005.

Fleming, M., Ravula, S., Tatishchev, S.F., Wang, H.L., 2012. Colorectal carcinoma: Pathologic aspects. Journal of gastrointestinal oncology 3 (3), 153-173. 10.3978/j.issn.2078-6891.2012.030.

Fodde, R., Edelmann, W., Yang, K., van Leeuwen, C., Carlson, C., Renault, B., Breukel, C., Alt, E., Lipkin, M., Khan, P.M., 1994. A targeted chain-termination mutation in the mouse Apc gene results in multiple intestinal tumors. Proceedings of the National Academy of Sciences of the United States of America 91 (19), 8969-8973.

Fodde, R., Smits, R., 2001. Disease model: familial adenomatous polyposis. Trends in molecular medicine 7 (8), 369-373.

Frick, J.-S., Autenrieth, I.B., 2013. The gut microflora and its variety of roles in health and disease. Current topics in microbiology and immunology 358, 273-289. 10.1007/82_2012_217.

Friedel, D., Modayil, R., Stavropoulos, S., 2016. Colon Capsule Endoscopy: Review and Perspectives. Gastroenterology research and practice 2016, 9643162. $10.1155 / 2016 / 9643162$.

Fuchs, C.S., Marshall, J., Mitchell, E., Wierzbicki, R., Ganju, V., Jeffery, M., Schulz, J., Richards, D., Soufi-Mahjoubi, R., Wang, B., Barrueco, J., 2007. Randomized, controlled trial of irinotecan plus infusional, bolus, or oral fluoropyrimidines in first-line treatment of metastatic colorectal cancer: results from the BICC-C Study. Journal of clinical oncology : official journal of the American Society of Clinical Oncology 25 (30), 4779-4786. 10.1200/JCO.2007.11.3357.

Fukata, N., Uchida, K., Kusuda, T., Koyabu, M., Miyoshi, H., Fukui, T., Matsushita, M., Nishio, A., Tabata, Y., Okazaki, K., 2011. The effective therapy of cyclosporine A with drug delivery system in experimental colitis. Journal of drug targeting 19 (6), 458-467. $10.3109 / 1061186 \times .2010 .511224$.

Galeone, C., Pelucchi, C., La Vecchia, C., 2012. Added sugar, glycemic index and load in colon cancer risk. Current opinion in clinical nutrition and metabolic care 15 (4), 368-373. 10.1097/MCO.0b013e3283539f81.

Gao, J., Aksoy, B.A., Dogrusoz, U., Dresdner, G., Gross, B., Sumer, S.O., Sun, Y., Jacobsen, A., Sinha, R., Larsson, E., Cerami, E., Sander, C., Schultz, N., 2013. Integrative analysis of complex cancer genomics and clinical profiles using the cBioPortal. Science signaling 6 (269), pl1. 10.1126/scisignal.2004088.

Gao, Y., Lin, F., Xu, P., Nie, J., Chen, Z., Su, J., Tang, J., Wu, Q., Li, Y., Guo, Z., Gao, Z., Li, D., Shen, J., Ge, S., Tsun, A., Li, B., 2014. USP22 is a positive regulator of NFATc2 on promoting IL2 expression. FEBS letters 588 (6), 878-883. 10.1016/j.febslet.2014.02.016. 
Garofalo, A., Chirivi, R.G., Scanziani, E., Mayo, J.G., Vecchi, A., Giavazzi, R., 1993. Comparative study on the metastatic behavior of human tumors in nude, beige/nude/xid and severe combined immunodeficient mice. Invasion \& metastasis 13 (2), 82-91.

Gartner, L.P., Hiatt, J.L., 2014. Color atlas and text of histology, 6th ed. Wolters Kluwer Health/Lippincott Williams \& Wilkins, Philadelphia, 525 pp.

Ghoshal, K., Datta, J., Majumder, S., Bai, S., Kutay, H., Motiwala, T., Jacob, S.T., 2005. 5Aza-deoxycytidine induces selective degradation of DNA methyltransferase 1 by a proteasomal pathway that requires the KEN box, bromo-adjacent homology domain, and nuclear localization signal. Molecular and cellular biology 25 (11), 4727-4741. 10.1128/MCB.25.11.4727-4741.2005.

Giles, R.H., van Es, J.H., Clevers, H., 2003. Caught up in a Wnt storm: Wnt signaling in cancer. Biochimica et biophysica acta 1653 (1), 1-24.

Glinsky, G.V., 2005. Death-from-cancer signatures and stem cell contribution to metastatic cancer. Cell cycle (Georgetown, Tex.) 4 (9), 1171-1175. 10.4161/cc.4.9.2001.

Glinsky, G.V., 2006. Genomic models of metastatic cancer: functional analysis of death-fromcancer signature genes reveals aneuploid, anoikis-resistant, metastasis-enabling phenotype with altered cell cycle control and activated Polycomb Group (PcG) protein chromatin silencing pathway. Cell cycle (Georgetown, Tex.) 5 (11), 1208-1216. 10.4161/cc.5.11.2796.

Glinsky, G.V., Berezovska, O., Glinskii, A.B., 2005. Microarray analysis identifies a deathfrom-cancer signature predicting therapy failure in patients with multiple types of cancer. The Journal of clinical investigation 115 (6), 1503-1521. 10.1172/JCI23412.

Godos, J., Bella, F., Torrisi, A., Sciacca, S., Galvano, F., Grosso, G., 2016. Dietary patterns and risk of colorectal adenoma: a systematic review and meta-analysis of observational studies. Journal of human nutrition and dietetics : the official journal of the British Dietetic Association 29 (6), 757-767. 10.1111/jhn.12395.

Gramont, A. de, Bosset, J.F., Milan, C., Rougier, P., Bouche, O., Etienne, P.L., Morvan, F., Louvet, C., Guillot, T., Francois, E., Bedenne, L., 1997. Randomized trial comparing monthly low-dose leucovorin and fluorouracil bolus with bimonthly high-dose leucovorin and fluorouracil bolus plus continuous infusion for advanced colorectal cancer: a French intergroup study. Journal of clinical oncology : official journal of the American Society of Clinical Oncology 15 (2), 808-815. 10.1200/jco.1997.15.2.808.

Graser, A., Melzer, A., Lindner, E., Nagel, D., Herrmann, K., Stieber, P., Schirra, J., Mansmann, U., Reiser, M.F., Goke, B., Kolligs, F.T., 2013. Magnetic resonance colonography for the detection of colorectal neoplasia in asymptomatic adults. Gastroenterology 144 (4), 743-750.e2. 10.1053/j.gastro.2012.12.041.

Guariso, G., Gasparetto, M., Visona Dalla Pozza, L., D'Inca, R., Zancan, L., Sturniolo, G., Brotto, F., Facchin, P., 2010. Inflammatory bowel disease developing in paediatric and adult age. Journal of pediatric gastroenterology and nutrition 51 (6), 698-707. 10.1097/MPG.0b013e3181da1db8. 
Guenther, M.G., Levine, S.S., Boyer, L.A., Jaenisch, R., Young, R.A., 2007. A chromatin landmark and transcription initiation at most promoters in human cells. Cell 130 (1), 7788. 10.1016/j.cell.2007.05.042.

Guinney, J., Dienstmann, R., Wang, X., Reynies, A. de, Schlicker, A., Soneson, C., Marisa, L., Roepman, P., Nyamundanda, G., Angelino, P., Bot, B.M., Morris, J.S., Simon, I.M., Gerster, S., Fessler, E., De Sousa E Melo, Felipe, Missiaglia, E., Ramay, H., Barras, D., Homicsko, K., Maru, D., Manyam, G.C., Broom, B., Boige, V., Perez-Villamil, B., Laderas, T., Salazar, R., Gray, J.W., Hanahan, D., Tabernero, J., Bernards, R., Friend, S.H., Laurent-Puig, P., Medema, J.P., Sadanandam, A., Wessels, L., Delorenzi, M., Kopetz, S., Vermeulen, L., Tejpar, S., 2015. The consensus molecular subtypes of colorectal cancer. Nature medicine 21 (11), 1350-1356. 10.1038/nm.3967.

Guraya, S.Y., 2015. Association of type 2 diabetes mellitus and the risk of colorectal cancer: A meta-analysis and systematic review. World journal of gastroenterology 21 (19), 60266031. 10.3748/wjg.v21.i19.6026.

Gurskii, D.I., Kopytova, D.V., Georgieva, S.G., Nabirochkina, E.N., 2013. SAGA complex: the role in viability and development. Molekuliarnaia biologiia 47 (6), 922-926.

Hafner, M.F., Debus, J., 2016. Radiotherapy for Colorectal Cancer: Current Standards and Future Perspectives. Visceral medicine 32 (3), 172-177. 10.1159/000446486.

Hallberg, B., Rayter, S.I., Downward, J., 1994. Interaction of Ras and Raf in intact mammalian cells upon extracellular stimulation. The Journal of biological chemistry 269 (6), 3913-3916.

Hamilton S.R., A.L., 2000. World Health Organization Classification of Tumours.: Pathology and Genetics of Tumours of the Digestive System.

Hampel, H., Frankel, W.L., Martin, E., Arnold, M., Khanduja, K., Kuebler, P., Clendenning, M., Sotamaa, K., Prior, T., Westman, J.A., Panescu, J., Fix, D., Lockman, J., LaJeunesse, J., Comeras, I., La Chapelle, A. de, 2008. Feasibility of screening for Lynch syndrome among patients with colorectal cancer. Journal of clinical oncology : official journal of the American Society of Clinical Oncology 26 (35), 5783-5788. 10.1200/JCO.2008.17.5950.

Hatch, Q., Steele, S.R., 2013. Rectal prolapse and intussusception. Gastroenterology clinics of North America 42 (4), 837-861. 10.1016/j.gtc.2013.08.002.

Hemminki, K., Santi, I., Weires, M., Thomsen, H., Sundquist, J., Bermejo, J.L., 2010. Tumor location and patient characteristics of colon and rectal adenocarcinomas in relation to survival and TNM classes. BMC cancer 10, 688. 10.1186/1471-2407-10-688.

Herman, J.G., Baylin, S.B., 2003. Gene silencing in cancer in association with promoter hypermethylation. The New England journal of medicine 349 (21), 2042-2054. 10.1056/NEJMra023075.

Higashi, D., Futami, K., Ishibashi, Y., Egawa, Y., Maekawa, T., Matsui, T., Iwashita, A., Kuroki, M., 2011. Clinical course of colorectal cancer in patients with ulcerative colitis. Anticancer research 31 (7), 2499-2504. 
Hinoi, T., Tani, M., Lucas, P.C., Caca, K., Dunn, R.L., Macri, E., Loda, M., Appelman, H.D., Cho, K.R., Fearon, E.R., 2001. Loss of CDX2 expression and microsatellite instability are prominent features of large cell minimally differentiated carcinomas of the colon. The American journal of pathology 159 (6), 2239-2248. 10.1016/S0002-9440(10)63074-X.

Hoffman, R.M., 1999. Orthotopic metastatic mouse models for anticancer drug discovery and evaluation: a bridge to the clinic. Investigational new drugs 17 (4), 343-359.

Hollander, D., Vadheim, C.M., Brettholz, E., Petersen, G.M., Delahunty, T., Rotter, J.I., 1986. Increased intestinal permeability in patients with Crohn's disease and their relatives. A possible etiologic factor. Annals of internal medicine 105 (6), 883-885.

Honjo, H., Mike, M., Kusanagi, H., Kano, N., 2015. Adult intussusception: a retrospective review. World journal of surgery 39 (1), 134-138. 10.1007/s00268-014-2759-9.

Hooper, L.V., Midtvedt, T., Gordon, J.I., 2002. How host-microbial interactions shape the nutrient environment of the mammalian intestine. Annual review of nutrition 22, 283-307. 10.1146/annurev.nutr.22.011602.092259.

Hossan, T., Nagarajan, S., Baumgart, S.J., Xie, W., Magallanes, R.T., Hernandez, C., Chiaroni, P.-M., Indenbirken, D., Spitzner, M., Thomas-Chollier, M., Grade, M., Thieffry, D., Grundhoff, A., Wegwitz, F., Johnsen, S.A., 2016. Histone Chaperone SSRP1 is Essential for Wnt Signaling Pathway Activity During Osteoblast Differentiation. Stem cells (Dayton, Ohio) 34 (5), 1369-1376. 10.1002/stem.2287.

Hoyert, D.L., Xu, J., 2012. Deaths: preliminary data for 2011. National vital statistics reports : from the Centers for Disease Control and Prevention, National Center for Health Statistics, National Vital Statistics System 61 (6), 1-51.

Hu, J., Liu, Y.-L., Piao, S.-I., Yang, D.-d., Yang, Y.-M., Cai, L., 2012. Expression patterns of USP22 and potential targets BMI-1, PTEN, p-AKT in non-small-cell lung cancer. Lung cancer (Amsterdam, Netherlands) 77 (3), 593-599. 10.1016/j.lungcan.2012.05.112.

Human Microbiome Project Consortium, 2012. Structure, function and diversity of the healthy human microbiome. Nature 486 (7402), 207-214. 10.1038/nature11234.

Hussain, S.P., Amstad, P., Raja, K., Ambs, S., Nagashima, M., Bennett, W.P., Shields, P.G., Ham, A.J., Swenberg, J.A., Marrogi, A.J., Harris, C.C., 2000. Increased p53 mutation load in noncancerous colon tissue from ulcerative colitis: a cancer-prone chronic inflammatory disease. Cancer research 60 (13), 3333-3337.

Ibrahim, A., Barnes, D.R., Dunlop, J., Barrowdale, D., Antoniou, A.C., Berg, J.N., 2014. Attenuated familial adenomatous polyposis manifests as autosomal dominant late-onset colorectal cancer. European journal of human genetics : EJHG 22 (11), 1330-1333. 10.1038/ejhg.2014.20.

Ichikawa-Tomikawa, N., Sugimoto, K., Satohisa, S., Nishiura, K., Chiba, H., 2011. Possible involvement of tight junctions, extracellular matrix and nuclear receptors in epithelial differentiation. Journal of biomedicine \& biotechnology 2011, 253048. $10.1155 / 2011 / 253048$. 
Issa, J.-P., 2008. Colon cancer: it's CIN or CIMP. Clinical cancer research : an official journal of the American Association for Cancer Research 14 (19), 5939-5940. 10.1158/10780432.CCR-08-1596.

Itzen, F., Greifenberg, A.K., Bosken, C.A., Geyer, M., 2014. Brd4 activates P-TEFb for RNA polymerase II CTD phosphorylation. Nucleic acids research 42 (12), 7577-7590. 10.1093/nar/gku449.

Janne, P.A., Mayer, R.J., 2000. Chemoprevention of colorectal cancer. The New England journal of medicine 342 (26), 1960-1968. 10.1056/NEJM200006293422606.

Jensen, A.B., Larsen, M., Gislum, M., Skriver, M.V., Jepsen, P., Norgaard, B., Sorensen, H.T., 2006. Survival after colorectal cancer in patients with ulcerative colitis: a nationwide population-based Danish study. The American journal of gastroenterology 101 (6), 12831287. 10.1111/j.1572-0241.2006.00520.x.

Johansson, M.E.V., Ambort, D., Pelaseyed, T., Schutte, A., Gustafsson, J.K., Ermund, A., Subramani, D.B., Holmen-Larsson, J.M., Thomsson, K.A., Bergstrom, J.H., van der Post, S., Rodriguez-Pineiro, A.M., Sjovall, H., Backstrom, M., Hansson, G.C., 2011. Composition and functional role of the mucus layers in the intestine. Cellular and molecular life sciences : CMLS 68 (22), 3635-3641. 10.1007/s00018-011-0822-3.

Jong, A.E. de, Hendriks, Y.M.C., Kleibeuker, J.H., Boer, S.Y. de, Cats, A., Griffioen, G., Nagengast, F.M., Nelis, F.G., Rookus, M.A., Vasen, H.F.A., 2006. Decrease in mortality in Lynch syndrome families because of surveillance. Gastroenterology 130 (3), 665-671. 10.1053/j.gastro.2005.11.032.

Jung, M., Gelato, K.A., Fernandez-Montalvan, A., Siegel, S., Haendler, B., 2015. Targeting BET bromodomains for cancer treatment. Epigenomics 7 (3), 487-501. 10.2217/epi.14.91.

Juo, Y.Y., Johnston, F.M., Zhang, D.Y., Juo, H.H., Wang, H., Pappou, E.P., Yu, T., Easwaran, H., Baylin, S., van Engeland, M., Ahuja, N., 2014. Prognostic value of CpG island methylator phenotype among colorectal cancer patients: a systematic review and meta-analysis. Annals of oncology : official journal of the European Society for Medical Oncology 25 (12), 2314-2327. 10.1093/annonc/mdu149.

Kabbarah, O., Mallon, M.A., Pfeifer, J.D., Edelmann, W., Kucherlapati, R., Goodfellow, P.J., 2003. A panel of repeat markers for detection of microsatellite instability in murine tumors. Molecular carcinogenesis 38 (4), 155-159. 10.1002/mc.10157.

Kaelin, W.G., JR, 2005. The concept of synthetic lethality in the context of anticancer therapy. Nature reviews. Cancer 5 (9), 689-698. 10.1038/nrc1691.

Kaler, P., Godasi, B.N., Augenlicht, L., Klampfer, L., 2009. The NF-kappaB/AKT-dependent Induction of Wnt Signaling in Colon Cancer Cells by Macrophages and IL-1beta. Cancer microenvironment : official journal of the International Cancer Microenvironment Society 2 (1), 69-80. 10.1007/s12307-009-0030-y. 
Kang, H., O'Connell, J.B., Maggard, M.A., Sack, J., Ko, C.Y., 2005. A 10-year outcomes evaluation of mucinous and signet-ring cell carcinoma of the colon and rectum. Diseases of the colon and rectum 48 (6), 1161-1168. 10.1007/s10350-004-0932-1.

Karpiuk, O., Najafova, Z., Kramer, F., Hennion, M., Galonska, C., Konig, A., Snaidero, N., Vogel, T., Shchebet, A., Begus-Nahrmann, Y., Kassem, M., Simons, M., Shcherbata, H., Beissbarth, T., Johnsen, S.A., 2012. The histone H2B monoubiquitination regulatory pathway is required for differentiation of multipotent stem cells. Molecular cell 46 (5), 705713. 10.1016/j.molcel.2012.05.022.

Kearns, K., Dee, A., Fitzgerald, A.P., Doherty, E., Perry, I.J., 2014. Chronic disease burden associated with overweight and obesity in Ireland: the effects of a small BMI reduction at population level. BMC public health 14, 143. 10.1186/1471-2458-14-143.

Kim, D.H., Pickhardt, P.J., Taylor, A.J., Leung, W.K., Winter, T.C., Hinshaw, J.L., Gopal, D.V., Reichelderfer, M., Hsu, R.H., Pfau, P.R., 2007. CT colonography versus colonoscopy for the detection of advanced neoplasia. The New England journal of medicine 357 (14), 1403-1412. 10.1056/NEJMoa070543.

Kim, Y.J., Hong, K.S., Chung, J.W., Kim, J.H., Hahm, K.B., 2010. Prevention of colitisassociated carcinogenesis with infliximab. Cancer prevention research (Philadelphia, Pa.) 3 (10), 1314-1333. 10.1158/1940-6207.CAPR-09-0272.

Klampfer, L., 2011. Cytokines, inflammation and colon cancer. Current cancer drug targets 11 (4), 451-464.

Kolligs, F.T., 2016. Diagnostics and Epidemiology of Colorectal Cancer. Visceral medicine 32 (3), 158-164. 10.1159/000446488.

Komrakova, M., Stuermer, E.K., Werner, C., Wicke, M., Kolios, L., Sehmisch, S., Tezval, M., Daub, F., Martens, T., Witzenhausen, P., Dullin, C., Stuermer, K.M., 2010. Effect of human parathyroid hormone hPTH (1-34) applied at different regimes on fracture healing and muscle in ovariectomized and healthy rats. Bone 47 (3), 480-492. 10.1016/j.bone.2010.05.013.

Kosinsky, R.L., 2013. USP22 and its role in organ maintenance and cellular function. M.Sc. thesis.

Kosinsky, R.L., Wegwitz, F., Hellbach, N., Dobbelstein, M., Mansouri, A., Vogel, T., BegusNahrmann, Y., Johnsen, S.A., 2015. Usp22 deficiency impairs intestinal epithelial lineage specification in vivo. Oncotarget 6 (35), 37906-37918. 10.18632/oncotarget.5412.

Landre, T., Uzzan, B., Nicolas, P., Aparicio, T., Zelek, L., Mary, F., Taleb, C., Des Guetz, G., 2015. Doublet chemotherapy vs. single-agent therapy with 5FU in elderly patients with metastatic colorectal cancer. a meta-analysis. International journal of colorectal disease 30 (10), 1305-1310. 10.1007/s00384-015-2296-5.

Landy, J., Ronde, E., English, N., Clark, S.K., Hart, A.L., Knight, S.C., Ciclitira, P.J., Al-Hassi, H.O., 2016. Tight junctions in inflammatory bowel diseases and inflammatory bowel disease associated colorectal cancer. World journal of gastroenterology 22 (11), 31173126. 10.3748/wjg.v22.i11.3117. 
Launois, R., Le Moine, J.-G., Uzzan, B., Fiestas Navarrete, L.I., Benamouzig, R., 2014. Systematic review and bivariate/HSROC random-effect meta-analysis of immunochemical and guaiac-based fecal occult blood tests for colorectal cancer screening. European journal of gastroenterology \& hepatology 26 (9), 978-989. 10.1097/MEG.0000000000000160.

Lee, D.H., Qi, J., Bradner, J.E., Said, J.W., Doan, N.B., Forscher, C., Yang, H., Koeffler, H.P., 2015. Synergistic effect of JQ1 and rapamycin for treatment of human osteosarcoma. International journal of cancer 136 (9), 2055-2064. 10.1002/ijc.29269.

Lee, G., Goretsky, T., Managlia, E., Dirisina, R., Singh, A.P., Brown, J.B., May, R., Yang, G.Y., Ragheb, J.W., Evers, B.M., Weber, C.R., Turner, J.R., He, X.C., Katzman, R.B., Li, L., Barrett, T.A., 2010. Phosphoinositide 3-kinase signaling mediates beta-catenin activation in intestinal epithelial stem and progenitor cells in colitis. Gastroenterology 139 (3), 86981, 881.e1-9. 10.1053/j.gastro.2010.05.037.

Lee, H.-J., Kim, M.-S., Shin, J.-M., Park, T.-J., Chung, H.-M., Baek, K.-H., 2006. The expression patterns of deubiquitinating enzymes, USP22 and Usp22. Gene expression patterns : GEP 6 (3), 277-284. 10.1016/j.modgep.2005.07.007.

Lee, J.M., Lee, K.-M., 2016. Endoscopic Diagnosis and Differentiation of Inflammatory Bowel Disease. Clinical endoscopy 49 (4), 370-375. 10.5946/ce.2016.090.

Leopoldo, S., Lorena, B., Cinzia, A., Di Gabriella, C., Angela Luciana, B., Renato, C., Antonio, M., Carlo, S., Cristina, P., Stefano, C., Maurizio, T., Luigi, R., Cesare, B., 2008. Two subtypes of mucinous adenocarcinoma of the colorectum: clinicopathological and genetic features. Annals of surgical oncology 15 (5), 1429-1439. 10.1245/s10434-0079757-1.

Li, L., Osdal, T., Ho, Y., Chun, S., McDonald, T., Agarwal, P., Lin, A., Chu, S., Qi, J., Li, L., Hsieh, Y.-T., Dos Santos, C., Yuan, H., Ha, T.-Q., Popa, M., Hovland, R., Bruserud, O., Gjertsen, B.T., Kuo, Y.-H., Chen, W., Lain, S., McCormack, E., Bhatia, R., 2014a. SIRT1 activation by a c-MYC oncogenic network promotes the maintenance and drug resistance of human FLT3-ITD acute myeloid leukemia stem cells. Cell stem cell 15 (4), 431-446. 10.1016/j.stem.2014.08.001.

Li, L., Wen, S., Wang, B., Gao, W., Zhang, W., Meng, X., Yang, L., Kong, L., 2014b. Expression of cancer stem cell marker USP22 in laryngeal squamous cell carcinoma. Zhonghua er bi yan hou tou jing wai ke za zhi = Chinese journal of otorhinolaryngology head and neck surgery 49 (6), 479-482.

Li, M., Brooks, C.L., Kon, N., Gu, W., 2004. A dynamic role of HAUSP in the p53-Mdm2 pathway. Molecular cell 13 (6), 879-886.

Liebmann, C., 2001. Regulation of MAP kinase activity by peptide receptor signalling pathway: paradigms of multiplicity. Cellular signalling 13 (11), 777-785.

Lilja, A., Weeden, C.E., McArthur, K., Nguyen, T., Donald, A., Wong, Z.X., Dousha, L., Bozinovski, S., Vlahos, R., Burns, C.J., Asselin-Labat, M.-L., Anderson, G.P., 2015. HSP90 inhibition suppresses lipopolysaccharide-induced lung inflammation in vivo. PloS one 10 (1), e0114975. 10.1371/journal.pone.0114975. 
Lin, Z., Tan, C., Qiu, Q., Kong, S., Yang, H., Zhao, F., Liu, Z., Li, J., Kong, Q., Gao, B., Barrett, T., Yang, G.-Y., Zhang, J., Fang, D., 2015. Ubiquitin-specific protease 22 is a deubiquitinase of CCNB1. Cell discovery 1. 10.1038/celldisc.2015.28.

Lin, Z., Yang, H., Kong, Q., Li, J., Lee, S.-M., Gao, B., Dong, H., Wei, J., Song, J., Zhang, D.D., Fang, D., 2012. USP22 antagonizes p53 transcriptional activation by deubiquitinating Sirt1 to suppress cell apoptosis and is required for mouse embryonic development. Molecular cell 46 (4), 484-494. 10.1016/j.molcel.2012.03.024.

Lister, R., Pelizzola, M., Dowen, R.H., Hawkins, R.D., Hon, G., Tonti-Filippini, J., Nery, J.R., Lee, L., Ye, Z., Ngo, Q.-M., Edsall, L., Antosiewicz-Bourget, J., Stewart, R., Ruotti, V., Millar, A.H., Thomson, J.A., Ren, B., Ecker, J.R., 2009. Human DNA methylomes at base resolution show widespread epigenomic differences. Nature 462 (7271), 315-322. 10.1038/nature08514.

Liu, J., Shaik, S., Dai, X., Wu, Q., Zhou, X., Wang, Z., Wei, W., 2015a. Targeting the ubiquitin pathway for cancer treatment. Biochimica et biophysica acta 1855 (1), 50-60. 10.1016/j.bbcan.2014.11.005.

Liu, J.Z., van Sommeren, S., Huang, H., Ng, S.C., Alberts, R., Takahashi, A., Ripke, S., Lee, J.C., Jostins, L., Shah, T., Abedian, S., Cheon, J.H., Cho, J., Daryani, N.E., Franke, L., Fuyuno, Y., Hart, A., Juyal, R.C., Juyal, G., Kim, W.H., Morris, A.P., Poustchi, H., Newman, W.G., Midha, V., Orchard, T.R., Vahedi, H., Sood, A., Sung, J.J.Y., Malekzadeh, R., Westra, H.-J., Yamazaki, K., Yang, S.-K., Barrett, J.C., Franke, A., Alizadeh, B.Z., Parkes, M., B K, T., Daly, M.J., Kubo, M., Anderson, C.A., Weersma, R.K., 2015b. Association analyses identify 38 susceptibility loci for inflammatory bowel disease and highlight shared genetic risk across populations. Nature genetics 47 (9), 979-986. 10.1038/ng.3359.

Liu, Y., Yang, Y., Xu, H., Dong, X., 2010. Implication of USP22 in the regulation of BMI-1, cMyc, p16INK4a, p14ARF, and cyclin D2 expression in primary colorectal carcinomas.

Diagnostic molecular pathology : the American journal of surgical pathology, part B 19 (4), 194-200. 10.1097/PDM.0b013e3181e202f2.

Liu, Y.-L., Jiang, S.-X., Yang, Y.-M., Xu, H., Liu, J.-L., Wang, X.-S., 2012. USP22 acts as an oncogene by the activation of BMI-1-mediated INK4a/ARF pathway and Akt pathway. Cell biochemistry and biophysics 62 (1), 229-235. 10.1007/s12013-011-9287-0.

Liu, Y.-L., Yang, Y.-M., Xu, H., Dong, X.-S., 2011. Aberrant expression of USP22 is associated with liver metastasis and poor prognosis of colorectal cancer. Journal of surgical oncology 103 (3), 283-289. 10.1002/jso.21802.

Lucke, K., Miehlke, S., Jacobs, E., Schuppler, M., 2006. Prevalence of Bacteroides and Prevotella spp. in ulcerative colitis. Journal of medical microbiology 55 (Pt 5), 617-624. 10.1099/jmm.0.46198-0.

Luger, K., Mader, A.W., Richmond, R.K., Sargent, D.F., Richmond, T.J., 1997. Crystal structure of the nucleosome core particle at 2.8 A resolution. Nature 389 (6648), 251-260. $10.1038 / 38444$. 
Lutgens, Maurice W M D, van Oijen, Martijn G H, van der Heijden, Geert J M G, Vleggaar, F.P., Siersema, P.D., Oldenburg, B., 2013. Declining risk of colorectal cancer in inflammatory bowel disease: an updated meta-analysis of population-based cohort studies. Inflammatory bowel diseases 19 (4), 789-799. 10.1097/MIB.0b013e31828029c0.

Lv, L., Xiao, X.-Y., Gu, Z.-H., Zeng, F.-Q., Huang, L.-Q., Jiang, G.-S., 2011. Silencing USP22 by asymmetric structure of interfering RNA inhibits proliferation and induces cell cycle arrest in bladder cancer cells. Molecular and cellular biochemistry 346 (1-2), 11-21. 10.1007/s11010-010-0585-4.

Lynch, H.T., 1967. Hereditary Factors in Carcinoma. Springer Berlin Heidelberg, Berlin, Heidelberg, 1 online resource (volumes).

Lynch, H.T., Lynch, P.M., Lanspa, S.J., Snyder, C.L., Lynch, J.F., Boland, C.R., 2009. Review of the Lynch syndrome: history, molecular genetics, screening, differential diagnosis, and medicolegal ramifications. Clinical genetics 76 (1), 1-18. 10.1111/j.13990004.2009.01230.x.

Mair, B., Kubicek, S., Nijman, S.M.B., 2014. Exploiting epigenetic vulnerabilities for cancer therapeutics. Trends in pharmacological sciences 35 (3), 136-145. 10.1016/j.tips.2014.01.001.

Mandel, J.S., Bond, J.H., Church, T.R., Snover, D.C., Bradley, G.M., Schuman, L.M., Ederer, F., 1993. Reducing mortality from colorectal cancer by screening for fecal occult blood. Minnesota Colon Cancer Control Study. The New England journal of medicine 328 (19), 1365-1371. 10.1056/NEJM199305133281901.

Marrie, R.A., Walker, J.R., Graff, L.A., Lix, L.M., Bolton, J.M., Nugent, Z., Targownik, L.E., Bernstein, C.N., 2016. Performance of administrative case definitions for depression and anxiety in inflammatory bowel disease. Journal of psychosomatic research $89,107-113$. 10.1016/j.jpsychores.2016.08.014.

Mayo, M.W., Wang, C.Y., Cogswell, P.C., Rogers-Graham, K.S., Lowe, S.W., Der, C.J., Baldwin, A.S., JR, 1997. Requirement of NF-kappaB activation to suppress p53independent apoptosis induced by oncogenic Ras. Science (New York, N.Y.) 278 (5344), $1812-1815$.

Mazzola, P., Radhi, S., Mirandola, L., Annoni, G., Jenkins, M., Cobos, E., Chiriva-Internati, M., 2012. Aging, cancer, and cancer vaccines. Immunity \& ageing : I \& A 9 (1), 4. 10.1186/1742-4933-9-4.

McClurg, U.L., Robson, C.N., 2015. Deubiquitinating enzymes as oncotargets. Oncotarget 6 (12), 9657-9668. 10.18632/oncotarget.3922.

McDowell, C.L., Bryan Sutton, R., Obermann, W.M.J., 2009. Expression of Hsp90 chaperone corrected proteins in human tumor tissue. International journal of biological macromolecules 45 (3), 310-314. 10.1016/j.ijbiomac.2009.06.012.

Meira, L.B., Bugni, J.M., Green, S.L., Lee, C.-W., Pang, B., Borenshtein, D., Rickman, B.H., Rogers, A.B., Moroski-Erkul, C.A., McFaline, J.L., Schauer, D.B., Dedon, P.C., Fox, J.G., Samson, L.D., 2008. DNA damage induced by chronic inflammation contributes to colon 
carcinogenesis in mice. The Journal of clinical investigation 118 (7), 2516-2525. $10.1172 / \mathrm{JCl} 35073$.

Melling, N., Grimm, N., Simon, R., Stahl, P., Bokemeyer, C., Terracciano, L., Sauter, G., Izbicki, J.R., Marx, A.H., 2016. Loss of H2Bub1 Expression is Linked to Poor Prognosis in Nodal Negative Colorectal Cancers. Pathology oncology research : POR 22 (1), 95-102. 10.1007/s12253-015-9977-9.

Melo-Cardenas, J., Zhang, Y., Zhang, D.D., Fang, D., 2016. Ubiquitin-specific peptidase 22 functions and its involvement in disease. Oncotarget 7 (28), 44848-44856. 10.18632/oncotarget.8602.

Merga, Y.J., O'Hara, A., Burkitt, M.D., Duckworth, C.A., Probert, C.S., Campbell, B.J., Pritchard, D.M., 2016. Importance of the alternative NF-kappaB activation pathway in inflammation-associated gastrointestinal carcinogenesis. American journal of physiology. Gastrointestinal and liver physiology 310 (11), G1081-90. 10.1152/ajpgi.00026.2016.

Mesange, P., Poindessous, V., Sabbah, M., Escargueil, A.E., Gramont, A. de, Larsen, A.K., 2014. Intrinsic bevacizumab resistance is associated with prolonged activation of autocrine VEGF signaling and hypoxia tolerance in colorectal cancer cells and can be overcome by nintedanib, a small molecule angiokinase inhibitor. Oncotarget 5 (13), 47094721. 10.18632/oncotarget.1671.

Mesquita, M.B. de, Civitelli, F., Levine, A., 2008. Epidemiology, genes and inflammatory bowel diseases in childhood. Digestive and liver disease : official journal of the Italian Society of Gastroenterology and the Italian Association for the Study of the Liver 40 (1), 3-11. 10.1016/j.dld.2007.07.165.

Miclea, R.L., Karperien, M., Langers, A.M., Robanus-Maandag, E.C., van Lierop, A., van der Hiel, B., Stokkel, M.P., Ballieux, B.E., Oostdijk, W., Wit, J.M., Vasen, H.F., Hamdy, N.A., 2010. APC mutations are associated with increased bone mineral density in patients with familial adenomatous polyposis. Journal of bone and mineral research : the official journal of the American Society for Bone and Mineral Research 25 (12), 2624-2632. 10.1002/jbmr.153.

Mimnaugh, E.G., Chavany, C., Neckers, L., 1996. Polyubiquitination and proteasomal degradation of the $185 \mathrm{c}$-erbB-2 receptor protein-tyrosine kinase induced by geldanamycin. The Journal of biological chemistry 271 (37), 22796-22801.

Mishra, V.K., Johnsen, S.A., 2014. Targeted therapy of epigenomic regulatory mechanisms controlling the epithelial to mesenchymal transition during tumor progression. Cell and tissue research 356 (3), 617-630. 10.1007/s00441-014-1912-y.

Mohiuddin, M., Winter, K., Mitchell, E., Hanna, N., Yuen, A., Nichols, C., Shane, R., Hayostek, C., Willett, C., 2006. Randomized phase II study of neoadjuvant combinedmodality chemoradiation for distal rectal cancer: Radiation Therapy Oncology Group Trial 0012. Journal of clinical oncology : official journal of the American Society of Clinical Oncology 24 (4), 650-655. 10.1200/JCO.2005.03.6095.

Molodecky, N.A., Soon, I.S., Rabi, D.M., Ghali, W.A., Ferris, M., Chernoff, G., Benchimol, E.I., Panaccione, R., Ghosh, S., Barkema, H.W., Kaplan, G.G., 2012. Increasing 
incidence and prevalence of the inflammatory bowel diseases with time, based on systematic review. Gastroenterology 142 (1), 46-54.e42; quiz e30. 10.1053/j.gastro.2011.10.001.

Mora, J.R., Andrian, U.H. von, 2008. Differentiation and homing of IgA-secreting cells. Mucosal immunology 1 (2), 96-109. 10.1038/mi.2007.14.

Morales, F., Giordano, A., 2016. Overview of CDK9 as a target in cancer research. Cell cycle (Georgetown, Tex.) 15 (4), 519-527. 10.1080/15384101.2016.1138186.

Munteanu, I., Mastalier, B., 2014. Genetics of colorectal cancer. Journal of medicine and life 7 (4), 507-511.

Murata, S., Zhang, C., Finch, N., Zhang, K., Campo, L., Breuer, E.-K., 2016. Predictors and Modulators of Synthetic Lethality: An Update on PARP Inhibitors and Personalized Medicine. BioMed research international 2016, 2346585. 10.1155/2016/2346585.

Myrelid, P., Salim, S.Y., Darby, T., Almer, S., Melgar, S., Andersson, P., Soderholm, J.D., 2015. Effects of anti-inflammatory therapy on bursting pressure of colonic anastomosis in murine dextran sulfate sodium induced colitis. Scandinavian journal of gastroenterology 50 (8), 991-1001. 10.3109/00365521.2014.964760.

Nechaev, S., Adelman, K., 2011. Pol II waiting in the starting gates: Regulating the transition from transcription initiation into productive elongation. Biochimica et biophysica acta 1809 (1), 34-45. 10.1016/j.bbagrm.2010.11.001.

Neurath, M.F., 2014. Cytokines in inflammatory bowel disease. Nature reviews. Immunology 14 (5), 329-342. 10.1038/nri3661.

Ng, M., Fleming, T., Robinson, M., Thomson, B., Graetz, N., Margono, C., Mullany, E.C., Biryukov, S., Abbafati, C., Abera, S.F., Abraham, J.P., Abu-Rmeileh, N.M.E., Achoki, T., AlBuhairan, F.S., Alemu, Z.A., Alfonso, R., Ali, M.K., Ali, R., Guzman, N.A., Ammar, W., Anwari, P., Banerjee, A., Barquera, S., Basu, S., Bennett, D.A., Bhutta, Z., Blore, J., Cabral, N., Nonato, I.C., Chang, J.-C., Chowdhury, R., Courville, K.J., Criqui, M.H., Cundiff, D.K., Dabhadkar, K.C., Dandona, L., Davis, A., Dayama, A., Dharmaratne, S.D., Ding, E.L., Durrani, A.M., Esteghamati, A., Farzadfar, F., Fay, D.F.J., Feigin, V.L., Flaxman, A., Forouzanfar, M.H., Goto, A., Green, M.A., Gupta, R., Hafezi-Nejad, N., Hankey, G.J., Harewood, H.C., Havmoeller, R., Hay, S., Hernandez, L., Husseini, A., Idrisov, B.T., Ikeda, N., Islami, F., Jahangir, E., Jassal, S.K., Jee, S.H., Jeffreys, M., Jonas, J.B., Kabagambe, E.K., Khalifa, Shams Eldin Ali Hassan, Kengne, A.P., Khader, Y.S., Khang, Y.-H., Kim, D., Kimokoti, R.W., Kinge, J.M., Kokubo, Y., Kosen, S., Kwan, G., Lai, T., Leinsalu, M., Li, Y., Liang, X., Liu, S., Logroscino, G., Lotufo, P.A., Lu, Y., Ma, J., Mainoo, N.K., Mensah, G.A., Merriman, T.R., Mokdad, A.H., Moschandreas, J., Naghavi, M., Naheed, A., Nand, D., Narayan, K.M.V., Nelson, E.L., Neuhouser, M.L., Nisar, M.I., Ohkubo, T., Oti, S.O., Pedroza, A., Prabhakaran, D., Roy, N., Sampson, U., Seo, H., Sepanlou, S.G., Shibuya, K., Shiri, R., Shiue, I., Singh, G.M., Singh, J.A., Skirbekk, V., Stapelberg, N.J.C., Sturua, L., Sykes, B.L., Tobias, M., Tran, B.X., Trasande, L., Toyoshima, H., van de Vijver, S., Vasankari, T.J., Veerman, J.L., Velasquez-Melendez, G., Vlassov, V.V., Vollset, S.E., Vos, T., Wang, C., Wang, X., Weiderpass, E., Werdecker, A., Wright, J.L., Yang, Y.C., Yatsuya, H., Yoon, J., Yoon, S.J., Zhao, Y., Zhou, M., Zhu, S., Lopez, A.D., Murray, C.J.L., Gakidou, E., 2014. Global, 
regional, and national prevalence of overweight and obesity in children and adults during 1980-2013: a systematic analysis for the Global Burden of Disease Study 2013. Lancet (London, England) 384 (9945), 766-781. 10.1016/S0140-6736(14)60460-8.

Nijman, S.M.B., Luna-Vargas, M.P.A., Velds, A., Brummelkamp, T.R., Dirac, A.M.G., Sixma, T.K., Bernards, R., 2005. A genomic and functional inventory of deubiquitinating enzymes. Cell 123 (5), 773-786. 10.1016/j.cell.2005.11.007.

Ning, Z., Wang, A., Liang, J., Xie, Y., Liu, J., Feng, L., Yan, Q., Wang, Z., 2014a. USP22 promotes the G1/S phase transition by upregulating FoxM1 expression via beta-catenin nuclear localization and is associated with poor prognosis in stage II pancreatic ductal adenocarcinoma. International journal of oncology 45 (4), 1594-1608. 10.3892/ijo.2014.2531.

Ning, Z., Wang, A., Liang, J., Xie, Y., Liu, J., Yan, Q., Wang, Z., 2014b. USP22 promotes epithelial-mesenchymal transition via the FAK pathway in pancreatic cancer cells. Oncology reports 32 (4), 1451-1458. 10.3892/or.2014.3354.

Nordgren, S., McPheeters, G., Svaninger, G., Oresland, T., Hulten, L., 1997. Small bowel length in inflammatory bowel disease. International journal of colorectal disease 12 (4), 230-234.

Novelli, M., 2015. The pathology of hereditary polyposis syndromes. Histopathology 66 (1), 78-87. 10.1111/his.12590.

O'Connell, J.B., Maggard, M.A., Ko, C.Y., 2004. Colon cancer survival rates with the new American Joint Committee on Cancer sixth edition staging. Journal of the National Cancer Institute 96 (19), 1420-1425. 10.1093/jnci/djh275.

Ohhara, Y., Fukuda, N., Takeuchi, S., Honma, R., Shimizu, Y., Kinoshita, I., Dosaka-Akita, $\mathrm{H}$., 2016. Role of targeted therapy in metastatic colorectal cancer. World journal of gastrointestinal oncology 8 (9), 642-655. 10.4251/wjgo.v8.i9.642.

Okayasu, I., Hatakeyama, S., Yamada, M., Ohkusa, T., Inagaki, Y., Nakaya, R., 1990. A novel method in the induction of reliable experimental acute and chronic ulcerative colitis in mice. Gastroenterology 98 (3), 694-702.

O'Keeffe, B., Fong, Y., Chen, D., Zhou, S., Zhou, Q., 2000. Requirement for a kinase-specific chaperone pathway in the production of a Cdk9/cyclin T1 heterodimer responsible for $\mathrm{P}$ TEFb-mediated tat stimulation of HIV-1 transcription. The Journal of biological chemistry 275 (1), 279-287.

Okugawa, Y., Grady, W.M., Goel, A., 2015. Epigenetic Alterations in Colorectal Cancer: Emerging Biomarkers. Gastroenterology 149 (5), 1204-1225.e12. 10.1053/j.gastro.2015.07.011.

Oldenburg, B., Koningsberger, J.C., Van Berge Henegouwen, G P, van Asbeck, B.S., Marx, J.J., 2001. Iron and inflammatory bowel disease. Alimentary pharmacology \& therapeutics 15 (4), 429-438. 
Orphanides, G., LeRoy, G., Chang, C.H., Luse, D.S., Reinberg, D., 1998. FACT, a factor that facilitates transcript elongation through nucleosomes. Cell 92 (1), 105-116.

Ortega-Gomez, A., Perretti, M., Soehnlein, O., 2013. Resolution of inflammation: an integrated view. EMBO molecular medicine 5 (5), 661-674. 10.1002/emmm.201202382.

Paepe, B. de, Creus, K.K., Martin, J.-J., Weis, J., Bleecker, J.L. de, 2009. A dual role for HSP90 and HSP70 in the inflammatory myopathies: from muscle fiber protection to active invasion by macrophages. Annals of the New York Academy of Sciences 1173, 463-469. 10.1111/j.1749-6632.2009.04812.x.

Pancione, M., Remo, A., Colantuoni, V., 2012. Genetic and epigenetic events generate multiple pathways in colorectal cancer progression. Pathology research international 2012, 509348. 10.1155/2012/509348.

Panda, S., El khader, I., Casellas, F., Lopez Vivancos, J., Garcia Cors, M., Santiago, A., Cuenca, S., Guarner, F., Manichanh, C., 2014. Short-term effect of antibiotics on human gut microbiota. PloS one 9 (4), e95476. 10.1371/journal.pone.0095476.

Patel, S.G., Ahnen, D.J., 2012. Familial colon cancer syndromes: an update of a rapidly evolving field. Current gastroenterology reports 14 (5), 428-438. 10.1007/s11894-0120280-6.

Pavri, R., Zhu, B., Li, G., Trojer, P., Mandal, S., Shilatifard, A., Reinberg, D., 2006. Histone $\mathrm{H} 2 \mathrm{~B}$ monoubiquitination functions cooperatively with FACT to regulate elongation by RNA polymerase II. Cell 125 (4), 703-717. 10.1016/j.cell.2006.04.029.

Perse, M., Cerar, A., 2012. Dextran sodium sulphate colitis mouse model: traps and tricks. Journal of biomedicine \& biotechnology 2012, 718617. 10.1155/2012/718617.

Petersson, J., Schreiber, O., Hansson, G.C., Gendler, S.J., Velcich, A., Lundberg, J.O., Roos, S., Holm, L., Phillipson, M., 2011. Importance and regulation of the colonic mucus barrier in a mouse model of colitis. American journal of physiology. Gastrointestinal and liver physiology 300 (2), G327-33. 10.1152/ajpgi.00422.2010.

Peuker, K., Muff, S., Wang, J., Kunzel, S., Bosse, E., Zeissig, Y., Luzzi, G., Basic, M., Strigli, A., Ulbricht, A., Kaser, A., Arlt, A., Chavakis, T., van den Brink, Gijs R, Schafmayer, C., Egberts, J.-H., Becker, T., Bianchi, M.E., Bleich, A., Rocken, C., Hampe, J., Schreiber, S., Baines, J.F., Blumberg, R.S., Zeissig, S., 2016. Epithelial calcineurin controls microbiotadependent intestinal tumor development. Nature medicine 22 (5), 506-515. $10.1038 / \mathrm{nm} .4072$.

Pickart, C.M., 2001. Mechanisms underlying ubiquitination. Annual review of biochemistry 70, 503-533. 10.1146/annurev.biochem.70.1.503.

Prenzel, T., Begus-Nahrmann, Y., Kramer, F., Hennion, M., Hsu, C., Gorsler, T., Hintermair, C., Eick, D., Kremmer, E., Simons, M., Beissbarth, T., Johnsen, S.A., 2011. Estrogendependent gene transcription in human breast cancer cells relies upon proteasomedependent monoubiquitination of histone H2B. Cancer research 71 (17), 5739-5753. 10.1158/0008-5472.CAN-11-1896. 
Proia, D.A., Bates, R.C., 2014. Ganetespib and HSP90: translating preclinical hypotheses into clinical promise. Cancer research 74 (5), 1294-1300. 10.1158/0008-5472.CAN-133263.

Qadri, S.M., Mahmud, H., Lang, E., Gu, S., Bobbala, D., Zelenak, C., Jilani, K., Siegfried, A., Foller, M., Lang, F., 2012. Enhanced suicidal erythrocyte death in mice carrying a loss-offunction mutation of the adenomatous polyposis coli gene. Journal of cellular and molecular medicine 16 (5), 1085-1093. 10.1111/j.1582-4934.2011.01387.x.

Qi, D., Larsson, J., Mannervik, M., 2004. Drosophila Ada2b is required for viability and normal histone H3 acetylation. Molecular and cellular biology 24 (18), 8080-8089. 10.1128/MCB.24.18.8080-8089.2004.

Qin, J., Li, R., Raes, J., Arumugam, M., Burgdorf, K.S., Manichanh, C., Nielsen, T., Pons, N., Levenez, F., Yamada, T., Mende, D.R., Li, J., Xu, J., Li, S., Li, D., Cao, J., Wang, B., Liang, H., Zheng, H., Xie, Y., Tap, J., Lepage, P., Bertalan, M., Batto, J.-M., Hansen, T., Le Paslier, D., Linneberg, A., Nielsen, H.B., Pelletier, E., Renault, P., Sicheritz-Ponten, T., Turner, K., Zhu, H., Yu, C., Li, S., Jian, M., Zhou, Y., Li, Y., Zhang, X., Li, S., Qin, N., Yang, H., Wang, J., Brunak, S., Dore, J., Guarner, F., Kristiansen, K., Pedersen, O., Parkhill, J., Weissenbach, J., Bork, P., Ehrlich, S.D., Wang, J., 2010. A human gut microbial gene catalogue established by metagenomic sequencing. Nature 464 (7285), 59-65. 10.1038/nature08821.

Rakoff-Nahoum, S., Bousvaros, A., 2010. Innate and adaptive immune connections in inflammatory bowel diseases. Current opinion in gastroenterology 26 (6), 572-577. 10.1097/MOG.0b013e32833f126d.

Ramalingam, S.S., Maitland, M.L., Frankel, P., Argiris, A.E., Koczywas, M., Gitlitz, B., Thomas, S., Espinoza-Delgado, I., Vokes, E.E., Gandara, D.R., Belani, C.P., 2010. Carboplatin and Paclitaxel in combination with either vorinostat or placebo for first-line therapy of advanced non-small-cell lung cancer. Journal of clinical oncology : official journal of the American Society of Clinical Oncology 28 (1), 56-62. 10.1200/JCO.2009.24.9094.

Rehman, A., Lepage, P., Nolte, A., Hellmig, S., Schreiber, S., Ott, S.J., 2010. Transcriptional activity of the dominant gut mucosal microbiota in chronic inflammatory bowel disease patients. Journal of medical microbiology 59 (Pt 9), 1114-1122. 10.1099/jmm.0.021170-0.

Renz, H., Mutius, E. von, Brandtzaeg, P., Cookson, W.O., Autenrieth, I.B., Haller, D., 2011. Gene-environment interactions in chronic inflammatory disease. Nature immunology 12 (4), 273-277. 10.1038/ni0411-273.

Reverdy, C., Conrath, S., Lopez, R., Planquette, C., Atmanene, C., Collura, V., Harpon, J., Battaglia, V., Vivat, V., Sippl, W., Colland, F., 2012. Discovery of specific inhibitors of human USP7/HAUSP deubiquitinating enzyme. Chemistry \& biology 19 (4), 467-477. 10.1016/j.chembiol.2012.02.007.

Reya, T., Clevers, H., 2005. Wnt signalling in stem cells and cancer. Nature 434 (7035), 843850. 10.1038/nature03319. 
Rhoades, R., Tanner, G.A., 2003. Medical physiology, 2nd ed. Lippincott Williams \& Wilkins, Philadelphia, $x, 781$.

Rhodes, D.R., Yu, J., Shanker, K., Deshpande, N., Varambally, R., Ghosh, D., Barrette, T., Pandey, A., Chinnaiyan, A.M., 2004. ONCOMINE: a cancer microarray database and integrated data-mining platform. Neoplasia (New York, N.Y.) 6 (1), 1-6.

Richardson, P.G., Hideshima, T., Anderson, K.C., 2003. Bortezomib (PS-341): a novel, firstin-class proteasome inhibitor for the treatment of multiple myeloma and other cancers. Cancer control : journal of the Moffitt Cancer Center 10 (5), 361-369.

Richardson, P.G., Hungria, V.T.M., Yoon, S.-S., Beksac, M., Dimopoulos, M.A., Elghandour, A., Jedrzejczak, W.W., Guenther, A., Nakorn, T.N., Siritanaratkul, N., Schlossman, R.L., Hou, J., Moreau, P., Lonial, S., Lee, J.H., Einsele, H., Sopala, M., Bengoudifa, B.-R., Corrado, C., Binlich, F., San-Miguel, J.F., 2016. Panobinostat plus bortezomib and dexamethasone in previously treated multiple myeloma: outcomes by prior treatment. Blood 127 (6), 713-721. 10.1182/blood-2015-09-665018.

Richmond, T.J., Davey, C.A., 2003. The structure of DNA in the nucleosome core. Nature 423 (6936), 145-150. 10.1038/nature01595.

Riihimaki, M., Hemminki, A., Sundquist, J., Hemminki, K., 2016. Patterns of metastasis in colon and rectal cancer. Scientific reports 6, 29765. 10.1038/srep29765.

Robanus-Maandag, E.C., Koelink, P.J., Breukel, C., Salvatori, D.C.F., Jagmohan-Changur, S.C., Bosch, C.A.J., Verspaget, H.W., Devilee, P., Fodde, R., Smits, R., 2010. A new conditional Apc-mutant mouse model for colorectal cancer. Carcinogenesis 31 (5), 946952. 10.1093/carcin/bgq046.

Robertis, M. de, Massi, E., Poeta, M.L., Carotti, S., Morini, S., Cecchetelli, L., Signori, E., Fazio, V.M., 2011. The AOM/DSS murine model for the study of colon carcinogenesis: From pathways to diagnosis and therapy studies. Journal of carcinogenesis 10, 9. 10.4103/1477-3163.78279.

Roda, G., Sartini, A., Zambon, E., Calafiore, A., Marocchi, M., Caponi, A., Belluzzi, A., Roda, E., 2010. Intestinal epithelial cells in inflammatory bowel diseases. World journal of gastroenterology 16 (34), 4264-4271.

Rogers, M.A.M., Aronoff, D.M., 2016. The influence of non-steroidal anti-inflammatory drugs on the gut microbiome. Clinical microbiology and infection : the official publication of the European Society of Clinical Microbiology and Infectious Diseases 22 (2), 178.e1-9. 10.1016/j.cmi.2015.10.003.

Rogler, G., 2014. Chronic ulcerative colitis and colorectal cancer. Cancer letters 345 (2), 235-241. 10.1016/j.canlet.2013.07.032.

Roslani, A.C., Abdullah, T., Arumugam, K., 2012. Screening for colorectal neoplasias with fecal occult blood tests: false-positive impact of non-dietary restriction. Asian Pacific journal of cancer prevention : APJCP 13 (1), 237-241. 
Round, J.L., Mazmanian, S.K., 2009. The gut microbiota shapes intestinal immune responses during health and disease. Nature reviews. Immunology 9 (5), 313-323. 10.1038/nri2515.

Rozen, P., Macrae, F., 2006. Familial adenomatous polyposis: The practical applications of clinical and molecular screening. Familial cancer 5 (3), 227-235. 10.1007/s10689-0055674-2.

Rozen, P., Samuel, Z., Rabau, M., Goldman, G., Shomrat, R., Legum, C., Orr-Urtreger, A., 2001. Familial adenomatous polyposis at the Tel Aviv Medical Center: demographic and clinical features. Familial cancer 1 (2), 75-82.

Ruemmele, F.M., 2010. Pediatric inflammatory bowel diseases: coming of age. Current opinion in gastroenterology 26 (4), 332-336. 10.1097/MOG.0b013e328339ec2d.

Ryan, K.M., Phillips, A.C., Vousden, K.H., 2001. Regulation and function of the p53 tumor suppressor protein. Current opinion in cell biology 13 (3), 332-337.

Sadanandam, A., Lyssiotis, C.A., Homicsko, K., Collisson, E.A., Gibb, W.J., Wullschleger, S., Ostos, L.C.G., Lannon, W.A., Grotzinger, C., Del Rio, M., Lhermitte, B., Olshen, A.B., Wiedenmann, B., Cantley, L.C., Gray, J.W., Hanahan, D., 2013. A colorectal cancer classification system that associates cellular phenotype and responses to therapy. Nature medicine 19 (5), 619-625. 10.1038/nm.3175.

Saidel-Odes, L., Odes, S., 2014. Hygiene hypothesis in inflammatory bowel disease. Annals of gastroenterology : quarterly publication of the Hellenic Society of Gastroenterology 27 (3), 189-190.

Samara, N.L., Datta, A.B., Berndsen, C.E., Zhang, X., Yao, T., Cohen, R.E., Wolberger, C., 2010. Structural insights into the assembly and function of the SAGA deubiquitinating module. Science (New York, N.Y.) 328 (5981), 1025-1029. 10.1126/science.1190049.

Sanderson, I.R., 2014. Growth problems in children with IBD. Nature reviews. Gastroenterology \& hepatology 11 (10), 601-610. 10.1038/nrgastro.2014.102.

San-Miguel, J.F., Hungria, V.T.M., Yoon, S.-S., Beksac, M., Dimopoulos, M.A., Elghandour, A., Jedrzejczak, W.W., Gunther, A., Nakorn, T.N., Siritanaratkul, N., Corradini, P., Chuncharunee, S., Lee, J.-J., Schlossman, R.L., Shelekhova, T., Yong, K., Tan, D., Numbenjapon, T., Cavenagh, J.D., Hou, J., LeBlanc, R., Nahi, H., Quu, L., Salwender, H., Pulini, S., Moreau, P., Warzocha, K., White, D., Blade, J., Chen, W., La Rubia, J. de, Gimsing, P., Lonial, S., Kaufman, J.L., Ocio, E.M., Veskovski, L., Sohn, S.K., Wang, M.C., Lee, J.H., Einsele, H., Sopala, M., Corrado, C., Bengoudifa, B.-R., Binlich, F., Richardson, P.G., 2014. Panobinostat plus bortezomib and dexamethasone versus placebo plus bortezomib and dexamethasone in patients with relapsed or relapsed and refractory multiple myeloma: a multicentre, randomised, double-blind phase 3 trial. The Lancet. Oncology 15 (11), 1195-1206. 10.1016/S1470-2045(14)70440-1.

Sann, H., Erichsen, J.v., Hessmann, M., Pahl, A., Hoffmeyer, A., 2013. Efficacy of drugs used in the treatment of IBD and combinations thereof in acute DSS-induced colitis in mice. Life sciences 92 (12), 708-718. 10.1016/j.Ifs.2013.01.028. 
Sauer, R., Becker, H., Hohenberger, W., Rodel, C., Wittekind, C., Fietkau, R., Martus, P., Tschmelitsch, J., Hager, E., Hess, C.F., Karstens, J.-H., Liersch, T., Schmidberger, H., Raab, R., 2004. Preoperative versus postoperative chemoradiotherapy for rectal cancer. The New England journal of medicine 351 (17), 1731-1740. 10.1056/NEJMoa040694.

Savage, D.C., 1977. Microbial ecology of the gastrointestinal tract. Annual review of microbiology 31, 107-133. 10.1146/annurev.mi.31.100177.000543.

Schindelin, J., Arganda-Carreras, I., Frise, E., Kaynig, V., Longair, M., Pietzsch, T., Preibisch, S., Rueden, C., Saalfeld, S., Schmid, B., Tinevez, J.-Y., White, D.J., Hartenstein, V., Eliceiri, K., Tomancak, P., Cardona, A., 2012. Fiji: an open-source platform for biologicalimage analysis. Nature methods 9 (7), 676-682. 10.1038/nmeth.2019.

Shahbazi, J., Liu, P.Y., Atmadibrata, B., Bradner, J.E., Marshall, G.M., Lock, R.B., Liu, T., 2016. The Bromodomain Inhibitor JQ1 and the Histone Deacetylase Inhibitor Panobinostat Synergistically Reduce N-Myc Expression and Induce Anticancer Effects. Clinical cancer research : an official journal of the American Association for Cancer Research 22 (10), 2534-2544. 10.1158/1078-0432.CCR-15-1666.

Sheaffer, K.L., Kim, R., Aoki, R., Elliott, E.N., Schug, J., Burger, L., Schubeler, D., Kaestner, $\mathrm{K} . \mathrm{H}$., 2014. DNA methylation is required for the control of stem cell differentiation in the small intestine. Genes \& development 28 (6), 652-664. 10.1101/gad.230318.113.

Shen, S., Wong, C.H., 2016. Bugging inflammation: role of the gut microbiota. Clinical \& translational immunology 5 (4), e72. 10.1038/cti.2016.12.

Shenoy, S., 2016. Genetic risks and familial associations of small bowel carcinoma. World journal of gastrointestinal oncology 8 (6), 509-519. 10.4251/wjgo.v8.i6.509.

Sheth, K.R., Clary, B.M., 2005. Management of hepatic metastases from colorectal cancer. Clinics in colon and rectal surgery 18 (3), 215-223. 10.1055/s-2005-916282.

Shim, J., Karin, M., 2002. The control of mRNA stability in response to extracellular stimuli. Molecules and cells 14 (3), 323-331.

Slotkin, T.A., Seidler, F.J., 1975. Acute and chronic effects of nicotine on synthesis and storage of catecholamines in the rat adrenal medulla. Life sciences 16 (10), 1613-1622.

Smith, D.M., 1977. Health care of people at work-agricultural workers. The Journal of the Society of Occupational Medicine 27 (3), 87-92.

Smith, G., Carey, F.A., Beattie, J., Wilkie, M.J.V., Lightfoot, T.J., Coxhead, J., Garner, R.C., Steele, R.J.C., Wolf, C.R., 2002. Mutations in APC, Kirsten-ras, and p53--alternative genetic pathways to colorectal cancer. Proceedings of the National Academy of Sciences of the United States of America 99 (14), 9433-9438. 10.1073/pnas.122612899.

Smith, R.A., Eschenbach, A.C. von, Wender, R., Levin, B., Byers, T., Rothenberger, D., Brooks, D., Creasman, W., Cohen, C., Runowicz, C., Saslow, D., Cokkinides, V., Eyre, $\mathrm{H}$., 2001. American Cancer Society guidelines for the early detection of cancer: update of early detection guidelines for prostate, colorectal, and endometrial cancers. Also: update 
2001--testing for early lung cancer detection. CA: a cancer journal for clinicians 51 (1), 3875; quiz 77-80.

Solarova, Z., Mojzis, J., Solar, P., 2015. Hsp90 inhibitor as a sensitizer of cancer cells to different therapies (review). International journal of oncology 46 (3), 907-926. 10.3892/ijo.2014.2791.

Sommer, F., Backhed, F., 2013. The gut microbiota--masters of host development and physiology. Nature reviews. Microbiology 11 (4), 227-238. 10.1038/nrmicro2974.

Souza, E.L., Elian, S.D., Paula, L.M., Garcia, C.C., Vieira, A.T., Teixeira, M.M., Arantes, R.M., Nicoli, J.R., Martins, F.S., 2016. Escherichia coli strain Nissle 1917 ameliorates experimental colitis by modulating intestinal permeability, the inflammatory response and clinical signs in a faecal transplantation model. Journal of medical microbiology 65 (3), 201-210. 10.1099/jmm.0.000222.

Sowa, M.E., Bennett, E.J., Gygi, S.P., Harper, J.W., 2009. Defining the human deubiquitinating enzyme interaction landscape. Cell 138 (2), 389-403. 10.1016/j.cell.2009.04.042.

Strahl, B.D., Allis, C.D., 2000. The language of covalent histone modifications. Nature 403 (6765), 41-45. 10.1038/47412.

Subramaniam, R., Mizoguchi, A., Mizoguchi, E., 2016. Mechanistic roles of epithelial and immune cell signaling during the development of colitis-associated cancer. Cancer research frontiers 2 (1), 1-21. 10.17980/2016.1.

Sulciner, D.J., Irani, K., Yu, Z.X., Ferrans, V.J., Goldschmidt-Clermont, P., Finkel, T., 1996. rac1 regulates a cytokine-stimulated, redox-dependent pathway necessary for NF-kappaB activation. Molecular and cellular biology 16 (12), 7115-7121.

Supek, F., Bosnjak, M., Skunca, N., Smuc, T., 2011. REVIGO summarizes and visualizes long lists of gene ontology terms. PloS one 6 (7), e21800. 10.1371/journal.pone.0021800.

Sussman, R.T., Stanek, T.J., Esteso, P., Gearhart, J.D., Knudsen, K.E., McMahon, S.B., 2013. The epigenetic modifier ubiquitin-specific protease 22 (USP22) regulates embryonic stem cell differentiation via transcriptional repression of sex-determining region Y-box 2 (SOX2). The Journal of biological chemistry 288 (33), 24234-24246. 10.1074/jbc.M113.469783.

Swidsinski, A., Ladhoff, A., Pernthaler, A., Swidsinski, S., Loening-Baucke, V., Ortner, M., Weber, J., Hoffmann, U., Schreiber, S., Dietel, M., Lochs, H., 2002. Mucosal flora in inflammatory bowel disease. Gastroenterology 122 (1), 44-54.

Taipale, J., Beachy, P.A., 2001. The Hedgehog and Wnt signalling pathways in cancer. Nature 411 (6835), 349-354. 10.1038/35077219.

Taketo, M.M., Edelmann, W., 2009. Mouse models of colon cancer. Gastroenterology 136 (3), 780-798. 
Tan, K.Y., Tan, S.-M., Tan, A.G.S., Chen, C.Y.Y., Chng, H.-C., Hoe, M.N.Y., 2003. Adult intussusception: experience in Singapore. ANZ journal of surgery 73 (12), 1044-1047.

Tarcic, O., Pateras, I.S., Cooks, T., Shema, E., Kanterman, J., Ashkenazi, H., Boocholez, H., Hubert, A., Rotkopf, R., Baniyash, M., Pikarsky, E., Gorgoulis, V.G., Oren, M., 2016. RNF20 Links Histone H2B Ubiquitylation with Inflammation and Inflammation-Associated Cancer. Cell reports 14 (6), 1462-1476. 10.1016/j.celrep.2016.01.020.

The Medical Letter, I., 2014. A stool DNA test (Cologuard) for colorectal cancer screening. The Medical letter on drugs and therapeutics 56 (1453), 100-101.

Theresa Gorsler, 2013. The role and regulation of histone H2B monoubiquitination during tumorigenesis. PhD Thesis, Göttingen.

Thirunavukarasu, P., Sathaiah, M., Singla, S., Sukumar, S., Karunamurthy, A., Pragatheeshwar, K.D., Lee, K.K.W., Zeh, H.3., Kane, K.M., Bartlett, D.L., 2010. Medullary carcinoma of the large intestine: a population based analysis. International journal of oncology 37 (4), 901-907.

Tian, Z., D'Arcy, P., Wang, X., Ray, A., Tai, Y.-T., Hu, Y., Carrasco, R.D., Richardson, P., Linder, S., Chauhan, D., Anderson, K.C., 2014. A novel small molecule inhibitor of deubiquitylating enzyme USP14 and UCHL5 induces apoptosis in multiple myeloma and overcomes bortezomib resistance. Blood 123 (5), 706-716. 10.1182/blood-2013-05500033.

Tiwari, A.K., Roy, H.K., 2012. Progress against cancer (1971-2011): how far have we come? Journal of internal medicine 271 (4), 392-399. 10.1111/j.1365-2796.2011.02462.x.

Torres, I.O., Fujimori, D.G., 2015. Functional coupling between writers, erasers and readers of histone and DNA methylation. Current opinion in structural biology $35,68-75$. 10.1016/j.sbi.2015.09.007.

Townsend, N., Nichols, M., Scarborough, P., Rayner, M., 2015. Cardiovascular disease in Europe 2015: epidemiological update. European heart journal 36 (40), 2673-2674.

Toyota, M., Ahuja, N., Ohe-Toyota, M., Herman, J.G., Baylin, S.B., Issa, J.P., 1999. CpG island methylator phenotype in colorectal cancer. Proceedings of the National Academy of Sciences of the United States of America 96 (15), 8681-8686.

Turnbaugh, P.J., Ridaura, V.K., Faith, J.J., Rey, F.E., Knight, R., Gordon, J.I., 2009. The effect of diet on the human gut microbiome: a metagenomic analysis in humanized gnotobiotic mice. Science translational medicine 1 (6), 6ra14. 10.1126/scitranslmed.3000322.

Udo, I.A., Abudu, E.K., Uduma, F., 2016. Adult intussusception: An 8 years institutional review. Nigerian medical journal : journal of the Nigeria Medical Association 57 (4), 204207. 10.4103/0300-1652.188324.

Upasana Bedi, 2013. Regulation of H2B monoubiquitination pathway in breast cancer. Ph.D. Thesis, Göttingen. 
van der Flier, Laurens G, Clevers, H., 2009. Stem cells, self-renewal, and differentiation in the intestinal epithelium. Annual review of physiology 71, 241-260.

10.1146/annurev.physiol.010908.163145.

van der Flier, Laurens G, Haegebarth, A., Stange, D.E., van de Wetering, M., Clevers, H., 2009. OLFM4 is a robust marker for stem cells in human intestine and marks a subset of colorectal cancer cells. Gastroenterology 137 (1), 15-17. 10.1053/j.gastro.2009.05.035.

van der Sluis, M., De Koning, Barbara A E, De Bruijn, Adrianus C J M, Velcich, A., Meijerink, J.P.P., van Goudoever, J.B., Buller, H.A., Dekker, J., van Seuningen, I., Renes, I.B., Einerhand, A.W.C., 2006. Muc2-deficient mice spontaneously develop colitis, indicating that MUC2 is critical for colonic protection. Gastroenterology 131 (1), 117-129. 10.1053/j.gastro.2006.04.020.

Vasen, H.F., Watson, P., Mecklin, J.P., Lynch, H.T., 1999. New clinical criteria for hereditary nonpolyposis colorectal cancer (HNPCC, Lynch syndrome) proposed by the International Collaborative group on HNPCC. Gastroenterology 116 (6), 1453-1456.

Veeman, M.T., Slusarski, D.C., Kaykas, A., Louie, S.H., Moon, R.T., 2003. Zebrafish prickle, a modulator of noncanonical Wnt/Fz signaling, regulates gastrulation movements. Current biology : CB 13 (8), 680-685.

Verhulst, J., Ferdinande, L., Demetter, P., Ceelen, W., 2012. Mucinous subtype as prognostic factor in colorectal cancer: a systematic review and meta-analysis. Journal of clinical pathology 65 (5), 381-388. 10.1136/jclinpath-2011-200340.

Vilar, E., Gruber, S.B., 2010. Microsatellite instability in colorectal cancer-the stable evidence. Nature reviews. Clinical oncology 7 (3), 153-162. 10.1038/nrclinonc.2009.237.

Vleugels, J.L.A., van Lanschot, Meta C J, Dekker, E., 2016. Colorectal cancer screening by colonoscopy: putting it into perspective. Digestive endoscopy : official journal of the Japan Gastroenterological Endoscopy Society 28 (3), 250-259. 10.1111/den.12533.

Voskoglou-Nomikos, T., Pater, J.L., Seymour, L., 2003. Clinical predictive value of the in vitro cell line, human xenograft, and mouse allograft preclinical cancer models. Clinical cancer research : an official journal of the American Association for Cancer Research 9 (11), 4227-4239.

Wang, E., Kawaoka, S., Yu, M., Shi, J., Ni, T., Yang, W., Zhu, J., Roeder, R.G., Vakoc, C.R., 2013. Histone H2B ubiquitin ligase RNF20 is required for MLL-rearranged leukemia. Proceedings of the National Academy of Sciences of the United States of America 110 (10), 3901-3906. 10.1073/pnas.1301045110.

Wang, Y., Duan, H., Yang, H., Lin, J., 2015. A pooled analysis of alcohol intake and colorectal cancer. International journal of clinical and experimental medicine 8 (5), 68786889.

Weber, A., Heinlein, M., Dengjel, J., Alber, C., Singh, P.K., Hacker, G., 2016. The deubiquitinase Usp27x stabilizes the BH3-only protein Bim and enhances apoptosis. EMBO reports 17 (5), 724-738. 10.15252/embr.201541392. 
White, M.C., Holman, D.M., Boehm, J.E., Peipins, L.A., Grossman, M., Henley, S.J., 2014. Age and cancer risk: a potentially modifiable relationship. American journal of preventive medicine 46 (3 Suppl 1), S7-15. 10.1016/j.amepre.2013.10.029.

Whitesell, L., Lindquist, S.L., 2005. HSP90 and the chaperoning of cancer. Nature reviews. Cancer 5 (10), 761-772. 10.1038/nrc1716.

Wicha, M.S., Liu, S., Dontu, G., 2006. Cancer stem cells: an old idea--a paradigm shift. Cancer research 66 (4), 1883-90; discussion 1895-6. 10.1158/0008-5472.CAN-05-3153.

Wu, N., Yang, X., Zhang, R., Li, J., Xiao, X., Hu, Y., Chen, Y., Yang, F., Lu, N., Wang, Z., Luan, C., Liu, Y., Wang, B., Xiang, C., Wang, Y., Zhao, F., Gao, G.F., Wang, S., Li, L., Zhang, H., Zhu, B., 2013. Dysbiosis signature of fecal microbiota in colorectal cancer patients. Microbial ecology 66 (2), 462-470. 10.1007/s00248-013-0245-9.

Xavier, R.J., Podolsky, D.K., 2007. Unravelling the pathogenesis of inflammatory bowel disease. Nature 448 (7152), 427-434. 10.1038/nature06005.

Xiao, H., Tian, Y., Yang, Y., Hu, F., Xie, X., Mei, J., Ding, F., 2015. USP22 acts as an oncogene by regulating the stability of cyclooxygenase-2 in non-small cell lung cancer. Biochemical and biophysical research communications 460 (3), 703-708. 10.1016/j.bbrc.2015.03.093.

Xu, H., Liu, Y.-L., Yang, Y.-M., Dong, X.-S., 2012. Knock-down of ubiquitin-specific protease 22 by micro-RNA interference inhibits colorectal cancer growth. International journal of colorectal disease 27 (1), 21-30. 10.1007/s00384-011-1275-8.

Yang, D.-d., Cui, B.-B., Sun, L.-y., Zheng, H.-q., Huang, Q., Tong, J.-X., Zhang, Q.-F., 2011. The co-expression of USP22 and BMI-1 may promote cancer progression and predict therapy failure in gastric carcinoma. Cell biochemistry and biophysics 61 (3), 703-710. 10.1007/s12013-011-9229-x.

Yatsunenko, T., Rey, F.E., Manary, M.J., Trehan, I., Dominguez-Bello, M.G., Contreras, M., Magris, M., Hidalgo, G., Baldassano, R.N., Anokhin, A.P., Heath, A.C., Warner, B., Reeder, J., Kuczynski, J., Caporaso, J.G., Lozupone, C.A., Lauber, C., Clemente, J.C., Knights, D., Knight, R., Gordon, J.I., 2012. Human gut microbiome viewed across age and geography. Nature 486 (7402), 222-227. 10.1038/nature11053.

Ye, J., Coulouris, G., Zaretskaya, I., Cutcutache, I., Rozen, S., Madden, T.L., 2012. PrimerBLAST: a tool to design target-specific primers for polymerase chain reaction. BMC bioinformatics 13, 134. 10.1186/1471-2105-13-134.

Yoshida, K., Miki, Y., 2004. Role of BRCA1 and BRCA2 as regulators of DNA repair, transcription, and cell cycle in response to DNA damage. Cancer science 95 (11), 866871.

Young, B., Wheater, P.R., 2007. Wheater's functional histology: A text and colour atlas ; [online access + interactive extras studentconsult.com], 5th ed. Churchill Livingstone Elsevier, Philadelphia, Pa., 437 pp. 
Young, G.P., Symonds, E.L., Allison, J.E., Cole, S.R., Fraser, C.G., Halloran, S.P., Kuipers, E.J., Seaman, H.E., 2015. Advances in Fecal Occult Blood Tests: the FIT revolution. Digestive diseases and sciences 60 (3), 609-622. 10.1007/s10620-014-3445-3.

Yu, X.-F., Zou, J., Dong, J., 2014. Fish consumption and risk of gastrointestinal cancers: a meta-analysis of cohort studies. World journal of gastroenterology 20 (41), 15398-15412. 10.3748/wjg.v20.i41.15398.

Zeissig, S., Blumberg, R.S., 2013. Commensal microbiota and NKT cells in the control of inflammatory diseases at mucosal surfaces. Current opinion in immunology 25 (6), 690696. 10.1016/j.coi.2013.09.012.

Zeissig, S., Kaser, A., Dougan, S.K., Nieuwenhuis, E.E.S., Blumberg, R.S., 2007. Role of NKT cells in the digestive system. III. Role of NKT cells in intestinal immunity. American journal of physiology. Gastrointestinal and liver physiology 293 (6), G1101-5. 10.1152/ajpgi.00342.2007.

Zhang, F., Yu, X., 2011. WAC, a functional partner of RNF20/40, regulates histone H2B ubiquitination and gene transcription. Molecular cell 41 (4), 384-397. 10.1016/j.molcel.2011.01.024.

Zhang, P.-Y., Yang, Y.-J., Xue, Y., Fu, J., Zhang, C.-X., Wang, Y., Yang, Y., Shi, H., 2015. Cancer stem cells: targeting tumors at the source. European review for medical and pharmacological sciences 19 (10), 1821-1828.

Zhang, X.-Y., Pfeiffer, H.K., Thorne, A.W., McMahon, S.B., 2008a. USP22, an hSAGA subunit and potential cancer stem cell marker, reverses the polycomb-catalyzed ubiquitylation of histone H2A. Cell cycle (Georgetown, Tex.) 7 (11), 1522-1524. 10.4161/cc.7.11.5962.

Zhang, X.-Y., Varthi, M., Sykes, S.M., Phillips, C., Warzecha, C., Zhu, W., Wyce, A., Thorne, A.W., Berger, S.L., McMahon, S.B., 2008b. The putative cancer stem cell marker USP22 is a subunit of the human SAGA complex required for activated transcription and cellcycle progression. Molecular cell 29 (1), 102-111. 10.1016/j.molcel.2007.12.015.

Zhang, Z.-J., Zheng, Z.-J., Kan, H., Song, Y., Cui, W., Zhao, G., Kip, K.E., 2011. Reduced risk of colorectal cancer with metformin therapy in patients with type 2 diabetes: a metaanalysis. Diabetes care 34 (10), 2323-2328. 10.2337/dc11-0512.

Zhao, Y., Huang, Z.-J., Rahman, M., Luo, Q., Thorlacius, H., 2013. Radicicol, an Hsp90 inhibitor, inhibits intestinal inflammation and leakage in abdominal sepsis. The Journal of surgical research 182 (2), 312-318. 10.1016/j.jss.2012.10.038.

Zhou, X., Fan, L.X., Peters, D.J.M., Trudel, M., Bradner, J.E., Li, X., 2015. Therapeutic targeting of BET bromodomain protein, Brd4, delays cyst growth in ADPKD. Human molecular genetics 24 (14), 3982-3993. 10.1093/hmg/ddv136.

Zhu, B., Zheng, Y., Pham, A.-D., Mandal, S.S., Erdjument-Bromage, H., Tempst, P., Reinberg, D., 2005. Monoubiquitination of human histone H2B: the factors involved and their roles in HOX gene regulation. Molecular cell 20 (4), 601-611. 10.1016/j.molcel.2005.09.025. 


\section{ACKNOWLEDGEMENTS}

There are many people who have supported me in their own special way over the past four years. Therefore, I would like to take the opportunity to express my appreciation.

I would like to show my greatest gratitude to Prof. Steven A. Johnsen who took over the supervision of this project and welcomed me to his wonderful group. Your motivation, encouragement and suggestions are invaluable and you helped me experience the exciting and quickly evolving field of science on another level. Your continuous support has encouraged me to believe in my own scientific ideas and skills.

I am very grateful to Dr. Yvonne Begus-Nahrmann and Prof. Dr. Matthias Dobbelstein for their help during the early phases of this project. My fascination for cancer research has grown under your guidance. Thank you for all the scientific discussions and the support.

Moreover, I sincerely thank my thesis committee members, Prof. Dr. Heidi Hahn and Prof. Dr. Holger Reichardt for their helpful suggestions and constructive discussions as well as for their genuine interest in my project.

Special thanks to AG Johnsen for your companionship. Immediately after joining the group, your openness, kindness and humor made it really easy for me to fit in. On a special note, the advice and comments given by Florian have been a great help during the course of this project.

Moreover, I would like to thank Dominik, Feda, Florian, and Madhobi for proofreading this manuscript. 
In addition, I am grateful for the assistance given by all the students I supervised and who showed me how much I enjoy passing on my knowledge and scientific tricks. Thank you for all the fun moments we have shared, Arshiya, Elina, Franziska, Frederike, Ivan, Lorenz, Madhobi, Mahmoud, Melanie, and Pia.

Finally, I cannot find words to express my gratitude to my loved ones. I am so lucky to have you in my life. The constant encouragement, understanding and unconditional love of my parents and my brother have made me who I am today. Thank you, Dominik, for your continuous support and finding the right words. You were always there for me when I needed a shoulder to lean on. For everything else that I failed to mention, thank you! 